\title{
NIST CENTER FOR NEUTRON RESEARCH
}
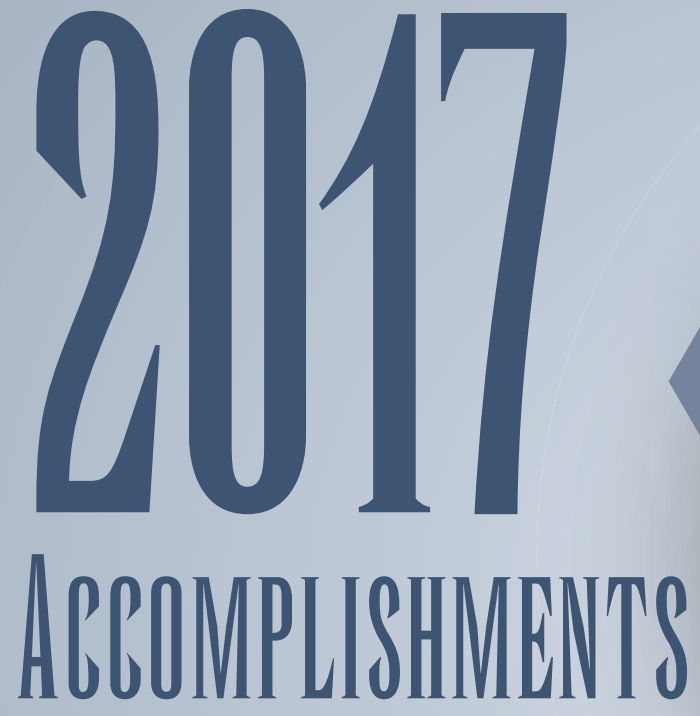

AND OPPORTUNITILS

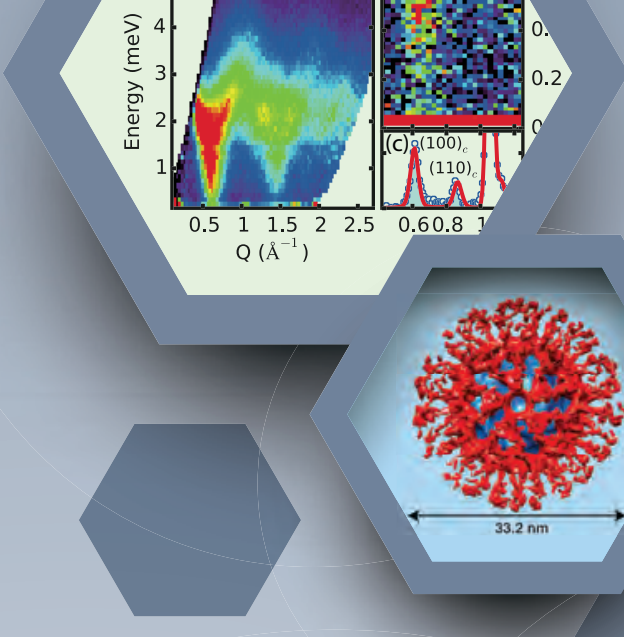




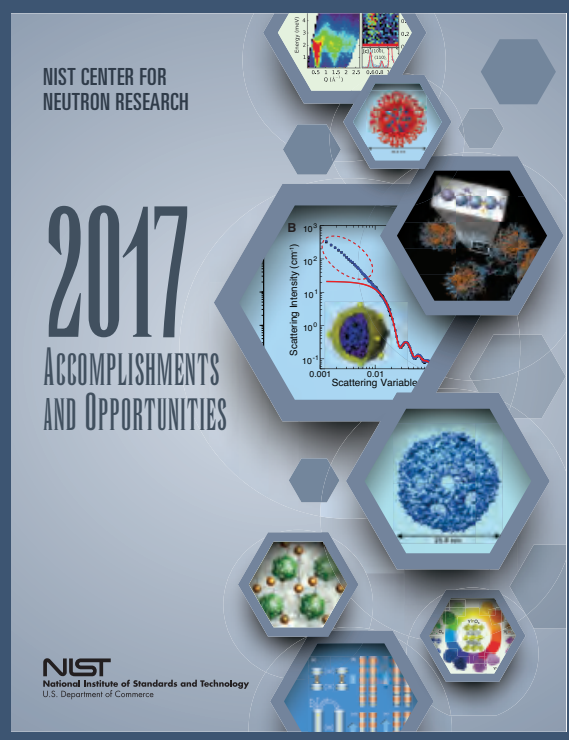

\section{ON THE COVER}

The NCNR's neutron

source provides a valuable research tool for a wide variety of scientific fields from biological systems, engineering materials, to magnetic structures, and more. The cover features data from only a few of the experiments highlighted in this report. 


\title{
2017 NIST Center for Neutron Research Accomplishments and Opportunities
}

\author{
Robert M. Dimeo, Director
}

Steven R. Kline, Editor

This publication is available free of charge from:

https://doi.org/10.6028/NIST.SP.1216

December 2017

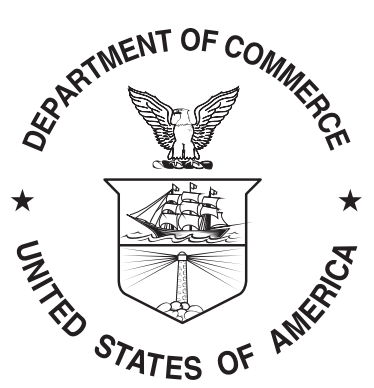

U.S. Department of Commerce Wilbur L. Ross, Jr., Secretary

National Institute of Standards and Technology Walter Copan, NIST Director and Undersecretary of

Commerce for Standards and Technology 


\section{DISCLAIMER}

Certain commercial entities, equipment, or materials may be identified in this document in order to describe an experimental procedure or concept adequately. Such identification is not intended to imply recommendation or endorsement by the National Institute of Standards and Technology, nor is it intended to imply that the entities, materials, or equipment are necessarily the best available for the purpose. Error bars in figures and uncertainties in the text represent one standard deviation unless otherwise stated.

National Institute of Standards and Technology Special Publication 1216, 96 pages

(December 2017)

CODEN: NSPUE2

This publication is available free of charge from: https://doi.org/10.6028/NIST.SP.1216 


\section{Table of Contents}

\section{ii FOREWORD}

1 THE NIST CENTER FOR NEUTRON RESEARCH

2 NIST CENTER FOR NEUTRON RESEARCH INSTRUMENTS

\section{NCNR IMAGES 2017}

\section{HIGHLIGHTS}

\section{BIOLOGY}

6 Determination of functional collective motions in a protein at atomic resolution using coherent neutron scattering,

L. Hong, et al. (CHRNS)

8 Using small-angle neutron scattering to characterize the NISTmAb reference material, M. M. Castellanos, et al. (CHRNS)

\section{CHEMICAL PHYSICS}

10 From serendipity to rational design: tuning the blue trigonal bipyramidal $\mathrm{Mn}^{3+}$ chromophore to violet and purple through application of chemical pressure, J. Li, et al.

12 Capture and release of chlorine and bromine gas with a hybrid sponge, Y. Tulchinsky, et al.

14 Stabilizing superionic-conducting structures at subambient temperatures via mixed-anion solid solutions of monocarbacloso-polyborate salts, W. S. Tang, et al. (CHRNS)

16 Discovery of a dynamic odd-even effect in liquid n-alkanes near melting points, K. Yang, et al.

18 New metastable materials help answer what makes iron so special for superconductivity, X. Zhou, et al.

\section{CONDENSED MATTER}

20 Long-lived Higgs amplitude mode in a two-dimensional quantum antiferromagnet near the quantum critical point, T. Hong, et al. (CHRNS)

22 Complex three-dimensional magnetic ordering in segmented nanowire arrays, A. J. Grutter, et al. (CHRNS)

24 Enhancing magnetic order in topological insulators with antiferromagnets, Q. L. He, et al.

26 Atomic scale engineering of multiferroicity in $\mathrm{LuFeO}_{3} / \mathrm{LuFe}_{2} \mathrm{O}_{4}$ superlattices, J. A. Mundy, et al.

28 Nascent magnetic order and quantum fluctuations in a spin orbital liquid, K. W. Plumb, et al. (CHRNS)

30 Orbital-exchange and fractional quantum excitations in an f-electron metal $\mathrm{Yb}_{2} \mathrm{Pt}_{2} \mathrm{~Pb}$, L. S. Wu, et al.

\section{ENGINEERING PHYSICS}

32 Overview of residual stresses in additive manufactured parts, J. Milner, et al.

34 Stresses in automotive fastener joints, T. Gnäupel-Herold, et al.

\section{NEUTRON PHYSICS}

36 Holography with a neutron interferometer, D. Sarenac, et al.

38 aCORN: A precision measurement of the electronantineutrino angular correlation in free neutron beta decay, M. T. Hassan, et al.

\section{SOFT MATTER}

40 Conformational studies of virus/polymer conjugates and their applications in medicine, P. W. Lee, et al. (CHRNS)

42 Tuning the morphology of complex oil-in-water-in-oil nanoemulsions using frustrated spontaneous curvature in surfactant mixtures, M. Zhang, et al. (CHRNS)

44 Small nanoparticles disentangle polymer chains in nanocomposites, E. Senses, et al. (CHRNS)

46 Measuring the interaction of solvated polymers and surfaces, R. J. Sheridan, et al. (nSOFT)

48 Understanding the recovery of tough, nanostructured hydrogels after deformation, C. G. Wiener, et al. (CHRNS)

50 Fingerprinting molecular deformation of polymers, Y. Wang, et al. (CHRNS)

\section{ADVANCES IN MEASUREMENT}

52 Peering inside fuel cell electrodes, D. S. Hussey, et al.

53 Monte Carlo simulation algorithm for B-DNA, S. C. Howell, et al.

54 Improved sample characterization with simultaneous neutron and X-ray tomography, J. M. LaManna, et al.

55 NEUTRON SOURCE OPERATIONS

58 FACILITY DEVELOPMENT

62 SERVING THE SCIENCE AND TECHNOLOGY COMMUNITY

65 THE CENTER FOR HIGH RESOLUTION NEUTRON SCATTERING (CHRNS)

692017 AWARDS

71 PUBLICATIONS: AUGUST 1, 2016 TO JULY 31, 2017

90 INSTRUMENTS AND CONTACTS

92 NIST CENTER FOR NEUTRON RESEARCH CONTACTS 


\section{Foreword}

$\mathrm{t}$ is with great pleasure that I present to you this year's annual report for the NIST Center for Neutron Research, containing a selection of the NCNR's accomplishments and opportunities for 2017. It was a great year for facility operations: in CY 2017 the reactor operated at full power for 228 days with $98 \%$ reliability. Both cold sources operated with very good reliability.

This year was marked by significant developments of two new neutron scattering instruments soon to be available to users. The vSANS vessel arrived on three flatbed trucks in March and they were installed on the NG-3 beamline in April. After an intense period of installation, first neutrons were detected on August 11th and the first science measurement was performed soon thereafter. This new capability will offer unprecedented capabilities to the scientific community and we are excited to make it available for proposals and beamtime requests in 2018. The CANDOR instrument also achieved a milestone when the prototype scintillation detector achieved a neutron detection efficiency comparable to ${ }^{3} \mathrm{He}$ gas-based detectors, a feat that surpassed all performance expectations for the instrument. We are all looking forward to reaching the milestone of first detected neutrons in CANDOR in 2018. In addition to instrument developments, a new laboratory in the north end of the guide hall extension designed for handling activated samples was completed this year and the lab is now open to users.

There were numerous developments designed to improve facility performance, reliability, and extend the lifetime of the reactor to ensure that the NCNR provides neutron measurements to the scientific community far into the future. As of this writing, the reactor is amid an extended outage in which the reactor shim arms are being replaced, the primary pump system is being upgraded, the reactor control console is undergoing

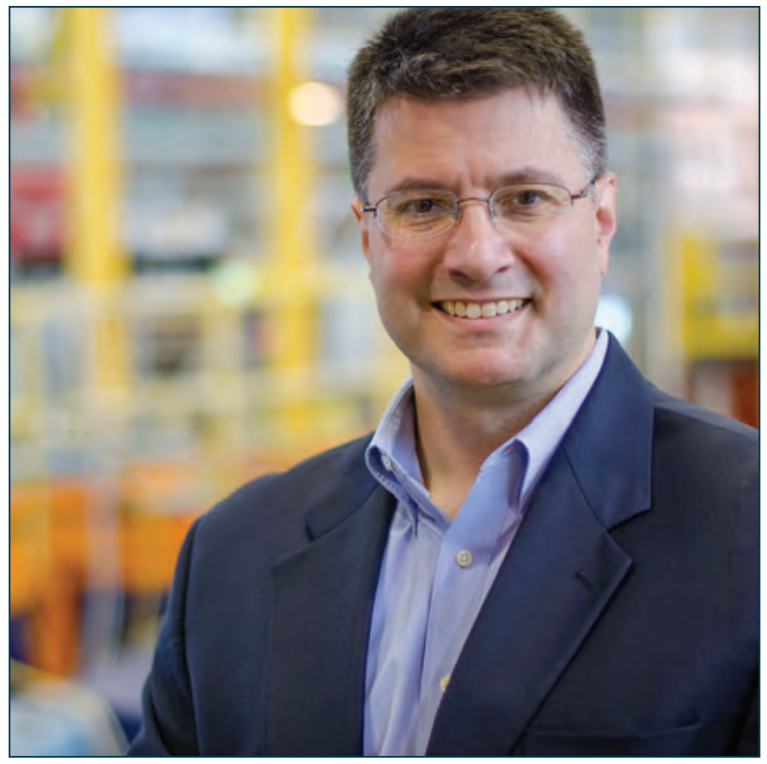

an upgrade, and a new higher capacity refrigerator is being commissioned for use with the existing cold sources. In addition, improvements are being made at MACS and the BT-7 TAS instrument to improve operational reliability. Each one of these items are major projects and the focus of the staff is exclusively focused on completing these tasks successfully so that we may restart scientific operations in January. I encourage you to read more about these projects in this report.

As always, the reason for the NCNR is to provide the scientific community with the best tools to perform research using neutrons. Over the last year the community produced an outstanding body of research from measurements performed here. I am excited to share with you a selection of these highlights from our users. I hope that you enjoy reading through this report as much as I did.

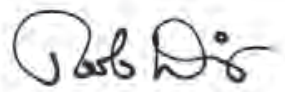




\section{The NIST Center for Neutron Research}

$\mathrm{N}$ eutrons provide a uniquely effective probe of the structure and dynamics of materials ranging from water moving near the surface of proteins to magnetic domains in memory storage materials. The properties of neutrons (outlined below) can be exploited using a variety of measurement techniques to provide information not otherwise available. The positions of atomic nuclei in crystals, especially of those of light atoms, can be determined precisely. Atomic motion can be directly measured and monitored as a function of temperature or pressure. Neutrons are especially sensitive to hydrogen, so that hydrogen motion can be followed in $\mathrm{H}$-storage materials and water flow in fuel cells can be imaged. Residual stresses such as those deep within oil pipelines or in highway trusses can be mapped. Neutron-based measurements contribute to a broad spectrum of activities including engineering, materials development, polymer dynamics, chemical technology, medicine, and physics.

The NCNR's neutron source provides the intense, conditioned beams of neutrons required for these types of measurements. In addition to the thermal neutron beams from the heavy water moderator, the NCNR has two liquid hydrogen moderators, or cold sources which supply neutrons to three-fourths of the instruments. One is a large area moderator and the other is smaller, but with high brightness. These moderators provide long wavelength guided neutron beams for industrial, government, and academic researchers.

There are currently 29 experiment stations: 12 are used for neutron physics, analytical chemistry, or imaging, and 17 are beam facilities for neutron scattering research. The subsequent pages provide a schematic description of our instruments. More complete descriptions can be found at www.ncnr.nist.gov/instruments/. The second guide hall is currently populated with seven instruments. Two new cold neutron instruments are under development, including a very small angle neutron scattering instrument (VSANS), and a quasi-white beam neutron reflectometer (CANDOR).

The Center supports important NIST measurement needs, but is also operated as a major national user facility with merit-based access made available to the entire U.S. technological community. Each year, about 2000 research participants from government, industry, and academia from all areas of the country are served by the facility (see pp. 62). Beam time for research to be published in the open literature is without cost to the user, but full operating costs are recovered for proprietary research. Access is gained mainly through a web-based, peer-reviewed proposal system with user time allotted by a beamtime allocation committee twice a year. For details see www.ncnr.nist.gov/beamtime.html. The National Science Foundation and NIST co-fund the Center for High Resolution Neutron Scattering (CHRNS) that currently operates five of the world's most advanced instruments (see pp. 65). Time on CHRNS instruments is made available through the proposal system. Some access to beam time for collaborative measurements with the NIST science staff can also be arranged on other instruments.

\section{Why Neutrons?}

Neutrons reveal properties not readily probed by photons or electrons. They are electrically neutral and therefore easily penetrate ordinary matter. They behave like microscopic magnets, propagate as waves, can set particles into motion, losing or gaining energy and momentum in the process, and they can be absorbed with subsequent emission of radiation to uniquely fingerprint chemical elements.

WAVELENGTHS - in practice range from $\approx 0.01 \mathrm{~nm}$ (thermal) to $\approx 1.5 \mathrm{~nm}$ (cold) $(1 \mathrm{~nm}=10 \AA)$ ), allowing the formation of observable interference patterns when scattered from structures as small as atoms to as large as biological cells.

ENERGIES - of millielectronvolts, the same magnitude as atomic motions. Exchanges of energy as small as nanoelectronvolts and as large as tenths of electronvolts can be detected between samples and neutrons, allowing motions in folding proteins, melting glasses and diffusing hydrogen to be measured.

SELECTIVITY - in scattering power varies from nucleus to nucleus somewhat randomly. Specific isotopes can stand out from other isotopes of the same kind of atom. Specific light atoms, difficult to observe with $\mathrm{X}$-rays, are revealed by neutrons. Hydrogen, especially, can be distinguished from chemically equivalent deuterium, allowing a variety of powerful contrast techniques.

MAGNETISM - makes the neutron sensitive to the magnetic moments of both nuclei and electrons, allowing the structure and behavior of ordinary and exotic magnetic materials to be detailed precisely.

NEUTRALITY - of the uncharged neutrons allows them to penetrate deeply without destroying samples, passing through walls that condition a sample's environment, permitting measurements under extreme conditions of temperature and pressure.

CAPTURE - characteristic radiation emanating from specific nuclei capturing incident neutrons can be used to identify and quantify minute amounts of elements in samples as diverse as ancient pottery shards and lake water pollutants. 
[3]

3]

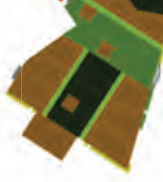

[2]

[1] BT-5 Perfect Crystal Ultra-Small Angle Neutron Scattering (USANS) Diffractometer for microstructure up to $10^{4} \mathrm{~nm}$. GHRNS.

[2] BT-4 Filter Analyzer Neutron Spectrometer with cooled Be/Graphite filter analyzer for chemical spectroscopy and thermal triple axis spectrometer.

[3] BT-2 Neutron Imaging Facility for imaging hydrogenous matter in large components such as water in fuel cells and lubricants in engines, in partnership with General Motors and DOE.

[4] BT-1 Powder Diffractometer with 32 detectors; incident wavelengths of $0.208 \mathrm{~nm}, 0.154 \mathrm{~nm}$, and $0.159 \mathrm{~nm}$, with resolution up to $\Delta \mathrm{d} / \mathrm{d} \cong 8 \times 10^{-4}$.

[5] BT-9 Multi Axis Crystal Spectrometer (MACS II), a cold neutron spectrometer for ultra high sensitivity access to dynamic correlations in condensed matter on length scales from $0.1 \mathrm{~nm}$ to $50 \mathrm{~nm}$ and energy scales from $2.2 \mathrm{meV}$ to $20 \mathrm{meV}$. GHRNS.
[9]

]

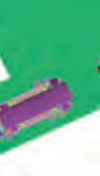

\section{[1]}

[29]

[15]

[13]

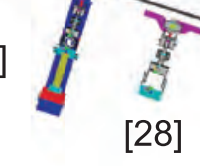

[28]

[27]

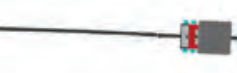

[16]

[6] BT-8 Residual Stress Diffractometer optimized for depth profiling of residual stresses in large components.

[7] BT-7 Thermal Triple Axis Spectrometer with large double focusing monochromator and interchangeable analyzer/detectors systems.

[8] VT-5 Thermal Neutron Capture Prompt Gamma-ray Activation Analysis Instrument used for quantitative elemental analysis of bulk materials

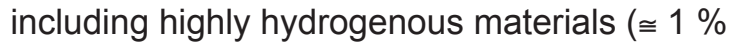
$\mathrm{H})$ such as foods, oils, and biological materials.

[9] NG-A Neutron Spin-Echo Spectrometer (NSE) for measuring dynamics from $5 \mathrm{ps}$ to $100 \mathrm{~ns}$.

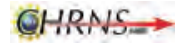

[10] NG-B 10 m SANS for macromolecular structure measurements. $n \mathrm{Soft}$ 


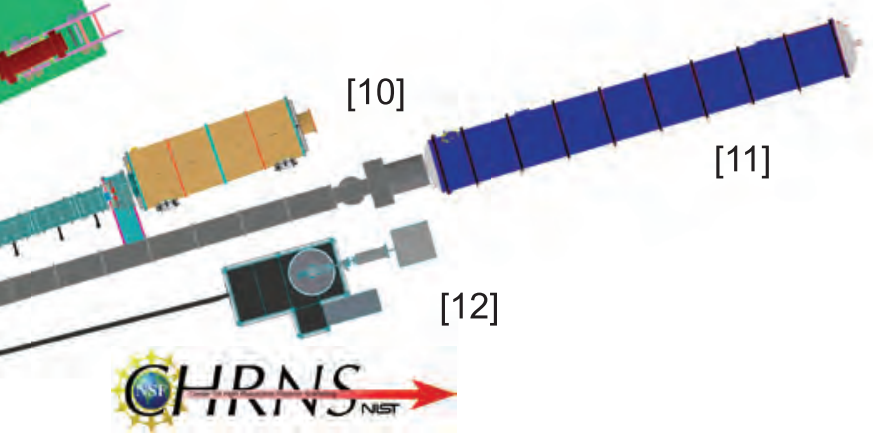

The Center for High Resolution Neutron Scattering (CHRNS) is a partnership between NIST and the National Science Foundation that develops and operates neutron scattering instrumentation for use by the scientific community. The following instruments are part of the Center: 1 (USANS), 5 (MACS II), 9 (NSE), 11 (NG-B 30m SANS), 16 (HFBS), and 17 (vSANS).

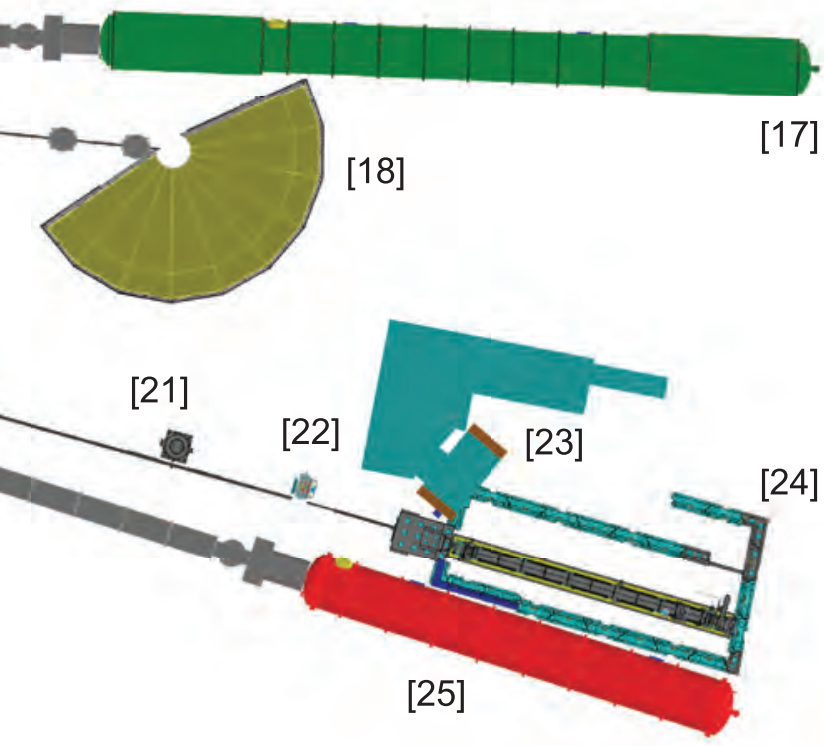

[11] NG-B 30 m SANS for microstructure measurements. QHRNS

[12] NG-C Neutron lifetime experiment.

[13] NG-D Cold neutron capture Prompt Gamma Activation Analysis, for quantitative elemental analysis of bulk materials.

[14] NG-D MAGIK off-specular reflectometer for studies of thin-film samples with in-plane structure.

[15] NG-D Polarized Beam Reflectometer (PBR) for measuring reflectivities as low as $10^{-8}$ to determine subsurface structure.
[16] NG-2 Backscattering Spectrometer (HFBS) high intensity inelastic scattering instrument with energy resolution $<1 \mu \mathrm{eV}$, for studies of motion in molecular and biological systems.

[17] NG-3 vSANS Single measurement investigation of lengths from $1 \mathrm{~nm}$ to 2 micron. @HRAvS $\longrightarrow$

[18] NG-4 Disk Chopper Time-of-Flight Spectrometer for diffusive motions and low energy dynamics. Wavelengths from $\cong 0.18 \mathrm{~nm}$ to $2.0 \mathrm{~nm}$ and energy resolutions from $\cong 2 \mathrm{meV}$ to $<10 \mathrm{meV}$.

[19] NG-5 Spin-Polarized Triple Axis Spectrometer (SPINS) using cold neutrons with position sensitive detector capability for high-resolution studies.

[20] NG-5 Cold Neutron Depth Profiling for profiling of subsurface elemental composition.

[21] NG-6 Precision measurement of the magnetic dipole moment of the neutron.

[22] NG-6 Precision measurement of neutron flux.

[23] NG-6 LAND detector development, neutron source calibration, and neutron cross section measurement.

[24] NG-6 Cold Neutron Imaging Facility for imaging hydrogenous matter in large components such as water in fuel cells and lubricants in engines.

[25] NG-7 30 m SANS for microstructure measurements, in partnership with ExxonMobil and University of Minnesota's IPrime.

[26] NG-7 PHADES Cold neutron test station.

[27] NG-7 Neutron Interferometry and Optics Station with perfect crystal silicon interferometer. A vibration isolation system provides exceptional phase stability and fringe visibility.

[28] NG-7 Neutron Physics Interferometry Test Bed for quantum information science.

[29] NG-7 Horizontal Sample Reflectometer allows reflectivity measurements of free surfaces, liquid/vapor interfaces, as well as polymer coatings. 


\section{NCNR Images 2017}

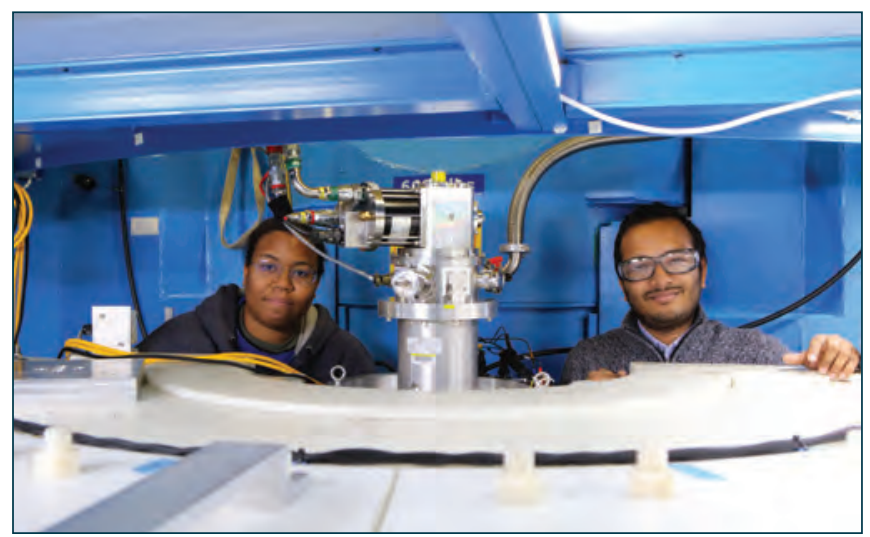

Shannon Watson and Taufique Hassan of the NCNR readying the polarized helium sample environment at MACS.

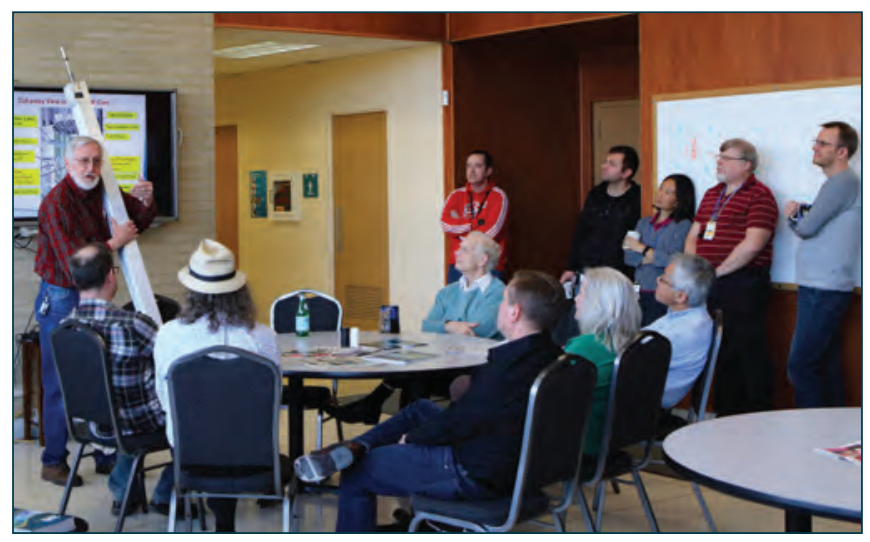

The NCNR's Bob Williams gives a "Coffee and Neutrons" talk about the reactor as the NCNR approaches its 50th anniversary.

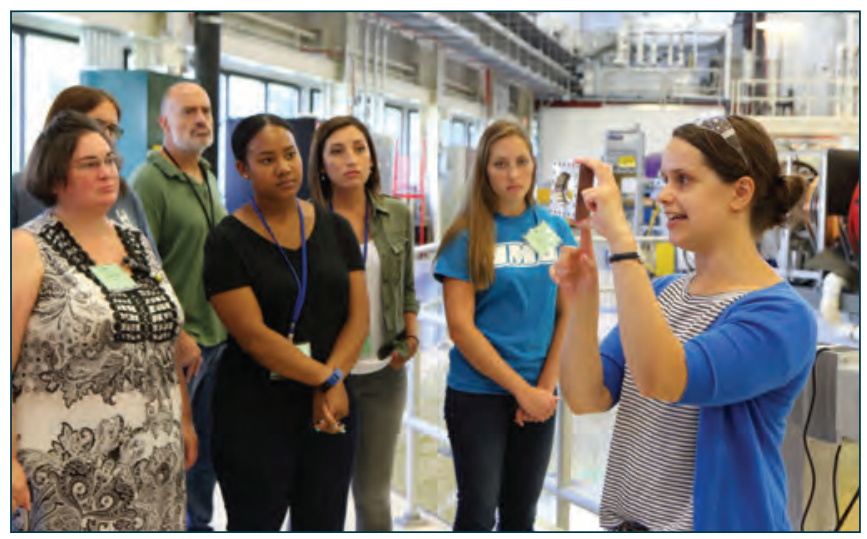

Teachers from the NIST Summer Institute for Middle School Science Teachers are on a field trip, learning how neutron spin-echo is used in soft matter research from the NCNR's Liz Kelley.

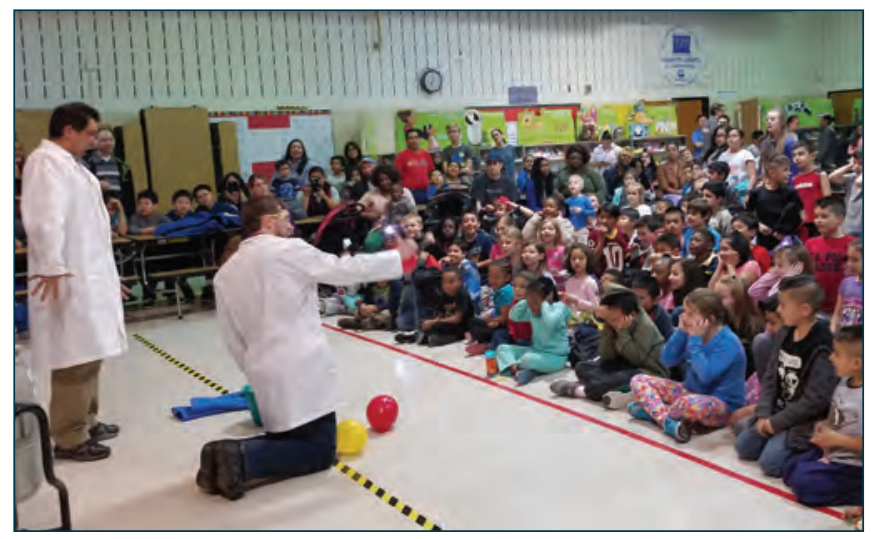

Juscelino Leão and Jeff Krzywon of the NCNR visit Gaithersburg Elementary school and scare the children.

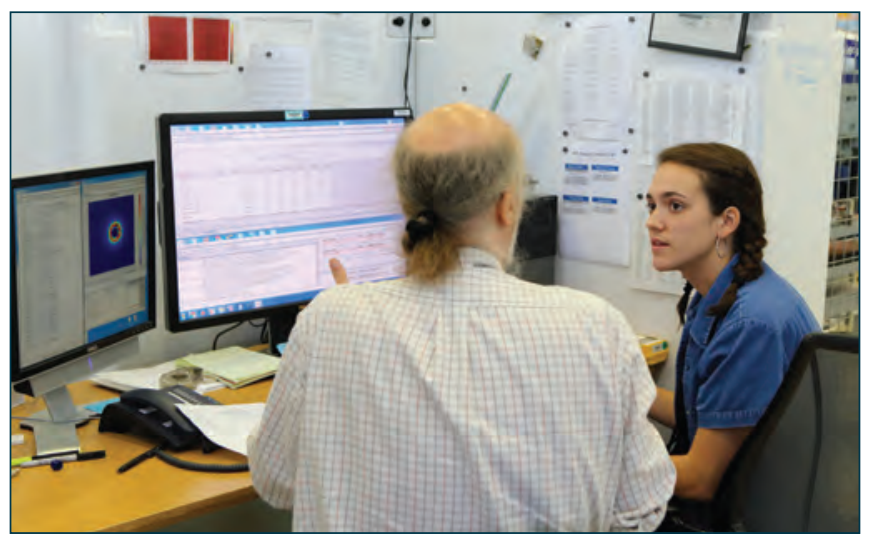

Paul Butler of the NCNR and SURF student Emily Blick discuss their measurements of lipid cubic phases at the NG-B 10m SANS.

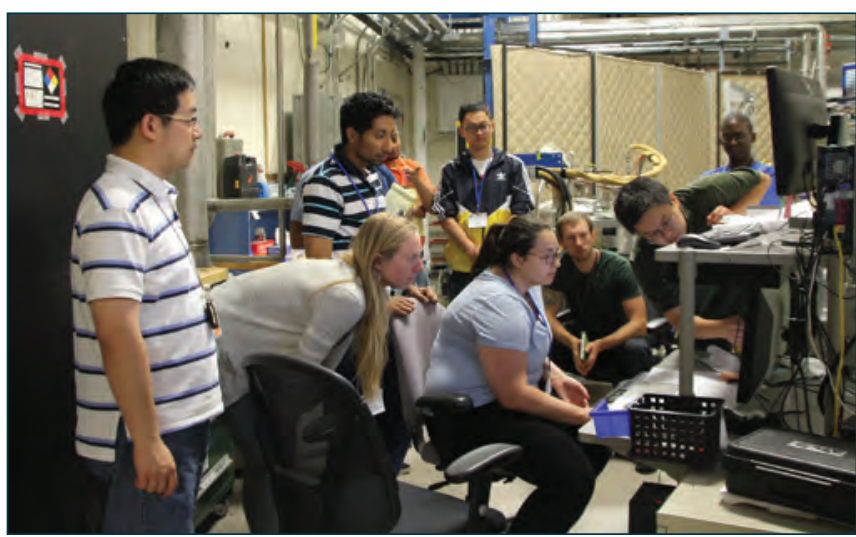

Yang Zhou and Zhijun Xu of the NCNR instruct students how to set up their measurement of colossal magnetoresistive spin dynamics at BT7 during the 2017 Summer School. 


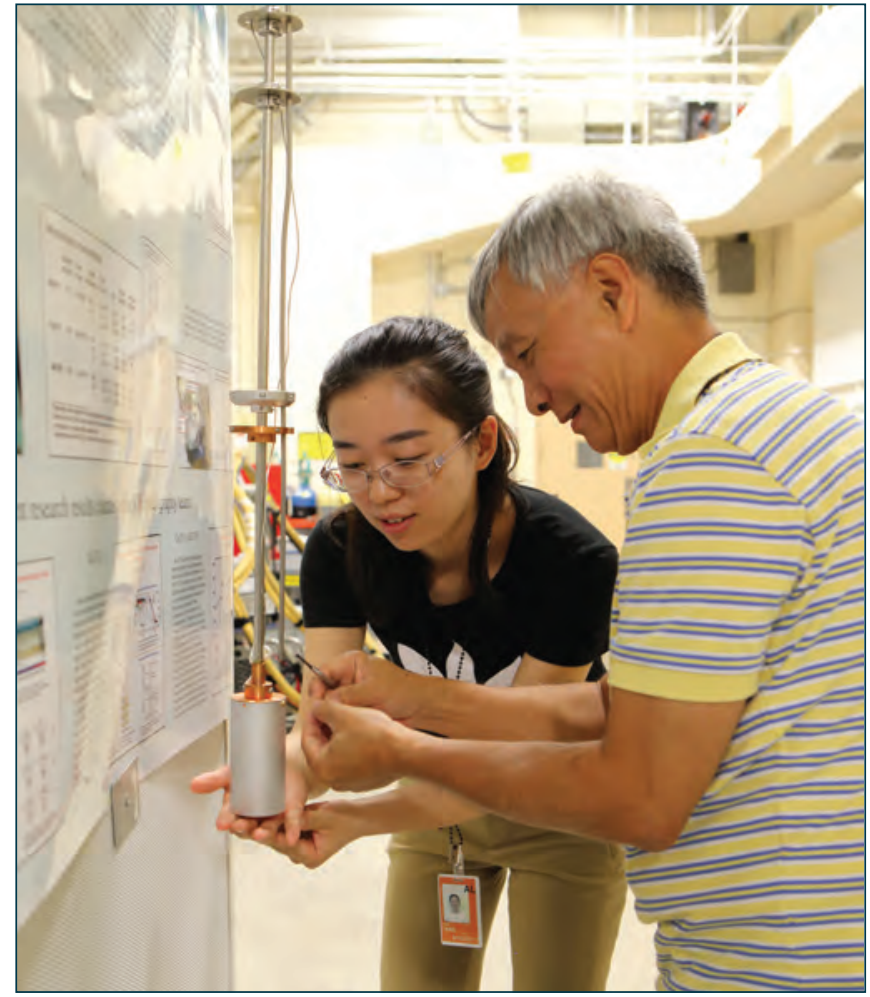

Ying Tang (Guilin University of Technology, China) and Qingzhen Huang (NCNR) prepare a sample for BT1.

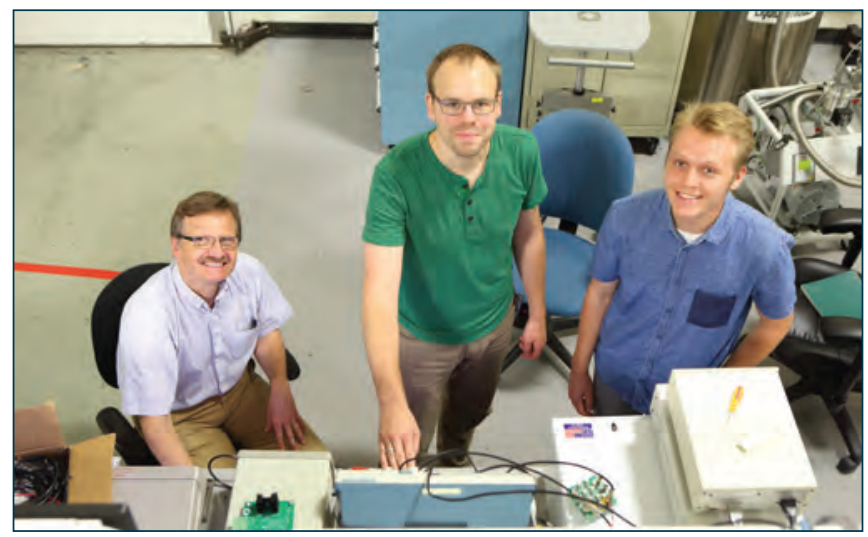

Collin Broholm, Jonas Kindervater, and Tim Reeder (Johns Hopkins University) at BT9 MACS studying the time-dependent dynamics of a spin ice material

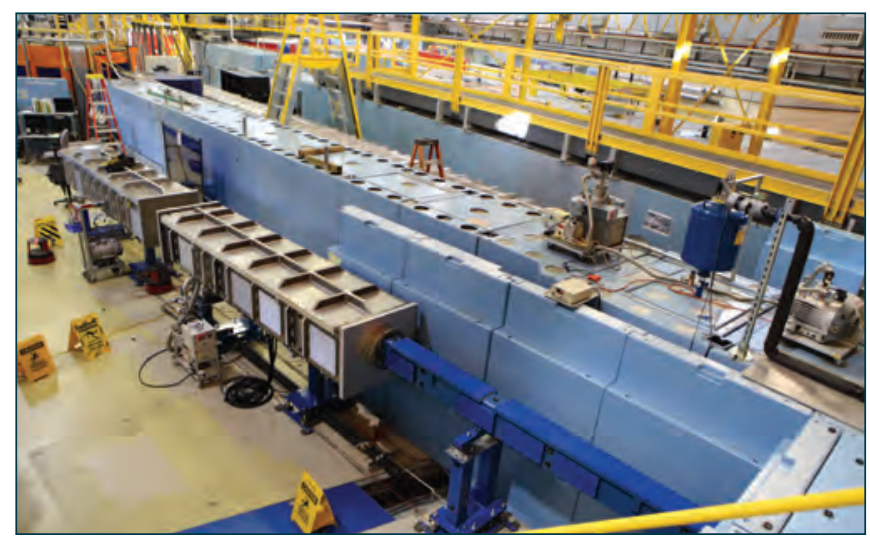

The installation and alignment of the pre-sample flight path for CANDOR is nearing completion.

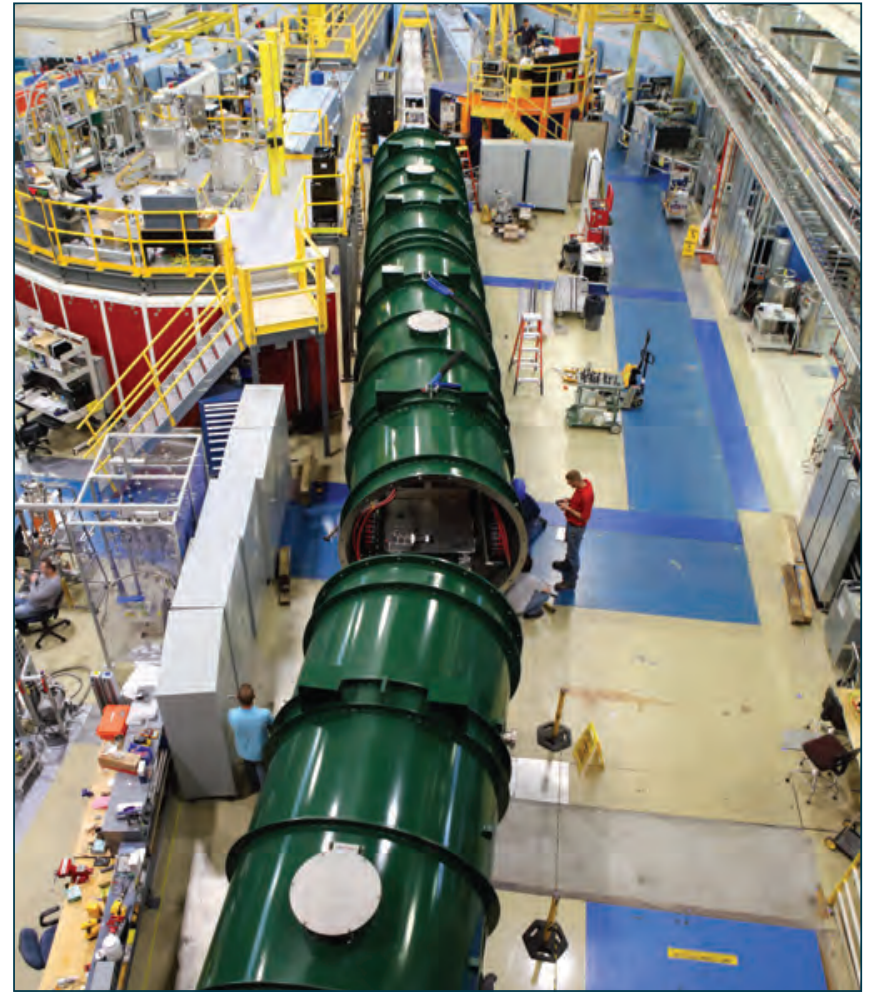

Early in 2017, the three sections of the vSANS detector vessel were moved into place, aligned, and readied for installation of the detector carriages.

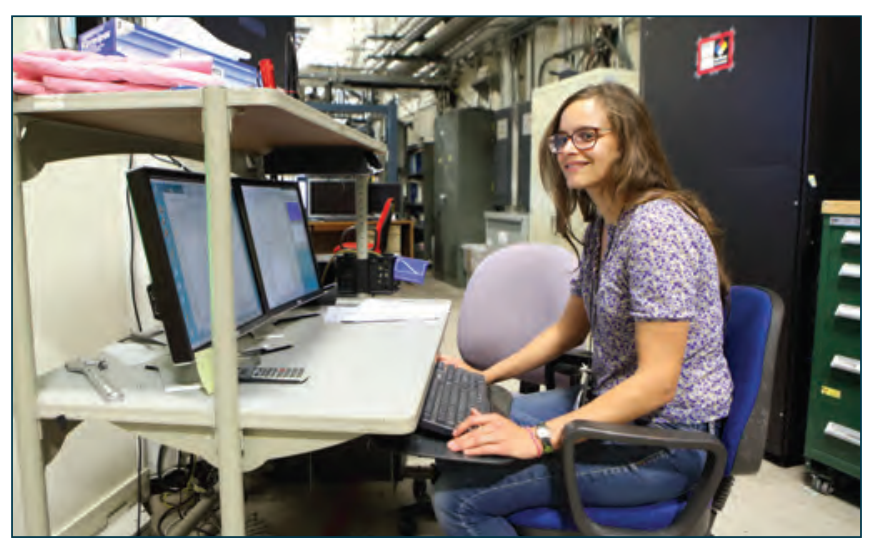

Jessica Santiago (Rice University) monitors her measurements of TiAu itinerant antiferromagnets at BT7.

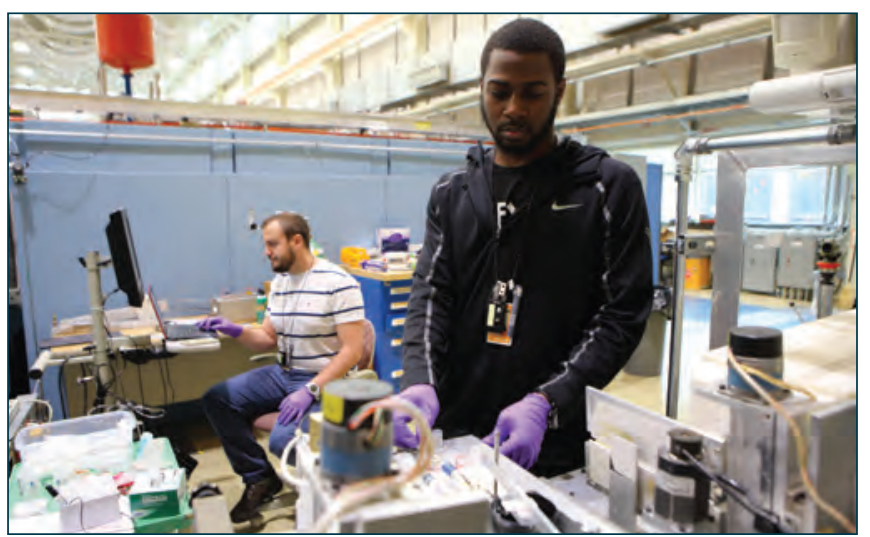

Andre Spears (front) and Joseph Dumont of Los Alamos National Laboratory mount an electrochemical cell at the NG7 reflectometer to investigate ionomers. 


\title{
Determination of functional collective motions in a protein at atomic resolution using coherent neutron scattering
}

\author{
L. Hong, ${ }^{1}$ N. Jain, ${ }^{2}$ X. L. Cheng,,$^{2,3}$ A. Bernal, ${ }^{2}$ M. Tyagi, ${ }^{4,5}$ and J. C. Smith ${ }^{2,3}$
}

$\mathrm{L}$ arge amplitude, collective atomic motions in proteins play a crucial role in many functional processes, including the entry of substrates into catalytic sites, allosteric conformational change, and enzymatic reactions. These motions have been extensively investigated using molecular dynamics (MD) simulations. However, hitherto no experimental method has been established for simultaneously determining their forms, timescales and amplitudes directly. Here, we demonstrate how dynamic coherent neutron scattering measurements collected on a perdeuterated protein can be used to derive complete description of functional collective motions. Using cytochrome P450 (CYP101) as a case study, we show that the dominant collective mode in the protein corresponds to a specific relative motion between defined segments of the protein providing substrate access to the catalytic site [1]. The experimental method developed here for the quantitative characterization of functional modes is applicable to protein systems in general.

The neutron scattering experiments were conducted on both camphor-bound CYP101 and its perdeuterated counterpart using the High Flux Backscattering Spectrometer (HFBS) and Neutron Spin Echo (NSE) spectrometers at the NCNR. We refer to these two samples as H-CYP101 and D-CYP101, respectively. The neutron signals collected from H-CYP101 are mostly incoherent signals, characterizing self-correlations in atomic motions, while the ones collected from D-CYP101 are primarily coherent signals, probing cross-correlations, i.e., inter-atomic fluctuations. A system undergoing in-phase collective motions, i.e., constituting units moving synchronously in time with similar amplitudes, will exhibit $S_{c o h}(q, \Delta E) \propto I(q) S_{\text {inc }}(q, \Delta E)$ while $S_{\text {coh }}(q, \Delta E) \propto S_{\text {inc }}(q, \Delta E)$ is found if the motion is completely random and uncorrelated $[2,3]$. Here, $I(q)$ is the overall static structure factor, and $S_{\text {coh }}(q, \Delta E)$ and $S_{\text {inc }}(q, \Delta E)$ are the coherent and incoherent dynamical structure factors collected on perdeuterated and hydrogenated protein samples, respectively. Fig. 1A presents $\gamma(q)$, the ratio between the integral of $S_{\text {coh }}(q, \Delta E)$ in the energy window 5 to $10 \mu \mathrm{eV}(\approx 100 \mathrm{ps}$ to $200 \mathrm{ps}$ ) and the corresponding integral for $S_{\text {inc }}(q, \Delta E)$ at $285 \mathrm{~K}$. As seen in Fig. 1A, the $q$ dependence of $\gamma(q)$ resembles that of $I(q)$. To verify the above result, all-atom molecular
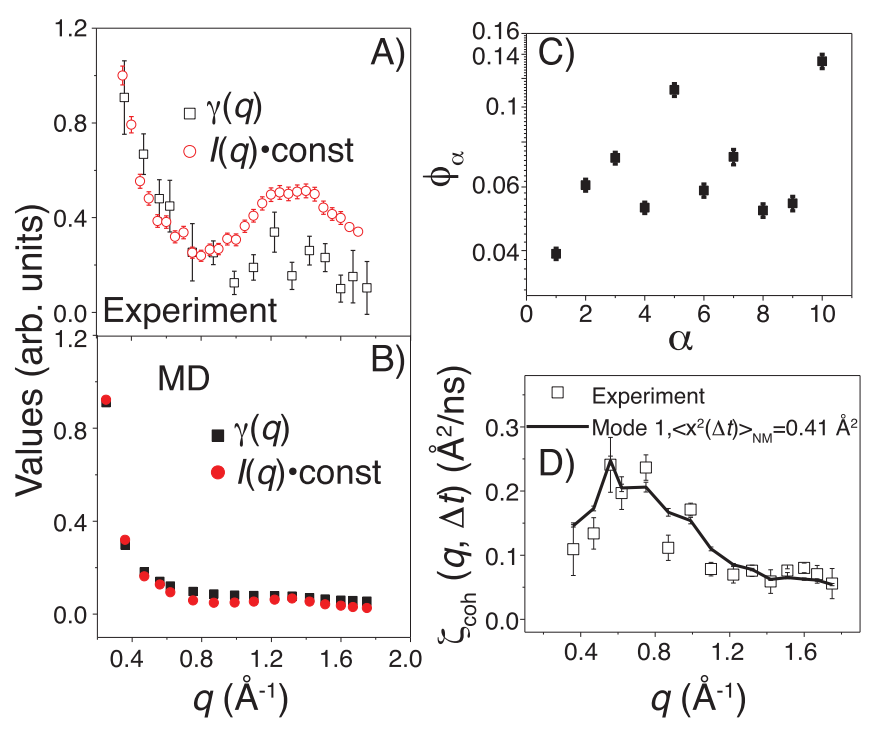

FIGURE 1: Comparison of the $q$ dependence of $\gamma(q)$ with that of $I(q)$ obtained (A) experimentally and (B) from simulation. (C) The scoring function $\phi_{\alpha}$ for different normal modes, as compared to the experimental $\zeta_{\text {coh }}(q, \Delta t)$, smaller values indicating better agreement. (D) Comparison of $\zeta_{c o h}(q, \Delta t)$ measured experimentally and that derived from the 1st Normal Mode with an amplitude of $\left\langle x^{2}\right\rangle_{\mathrm{NM}}=0.41 \AA^{2}$.

dynamics (MD) simulation was performed on CYP101 in the same conditions as the experiment, and the same analysis was performed (Fig. 1B). Again, the MD-derived $\gamma(q)$ resembles the $q$ dependence of $I(q)$. Hence, as shown by both experiment and simulation (Fig. 1), $\gamma(q)$ exhibits a $q$ dependence similar to $I(q)$ down to $0.35 \AA^{-1}(\approx 2 \mathrm{~nm})$. This demonstrates the existence of collective in-phase atomic motions across several nanometers in the protein on the time scale of $\approx 100$ ps to 200 ps.

To quantitatively characterize the spatial and temporal features of the interatomic motion in CYP101, the decay of the coherent intermediate scattering function at instrument resolution was derived as:

$$
\zeta_{c o h}(q, \Delta t)=-\frac{1 \mathrm{n} S_{c o h}(q, \Delta t)}{q^{2} \Delta t}
$$


A)

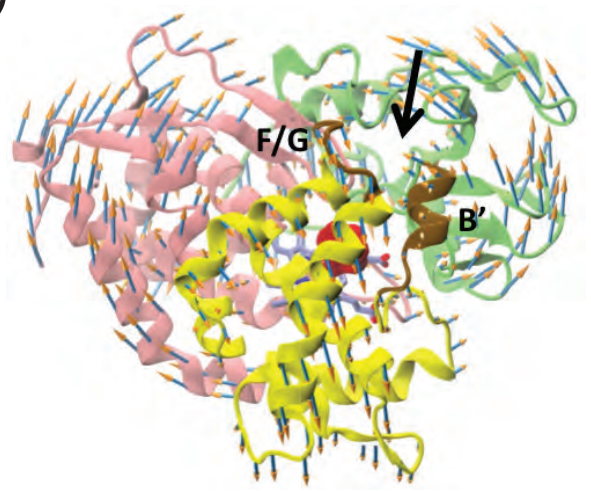

B)

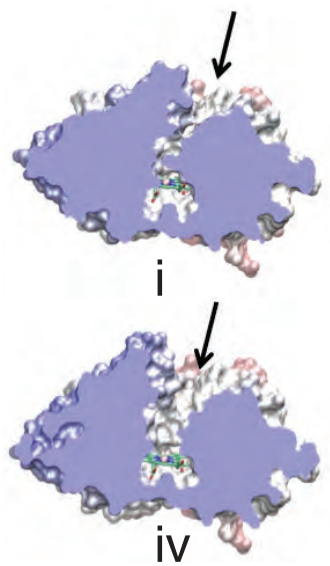

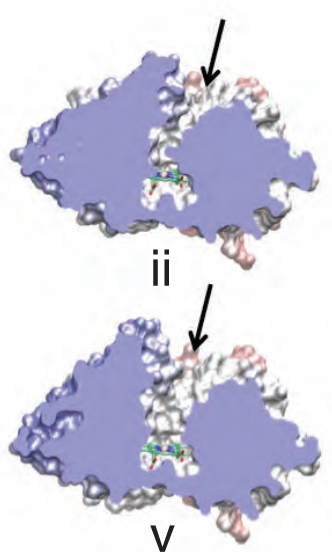

FIGURE 2: A) Three parts (marked as green, yellow, and pink) in the protein rotate against each other as for the 1st Normal Mode. The region marked red is the catalytic site. The entrance of the substrate access channel formed by the $B^{\prime}$ helices and F/G loops is marked brown. B) Cross-sectional view schematically illustrating the opening of the substrate access channel step by step via the 1st Normal Mode. The black arrow points to the entrance of the channel.

Here, $S_{\text {coh }}(q, \Delta t)$ is the intensity of the elastic peak of the coherent dynamic structure factor measured at $285 \mathrm{~K}$, where $\Delta t$ is the resolution of the instrument, $\approx 1 \mathrm{~ns}$. To determine the forms of the large-scale collective in-phase protein motions probed experimentally, we performed a normal mode analysis [4], and derived $\zeta_{c o h}(q, \Delta t)$ for each normal mode. The results (Fig. 1C) identify Mode 1 as best matching the experimental $\zeta_{\text {coh }}(q, \Delta t)$. A schematic picture of Mode 1 is presented in Fig. 2A, showing a relative rotational motion of three domains in the protein, which opens up the channel for substrates to access to the catalytic site (Fig. 2B). By fitting the experimental $\zeta_{c o h}(q, \Delta t)$ (Fig. 1D), the gate of the substrate access channel, approximated here as the distance between GLY189 in the F/G loop and ARG89 in the $B^{\prime}$ helix, is found to vary by $\approx 0.5 \AA$ on the experimental time scale of $\approx 1 \mathrm{~ns}$.

In summary, we found that collective, in-phase dynamic modes, that is, residues moving synchronously in time with similar amplitudes, span several nanometer length scales across the P450 molecule on the $\approx 100$ ps time scales. Moreover, the $q$ dependence of the elastic-neutron-scattering intensity measured on perdeuterated proteins is shown to be able to determine the most probable pathway for the collective motion in the protein molecule at atomic resolution and, furthermore, to derive the explicit amplitude of the motion on the instrumentally accessible time scale. The experimental results presented here indicate that CYP101 uses a specific collective mode in which three domains rotate against each other to create access to the catalytic site for the substrate, a mechanism crucial for the functionality of the enzyme.

\section{References}

[1] L. Hong, N. Jain, X. Cheng, A. Bernal, M. Tyagi, J. C. Smith, Science Advances 2, e1600886 (2016).

[2] J. D. Nickels, S. Perticaroli, H. O’Neill, Q. Zhang, G. Ehlers, A. P. Sokolov, Biophys. J. 105, 2182 (2013).

[3] L. Hong, N. Smolin, J. C. Smith, Phys. Rev. Lett. 112, 158102 (2014).

[4] K. Suhre, Y. H. Sanejouand, Nucleic Acids Res. 32, W610 (2004). 


\title{
Using small-angle neutron scattering to characterize the NISTmAb reference material
}

\author{
M. M. Castellanos, ${ }^{1,2}$ S. C. Howell, ${ }^{1}$ D. T. Gallagher, ${ }^{2,3}$ K. Mattison, ${ }^{4}$ S. Krueger, ${ }^{1}$ and J. E. Curtis ${ }^{1}$
}

onoclonal antibodies are the fastest growing class of therapeutic drugs used for treating challenging conditions such as cancer, immunological disorders and infectious diseases. Because of the importance of monoclonal antibodies in the pharmaceutical field, NIST recently issued the NISTmAb Reference Material, a monoclonal antibody that serves as a common framework to determine that a measurement system is working properly and assess performance of new analytical technologies. The NISTmAb has been thoroughly characterized with many conventional and novel analytical assays as described in a collection of three volumes of the book "State-of-the-Art and Emerging Technologies for Therapeutic Monoclonal Antibody Characterization" [1-3]. In our study, we use small-angle scattering to obtain information about the molecular structure of the NISTmAb in solution and characterize interactions at high protein concentrations in solution and in amorphous phases.

Small-angle neutron scattering (SANS) can provide information on the molecular structure of the NISTmAb and the spacing between proteins at different concentrations, because SANS is sensitive to the spatial relation of atoms in the sample. A scattering profile of the intensity as a function of scattering angle can be obtained for a molecule in solution. This profile can be further analyzed with theoretical and computational models to obtain information about the shape of the molecule and the distribution of molecules in concentrated systems.

In this study, experimental scattering data was used to investigate the structure of the NISTmAb in solution and the flexibility of monoclonal antibodies. Figure 1 shows a structure of the NISTmAb that is consistent with experimental data. We used computer simulations including molecular dynamics (MD) and torsion angle Monte Carlo (MC) simulations to obtain ensembles of structures that encompass the wide range of conformations that antibodies can explore. MD simulations were used to obtain a representative equilibrated structure for the $\mathrm{MC}$ simulations. From the $\mathrm{MC}$ simulations, we obtained structures that explore a wide range of configurations with radius of gyration $\left(R_{\mathrm{g}}\right)$ varying from $36 \AA$ up to $57 \AA$.

Despite the wide range of configurations, the average scattering profile of the simulated structures agrees well with the experimental data, suggesting that an ensemble of NISTmAb

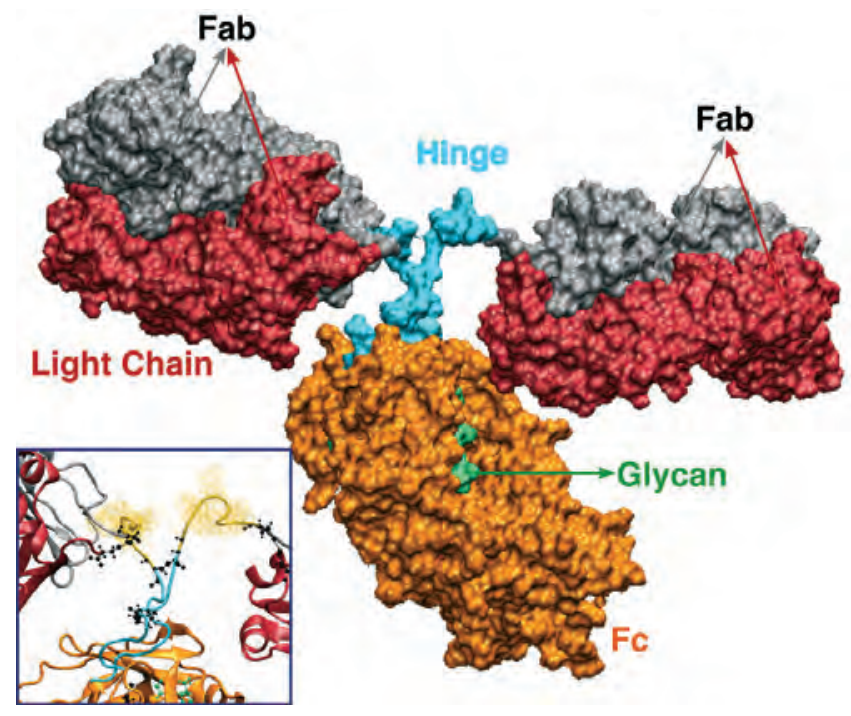

FIGURE 1: Surface representation of NISTmAb model with its corresponding regions colored as follows: Fab is represented in red (light chain) and gray (heavy chain), hinge in light blue, and Fc in orange (heavy chains) and green (glycans). In the inset, the region in yellow represent the hinge segments sampled using the MC simulations.

flexible structures is a consistent representation of the solution structure. The worst agreement with the experimental profile was observed for the most compact structure, which is about $13 \AA$ smaller than the experimental value of $49 \AA$. The agreement with experimental data for each structure is assessed using the $\chi^{2}$ parameter, which evaluates the difference in intensity between the experimental and simulated profiles while accounting for the uncertainty in the scattered intensity. The smaller the $\chi^{2}$ result the better the agreement with the experimental data.

The volumetric space occupied by atoms in ensembles of simulated structures is represented in Figure 2. In this figure, we also compare a subset of structures from MD and torsion angle $\mathrm{MC}$ simulations that have low $\chi^{2}$, high $\chi^{2}$, low $R_{\mathrm{g}}$ and high $R_{\mathrm{g}}$. These plots represent the wide range of configurations that domains of the NISTmAb can explore in solution. All the ensembles from $M C$ encompass highly flexible structures, whereas the ensembles from MD simulations mainly represent fluctuations within an initial configuration. Previous theoretical and experimental studies have suggested that bending motions

\footnotetext{
1 NIST Center for Neutron Research, National Institute of Standards and Technology, Gaithersburg, MD 20899

2 Institute for Bioscience and Biotechnology Research, Rockville, MD 20850

${ }^{3}$ Material Measurement Laboratory, National Institute of Standards and Technology, Gaithersburg, MD 20899

${ }^{4}$ Malvern Instruments, Westborough, MA 01581
} 

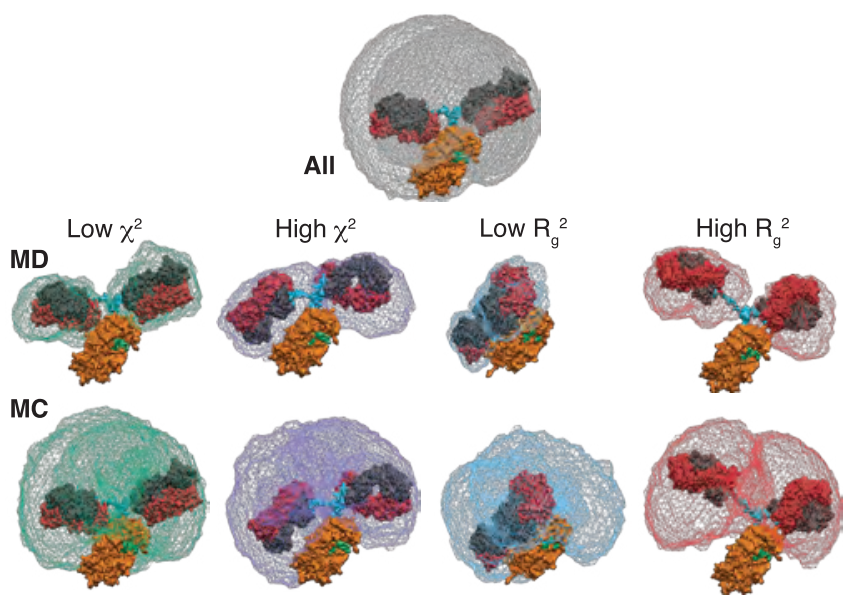

FIGURE 2: Spatial range occupied during the MC simulations (top row), the MD simulations (middle row), and the MC simulations for specific regions of interest (bottom row). In these structures, the Fc domain (bottom of structure) was used as a reference point and thus only the spatial range of the Fab domains is shown.

of domains in an antibody molecule occur in the 100 ns to $1 \mu \mathrm{s}$ time scales $[4,5]$. Thus, MD simulations alone cannot fully capture domain motions in the NISTmAb. Consequently, combining MD with MC simulations is a suitable approach to explore the known flexibility of antibody molecules.

SANS was also used to study intermolecular interactions of $\mathrm{NISTmAb}$ in solution at high concentrations with $0 \mathrm{mM}$ and $150 \mathrm{mM}(\mathrm{M}=\mathrm{mol} / \mathrm{L})$ of added $\mathrm{NaCl}$ at pD 6.4 (pH 6.0). Analysis of the SANS profiles indicate that the steric and electrostatic repulsion dictate the interactions of NISTmAb with no added $\mathrm{NaCl}$. For the samples with $150 \mathrm{mM} \mathrm{NaCl}$, although the net interaction in the system is repulsive, the electrostatic repulsion is negligible because the ions in $\mathrm{NaCl}$ screen the surface charges of the NISTmAb.

SANS is not only suitable to study liquid solutions, but it can also be used to investigate in situ the morphology in amorphous glasses (frozen samples and powders) during freezing and thawing. Figure 3 shows the SANS profiles of frozen and thawed NISTmAb samples in buffer with $0 \mathrm{mM}$ and $150 \mathrm{mM} \mathrm{NaCl}$. Because of the screening of electrostatic charges observed in the presence of ions, samples at $25^{\circ} \mathrm{C}$ with $\mathrm{NaCl}$ show higher low- $Q$ intensities compared to the profiles of samples with no $\mathrm{NaCl}$. No changes in the scattering profiles are observed when decreasing the temperature until $-10^{\circ} \mathrm{C}$. At this condition, and upon further decreasing the temperature, a protein peak is observed at $\approx 0.2 \AA$, which corresponds to a length scale of $32 \AA$. This distance corresponds to $\approx 2 / 3$ of the $R_{\mathrm{g}}$ obtained in solution, indicating that NISTmAb molecules interdigitate as a result of their flexibility and shape. Moreover, the linear upturn in the low- $Q$ region, which probes length scales larger than $200 \AA$, represents protein aggregates and ice cracks. After thawing the sample by increasing the temperature to $25^{\circ} \mathrm{C}$, the solution features of the scattering profile are fully recovered for both samples in $0 \mathrm{mM}$ and $150 \mathrm{mM} \mathrm{NaCl}$. Up to three freezing/

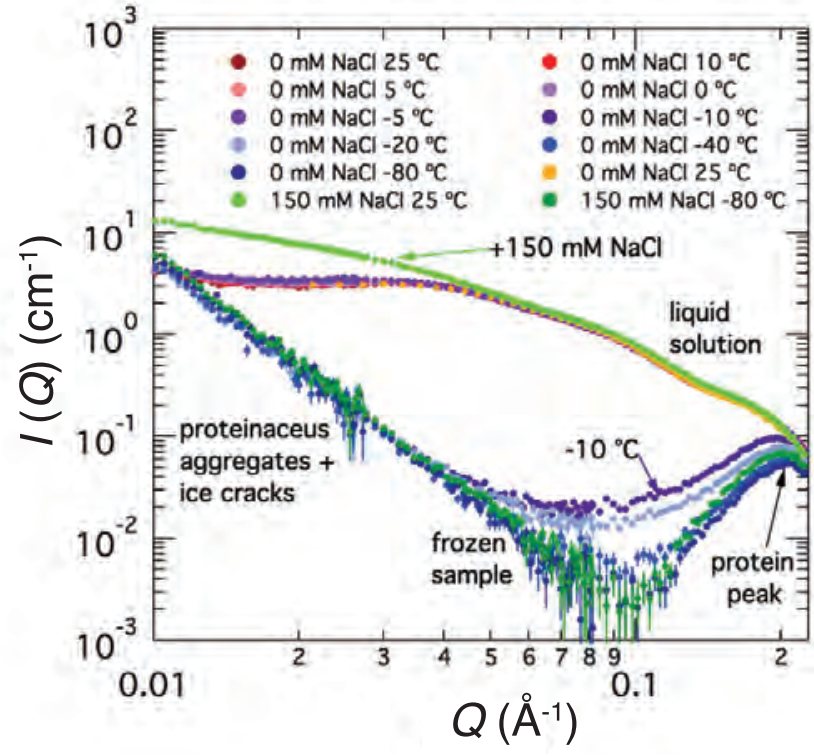

FIGURE 3: SANS profile of NISTmAb solutions during freezing and thawing cycles. Protein concentrations are $121 \mathrm{mg} / \mathrm{mL}$ and $141 \mathrm{mg} / \mathrm{mL}$ for the $0 \mathrm{mM}$ and $150 \mathrm{mM} \mathrm{NaCl}$ samples respectively.

thawing cycles were performed with no changes in the scattering profile, indicating that the overall structure of the NISTmAb is fully recovered.

In conclusion, SANS has been used to study the conformational flexibility, structure and protein-protein interactions of monoclonal antibodies in a wide range of conditions. Smallangle scattering experiments and molecular simulations provide insights on the configuration space sampled by the NISTmAb in solution. Obtaining an ensemble of representative structures that explore the flexibility of antibodies was only possible with a combined MD and MC simulation approach. Upon comparing the simulated profiles with experimental data, a unique structure that describes the experimental scattering profile was not found. On the contrary, ensembles of flexible structures provide an average scattering profile that agrees with the experimental data in solution. Moreover, SANS was suitable to assess the interactions of NISTmAb at high protein concentrations. Crowding was observed during freezing and thawing cycles of NISTmAb samples, where molecular interdigitation occurs due to the flexible structure and shape of the NISTmAb.

\section{References}

[1] J. E. Schiel, D. L. Davis, O. V. Borisov. ACS Symposium Series Vol. 1, ix-xi (2014).

[2] J. E. Schiel, D. L. Davis, O. V. Borisov. ACS Symposium Series Vol. 2, ix-xii (2015).

[3] J. E. Schiel, D. L. Davis, O. V. Borisov. ACS Symposium Series Vol. 3, ix-x (2015).

[4] J. Yguerabide, H. F. Epstein, L. Stryer. J. Mol. Biol. 51, 573 (1970).

[5] J. A. McCammon, M. Karplus. Nature 268, 765 (1977). 


\title{
From serendipity to rational design: tuning the blue trigonal bipyramidal $\mathrm{Mn}^{3+}$ chromophore to violet and purple through application of chemical pressure
}

\author{
J. Li, ${ }^{1}$ S. Lorger,${ }^{1}$ J. K. Stalick, ${ }^{2}$ A. W. Sleight, ${ }^{1}$ and M. A. Subramanian ${ }^{1}$
}

$\mathrm{T}$ he desire for stable pigments has existed for thousands of years. The recent unexpected discovery of a $\mathrm{Yln}_{1-\mathrm{x}} \mathrm{Mn}_{\mathrm{x}} \mathrm{O}_{3}$ solid solution with an intense blue color was the first example of an oxide with the blue chromophore based on a $\mathrm{Mn}^{3+}$ ion in trigonal bipyramidal (TBP) coordination [1]. These new oxides surpass the existing synthetic blue pigments in many aspects: brighter color, higher stability, better near infrared (NIR) reflectance and more environmentally benign. They are now considered as the new Blue Standard and have attracted tremendous public attention from diverse backgrounds. This discovery opened up a new field of research: rational design of novel inorganic pigments through cation substitution into the TBP sites of the host oxides. A complete $\mathrm{Y} I \mathrm{n}_{1-x} \mathrm{Mn}_{\mathrm{x}} \mathrm{O}_{3}$ solid solution could be prepared despite the size mismatch between $\mathrm{In}^{3+}$ and $\mathrm{Mn}^{3+}$ due to the similar $\mathrm{In}-\mathrm{O}$ and $\mathrm{Mn}-\mathrm{O}$ basal-plane distances in isostructural $\mathrm{YlnO}_{3}$ and $\mathrm{YMnO}_{3}$. Given the flexibility of this hexagonal crystal structure (Fig.1) we have been able to create a "rainbow" of colors through rational design as shown in Figure 2. Starting from the parent compound $\mathrm{YlnO}_{3}$, substituting a small amount of iron for indium produces intense orange colors [2], and replacing some or all indium with titanium and copper makes bright green colors [3]. By substituting some $\mathrm{In}^{3+}$ in $\mathrm{Y}(\mathrm{In}, \mathrm{Mn}) \mathrm{O}_{3}$ with equal amounts of $\mathrm{Ti}$ and $\mathrm{Zn}$, we reported recently [4] a range of violet to purple colors (Figs.1-2).
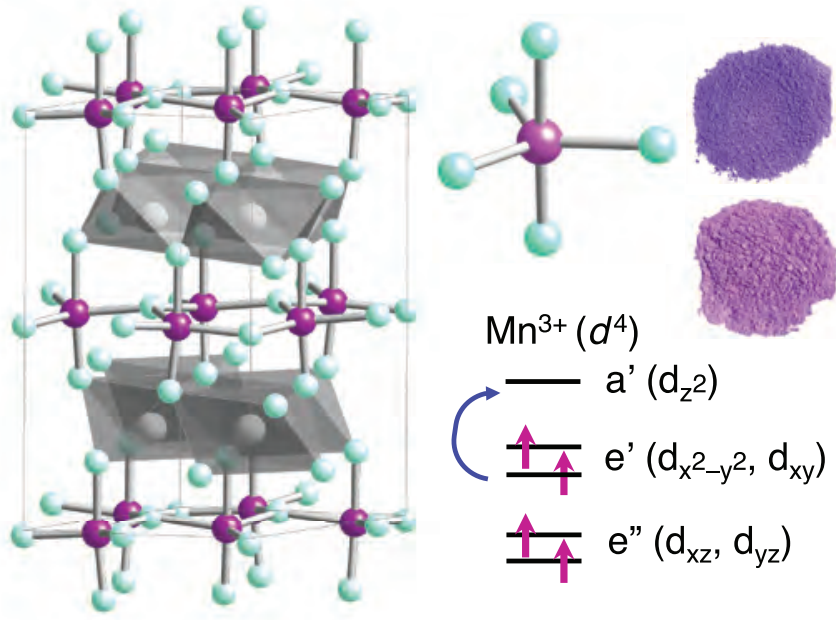

FIGURE 1: Hexagonal structure of $\mathrm{YMO}_{3}(\mathrm{M}=\mathrm{In} / \mathrm{Mn} / \mathrm{Ti} / \mathrm{Zn} / \mathrm{Al}$, purple; 0 , cyan; $\mathrm{Y}$, gray). Schematic energy levels for the spin-up $\mathrm{Mn}^{3+} 3 \mathrm{~d}$ orbitals in trigonal bipyramidal coordination are shown. The general formula of the selected violet and purple compositions (top right) is $\mathrm{YIn}_{1-\mathrm{x}-2 \mathrm{y}-\mathrm{z}} \mathrm{Mn}_{\mathrm{x}} \mathrm{Ti}_{\mathrm{y}} \mathrm{Zn}_{\mathrm{y}} \mathrm{Al}_{\mathrm{z}} \mathrm{O}_{3}$.

Historically, violet and purple colors have long been associated with royalty, aristocracy, piety, and faith. Although all suffer from environmental and/or durability issues, traditional violet and purple pigments such as Chinese Han purple $\left(\mathrm{BaCuSi}_{2} \mathrm{O}_{6}\right)$, ultramarine violet $\left(\mathrm{Na}_{6-10} \mathrm{Al}_{6} \mathrm{Si}_{6} \mathrm{O}_{24} \mathrm{~S}_{2-4}\right)$, manganese violet $\left(\mathrm{NH}_{4} \mathrm{MnP}_{2} \mathrm{O}_{7}\right)$, and cobalt violet $\left(\mathrm{CO}_{3}\left(\mathrm{PO}_{4}\right)_{2}\right)$ are still commonly used inorganic pigments.

In the current study [4], polycrystalline pigment samples were prepared by conventional solid-state reactions at $1300^{\circ} \mathrm{C}$. Starting from the composition $\mathrm{YIn}_{1-x} \mathrm{Mn}_{\mathrm{x}} \mathrm{O}_{3}$ with low $\mathrm{Mn}$ content $(x \leq 0.2)$, the intense blue color was tuned to various shades of violet and purple colors by substituting different amounts of $\mathrm{Ti} / \mathrm{Zn} / \mathrm{Al}$ for In. The solid solutions of $\mathrm{YIn}_{1-\mathrm{x}-2 \mathrm{z}-\mathrm{Z}} \mathrm{Mn}_{\mathrm{x}} \mathrm{Ti}_{\mathrm{y}} \mathrm{Zn}_{\mathrm{y}} \mathrm{Al}_{\mathrm{z}} \mathrm{O}_{3}$ $(x=0.005 \sim 0.2, y=0.1 \sim 0.4, z=0 \sim 0.1)$ were synthesized phase pure up to $y=0.3$ and $z=0.1$. XRD study shows cell edges $a, c$, cla ratio, and cell volume all decrease with increasing substitution due to the smaller ionic radii of trivalent $\mathrm{Mn} / \mathrm{Ti} / \mathrm{Zn} / \mathrm{Al}$ compared with that of In, which confirms the formation of the solid solution.

The blue color of $\mathrm{YIn}_{1-\mathrm{x}} \mathrm{Mn}_{\mathrm{x}} \mathrm{O}_{3}$ is presumably associated with the crystal field splitting of TBP coordination (Fig.1) and the short apical $\mathrm{Mn}-\mathrm{O}$ bonds [1]. To understand the color alteration or origin of the violet and purple colors, it is important to determine precisely the atomic positions of $\mathrm{Mn}$ and oxygen using neutron diffraction. Powder diffraction data were collected on the NIST high-resolution diffractometer BT-1, and the structure refinements were based on the acentric space group $P \sigma_{3} \mathrm{~cm}$.

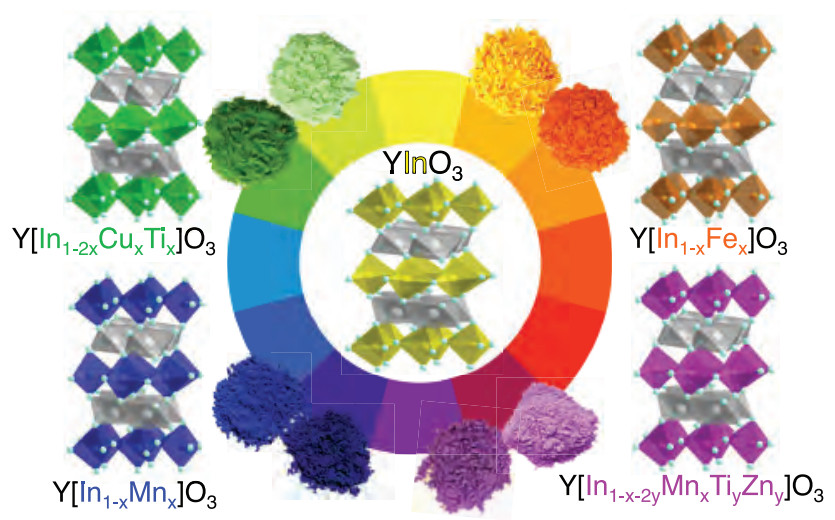

FIGURE 2: A range of colors are created through TBP site substitutions in hexagonal $\mathrm{YInO}_{3}$. 


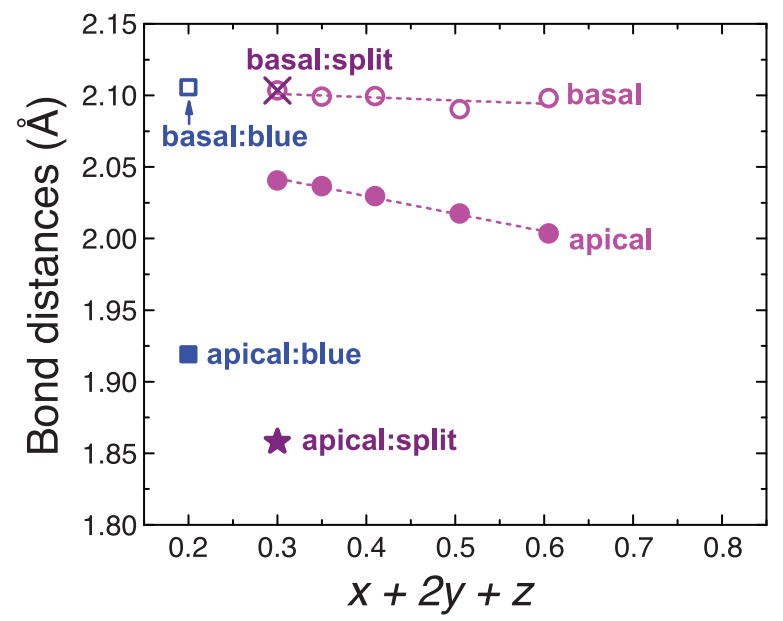

FIGURE 3: Average bond distances of purple $\mathrm{Yln}_{1-\mathrm{x}-2 \mathrm{y}-\mathrm{z}} \mathrm{Mn}_{\mathrm{x}} \mathrm{TiyZ} \mathrm{n}_{\mathrm{y}} \mathrm{Al}_{z} \mathrm{O}_{3}$ and blue $Y \mathrm{In}_{0.8} \mathrm{Mn}_{0.2} \mathrm{O}_{3}$ samples. Open and filled circles are distances refined for purple samples without local relaxation. The cross sign and star (purple), and the open and filled squares (blue), are distances refined with split sites for In and $\mathrm{Mn}$ and two apical oxygens. The value of $(x+2 y+z)$ is the total TBP site substitution for In.

We found that for a simple system like blue $\mathrm{Yln}_{0.8} \mathrm{Mn}_{0.2} \mathrm{O}_{3}$, we could refine In and $\mathrm{Mn}$ positions separately and refine the separate positions for the apical $\mathrm{O}$ atoms associate with $\mathrm{In}$ and $\mathrm{Mn}$ [5]. Such an approach becomes much more challenging for the purple $\mathrm{Y}(\mathrm{In}, \mathrm{Mn}, \mathrm{Ti}, \mathrm{Zn}, \mathrm{Al}) \mathrm{O}_{3}$ compositions with relatively low Mn content. A split model was, however, successful for $Y \mathrm{In}_{0.7} \mathrm{Mn}_{0.1} \mathrm{Ti}_{0.1} \mathrm{Zn}_{0.1} \mathrm{O}_{3}$. This was accomplished by refining the positions of $\mathrm{Mn}$ and its apical oxygens separately, leaving $\mathrm{In}$, Ti, $\mathrm{Zn}$, and $\mathrm{Al}$ at one site with occupancies fixed according to the nominal composition. Such a refinement is facilitated by the negative neutron scattering length of $\mathrm{Mn}$. Figure 3 displays a clear contraction of the apical bond distances with increasing substitution and a minor change in the basal plane distances.

Without allowing local relaxation for $\mathrm{Mn}$, the average apical Mn-O distance is refined to be $2.04 \AA$, but it becomes much shorter (1.86 $\AA$ ) when refined with the split model. The same distance is $1.92 \AA$ for blue $\mathrm{YIn}_{0.8} \mathrm{Mn}_{0.2} \mathrm{O}_{3}$ refined with local relaxation for $\mathrm{Mn}$, indicating a decreasing apical $\mathrm{Mn}-\mathrm{O}$ bond distance going from blue to purple. The actual difference between apical $\mathrm{Mn}-\mathrm{O}$ distances of blue and purple samples might be even bigger if local relaxation could be applied for all the TBP cations. The decrease in apical $\mathrm{Mn}-\mathrm{O}$ bond distance is presumably responsible for the color shift from blue to purple considering that the energy of the $d_{x^{2}}$ state relative to the valence band maximum is determined by the $\mathrm{Mn}-\mathrm{O}$ apical bond length (Fig.1). Since the apical distances vary much more than the basal plane distances do, it is primarily the apical $\mathrm{Mn}-\mathrm{O}$ distances that correlate with the position of the allowed $d-d$ transition.

Diffuse reflectance spectra (Fig. 4) of $\mathrm{Y}_{1-x-2 y} \mathrm{Mn}_{\mathrm{x}} \mathrm{Ti}, \mathrm{Zn} \mathrm{n}_{\mathrm{y}} \mathrm{O}_{3}$ show that with increasing concentration of $\mathrm{Ti} / \mathrm{Zn}$, the lower-energy absorption peak is blue-shifted by $0.1 \mathrm{eV}$ to $0.4 \mathrm{eV}$ and the onsets of the higher-energy absorption peak is red-shifted by $0.1 \mathrm{eV}$ to $0.4 \mathrm{eV}$ with respect to the blue. We assign the lower energy absorption to the allowed $d$-d transition $\left(e^{\prime}\left(d_{x^{2}-y^{2}}, d_{x y}\right)\right.$ to $\left.a^{\prime}\left(d_{z^{2}}\right)\right)$ as shown in Figure 1 and the absorption in the near-UV to a

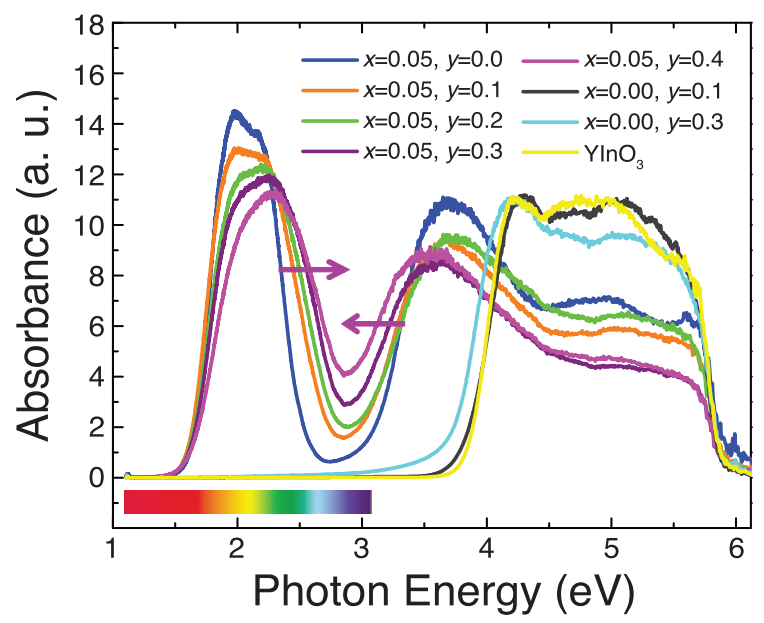

FIGURE 4: Diffuse reflectance spectra for selected purple samples $\mathrm{Yln}_{1-\mathrm{x}-2 \mathrm{y}} \mathrm{Mn}_{\mathrm{x}} \mathrm{Ti}_{\mathrm{y}} Z \mathrm{Zn}_{\mathrm{y}} \mathrm{O}_{3}(x=0.05, y=0.1 \sim 0.4)$. Blue $\mathrm{YIn}_{0.95} \mathrm{Mn}_{0.05} \mathrm{O}_{3}, \mathrm{YlnO}_{3}$ and compositions without Mn chromophore $(x=0.00, y=0.1$ and 0.3$)$ are included for comparison.

charge-transfer transition from $\mathrm{O}$ to $\mathrm{Mn}$. These peaks also broaden because of the disorder around $\mathrm{Mn}$ with respect to $\mathrm{In}$, Ti and $\mathrm{Zn}$ near neighbors. The color is defined by the minimum absorption, and that shifts to higher energy with increasing $y$. In comparison to blue $\mathrm{YIn}_{0.95} \mathrm{Mn}_{0.05} \mathrm{O}_{3}$ the shorter $\mathrm{Mn}-\mathrm{O}$ distances impact both the $d-d$ transition and the charge transfer peaks.

In addition to color, other desired properties of pigments are the durability and the ability to reflect heat. Treating our pigments under severe acid and thermal conditions resulted in negligible weight and color changes. NIR measurements for all our samples show unusually high reflectance in the range of $700 \mathrm{~nm}$ to $2500 \mathrm{~nm}$ comparable to $\mathrm{TiO}_{2}$.

In summary, we demonstrate with the $\mathrm{YlnO}_{3}-\mathrm{YMnO}_{3}$ system that colors can be designed by manipulating the crystal structure, in this case the local environment of the $\mathrm{Mn}^{3+}$ chromophore in trigonal bipyramidal coordination. Our novel, nontoxic violet/ purple pigments outperform the existing synthetic pigments in durability, thermal stability, NIR reflectance, and hence energysaving properties. We expect that our results may lead to routes for the development of inexpensive, earth-abundant based, environmentally benign, and highly stable inorganic pigments.

\section{References}

[1] A. E. Smith, H. Mizoguchi, K. Delaney, N. A. Spaldin, A. W. Sleight, M. A. Subramanian, J. Am. Chem. Soc. 131, 17084 (2009).

[2] P. Jiang, J. Li, A. W. Sleight, M. A. Subramanian, Inorg. Chem. 50, 5858 (2011).

[3] A. E Smith, A. W. Sleight, M. A. Subramanian, Mater. Res. Bull. 46, 1 (2011).

[4] J. Li, S. Lorger, J. K. Stalick, A. W. Sleight, M. A. Subramanian, Inorg. Chem. 55, 9798 (2016).

[5] J. Li, A. W. Sleight, M. A. Subramanian, Chem. Mater. 28, 6050 (2016). 


\section{Capture and release of chlorine and bromine gas with a hybrid sponge}

Y. Tulchinsky, ${ }^{1}$ C. H. Hendon, ${ }^{1}$ K. A. Lomachenko, ${ }^{2,3}$ E. Borfecchia, ${ }^{4}$ B. C. Melot, ${ }^{5}$ M. R. Hudson, ${ }^{6}$ J. D. Tarver, ${ }^{6,7}$ M. D. Korzyński, ${ }^{1}$ A. W. Stubbs, ${ }^{1}$ J. J. Kagan, ${ }^{8}$ C. Lamberti, ${ }^{3,4}$ C. M. Brown, ${ }^{6}$ and M. Dincă ${ }^{1}$

I treme toxicity, corrosiveness, and volatility pose serious challenges for the safe storage and transportation of elemental chlorine and bromine, $\mathrm{Cl}_{2}$ and $\mathrm{Br}_{2}$, respectively. Yet, these halogens play critical roles in the chemical industry as water sanitizers, flame retardants, and key functional groups in pharmaceuticals. Metal organic frameworks (MOFs), with their demonstrated utility in storing both inert and reactive gases, seem like logical choices to address the storage challenge. However, despite thousands of reports of gas sorption and storage in MOFs, many including oxidizing or corrosive gases such as $\mathrm{O}_{2}, \mathrm{NO}, \mathrm{SO}_{2}$, and $\mathrm{NH}_{3}$, reversible storage of $\mathrm{Br}_{2}$ or $\mathrm{Cl}_{2}$ remains evasive. The lack of reports on reversible storage of the gaseous halogens (i.e., excluding $\mathrm{I}_{2}$ ) in MOFs is staggering given the interest in the field, but highlights the difficulty in isolating materials that can reversibly bind halogens and release them over at least several cycles.

One approach to capturing elemental species is to harness redox reactions between the absorbate and MOF. Here we report such a mechanism [1], where elemental $\mathrm{Br}_{2}$ and $\mathrm{Cl}_{2}$ reversibly oxidize $\mathrm{Co}$ (II) centers in $\mathrm{CO}_{2} \mathrm{Cl}_{2} \mathrm{BTDD}$ (BTDD = bis $(1 \mathrm{H}-1,2,3$ triazolo[4,5-b],[4,5-i])dibenzo[1,4]dioxin), a permanently porous MOF, to form terminal Co(III)-halides, $\mathrm{CO}_{2} \mathrm{Cl}_{2} \mathrm{X}_{2}$ BTDD $(\mathrm{X}=\mathrm{Cl}, \mathrm{Br}$ ), as evidenced crystallographically. We complete the release of the elemental halogen through thermal back-reduction to Co(II) and concomitant liberation of $X_{2}$. These results represent the first example of reversible halogen uptake and release with a MOF and herald applications for halogen capture in the context of toxic gas removal, and storage in the context of safe handling of halogens.

The olive-green colored solid, $\mathrm{CO}_{2} \mathrm{Cl}_{2} \mathrm{BTDD}$ is highly crystalline [2], however the crystallites were too small to be characterized by single crystal X-ray diffraction. Structural analysis using the Rietveld method of neutron powder diffraction (NPD) data obtained from the activated bare sample at $10 \mathrm{~K}$ revealed a three-dimensional structure exhibiting one-dimensional channels arranged in a honeycomb fashion (Figure 1) with pores that are solvent free. The inorganic regions consist of infinite $-(\mathrm{Co}-\mathrm{Cl})_{n}-$ chains interconnected by the bis-triazolate linkers. Unlike the analogous solvated Mn-containing structure where there is a disorder of the ligands about a mirror-plane symmetry element in the $R-3 m$ space

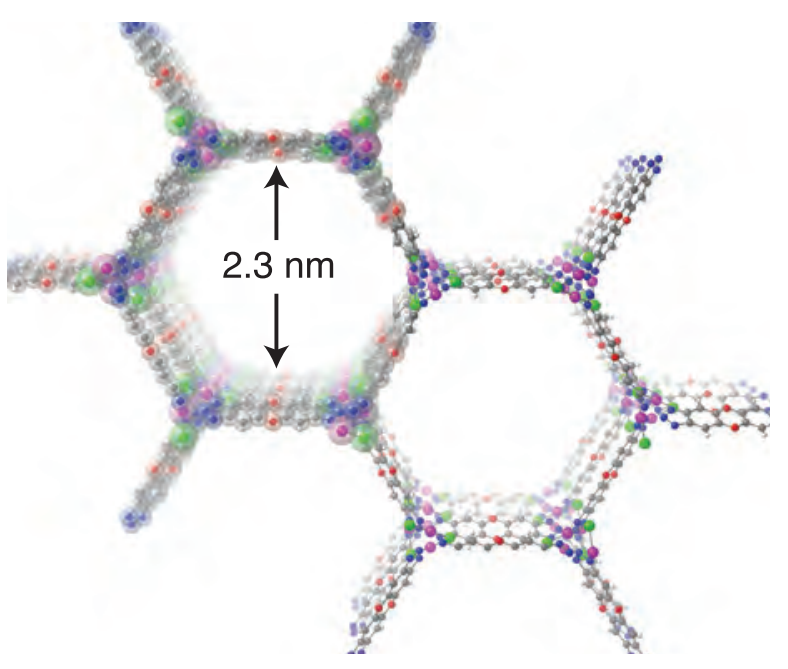

FIGURE 1: A portion of the structure of the parent $\mathrm{Co}$ (II) MOF $\mathrm{CO}_{2} \mathrm{Cl}_{2} \mathrm{BTDD}$ projected along the $c$-axis. Unsaturated cobalt ions are exposed to the pores down the 1-dimensional chain axis. Gray, red, blue, green and pink represent carbon, oxygen, nitrogen, chlorine, and cobalt, respectively.

group [2], it was determined that the optimal space group is $R-3$ with fully occupied ligand atoms at single symmetric positions.

Reaction of the bare material with gaseous $\mathrm{Cl}_{2}$ resulted in rapid oxidation and quantitative formation of dark brown microcrystals of $\mathrm{CO}_{2} \mathrm{Cl}_{4} \mathrm{BTDD}$ and crystallinity was maintained. Elemental analysis suggested stoichiometric uptake of halogen, and X-ray absorption near-edge spectra (XANES) at the Co K-edge revealed that the absorption edge of is shifted by approximately $2 \mathrm{eV}$ to higher energy relative to the parent, indicating oxidation of the $\mathrm{Co}(\mathrm{II})$ centers. Elemental $\mathrm{Br}_{2}$ is equally effective in oxidizing the MOF. Prolonged exposure to $\mathrm{Br}_{2}$ vapors results in quantitative conversion to $\mathrm{CO}_{2} \mathrm{Cl}_{2} \mathrm{Br}_{2} \mathrm{BTDD}$ as confirmed by micro-elemental analysis and inductively-coupled plasma mass spectrometry. NPD analysis of $\mathrm{Cl}_{2}$-reacted MOF revealed decreases of $0.13 \AA$ and $0.14 \AA$ in the average $\mathrm{Co}-\mathrm{N}$ and $\mathrm{Co}-\mathrm{Cl}$ bond lengths, respectively, relative to the parent, as would be expected upon oxidation of $\mathrm{Co}(\mathrm{II})$ sites to $\mathrm{Co}(\mathrm{III})$. Similarly, the MOF-Br has shorter average Co-N and Co-Cl bonds relative to the parent: $1.92 \AA$ vs. $2.12 \AA$

\footnotetext{
${ }^{1}$ Massachusetts Institute of Technology, Cambridge, MA 02139

2 European Synchrotron Radiation Facility, 71 Avenue des Martyrs, CS 40220, 38043 Grenoble Cedex 9, France

${ }^{3}$ Southern Federal University, Zorge Street 5, 344090 Rostov-on-Don, Russia

${ }^{4}$ University of Turin, Via Quarello 15, I-10135 Torino, Italy

5 University of Southern California, Los Angeles, CA 90089

${ }^{6}$ NIST Center for Neutron Research, National Institute of Standards and Technology, Gaithersburg, MD 20899

${ }^{7}$ National Renewable Energy Laboratory, Golden, C0 80401

${ }^{8}$ Weizmann Institute of Science, 234 Herzl St., Rehovot, Israel
} 


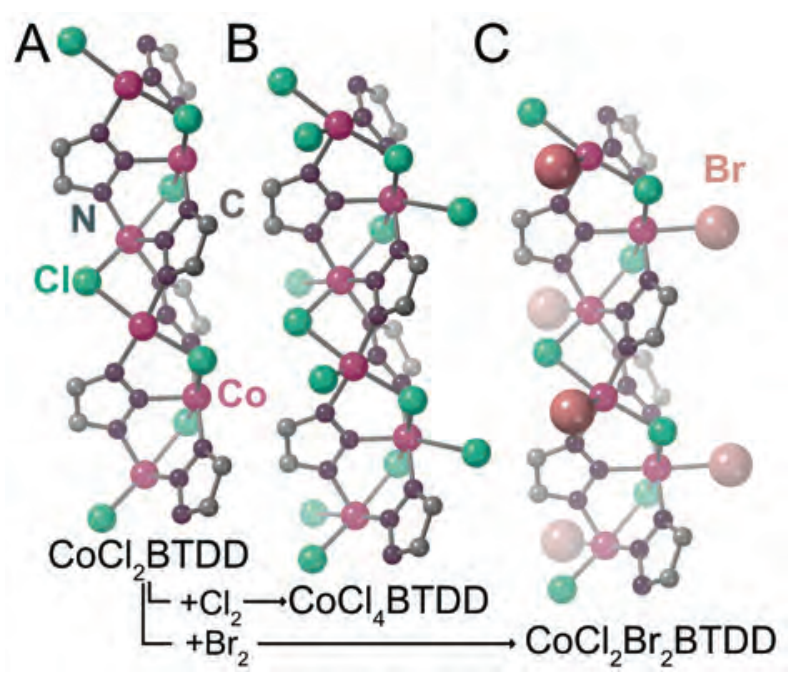

FIGURE 2: A), B), C) Partial structures along the chain axis detailing the local coordination environments of the $\mathrm{Co}$ centers in bare, $\mathrm{Cl}$ and $\mathrm{Br}$ structures, respectively, as determined by neutron powder diffraction.

and $2.34 \AA$ vs. $2.40 \AA$, respectively (Figure 2). Fourier difference mapping confirmed that the void spaces in the pores of both materials were indeed evacuated and free of excess scattering density and that only scattering for the framework skeleton and the terminal Co(III)-bound halide atoms were present.

Quantitative oxidation of the MOF was confirmed by allowing free occupancy of the terminal bromide site in the Rietveld refinement process, which gave best fits when the occupancy was $100 \%$. To further attempt to account for any possibility in mixing of $\mathrm{Cl}$ at the $\mathrm{Br}$ sites, and vice-versa, refinements were performed with a mixed $\mathrm{Cl} / \mathrm{Br}$ occupation on each individual site and simultaneously at both sites. All refinements resulted in significantly worse refinements and goodness-of-fit parameters which, importantly, revealed no structural disorder between the terminal bromides and the bridging chlorides in the oxidized material.

Thermogravimetric analysis (TGA) of chloride and bromide oxidized MOFs under a stream of He revealed surprisingly sharp weight loss steps at approximately $275^{\circ} \mathrm{C}$ and $195^{\circ} \mathrm{C}$, respectively, and no subsequent weight losses up to approximately $450^{\circ} \mathrm{C}$ (Figure 3). The parent compound itself did not exhibit any weight loss up to $400{ }^{\circ} \mathrm{C}$. This data suggested that the $\mathrm{Cl}_{2}$ and $\mathrm{Br}_{2}$ exposed MOFs may eliminate elemental halogens thermally. Coupling the thermogravimetric analyzer with an in-line mass spectrometer confirmed this hypothesis and we note that thermal elimination of $\mathrm{X}_{2}$ occurs without notable losses in crystallinity or porosity.

The weight losses observed under the milder TGA conditions, 9.5 mass \% and 21 mass \% for MOF-Cl and MOF-Br, respectively, correspond to elimination of only $70 \%$ to $80 \%$ of the theoretical halogen content. Thermal treatment of bulk samples of $\mathrm{MOF}-\mathrm{Cl}$ and $\mathrm{MOF}-\mathrm{Br}$ under vacuum gave products with elemental formulas of $\mathrm{CO}_{2} \mathrm{Cl}_{2.4} \mathrm{BTDD}$ and $\mathrm{CO}_{2} \mathrm{Cl}_{2} \mathrm{Br}_{0.34} \mathrm{BTDD}$, respectively, which also indicate loss of only approximately $80 \%$ of the releasable halogen content. lodometric titration of $\mathrm{Br}_{2}$ trapped from thermal treatment of MOF-Br confirmed that the isolated yield of $\mathrm{Br}_{2}$ under these conditions is close to $80 \%$. Importantly, the parent MOF can store

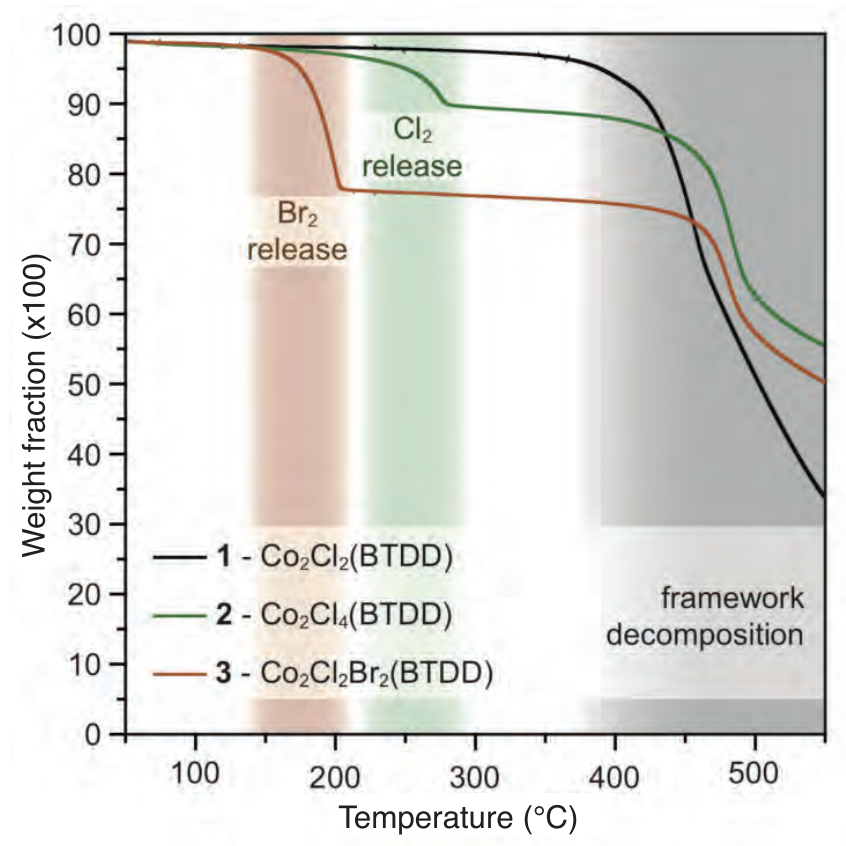

FIGURE 3: Thermogravimetric analyses. The TGA plot of the parent MOF (1: black line) shows that framework decomposition occurs at ca. $400^{\circ} \mathrm{C}$. Abrupt weight losses corresponding to halogen liberation from MOF-Cl (2-green line) and MOF- $\mathrm{Br}$ (3-brown line) are emphasized in the green and brown regions, respectively.

and release halogens more than once: three consecutive cycles of exposing $\mathrm{CO}_{2} \mathrm{Cl}_{2} \mathrm{BTDD}$ to $\mathrm{Br}_{2}$ vapors and thermal $\mathrm{Br}_{2}$ release gave a reproducible recovery of $75 \%$ to $80 \%$ of the theoretical $\mathrm{Br}_{2}$ yield with retention of structural integrity, crystallinity, and only minimal deterioration of porosity, as confirmed by SEM, PXRD analysis, and $\mathrm{N}_{2}$ adsorption measurements after each cycle, respectively. Altogether, these data suggest that a recovery yield of $80 \%$ is what should be expected in practice when using $\mathrm{CO}_{2} \mathrm{Cl}_{2} \mathrm{BTDD}$ for the reversible storage of halogens for the first cycle, with the yield becoming essentially quantitative on subsequent cycles.

In summary, uptake of elemental halogens, $\mathrm{Cl}_{2}$ and $\mathrm{Br}_{2}$, by a robust Co(II) azolate MOF occurs without loss of crystallinity or porosity. Subsequent thermal treatment of the oxidized materials releases the elemental halogens, closing a cycle that amounts to reversible capture and release of these highly corrosive gases. This cycling behavior is unprecedented in MOFs, which are typically decomposed by or react irreversibly with the lighter halogens. The unusual reactivity here is enabled by the quantitative formation of the oxidized $\mathrm{Co}(\mathrm{III})$ species, also unique for MOFs, and the facile cleavage of $\mathrm{Co}(\mathrm{III})-\mathrm{X}$ bonds to reform $\mathrm{X}_{2}$. These results provide a blueprint for the design of other porous materials geared towards the capture and storage of noxious, corrosive gases through reversible chemisorptive mechanisms.

\section{References}

[1] Y. Tulchinsky, C. H. Hendon, K. A. Lomachenko, E. Borfecchia, B. C. Melot, M. R. Hudson, J. D. Tarver, M. D. Korzyński, A. W. Stubbs, J. J. Kagan, C. Lamberti, C. M. Brown, M. Dincă, J. Am. Chem. Soc. 139, 5992 (2017).

[2] A. J. Rieth, Y. Tulchinsky, M. Dincă, J. Am. Chem. Soc. 138, 9401 (2016). 


\section{W. S. Tang, ${ }^{1,2}$ K. Yoshida, ${ }^{3}$ A. V. Soloninin, ${ }^{4}$ R. V. Skoryunov, ${ }^{4}$ O. A. Babanova, ${ }^{4}$ A. V. Skripov, ${ }^{4}$ M. Dimitrievska, ${ }^{1,5}$} V. Stavila, ${ }^{6}$ S. Orimo, ${ }^{3}$ and T. J. Udovic ${ }^{1}$

$\overline{7}$ he discovery of solid-state electrolytes with sufficiently high superionic conductivities to replace the flammable organic liquid electrolytes currently used in rechargeable batteries would substantially decrease the fire danger. We recently identified solid sodium and lithium salts of the singly charged, cage-like, bicapped-square-antiprismatic $\mathrm{CB}_{9} \mathrm{H}_{10}$ - and icosahedral $\mathrm{CB}_{11} \mathrm{H}_{12}$ - anions as having conductivities rivalling or surpassing those of commercial liquid electrolytes. However, this favorable conductivity only occurs above their inherent order-disorder phase transitions at above-ambient temperatures [1-3]. We have now been able to extend their favorable superionic conducting properties down to subambient temperatures simply by forming disordered solid-solution salt mixtures of these $\mathrm{CB}_{9} \mathrm{H}_{10}{ }^{-}$and $\mathrm{CB}_{11} \mathrm{H}_{12}$ - anions. The combination of these geometrically distinct anions appears to inhibit the formation of an ordered structure, instead leading to a disordered structure that is favorable to superionic conductivity.

$\mathrm{M}_{2}\left(\mathrm{CB}_{9} \mathrm{H}_{10}\right)\left(\mathrm{CB}_{11} \mathrm{H}_{12}\right)(\mathrm{M}=\mathrm{Li}$ or $\mathrm{Na}$ ) anhydrous compound mixtures were formed by drying aqueous solutions with equimolar amounts of $\mathrm{MCB}_{9} \mathrm{H}_{10}$ and $\mathrm{MCB}_{11} \mathrm{H}_{12}$. X-ray-diffraction (XRD) results for $\mathrm{Li}_{2}\left(\mathrm{CB}_{9} \mathrm{H}_{10}\right)\left(\mathrm{CB}_{11} \mathrm{H}_{12}\right)$ confirmed the formation of two equally prevalent disordered phases, one hexagonal (see Fig. 1),

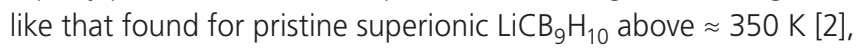
and another fCC, like that found for pristine superionic $\mathrm{LiCB}_{11} \mathrm{H}_{12}$ above $\approx 395 \mathrm{~K}$ [1]. The different phases are likely due to incomplete homogenization yielding two mixture fractions, one that is slightly $\mathrm{CB}_{9} \mathrm{H}_{10}$ - rich (hexagonal) and one that is slightly $\mathrm{CB}_{11} \mathrm{H}_{12}{ }^{-}$rich ( $\left.\mathrm{fCC}\right)$. XRD results for $\mathrm{Na}_{2}\left(\mathrm{CB}_{9} \mathrm{H}_{10}\right)\left(\mathrm{CB}_{11} \mathrm{H}_{12}\right)$ also confirmed the formation of a predominant disordered hexagonal phase like that found for pristine superionic $\mathrm{NaCB}_{9} \mathrm{H}_{10}$ above $\approx 310 \mathrm{~K}$ [2]. Differential scanning calorimetry measurements for both mixtures cycled between $200 \mathrm{~K}$ and $473 \mathrm{~K}$ displayed no obvious endothermic or exothermic phase transitions, indicating that the disordered solid-solution phases are stable at least within this temperature range.

Quasielastic neutron scattering measurements were critical for establishing the temperature dependences of the anion orientational mobilities associated with the solid-solutions $\mathrm{M}_{2}\left(\mathrm{CB}_{9} \mathrm{H}_{10}\right)\left(\mathrm{CB}_{11} \mathrm{H}_{12}\right)$ compared to those for the single-anion

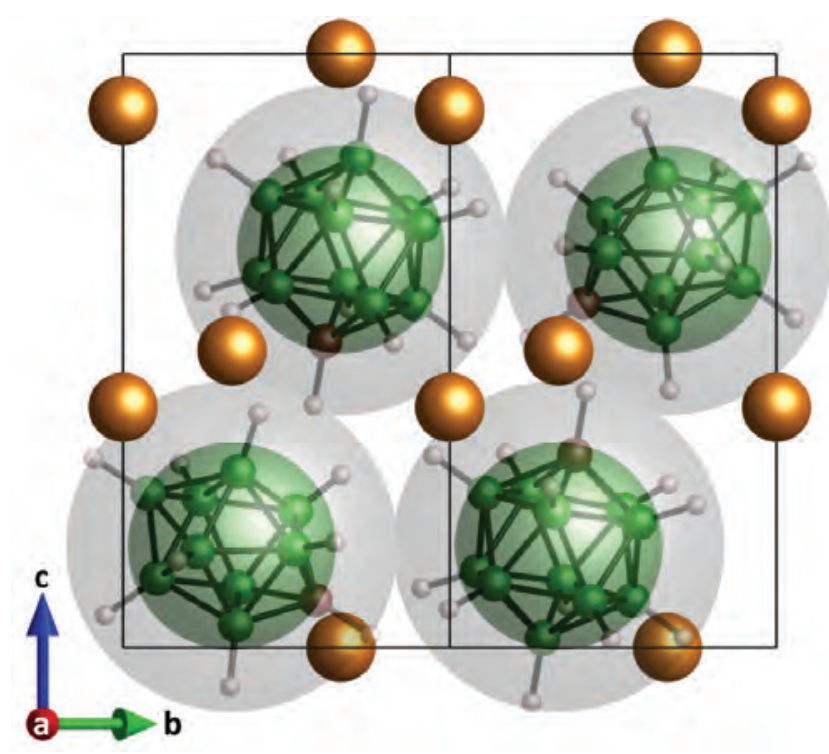

FIGURE 1: The disordered hexagonal $\mathrm{M}_{2}\left(\mathrm{CB}_{9} \mathrm{H}_{10}\right)\left(\mathrm{CB}_{11} \mathrm{H}_{12}\right)$ structure $(\mathrm{M}=\mathrm{Li}$ or $\mathrm{Na})$ with orange $\mathrm{Li}^{+} / \mathrm{Na}^{+}$cation positions and large green $(\mathrm{B} / \mathrm{C}$ atoms) and gray ( $\mathrm{H}$ atoms) spheres denoting the diffraction-average spherical shells of scattering from the orientationally-disordered $\mathrm{CB}_{9} \mathrm{H}_{10}{ }^{-}$and $\mathrm{CB}_{11} \mathrm{H}_{12}{ }^{-}$anions (with arbitrary individual orientations superimposed). Adapted from [3].

$\mathrm{MCB}_{9} \mathrm{H}_{10}$ and $\mathrm{MCB}_{11} \mathrm{H}_{12}$ salts $[3,4]$. Neutron-elastic-scattering fixed-window scans (FWSs) on HFBS between $100 \mathrm{~K}$ and $400 \mathrm{~K}$ for the Li and Na sample mixtures are shown in Fig. 2. The onset of the drop in neutron elastic intensity near $240 \mathrm{~K}$ and $210 \mathrm{~K}$ for the Li and Na samples respectively indicates highly mobile anion reorientational motions approaching $10^{8}$ jumps $\mathrm{s}^{-1}$. The jump rates approach $10^{10}$ reorientational jumps s-1 near $330 \mathrm{~K}$ and $300 \mathrm{~K}$, respectively, as the FWS intensities level off again. For comparison, the much sharper intensity changes for the FWSs for the pure component salts at higher temperatures mark the more abrupt hysteretic phase-change behaviors from relatively immobile anions in the lower- $T$ ordered phases $\left(<10^{8}\right.$ jumps $\left.s^{-1}\right)$ to significantly more mobile anions in the high- $T$ disordered phases $\left(>10^{10}\right.$ jumps $\left.\mathrm{s}^{-1}\right)$. High anion mobilities ( $\geq 10^{10}$ jumps $/ \mathrm{s}$ above $300 \mathrm{~K}$ ) are a signature of closo-polyborate disordered phases and are believed to help facilitate rapid cation diffusive motions within the interstitial channels.

\footnotetext{
${ }^{1}$ NIST Center for Neutron Research, National Institute of Standards and Technology, Gaithersburg, MD 20899

2 University of Maryland, College Park, MD 20742

3 Tohoku University, Sendai 980-8577, Japan

${ }^{4}$ Ural Branch of the Russian Academy of Sciences, Ekaterinburg 620990, Russia

${ }^{5}$ National Renewable Energy Laboratory, Golden, C0 80401

${ }^{6}$ Sandia National Laboratories, Livermore, CA 94551
} 


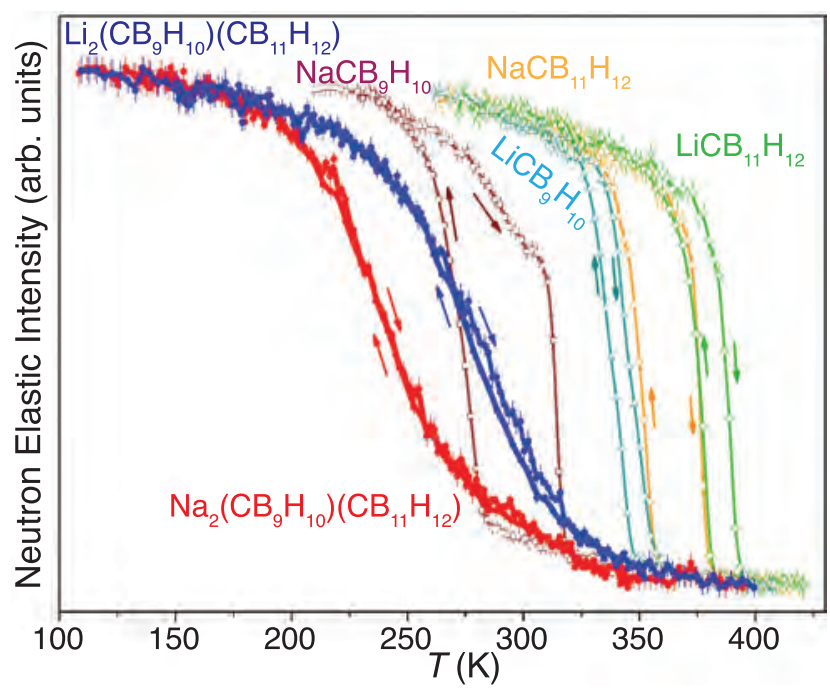

FIGURE 2: Neutron fixed-window scans for $\mathrm{M}_{2}\left(\mathrm{CB}_{9} \mathrm{H}_{10}\right)\left(\mathrm{CB}_{11} \mathrm{H}_{12}\right)(\mathrm{M}=\mathrm{Li}$ or $\mathrm{Na}$ ) compared with those for the various single-anion component compounds, using HFBS summed over all scattering angles covering a neutron momentum transfer $Q$ range of $0.87 \AA^{-1}$ to $1.68 \AA^{-1}$. Arrows differentiate heating and cooling scans. For a reasonable qualitative comparison, the individual data sets were scaled so as to have similar minimum and maximum intensities. Adapted from [3].

Proton spin-lattice relaxation rates from ${ }^{1} \mathrm{H}$ NMR measurements have confirmed the magnitudes and temperature-dependences of the anion orientational mobilities reflected by the FWSs. The activation energies for reorientational motion estimated from the NMR data are $220 \mathrm{meV}$ for $\mathrm{Li}_{2}\left(\mathrm{CB}_{9} \mathrm{H}_{10}\right)\left(\mathrm{CB}_{11} \mathrm{H}_{12}\right)$ and $180 \mathrm{meV}$ for $\mathrm{Na}_{2}\left(\mathrm{CB}_{9} \mathrm{H}_{10}\right)\left(\mathrm{CB}_{11} \mathrm{H}_{12}\right)$, values close to the activation energies found for the disordered phases of both $\mathrm{LiCB}_{11} \mathrm{H}_{12}$ and $\mathrm{NaCB}_{11} \mathrm{H}_{12}$ (177 meV).

Figure 3 shows the $T$-dependence of the ionic conductivities for $\mathrm{M}_{2}\left(\mathrm{CB}_{9} \mathrm{H}_{10}\right)\left(\mathrm{CB}_{11} \mathrm{H}_{12}\right)$ mixtures compared with those for the single-anion and other competing compounds [3]. The $\mathrm{Li}_{2}\left(\mathrm{CB}_{9} \mathrm{H}_{10}\right)\left(\mathrm{CB}_{11} \mathrm{H}_{12}\right)$ conductivity seems to match that for disordered $\mathrm{LiCB}_{9} \mathrm{H}_{10}$ and $\mathrm{Li}_{10} \mathrm{GeP}_{2} \mathrm{~S}_{12}$ above $350 \mathrm{~K}\left(\approx 0.04 \mathrm{~S} \mathrm{~cm}^{-1}\right)$, remaining somewhat lower than that for disordered $\mathrm{LiCB}_{11} \mathrm{H}_{12}$ above $380 \mathrm{~K}$. Below $350 \mathrm{~K}$, the $\mathrm{Li}_{2}\left(\mathrm{CB}_{9} \mathrm{H}_{10}\right)\left(\mathrm{CB}_{11} \mathrm{H}_{12}\right)$ conductivity decreases below that of a chief competitor $\mathrm{Li}_{10} \mathrm{GeP}_{2} \mathrm{~S}_{12}$ to $\approx 4 \mu \mathrm{S} \mathrm{cm}^{-1}$ by $243 \mathrm{~K}$. The conductivity in $\mathrm{Na}_{2}\left(\mathrm{CB}_{9} \mathrm{H}_{10}\right)\left(\mathrm{CB}_{11} \mathrm{H}_{12}\right)$ is much more impressive, seemingly even better than disordered $\mathrm{NaCB}_{9} \mathrm{H}_{10}$ and $\mathrm{NaCB}_{11} \mathrm{H}_{12}$ at all temperatures, and decreasing much less rapidly with temperature, from $\approx 0.07 \mathrm{~S} \mathrm{~cm}^{-1}$ at $300 \mathrm{~K}$ to $\approx 5 \mathrm{mS} \mathrm{cm}^{-1}$ at $243 \mathrm{~K}$. This disordered solid-solution $\mathrm{Na}^{+}$conductor displays substantially higher conductivity than any known solid $\mathrm{Na}^{+}$or $\mathrm{Li}^{+}$conductor.

Above room temperature, the conductivity barriers estimated from the slopes of $\ln (\sigma T)$ vs. $T^{-1}$ [311(2) meV and 226(2) meV for $\mathrm{Li}^{+}$and $\mathrm{Na}^{+}$, respectively], are in general agreement with those for the single-anion compounds [3, 4]. The overall barriers at sub-ambient temperatures are found to increase to 743(2) meV and 348(2) meV, respectively, signaling changes

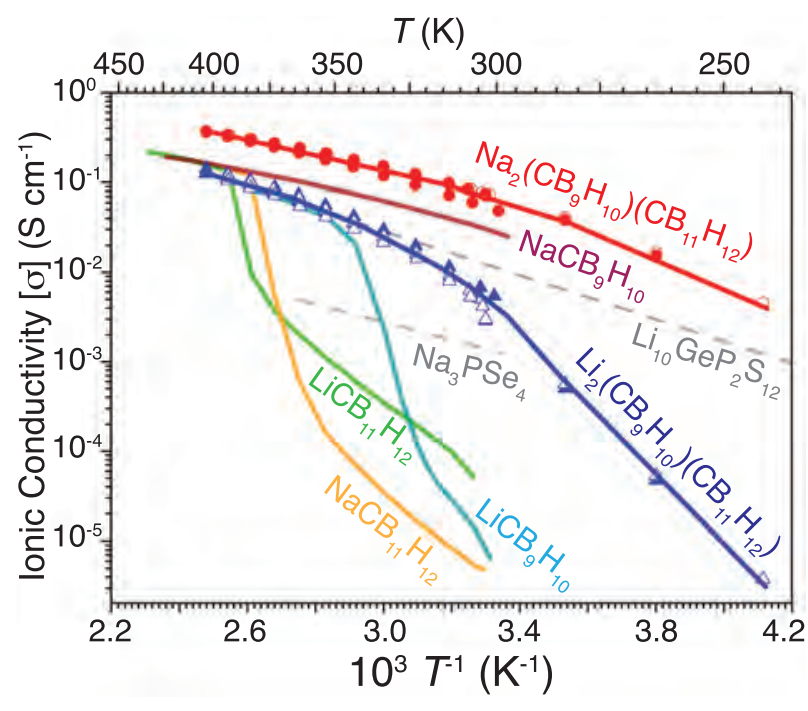

FIGURE 3: Temperature dependences of the ionic conductivities $(\sigma)$ for solution-dried, cold-pressed $\mathrm{Li}_{2}\left(\mathrm{CB}_{9} \mathrm{H}_{10}\right)\left(\mathrm{CB}_{11} \mathrm{H}_{12}\right)$ and $\mathrm{Na}_{2}\left(\mathrm{CB}_{9} \mathrm{H}_{10}\right)\left(\mathrm{CB}_{11} \mathrm{H}_{12}\right)$ using $L i$ and $A u$ electrodes, respectively, compared with those for the single-anion compounds and the closest-performing competitors, $\mathrm{Li}_{10} \mathrm{GeP}_{2} \mathrm{~S}_{12}$ and $\mathrm{Na}_{3} \mathrm{PSe}_{4}$ Adapted from [3].

in the rate-limiting steps. If the highly reorientationally mobile anions are enabling a reduced conductivity barrier at higher temperatures via a cooperative dynamical effect with the diffusing cations, the increase of the barrier with decreasing temperatures may indicate a reduced influence of the anions due to decreasing mobility. Comparison with the FWS results suggests that this cooperative dynamical effect may start to diminish for anion reorientational jump frequencies below roughly $10^{9} \mathrm{~s}^{-1}$. Further insights still await comprehensive molecular dynamics computations.

Mixing anions of different geometric "flavors" exemplifies an important advance in for further improving the remarkable conductive properties generally displayed by this class of materials, and represents a practical strategy for creating solid, superionic conductors for the variety of upcoming all-solid-state energy devices of the future.

\section{References}

[1] W. S. Tang, A. Unemoto, W. Zhou, V. Stavila, M. Matsuo, H. Wu, S. Orimo, T. J. Udovic, Energy Environ. Sci. 8, 3637 (2015).

[2] W. S. Tang, M. Matsuo, H. Wu, V. Stavila, W. Zhou, A. A. Talin, A. V. Soloninin, R. V. Skoryunov, O. A. Babanova, A. V. Skripov, A. Unemoto, S. Orimo, T. J. Udovic, Adv. Energy Mater. 6, 1502237 (2016).

[3] W. S. Tang, K. Yoshida, A. V. Soloninin, R. V. Skoryunov, O. A. Babanova, A. V. Skripov, M. Dimitrievska, V. Stavila, S. Orimo, T. J. Udovic, ACS Energy Lett. 1, 659 (2016).

[4] A. V. Soloninin, M. Dimitrievska, R. V. Skoryunov, O. A. Babanova, A. V. Skripov, W. S. Tang, V. Stavila, M. Matsuo, A. Unemoto, S. Orimo, and T. J. Udovic, J. Phys. Chem. C 121, 1000 (2017). 


\title{
Discovery of a dynamic odd-even effect in liquid n-alkanes near melting points
}

\author{
K. Yang, ${ }^{1,2}$ Z. Cai, ${ }^{3}$ A. Jaiswal, ${ }^{3}$ M. Tyagi, ${ }^{5,6}$ J. S. Moore, ${ }^{1,2,4}$ and Y. Zhang ${ }^{1,2,3}$
}

T he melting points of $n$-alkanes $\left(\mathrm{C}_{n} \mathrm{H}_{2 n+2}\right)$ as a function of the number of carbons $n$ show a zig-zag shape rather than a monotonic trend. This is probably the most well-known textbook example of the so-called "odd-even effect." Although this striking odd-even effect was known more than a century ago, its molecular origin was only revealed in the last two decades. In short, single-crystal diffraction confirmed that evennumbered $n$-alkanes pack more efficiently into ordered periodic crystalline structures than do the odd-numbered $\mathrm{n}$-alkanes, thus, they exhibit higher densities and melt at higher temperatures [1]. Therefore, such an odd-even effect is not expected in the liquid state because of the lack of long range order, as assumed by the classical Kauzmann-Eyring theory of molecular viscous flow [2]. In this study, we discovered a surprising dynamic odd-even effect in the translational diffusion of $\mathrm{n}$-alkanes in their liquid states [3]. We utilized the disk chopper time-of-flight spectrometer (DCS) at NIST Center for Neutron Research (NCNR) to measure the incoherent quasi-elastic neutron scattering (QENS) spectra of liquid $n$-alkanes. Our results suggest that the dynamic properties of $n$-alkanes are extremely sensitive to the number of carbons even though n-alkanes are the simplest hydrocarbon molecules. The transport properties of liquid n-alkanes - the principle components of crude oil and gasoline - are fundamental to petroleum science and engineering and of central importance to a wide spectrum of technologically-important chemical processes, such as lubrication, diffusion through porous media, and heat transfer.

The high-resolution incoherent QENS benefits from the exceptionally large incoherent cross section of hydrogen, and thus it is ideally suited to probe the single particle motions, such as hydrogen diffusion with picosecond resolution. Various dynamic processes, spanning from fast segmental relaxations to relatively slower translational and rotational diffusion, exist in n-alkanes. In comparison to bulk properties, the microscopic dynamics directly reflects the subtle differences between individual $\mathrm{n}$-alkanes in the series.

The QENS spectra measured using DCS are shown in Figure 1. We chose the incident neutron wavelength to be $8 \AA$, which provided an elastic energy resolution of approximately $30 \mu \mathrm{eV}$

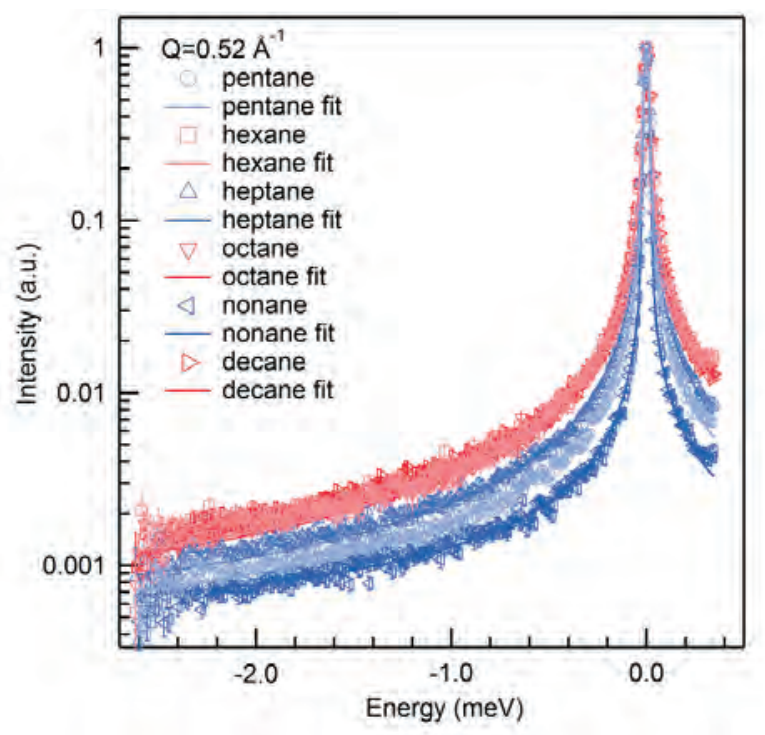

FIGURE 1: Incoherent Quasi-Elastic Neutron Scattering (QENS) spectra clearly show an odd(red)-even(blue) alternation of the relaxational dynamics of n-alkanes (n= $5-10$ ) at a wave vector transfer $Q=0.52 \AA^{-1}$.

full width at half maximum (FWHM). We measured the QENS spectra slightly ( $3 \mathrm{~K})$ above each $\mathrm{n}$-alkane's melting point $\left(T_{m}+3 \mathrm{~K}\right)$. This temperature set was chosen to decouple the melting temperature odd-even effect from the dynamic properties and to ensure that the $n$-alkanes were in the liquid state throughout the data acquisition process (about $6 \mathrm{~h}$ per sample). For comparison, we also measured the QENS spectra for all $\mathrm{n}$-alkanes samples at a constant temperature of $246 \mathrm{~K}$. This temperature $(246 \mathrm{~K})$ corresponds to the highest temperature (the melting point of decane plus $3 \mathrm{~K}$ ) used in the first set of temperatures.

Incoherent QENS measures the self-dynamic structure factor, which is the Fourier transform of the intermediate scattering function $F_{\mathrm{s}}(Q, t)$ that quantifies the single-particle correlations of the system. $F_{\mathrm{s}}(Q, t)$ can be decomposed into the product of the translational correlation function $F_{\mathrm{T}}(Q, t)$ and the rotational correlation function $F_{\mathrm{R}}(Q, t)$ of the hydrogens of the cations. 
For a glass-forming liquid, $F_{\mathrm{T}}(Q, t)$ can be described by the stretched exponential (KWW) function:

$$
F_{\mathrm{T}}(Q, t)=\exp \left[-\left(\frac{t}{\tau_{\mathrm{T}}}\right)^{\beta}\right],
$$

where $\tau_{\mathrm{T}}$ is the relaxation time and $\beta$ is the stretching exponent.

The $Q$ and $t$ dependence of $F_{\mathrm{R}}(Q, t)$ can be expressed by the Sears expansion. Here, we terminate the expansion at the first three terms because the higher-order terms are negligible in our experimental $Q$ range. Then, the expression for $F_{\mathrm{R}}(Q, t)$ is:

$$
F_{\mathrm{R}}(Q, t)=j_{0}^{2}(Q a)+3 j_{1}^{2}(Q a) \exp \left[-\frac{t}{3 \tau_{\mathrm{R}}}\right]+5 j_{2}^{2}(Q a) \exp \left[-\frac{t}{\tau_{\mathrm{R}}}\right],
$$

where $a$ stands for the radius of the rotation, $\tau_{\mathrm{R}}$ is the relaxation time associated with the rotational diffusion, and $j_{\mathrm{n}}(x)$ are the spherical Bessel functions. The measured spectra can be described remarkably well with the translational contribution alone at low $Q$. Only at larger $Q$ values is the addition of the rotational contribution needed to improve the fitting. Demonstrations of the fittings of the QENS spectra for all samples at one $Q$ are illustrated in Figure 1 .

The fitted inverse relaxation time $1 / \tau$ and stretching exponent $\beta$ are plotted as functions of $Q^{2}$ and $Q$ respectively (Figure 2a, 2b) for $Q=0.52 \AA^{-1}$. The relaxation time, $\tau$, for translational diffusion is in the order of $10 \mathrm{ps}$. All even-numbered n-alkanes exhibit smaller values of $\tau$ compared to those for odd-numbered alkanes. This is easily identified by the clear separation between the blue curves (odd-numbered n-alkanes) and the red curves (even-numbered n-alkanes) in Figure 2a. A consistent trend was observed in the plot of $\beta$ versus $Q$ (Figure $2 \mathrm{~b}$ ): even-numbered n-alkanes exhibit similar values of $\beta$ at different $Q$ while odd-numbered alkanes exhibit smaller values of $\beta$.

Plotting the extracted relaxation time $\tau$ and stretching exponent $\beta$ as a function of carbon number $\mathrm{n}$ for all samples at three representative $Q$ values (Figure $2 c, 2 d$ ) shows a very clear odd-even effect in the dynamics of $n$-alkanes. Given the fact that all data were acquired in the liquid state near the melting point, this is the first time, to the best of our knowledge, that a dynamic odd-even effect at the molecular level has been observed for $\mathrm{n}$-alkanes. The odd-numbered n-alkanes have longer relaxation times than do their neighboring even-numbered species, which means that pentane, heptane, and nonane move more slowly than do hexane, octane, and decane. It is very interesting that all even-numbered n-alkanes have similar relaxation times (e.g., about 3 ps at $0.97 \AA^{-1}$ ) near their melting point while the odd-numbered n-alkanes' relaxation times vary across the $Q$-range.

Unlike the density and viscosity, the amplitude of this dynamic odd-even effect goes beyond the simple odd-even trend of the $n$-alkanes melting points. Taking hexane and heptane, for example, the difference between their melting points is only $4 \mathrm{~K}$ while their relaxation times differ by more than a factor of 2 . The extreme case is nonane, which exhibits much slower dynamics than does octane. Despite the fact that the melting points differ by only $3 \mathrm{~K}$, the dynamics of nonane are more than 30 times slower than those of octane at $0.52 \AA^{-1}$. This result is extremely surprising given that the structural difference between nonane and octane is only one methylene group.
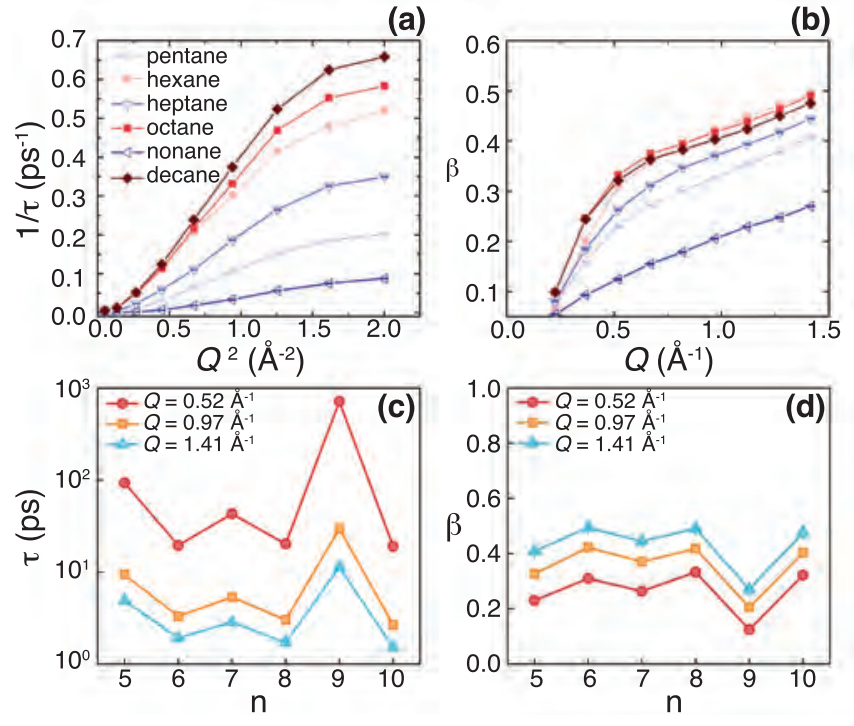

FIGURE 2: $Q$-dependence of the fitting parameters: inverse relaxation time $1 / \tau$ vs $Q^{2}$ (a) and stretching exponent $\beta$ (b) vs $Q$ of $\mathrm{n}$-alkanes near their respective melting points. Odd-numbered n-alkanes are plotted in blue and even-numbered $\mathrm{n}$-alkanes are plotted in red. Both clearly show and odd-even alternation as the blue and red curves are separate; the n-dependence of the extracted relaxation time $\tau$ (c) and stretching exponent $\beta$ (d) of liquid $n$-alkanes near their respective melting points clearly shows a dynamic odd-even effect. Data are shown for three representative $Q$ values.

In summary, with high resolution QENS measurements, we have discovered a surprising dynamic odd-even effect in liquid n-alkanes near their melting points. This observation firmly establishes that an odd-even effect does exist in the liquid state and is more prominent in the dynamic properties than in the thermodynamic quantities [3-5]. Furthermore, it suggests that mechanisms other than periodic packing should be scrutinized in order to provide a more thorough understanding of the odd-even effect. We hypothesize that the extra $\mathrm{CH}_{2}$ group switches the structural symmetry of neighboring n-alkanes and thus affects the configurational entropy of the liquids, which fundamentally leads to the alternating trend of the transport properties. Another plausible scenario is that the local packing structures of the $n$-alkane molecules are different, albeit too small to detect conveniently, between the odd and even species. In any case, the exact mechanism responsible for this dynamic odd-even effect of liquid n-alkanes requires high precision simulations and careful re-examinations and extensions of the classical theories of molecular viscous flow.

\section{References}

[1] R. Boese, H. Weiss, D. Blaser, Angew. Chem. Int. Ed. 38, 988 (1999).

[2] W. Kauzmann, H. Eyring, J. Am. Chem. Soc. 62, 3113 (1940).

[3] K. Yang, Z. Cai, A. Jaiswal, M. Tyagi, J. S. Moore, Y. Zhang, Angew. Chemie Int. Ed. 55, 14090 (2016).

[4] K. Yang, Z. Cai, M. Tyagi, M. Feygenson, J. C. Neuefeind, J. S. Moore, Y. Zhang, Chem. Mater. 28, 3227 (2016).

[5] K. Yang, M. Tyagi, J. S. Moore, Y. Zhang, J. Am. Chem. Soc. 136, 1268 (2014). 


\title{
New metastable materials help answer what makes iron so special for superconductivity
}

\author{
X. Zhou, ${ }^{1,2}$ B. Wilfong, ${ }^{1,2}$ H. Vivanco, 1 J. Paglione, ${ }^{2,3}$ C. Brown, ${ }^{4}$ and E. E. Rodriguez ${ }^{1,2}$
}

$\mathrm{T}$ he simple binary compound, iron selenide (FeSe), was discovered to be a superconductor in 2008 with a critical temperature $\left(T_{\mathrm{c}}\right)$ of $8 \mathrm{~K}$ [1]. Since then, more extraordinary claims have been made with respect to its superconducting properties including increasing $T_{\mathrm{c}}$ to $37 \mathrm{~K}$ upon applying pressure [2], $42 \mathrm{~K}$ upon inserting other chemical species [3], and $65 \mathrm{~K}$ upon isolating it as a single layer [4]. But why does this material require iron for its superconductivity? That remains an outstanding question in the field of unconventional superconductivity. We have sought to answer this question by preparing cobalt analogues of FeSe and FeS and studying them with neutron diffraction. We prepared for the first time tetragonal CoSe and CoS, which are metastable materials, and studied their physical properties including magnetization and electrical resistivity. Our results [5] indicate that the electron configurations of the $\mathrm{Co}$ and $\mathrm{Fe}$ atoms provide clues as to what makes iron so special for superconductivity.

To prepare the Co analogue of the superconductor FeSe, we utilized what is known as chemie douce, or soft chemistry, methods. While FeSe can be prepared by more conventional means such as solid-state reactions, CoSe cannot be synthesized by similar methods. FeSe is a thermodynamic product of a high temperature reaction of iron with selenium, but CoSe with the same crystal structure can only be isolated as a kinetic product at low temperature and is therefore metastable. A solution to this synthetic challenge was to first prepare the thermodynamically stable $\mathrm{KCO}_{2} \mathrm{Se}_{2}$, which has a similar structure to $\mathrm{CoSe}$, and remove the $\mathrm{K}^{+}$cations post-synthetically in aqueous media. Figure 1 demonstrates the basic structural unit common to $\mathrm{KCO}_{2} \mathrm{Se}_{2}$ and CoSe and how we can manipulate them via our chemie douce methods to achieve the desired products. The same chemistry was extended to the sulfides to prepare metastable CoS.

Once we kinetically stabilized and isolated phase-pure CoSe, we measured its magnetization and electrical resistivity to compare how this new compound is similar to superconducting FeSe. From resistivity measurements, we found CoSe to be metallic, similar to FeSe, from room temperature down to low temperatures, but no superconducting transition was

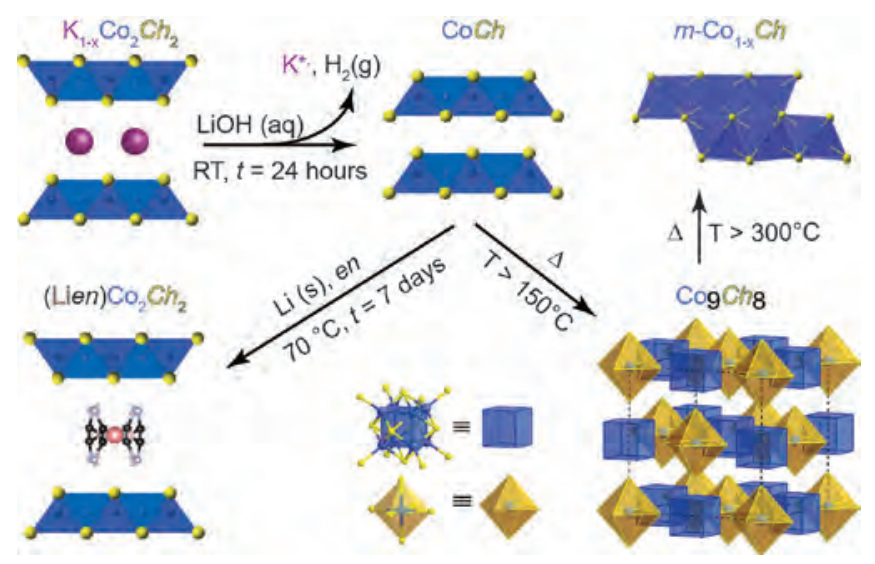

FIGURE 1: Routes towards preparing various cobalt chalcogenides (CoCh) through soft chemistry. The new metastable CoSe is the first transition metal analogue of superconducting FeSe.

observed down to $2 \mathrm{~K}$. Magnetization measurements showed that instead of a Meissner effect at low temperatures, both CoSe and CoS displayed a divergence in the magnetization at approximately $10 \mathrm{~K}$. Furthermore, at $2 \mathrm{~K}$ CoSe displayed some hysteresis in its magnetization as a function of applied magnetic field. Therefore, it seems plausible that CoSe may be a weak ferromagnetic (or ferrimagnetic) metal at low temperatures instead of a superconductor.

To help better answer why CoSe does not display superconductivity as in closely related FeSe, we performed powder neutron diffraction (PND) experiments at the BT-1 high-resolution diffractometer. Since neutrons afford information on both crystal structure and magnetic ordering, we were able to obtain a highly accurate structure to compare against FeSe and understand CoSe's magnetization properties. Figure 2 shows the structural fit to the PND data at low temperatures including a magnetic moment on the Co site $\left(\approx 0.30 \mu_{\mathrm{B}}\right)$. The observed small moment of $\mathrm{Co}$ is in line with the magnetization measurements, which show a converging but unsaturated moment of less than $0.1 \mu_{\mathrm{B}}$ with applied magnetic field up to $7 \mathrm{~T}$. Furthermore, we were able to demonstrate that CoSe remains tetragonal (P4/nmm space group) down to base 


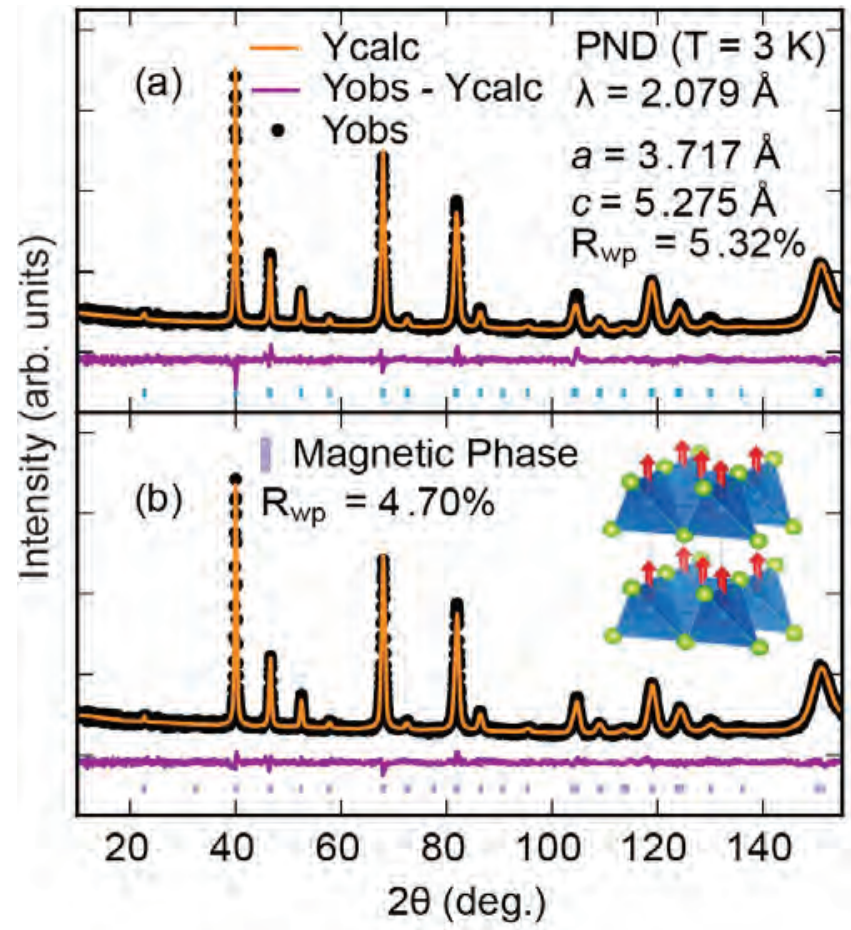

FIGURE 2: Rietveld refinement of PND data of CoSe a) without and b) with considering the magnetic contribution. The magnetic moment is aligned along the $c$-axis as illustrated in the inset of $b$ ).

temperature. This is already a significant change from FeSe, which undergoes a tetragonal to orthorhombic distortion near $90 \mathrm{~K}[6]$.

With CoSe's crystal structure obtained from the high-resolution neutron data, we performed density functional theory (DFT) calculations to determine the electronic structure of CoSe. Even though one cannot predict superconductivity from such electronic band structures, we could compare some of the features between CoSe and FeSe to learn how their differences lead to the observed physical properties. Figure 3 compares the electron dispersion curves along particular high symmetry directions in CoSe and FeSe and shows the corresponding electronic density of states (DOS). In these selenides, Fe(II) is has a $d^{6}$ electron configuration while that for $\mathrm{Co}(\mathrm{II})$ is $\mathrm{d}^{7}$, and this leads to the Fermi level being higher in energy in CoSe with respect to FeSe. In CoSe, the Fermi level lies on a peak on the DOS, which helps to explain why CoSe can be classified as an itinerant weak ferromagnet. For FeSe, however, the Fermi level lies in a pseudogap of the DOS, which helps to explain why it is not a ferromagnet. This occupation of the psuedogap in FeSe is also partly responsible for its thermodynamic stability in the tetragonal structure, unlike CoSe, which is metastable in this structure. Furthermore, the Fermi level in FeSe occupies the so-called electron pockets at the M-point, which may hold the key for superconductivity in the iron-based materials. Our DFT calculations show that CoSe does not contain such electron pockets.
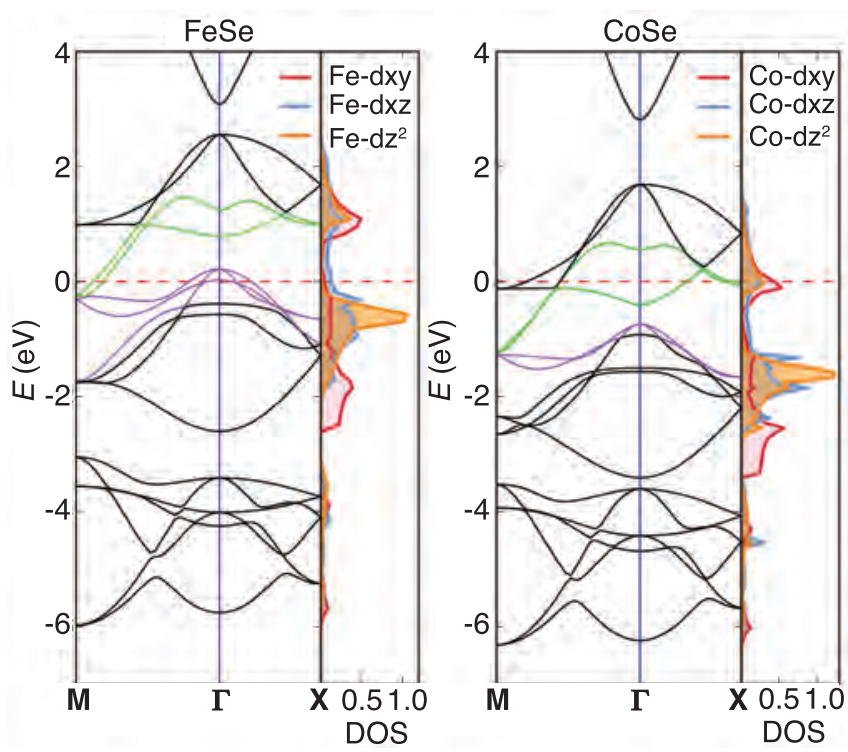

FIGURE 3: Band structure along three different directions and the corresponding partial DOS of $3 d$ states for FeSe and CoSe. Purple and green lines in the band structure indicate bands crossing the Fermi level close to the $\Gamma$ and $\mathrm{M}$ points, respectively.

In summary, our work on CoSe and FeSe demonstrates the importance of using novel chemical means to prepare metastable materials. Our synthesis helped us obtain, for the first time, an analogue to the FeSe superconductor in order to study what makes iron so special for this extraordinary superconductivity. The physical property measurements demonstrated the vastly different properties that can arise from substituting Co for Fe in this structure. With neutron diffraction, we not only obtained accurate crystallographic information, but we also determined the nature of the observed magnetic ordering in CoSe. Finally, through our DFT studies we demonstrated that the common square lattice in CoSe and FeSe leads to electronic structures easy to interpret that may even give us a tool by which to predictably tune the physical properties of these selenides. From this work, we hope to prepare other transition metal analogues to FeSe and better understand how to design new magnetic materials and superconductors.

\section{References}

[1] F.-C. Hsu et al., P. Natl. Acad. Sci. USA 105, 14262 (2008).

[2] T. Imai, K. Ahilan, F. L. Ning, T. M. McQueen, R. J. Cava, Phys. Rev. Lett. 102, 177005 (2009).

[3] X. Zhou, C. K. H. Borg, J. W. Lynn, S. R. Saha, J. Paglione, E. E. Rodriguez, J. Mater. Chem. C 4, 3934 (2016).

[4] S. He et al., Nat. Mater. 12, 605 (2013).

[5] X. Zhou, B. Wilfong, H. K. Vivanco, J. Paglione, C. M. Brown, E. E. Rodriguez, J. Am. Chem. Soc. 138, 16432 (2016).

[6] T. M. McQueen, A. J. Williams, P. W. Stephens, T. J., Y. Zhu, V. Ksenofontov, F. Casper, C. Felser, R. J. Cava, Phys. Rev. Lett. 103, 057002 (2009). 


\section{Long-lived Higgs amplitude mode in a two-dimensional quantum antiferromagnet near the quantum critical point}

T. Hong, ${ }^{1}$ M. Matsumoto, ${ }^{2}$ Y. Qiu, ${ }^{3}$ W. Chen, ${ }^{3,4}$ T. R. Gentile, ${ }^{3}$ S. Watson, ${ }^{3}$ F. F. Awwadi, ${ }^{5}$ M. M. Turnbull, ${ }^{6}$

S. E. Dissanayake, ${ }^{1}$ H. Agrawal,,$^{7}$ R. Toft-Petersen, ${ }^{8}$ B. Klemke, ${ }^{8}$ K. Coester, ${ }^{9}$ K. P. Schmidt, ${ }^{10}$ and D. A. Tennant ${ }^{1}$

T he concept of mass generation by means of the Higgs mechanism is of crucial importance to particle physics. In the Standard Model, the excitation of the amplitude fluctuation of the condensed Higgs field manifests as the massive Higgs boson [1]. Since its discovery, there has been much interest in searching for Higgs boson-like quasiparticles in condensed matter physics [2]. The collective excitations in the broken-symmetry phase near a quantum critical point (QCP) can be characterized by fluctuations of the phase and amplitude of the order parameter. The phase oscillations correspond to the Nambu-Goldstone modes where the massive amplitude mode, analogous to the Higgs boson in particle physics, is prone to decay into a pair of low-energy Nambu-Goldstone modes in low dimensions. Especially, observation of the Higgs amplitude mode in two dimensions is an outstanding experimental challenge.

The quantum $S=1 / 2$ Heisenberg antiferromagnetic two-leg spin ladder is one of the cornerstone models in low-dimensional quantum magnetism. In the one-dimensional limit of isolated spin-1/2 ladders, the ground state consists of valence-bond singlets on each rung of the ladder. Interestingly, the ground state can be tuned by the inter-ladder coupling from the quantum disordered (QD) state, through the QCP, to the long-range magneticallyordered (LRO) state as shown in Fig. 1(a). In the QD phase, the magnetic excitations are triply-degenerate magnons with a spin gap energy that vanishes on approach to the QCP. In the LRO phase, the triplet modes evolve into two Nambu-Goldstone or transverse modes (TMs), reflecting spin fluctuations perpendicular to the ordered moment, accompanied by a longitudinal mode (LM), reflecting spin fluctuations along the ordered moment. The latter mode is analogous to the Higgs amplitude mode.

\section{It has been shown that the metal-organic compound} $\mathrm{C}_{9} \mathrm{D}_{18} \mathrm{~N}_{2} \mathrm{CuBr}_{4}$ (DLCB for short) is a unique spin ladder material where the inter-ladder coupling is sufficiently strong to drive the system into the LRO phase [3]. Figure 1(b) shows the molecular two-leg ladder structure of DLCB. A minimal two-dimensional (2D) spin interacting model was proposed based on the crystal structure. Indeed, the inter-layer dispersion is absent within the instrumental resolution, and the observed magnon dispersions can be described by this model quantitatively. A spin gap energy

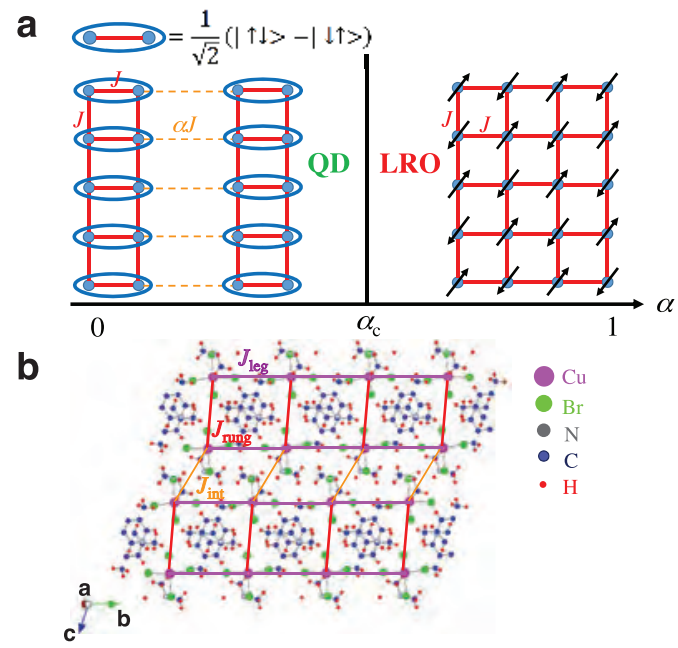

FIGURE 1: (a) Schematic diagram of coupled two-leg square spin ladders, where the ground state can be tuned by the interladder coupling from the quantum disordered (QD) state, through a quantum critical point in two dimensions, to the long-range magnetically ordered (LR0) state. Blue circles stand for the spin-1/2 magnetic ions. The ellipses represent a singlet valence bond of spins. (b) The molecular two-leg ladder structure with the leg direction along the $b$-axis and a two-dimensional model for the magnetic interactions in DLCB.

$\Delta=0.32(3) \mathrm{meV}$ results from a small Ising anisotropy. Importantly, the analysis of the spin Hamiltonian indicates that DLCB is close to the QCP, making it an ideal candidate to investigate the Higgs amplitude mode.

Since TMs and the LM/Higgs amplitude mode in DLCB could be degenerate or unresolvable within the instrumental resolution, we employed polarized neutron scattering on the cold neutron spectrometer MACS to unambiguously determine the nature of spin polarization of magnetic excitation spectra [4]. The polarization analysis was performed using the recently developed capability of wide-angle ${ }^{3} \mathrm{He}$ spin filters. Figure $2(\mathrm{a})$ shows a rocking scan of the nuclear Bragg reflection $(0,1,-1)$ using the non-spin-flip (NSF) and spin-flip (SF) configurations, respectively. The calculated flipping ratio is as high as $I_{\mathrm{NSF}} / I_{\mathrm{SF}}=43(1)$. Figure 2 (b) shows a rocking scan of the magnetic Bragg reflection $(0.5,0.5,-0.5)$ at $T=1.4 \mathrm{~K}$ using NSF and SF configurations, respectively. The NSF scattering intensity

\footnotetext{
1 Quantum Condensed Matter Division, Oak Ridge National Laboratory, Oak Ridge, TN 37831

2 Shizuoka University, Shizuoka 422-8529, Japan

${ }^{3}$ NIST Center for Neutron Research, National Institute of Standards and Technology, Gaithersburg, MD 20899

${ }^{4}$ University of Maryland, College Park, MD 20742

${ }^{5}$ The University of Jordan, Amman 11942, Jordan
}

\footnotetext{
${ }^{6}$ Clark University, Worcester, MA 01610

7 Instrument and Source Division, Oak Ridge National Laboratory, Oak Ridge, TN 37831

${ }^{8}$ Helmholtz-Zentrum Berlin für Materialien und Energie, D-14109 Berlin, Germany

${ }^{9}$ Lehrstuhl für Theoretische Physik I, TU Dortmund, D-44221 Dortmund, Germany

${ }^{10}$ Lehrstuhl für Theoretische Physik I, Staudtstrasse 7, Universität Erlangen-

Nürnberg, D-91058, Germany
} 


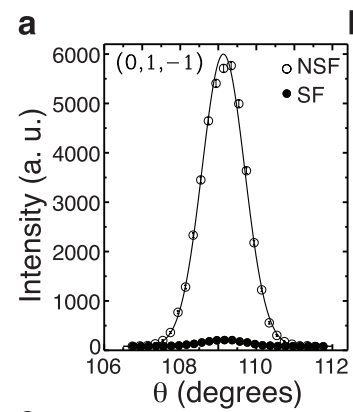

b
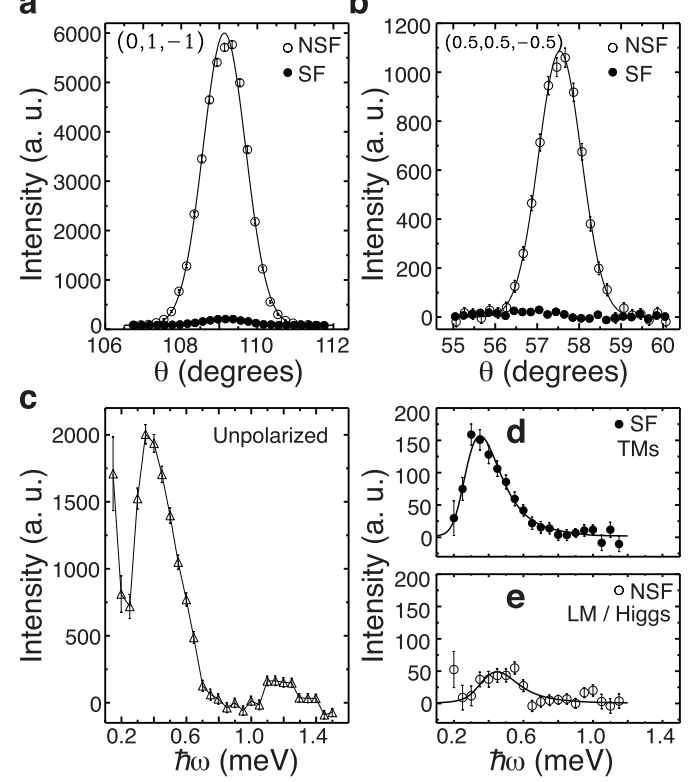

FIGURE 2: Background-subtracted rocking scans at (a) the nuclear Bragg reflection $(0,1,-1)$ and $(b)$ the magnetic Bragg reflection $(0.5,0.5,-0.5)$ using NSF and SF configurations, respectively. Solid lines are fits to a Gaussian function. Background-subtracted energy scans at $Q=(0.5,0.5,-0.5)$ using (c) unpolarized neutron, (d) SF and (e) NSF configurations, respectively. Solid lines are fits to a two-Lorentzian damped harmonic-oscillator model convolved with the instrumental resolution function. All experimental data were collected at $T=1.4 \mathrm{~K}$. Error bars represent one standard deviation.

dominates the SF intensity, which suggests that the out-of-plane spin component is dominant, thus confirming the orientation of the staggered moments.

The principles for polarized neutron scattering can be summarized as follows: a) phonons and structural scattering are seen in the NSF channel; b) components of spin fluctuations parallel to the neutron polarization are seen in the NSF channel; c) components of spin fluctuations perpendicular to the neutron polarization are seen in the SF channel. The polarized neutron experiment was designed so that TMs and the LM would appear separately in the SF and NSF configurations, respectively. For purposes of comparison, Fig. 2(c) shows an energy scan at $Q=(0.5,0.5,-0.5)$ using unpolarized neutrons. Figures 2 (d) and (e) show the same energy scans in the SF and NSF channels. The fitted spin gap energies of the TMs and LM/Higgs amplitude mode were $\Delta_{\mathrm{TMs}}=0.33(3) \mathrm{meV}$ and $\Delta_{\mathrm{LM}}=0.48(3) \mathrm{meV}$, respectively. Moreover, we managed to map out the excitation spectra in the Brillouin zone. Figure 3 summarizes a comparison between experimental data and theoretical calculations of the spin-wave spectra along two high-symmetry directions in reciprocal space. In the SF configuration, the observed magnetic excitations in Figs. 3(c) and (d) are in excellent agreement with the calculation using linear spin-wave theory (LSWT) and thus were confirmed to be TMs.

Since the Higgs amplitude mode is not predicted by LSWT, we used bond-operator theory (BOT) to describe the low-energy excitations near the QCP in DLCB. Figures $3(\mathrm{e})$ and (f) show the calculated excitation spectra using BOT, which reproduce the experimental data qualitatively. Thus, our conclusion that the nature of the spin excitations observed in the NSF configuration is due to spin fluctuations along the staggered moment direction

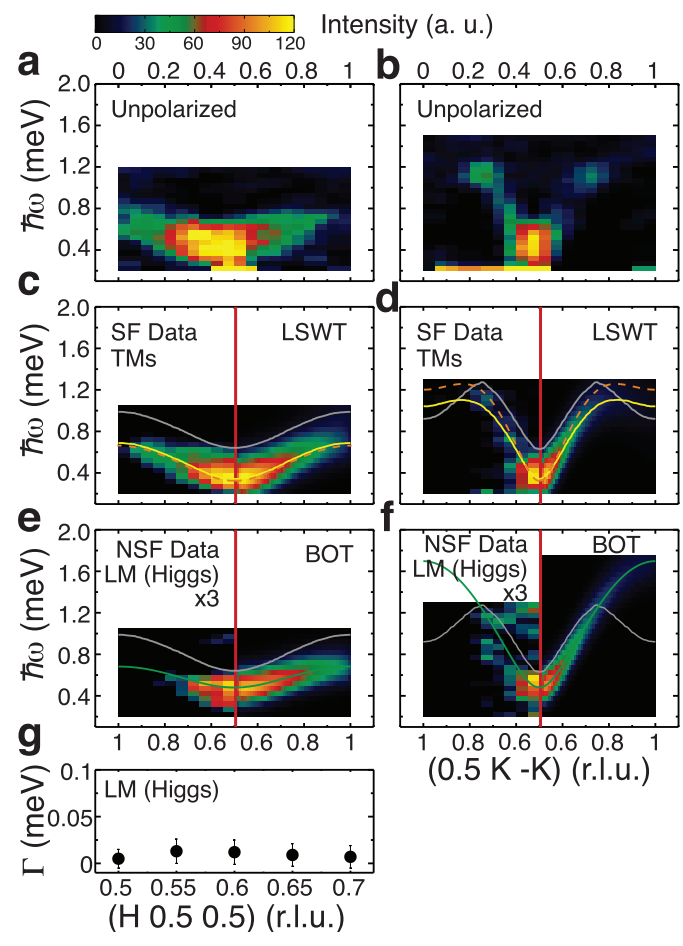

a

FIGURE 3: (a-b) False-color maps of the background-subtracted magnetic excitation spectra as a function of energy and wavevector transfer measured using unpolarized neutrons. (c-d) Comparison between experimental data using the spin-flip (SF) configuration and a calculation using linear spin-wave theory (LSWT). (e-f) Comparison between experimental data using the non-spin-flip (NSF) configuration and a calculation using bond-operator theory (BOT) along two high- symmetry directions in reciprocal space, respectively. Intensities have been enhanced by a factor of 3 . Dashed brown lines are the calculation using high-order series expansions. Solid yellow lines are the transverse modes (TMs) calculated using LSWT. Solid green lines are the longitudinal mode (LM)/Higgs amplitude mode calculated using BOT. Solid grey lines are the calculated lower-boundary of the two-magnon continuum. $(\mathrm{g})$ The wavevector dependence of the resolutioncorrected intrinsic linewidth of the Higgs amplitude mode. All experimental data were collected at $T=1.4 \mathrm{~K}$.

is quite convincing. Moreover, the grey lines in Figs. 3(e) and (f) are the calculated lower boundary of the two-magnon continuum in DLCB, which lies well above the Higgs amplitude mode. Hence, the spontaneous decay of the Higgs amplitude mode into a pair of TMs is forbidden by energy and momentum conservation.

In summary, the unique ability of polarized neutron scattering on MACS to probe the spin polarization of dynamic spin paircorrelation functions allows us to distinguish the Higgs amplitude mode from the dominant transverse Nambu-Goldstone mode in a two-dimensional $S=1 / 2$ antiferromagnet DLCB. The opening of an anisotropic energy gap kinematically prevents the decay of the Higgs amplitude mode into a pair of transverse modes. This leads to the long lifetime for the Higgs amplitude mode and makes it observable near the quantum critical point in two dimensions.

\section{References}

[1] P. W. Higgs, Phys. Rev. Lett. 13, 508 (1964).

[2] D. Pekker, C. M. Varma, Ann. Rev. Condens. Matter Phys. 6, 269 (2015).

[3] T. Hong et al., Phys. Rev. B 89, 174432 (2014).

[4] T. Hong et al., Nat. Phys. 13, 638 (2017). 


\title{
Complex three-dimensional magnetic ordering in segmented nanowire arrays
}

\author{
A. J. Grutter, ${ }^{1}$ K. L. Krycka, ${ }^{1}$ E. V. Tartakovskaya,${ }^{2,3}$ J. A. Borchers, ${ }^{1}$ K. Sai Madhukar Reddy, ${ }^{4}$ \\ E. Ortega, ${ }^{5}$ A. Ponce, ${ }^{5}$ and B. J. H. Stadler ${ }^{4}$
}

$\mathrm{H}$ igh density arrays of ferromagnetic nanowires are critical to realizing next-generation 3D spintronic technologies $[1,2]$ In particular, segmented nanowires have recently emerged as exciting routes towards magnetic device architectures such as racetrack memory [3]. To attain the high information densities that are desired, nanowires within the array must be in close proximity. However, the effect of long-range interactions in closely-packed nanowires has long remained untested due to the challenges of developing a 3D picture of the complex magnetic ordering that can emerge. Individual segmented nanowires can readily be studied through magnetic imaging or scanning probe techniques, but the array is usually embedded in an alumina oxide $\left(\mathrm{Al}_{2} \mathrm{O}_{3}\right)$ template to maintain order and alignment between nanowires. Thus, the interwire coupling is inaccessible unless the array is destroyed to allow common probes to be employed. Further, most theoretical treatments are based on non-interacting wires or continuum approximations - two assumptions which are not appropriate for closely packed segmented nanowires. In reality, the combination of intersegment and interwire coupling in these systems may result in long-range magnetic correlations.

To address these challenges, polarization analyzed small angle neutron scattering (PASANS) was used to probe hexagonal arrays of nanowires comprised of alternating segments of ferromagnetic galfenol $\left(\mathrm{Fe}_{0.8} \mathrm{Ga}_{0.2}\right)$ and non-magnetic $\mathrm{Cu}$ with $35 \mathrm{~nm}$ diameter, $100 \mathrm{~nm}$ wire separation, and $\mathrm{FeGa} / \mathrm{Cu}$ thicknesses of $12 \mathrm{~nm} / 5 \mathrm{~nm}$ [4]. PASANS is a unique capability that can be applied to the analysis of the structural and magnetic order of the nanowire array in 3D. In PASANS, a beam of neutrons is incident on the sample in the $Z$-direction (Figure 1a). Figure $1 \mathrm{~b}$ shows a typical two-dimensional scattering pattern for our system, captured as a function of the wavevector $Q$ on an area sensitive detector. Four different scattering cross sections were measured by selecting the orientation of the neutron spin ( $\uparrow$ or $\downarrow$ ) before and after scattering from the sample with a supermirror polarizer and ${ }^{3}$ He spin filter, respectively. The non-spin-flip (NSF) scattering $(\uparrow \uparrow)$ and $(\downarrow \downarrow)$, contains information about both the physical structure and the magnetic moments parallel to an applied magnetic field. As a complement, the two spin-flip (SF) cross sections, $(\uparrow \downarrow)$ and $(\downarrow \uparrow)$, probe magnetic ordering perpendicular
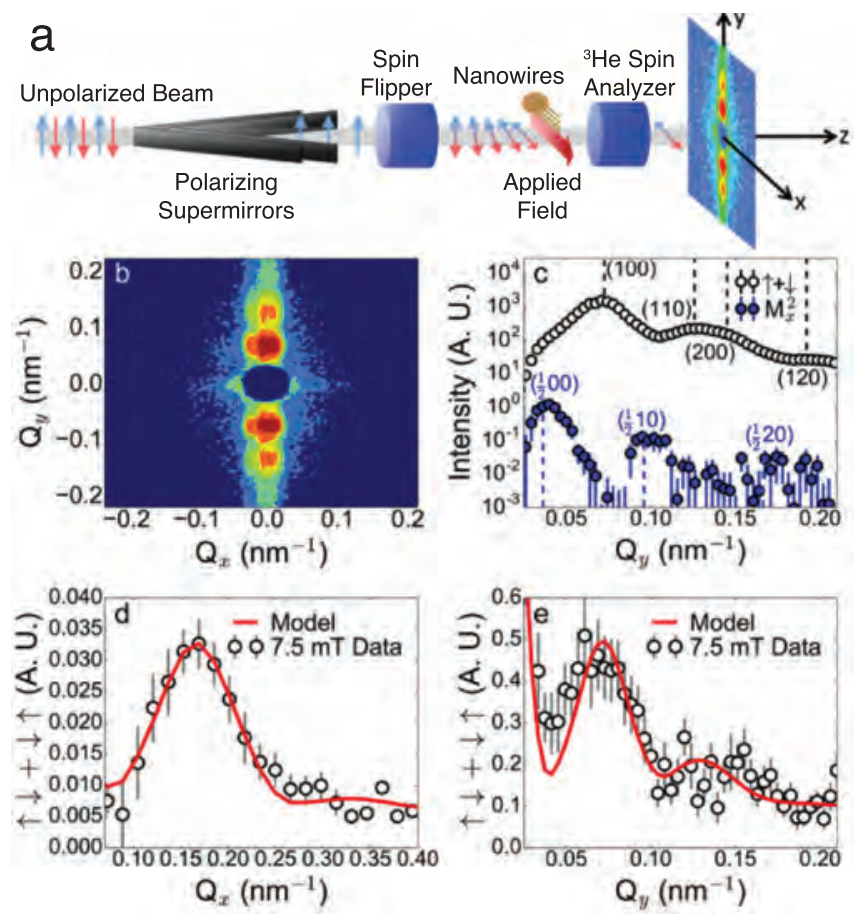

FIGURE 1: (a) Schematic of the beamline configuration for the PASANS measurements. (b) Typical unpolarized 2-D SANS pattern produced by our (Fe Ga 12/Cu 5.0) ${ }_{350} \mathrm{~nm}$ sample with the geometry shown in part (a). (c) Sum of the up and down intensities is proportional to $\left.\operatorname{IN}\left(Q_{y}\right)\right|^{2}$ shown in black. Normalized $\left|\operatorname{Mx}\left(Q_{y}\right)\right|^{2}$ is obtained from the difference (blue) of the up and down intensities for a cut through the scattering along $Q_{\mathrm{y}}$ (interwire direction) at $700 \mathrm{mT}$. Black and blue dashed lines show the theoretical peak locations for the structural and magnetic peaks, respectively. (d) Spin-flip scattering cut along the $Q_{\mathrm{x}}$ (intersegment) direction for a full-polarized measurement in the $7.5 \mathrm{mT}$ field condition. (e) Spin-flip scattering cut along the $Q_{\mathrm{y}}$ (interwire) direction for a full-polarized measurement in the $7.5 \mathrm{mT}$ field condition.

to an applied field. In Figure 1c, 1d, and 1e, we can see examples of NSF scattering perpendicular to the nanowires (along Y), SF scattering perpendicular to the wires (along $Y$ ), and SF scattering parallel to the wires (along $X$ ), respectively. By taking such measurements in different applied fields, a 3D field-dependent picture of the magnetic configuration 
and moment orientation may be obtained from the locations of the peaks in each cross section. The SF peak near $Q_{\mathrm{x}}=0.165 \mathrm{~nm}^{-1}$ in Figure 1d, for example, provides definitive evidence of antiparallel alignment of the perpendicular to the nanowire component of the magnetization from one segment to the next (along the nanowire) in low fields.

In Figure 2a, a schematic illustrates the important magnetic interactions within the system as determined from PASANS. Among the segments, the perpendicular magnetic moments interact ferromagnetically (parallel alignment) between wires, but antiferromagnetically (antiparallel alignment) along the wire. On the other hand, when magnetic moments align along the wire, they interact antiferromagnetically between wires. Balancing these interactions results in complex magnetic structures as the applied magnetic field along the wires is increased. At zero field, we have the structure shown in Figure $2 \mathrm{~b}$. As the field increases, wires align into the fan-like and then antiferromagnetic structures shown in Figure $2 \mathrm{c}$ and $2 d$. Finally, at very high fields, all moments eventually line up with the applied field.

In the nanowire geometry used here, competing interactions give rise to complex magnetic structures including antiferromagnetic interwire stripes, in-plane interwire ferromagnetism, and intrawire fan structures, which are highly dependent on the external field. Based on the SANS results, we developed a theoretical framework for accurately treating interwire magnetic interactions in high-density nanowire arrays. These calculations show that all the observed field-dependent behavior, depicted in Figs. 2 b-e, may be accounted for by a combination of dipole and Zeeman interactions, making our findings readily generalizable to other nanowire arrays with a variety of geometries.

The modeling, theory, and PASANS observations suggest that magnetic domain structures in aligned arrays of segmented nanowires can be exploited in the design of nanowirebased devices. However, the ability to probe and understand the magnetic structures formed through interwire and intersegment interactions will be critical to their eventual incorporation into 3D magnetic devices. This work therefore represents an important step in the development of devices based on segmented magnetic nanowire arrays. (a)

(b)
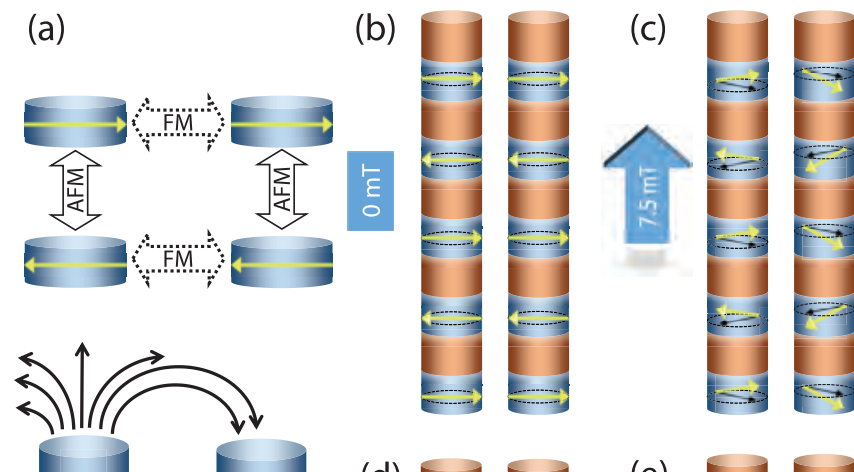

(d)
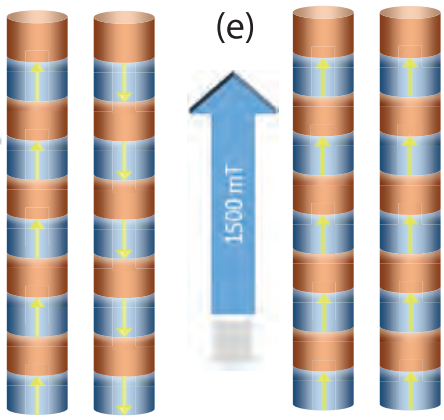

FIGURE 2: (a) Schematic of the magnetic interactions present in the studied nanowires alongside proposed magnetic structures at applied fields of (b) $0 \mathrm{mT}$, (c) $7.5 \mathrm{mT}$, (d) $700 \mathrm{mT}$, and (e) $1500 \mathrm{mT}$.

\section{References}

[1] S. S. P. Parkin, M. Hayashi, L. Thomas, Science 320, 190 (2008).

[2] D. A. Allwood et al., Science 309, 1688 (2005).

[3] Y. P. Ivanov et al., ACS Nano 10, 5326 (2016).

[4] A. J. Grutter et al., ACS Nano 10.1021/acsnano.7b03488 (2017). 


\title{
Enhancing magnetic order in topological insulators with antiferromagnets
}

\author{
Q. L. He, ${ }^{1}$ X. Kou,${ }^{1}$ A. J. Grutter, ${ }^{2}$ G. Yin,${ }^{1}$ L. Pan, ${ }^{1}$ X. Che,${ }^{1}$ Y. Liu, ${ }^{1}$ T. Nie, ${ }^{1}$ B. Zhang, ${ }^{3}$ S. M. Disseler, ${ }^{2}$ \\ B. J. Kirby, ${ }^{2}$ W. Ratcliff II, ${ }^{2}$ Q. Shao, ${ }^{1}$ K. Murata, ${ }^{1}$ X. Zhu, ${ }^{1}$ G. Yu, ${ }^{1}{ }^{1}$. Fan,${ }^{1}$ M. Montazeri, ${ }^{1}$ X. Han, ${ }^{3}$ \\ J. A. Borchers, ${ }^{2}$ and K. L. Wang ${ }^{1}$
}

T opological insulators (TIs) have emerged as an exciting new opportunity in the search for materials systems that can serve as the building blocks for new ultralow-power logic devices and quantum computers. In particular, Tls that have been engineered to possess desirable magnetic properties are prime candidates for use in quantum computing or in next generation "spintronic" logic devices, which use magnetic spins as information carriers rather than relying on charge alone $[1,2]$. A great deal of effort has gone into achieving room temperature magnetism in Tls, either through magnetic doping or proximity coupling to adjacent ferromagnetic layers [3]. Doping may readily induce a high-temperature magnetic phase, but the high concentration of dopants necessary eventually disrupts the topological order of the system. Proximity interactions between ferromagnets have recently been reported to induce a high-temperature magnetization in a Tl, but such systems are extremely hard to characterize accurately due to the way the ferromagnet dominates the signal. Thus, convincing demonstrations of high-temperature magnetic states in these systems above $30 \mathrm{~K}$ have remained elusive.

A potential solution to this dilemma is the incorporation of antiferromagnetic systems that can mimic the spin-polarized interfacial interactions of a ferromagnet. Antiferromagnets, which consist of magnetically ordered spins in an antiparallel configuration rather than the parallel arrangement of a ferromagnet, possess no net magnetization. Further, the magnetic spins of an antiferromagnet typically respond more weakly to an applied field than do those of a ferromagnet. Thus, at an antiferromagnet/Tl interface, the magnetic signal from the TI will be much easier to resolve conclusively. Further, devices based on antiferromagnetic systems are predicted to operate at higher frequencies and be less sensitive to external magnetic fields, suggesting a wide range of exciting new applications.

In order to achieve proximity interactions between antiferromagnets and TIs, thin film superlattices and heterostructures composed of the antiferromagnet chrome antimonide ( $\mathrm{CrSb}$ ) and the TI bismuth antimony telluride $\left[(\mathrm{Bi}, \mathrm{Sb})_{2} \mathrm{Te}_{3}\right]$ were fabricated [4]. CrSb was selected because it provides a unique combination of growth compatibility with TI structures and magnetic structure in which the spins are aligned ferromagnetically within the layer but antiparallel

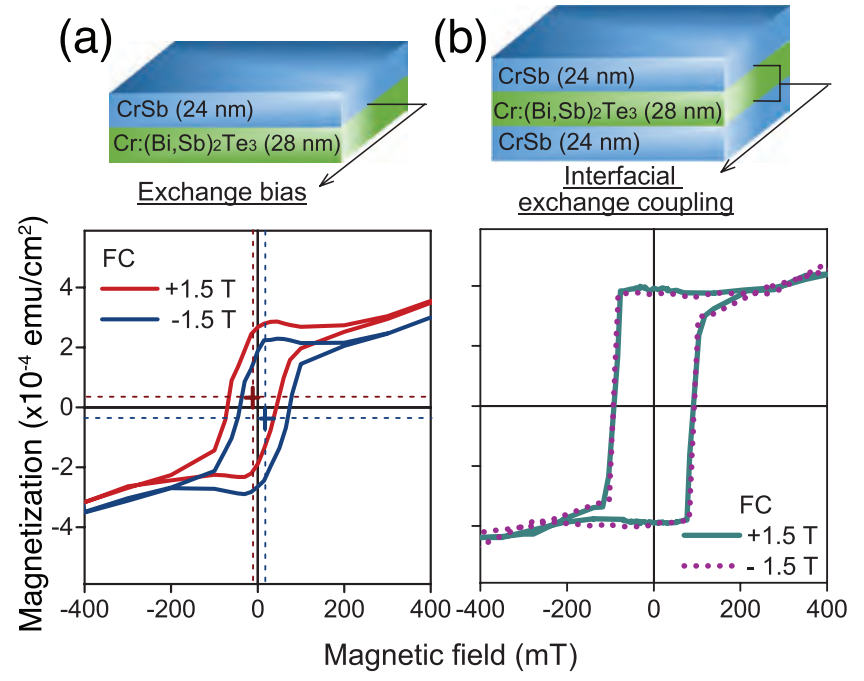

(c)

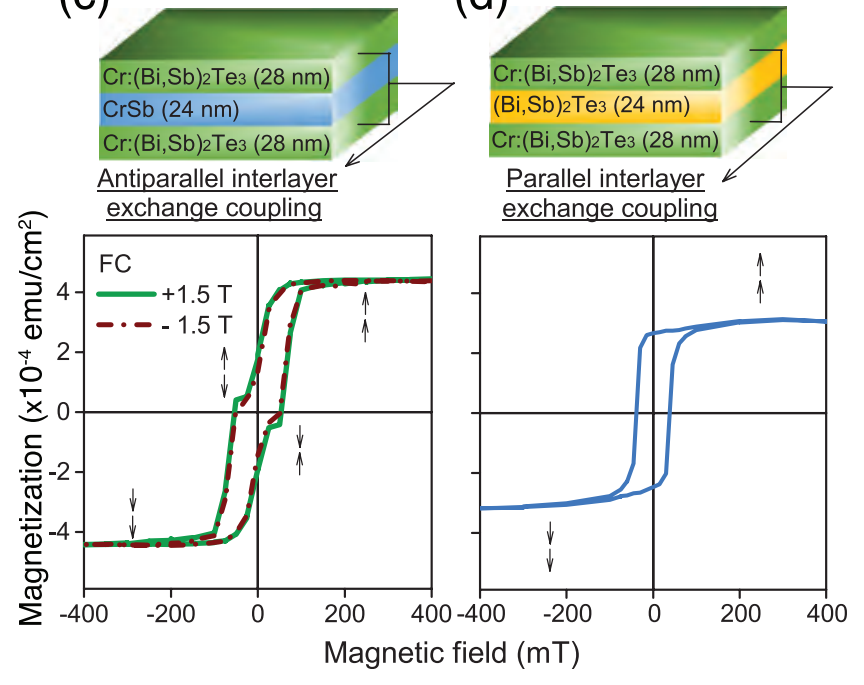

FIGURE 1: (a)-(d) Magnetization vs. applied field measurements for the bilayers and trilayers studied alongside schematics of each sample geometry $\left(1 \mathrm{emu} / \mathrm{cm}^{3}=10^{3} \mathrm{~A} / \mathrm{m}\right)$.

between layers. All Tl layers were lightly doped with $\mathrm{Cr}$ such that the Tl layers were magnetic below $30 \mathrm{~K}$, but the desired topological properties were preserved. Samples studied included bilayers, trilayers, and superlattices. Such 
a combination of different sample geometries allowed the study of individual interfaces in the bilayers, interfaces of sandwiched layers in the trilayers, and possible long-range interactions in the superlattices.

Through characterization of the sample electronic and bulk magnetic properties, it was immediately apparent that the $\mathrm{TI} / \mathrm{CrSb}$ systems were dramatically modified through growth alongside each other (Figure 1). Magnetoresistance, SQUID, and Hall Effect measurements, which examine the magnetic field dependence of electrical resistance in a material, revealed signatures of a net magnetic moment in the samples up to temperatures of $90 \mathrm{~K}$. This represents a threefold increase in the ordering temperature relative to those of the pure $\mathrm{Cr}$-doped TI systems. Furthermore, the enhancement of the magnetic order was found to depend strongly on the sample geometry. Specifically, while magnetic exchange bias is observed in bilayer samples, it disappears in trilayers, which instead exhibit a surprising double-switching behavior upon magnetization reversal that is indicative of interlayer exchange coupling. When sandwiched between two TI layers, the intervening CrSb layer appears to mediate a magnetic interaction between the top and bottom TI layers. In superlattices, this effect is greatly enhanced with increasing thickness, and our findings suggest that the interlayer exchange coupling plays a significant role in enhancing the magnetic ordering temperature of the TI/CrSb system. As shown in Figure 2, increasing the number of repeats in the superlattice results in a rapid ordering temperature increase, which eventually plateaus near $90 \mathrm{~K}$ for 10 bilayer repeats.

Of course, modifications to the magnetic ordering temperature in a two-component system may be attributed to a change in either the $\mathrm{Tl}$ layer, the $\mathrm{CrSb}$ layer, or both. The magnetic transition observed at $90 \mathrm{~K}$ could also represent a dramatic reduction in the $\mathrm{CrSb}$ ordering temperature due to interfacial defects or finite size effects. It is therefore critical to understand the magnetic behavior of the individual layers. To characterize the antiferromagnetic ordering in the CrSb layers we used temperature-dependent neutron diffraction to probe the intensity of the magnetic diffraction peaks. These data confirmed that the CrSb moments remain aligned primarily along the $c$-axis as in the bulk system.

To better understand the individual layer behavior and interlayer exchange coupling, polarized neutron reflectometry (PNR) measurements were performed using the PBR instrument at the NIST Center for Neutron Research on a bilayer and a $\times 10$ repeat superlattice. PNR reveals the structural and magnetic depth profile of these layered films. As shown in Figure 2, modeling of the PNR data demonstrates that a net magnetization appears in the $\mathrm{CrSb}$ layer when these layers are sandwiched between two TI layers. On the other hand, when the CrSb has only a single top or bottom interface with a TI layer, no net magnetization in observed. These findings support the interpretation of an

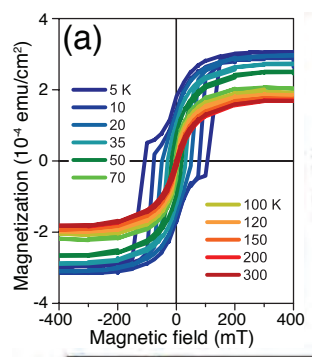

(b)
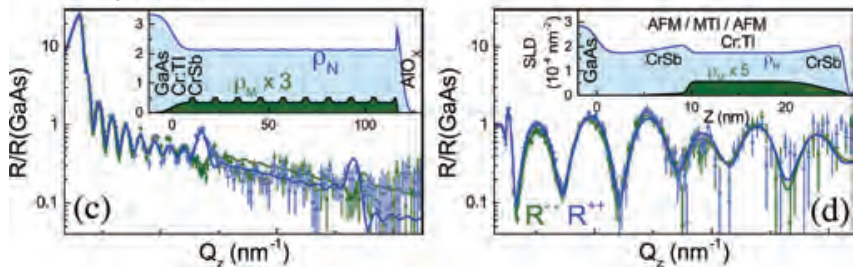

FIGURE 2: (a) Temperature dependent magnetization vs. applied field for a 10-repeat superlattice sample. (b) Temperature dependence of the magnetic ordering temperature $\left(\mathrm{T}_{\mathrm{c}}\right)$ vs. number of superlattice repeats. (c) Polarized neutron reflectometry data and corresponding depth profiles for a 10-repeat superlattice and (d) a trilayer sample. Green and blue curves represent the reflectivity for neutron spin down and up, respectively.

interlayer exchange coupling in which the TI layers couple to the $\mathrm{CrSb}$, changing its magnetic structure. These changes propagate through the $\mathrm{CrSb}$ and are reinforced by interactions with the TI layer on the other side. In this way, long-range coupling interactions are stabilized that increase the magnetic ordering temperature of the entire system. Thus the enhanced magnetic ordering temperature is the result of enhanced coupling of magnetic spins in both the $\mathrm{TI}$ and $\mathrm{CrSb}$.

This work demonstrates the surprising power of antiferromagnetic layers to tune magnetic interactions in topologically nontrivial systems. It must be noted, however, that such results require the ability to grow flat structures with incredibly sharp interfaces. The observed interactions are enabled because of the spin-polarized planes present in the $\mathrm{CrSb}$. However, even a slight nonuniformity of thickness or a significant intermixing at the interface will result in the TI layer interaction with the $\mathrm{CrSb}$ spins aligned in opposite directions. Therefore, the system must truly be an atomically flat thin film. As the ability to grow more films of exceptional quality improves, we anticipate the emergent field of topological antiferromagnetic spintronics will expand greatly, which will have important implications for magnetic logic devices, quantum computing, and beyond.

\section{References}

[1] Y. Fan et al., Nature Materials 13 (7), 699 (2014).

[2] C.-Z. Chang et al., Science 340 (6129), 167 (2013).

[3] F. Katmis et al., Nature 533, 513 (2016).

[4] Q. L. He et al., Nature Materials 16 (1), 94 (2017). 


\section{Atomic scale engineering of multiferroicity in LuFeO $3 / \mathrm{LuFe}_{2} \mathrm{O}_{4}$ superlattices}

J. A. Mundy, ${ }^{1}$ C. M. Brooks, ${ }^{2}$ M. E. Holtz, ${ }^{1}$ J. A. Moyer, ${ }^{3}$ H. Das, ${ }^{1}$ A. F. Rébola, ${ }^{1}$ J. T. Heron, ${ }^{2,4}$ J. D. Clarkson, ${ }^{5}$ S. M. Disseler, ${ }^{6}$ Z. Liu, ${ }^{5}$ A. Farhan, ${ }^{7}$ R. Held, ${ }^{2}$ R. Hovden, ${ }^{1}$ E. Padgett, ${ }^{1}$ Q. Mao, ${ }^{1}$ H. Paik, ${ }^{2}$ R. Misra, ${ }^{8}$ L. F. Kourkoutis, ${ }^{1,9}$ E. Arenholz, ${ }^{7}$ A. Scholl, ${ }^{7}$ J. A. Borchers, ${ }^{6}$ W. D. Ratcliff, ${ }^{6}$ R. Ramesh, ${ }^{5,10,11}$ C. J. Fennie, ${ }^{1}$ P. Schiffer, ${ }^{3}$ D. A. Muller, ${ }^{1,9}$ and D. G. Schlom ${ }^{2,9}$ and magnetic order. For technological applications, we desire that these two order parameters be tightly coupled. Unfortunately, despite years of searching, there has been a paucity of materials for which both orders exist at room temperature, while evincing control of magnetism by an applied voltage [1, 2]. Thus, engineering artificial multiferroics using atomic-scale heterostructures is rapidly becoming an attractive alternative. In this work, we have combined two crystallographically similar but poor multiferroic materials to engineer a new family of ferromagnetic multiferroic heterostructures [3].

These heterostructures are based on the family of hexagonal compounds $\mathrm{RMO}_{3}$ which have been known multiferroics since their discovery in the early 1960s. In these materials, the ferroelectric moment results from a structural transition, typically above $700 \mathrm{~K}$, in which the rare-earth $R$ and $\mathrm{O}$ ions displace along the $c$-axis by disproportionate amounts leading to a net charge displacement. The magnetic transition metal $M$ sites form a triangular lattice in the plane, with superexchange interactions leading to antiferromagnetic ordering only below $150 \mathrm{~K}$ [4]. Thus, despite exhibiting both magnetism and ferroelectricity at low temperatures, the two orders are only weakly linked, and this material is not expected to be a useful multiferroic near room temperature [5].

On the other hand, $\mathrm{LuFe}_{2} \mathrm{O}_{4}$ is a hexagonal material with lattice constants similar to those of the high temperature phase of the $R M \mathrm{O}_{3}$ compounds, but it ferrimagnetically orders below $240 \mathrm{~K}$ and does not undergo any inversion-symmetry breaking structural transitions. In fact, the $\mathrm{LuFe}_{2} \mathrm{O}_{4}$ and $\mathrm{LuFeO}_{3}$ crystal structures may be envisioned as simply alternating planes of $\mathrm{Fe}-\mathrm{O}(\mathrm{A})$ and $\mathrm{Lu}-\mathrm{O}$ (B) layers, with $\mathrm{LuFeO}_{3}$ defined by the pattern $\mathrm{ABAB} \ldots$, and

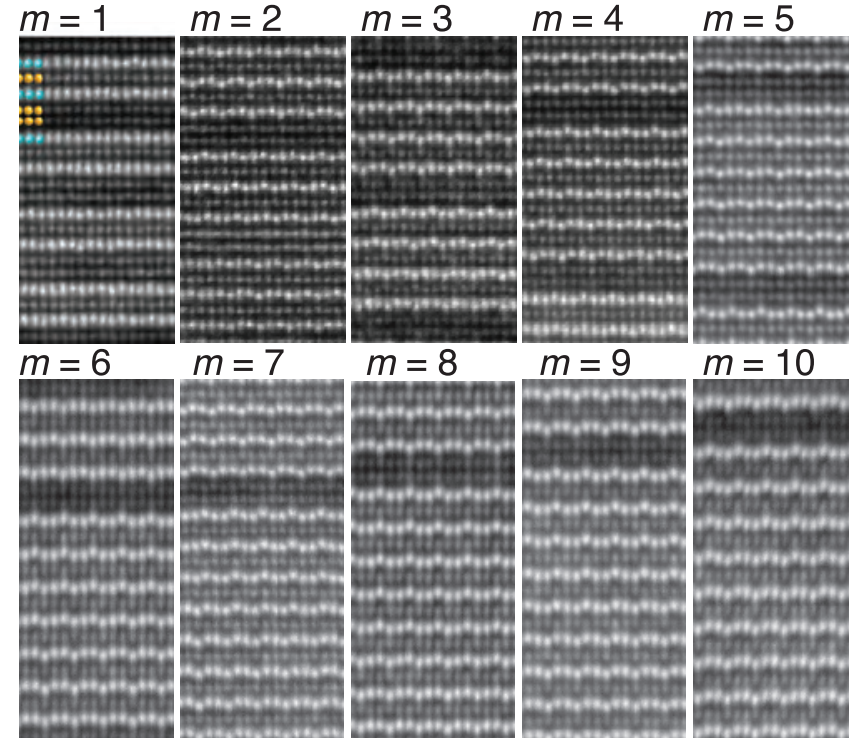

FIGURE 1: HAADF-STEM images of MBE grown $\mathrm{LuFeO}_{3}-\mathrm{LuFe}_{2} \mathrm{O}_{4}$ superlattices [3]. The $m$ indicates the number of sequential $\mathrm{AB}\left(\mathrm{LuFeO}_{3}\right)$ units between each double $\mathrm{Fe}-0$ layer [1]. Lutetium (Lu) is shown as turquoise and iron (Fe) is shown as yellow.

$\mathrm{LuFe}_{2} \mathrm{O}_{4}$ by $\mathrm{AABAABAAB} \ldots$... Thus, using epitaxial or syntactic growth of individual Fe-O and Lu-O layers we can effectively interpolate between the two compounds. To do so, we have used oxide molecular beam epitaxy (MBE) which allows precise control of atomically sharp interfaces over large scales, with imaging from high-angle annular dark field scanning transmission electron microscopy (HAADF-STEM) shown in Figure 1.

Interestingly, we find that introducing more $\mathrm{LuFeO}_{3}$ increases the ferromagnetic ordering temperature of the $\mathrm{LuFe}_{2} \mathrm{O}_{4}$-like layers, from $220 \mathrm{~K}$ to nearly room temperature $[281 \mathrm{~K}$ in the 

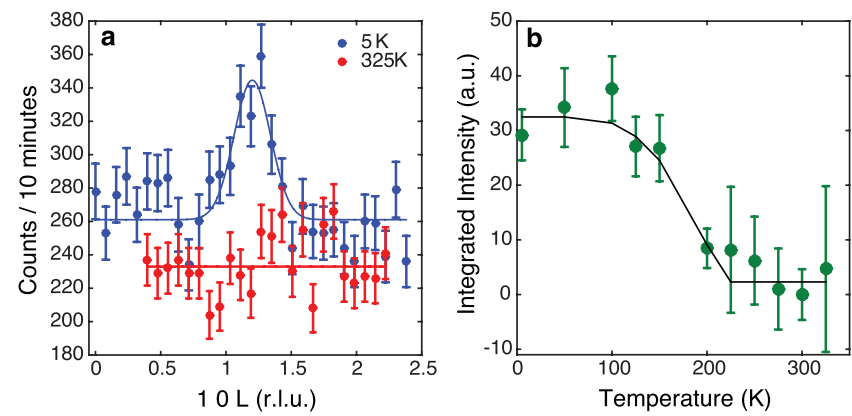

FIGURE 2: Neutron diffraction of the $\left(\mathrm{LuFeO}_{3}\right)_{6} /\left(\mathrm{LuFe}_{2} \mathrm{O}_{4}\right)_{2}$ superlattice [3]. (a) Magnetic reflections for the $\left(\mathrm{LuFeO}_{3}\right)_{6} /\left(\mathrm{LuFe}_{2} \mathrm{O}_{4}\right)_{2}$ superlattice were observed using neutron diffraction by scanning along the [10L] direction in reciprocal space, expressed in reciprocal lattice units (r. l. u.) at several temperatures between $5 \mathrm{~K}$ and $325 \mathrm{~K}$. A single peak is observed that changes considerably in intensity between $5 \mathrm{~K}$ and room temperature. The offset from the (101) position is due to a slight misalignment of the sample. (b) Integrated intensity of the (101) magnetic reflection for the $\left(\mathrm{LuFeO}_{3}\right)_{6} /\left(\mathrm{LuFe}_{2} \mathrm{O}_{4}\right)_{2}$ superlattice as a function of temperature. The solid line is the mean-field fit. Error bars in $a$ and $b$ represent one standard deviation.

$\left(\mathrm{LuFeO}_{3}\right)_{9} /\left(\mathrm{LuFe}_{2} \mathrm{O}_{4}\right)_{1}$ sample], uniformly as a function of $m$ (the number of $\mathrm{LuFeO}_{3}$ layers). Furthermore, we find that by maximizing $T_{\mathrm{c}}$, both the robustness of the ferroelectric state and the magnetoelectric coupling of the ferromagnetic and ferroelectric moments are also dramatically enhanced. In thin films, there is often the worry that magnetic effects measured by bulk techniques such as SQUID magnetometry may stem from ferromagnetic impurities. Neutron scattering experiments played a critical role in determining that the high transition temperatures observed in these superlattices were intrinsic to the material. In Figure 2, we show an order parameter of the magnetic (101) reflection measured on the BT-4 triple-axis spectrometer.
Finally, we investigated the coupling between the two order parameters. In Figure 3 (a), we show the effects of writing a pattern with a piezoresponse force microscopy (PFM) tip. In Figure 3 (b), we show X-ray magnetic circular dichroic photoemission electron microscopy (XMCD PEEM) images (measured at $200 \mathrm{~K}$, below the magnetic transition temperature), which reveal that the magnetic order in these materials follows the ferroelectric ordering. In Figure 3 (c), we show that at high temperatures, this order is markedly reduced, yet still remains (in Figure 3 (d), we show line cuts through the signal at the two different temperatures).

This work demonstrates a strategy that is capable of developing multiferroic materials with strong coupling and high transition temperatures. The design principle was to imprint the ferroelectric "rumpling" onto $\mathrm{LuFe}_{2} \mathrm{O}_{4}$ and establish ferroelectricity in that compound. Density Functional Theory (DFT) calculations suggest that this rumpling also serves to increase the interaction between moments in the $\mathrm{LuFe}_{2} \mathrm{O}_{4}$ layer and thus increase the transition temperature. The magnetic reflection we observed was also consistent with the lowest energy magnetic structure predicted by DFT.

\section{References}

[1] N. A. Spaldin, M. Fiebig, Science 309, 391 (2005).

[2] W. Eerenstein, N. D. Mathur, J. F. Scott, Nature 442, 759 (2006).

[3] J. Mundy et al., Nature 537, 523 (2016).

[4] S. Disseler et al., Phys. Rev. Lett. 114, 217602 (2015).

[5] S. Disseler et al., Phys. Rev. B 92, 054435 (2015). a

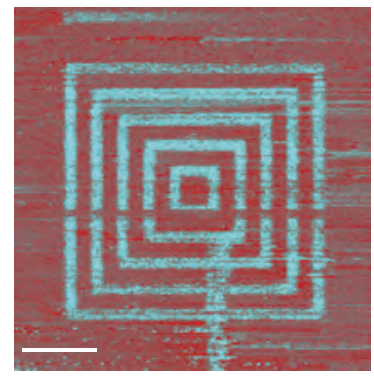

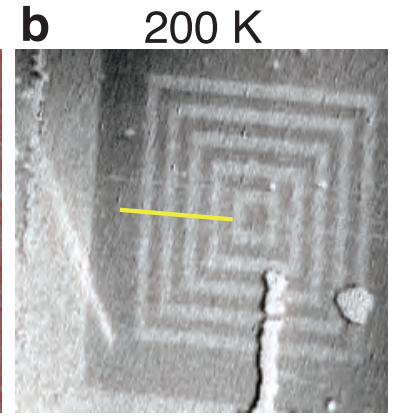
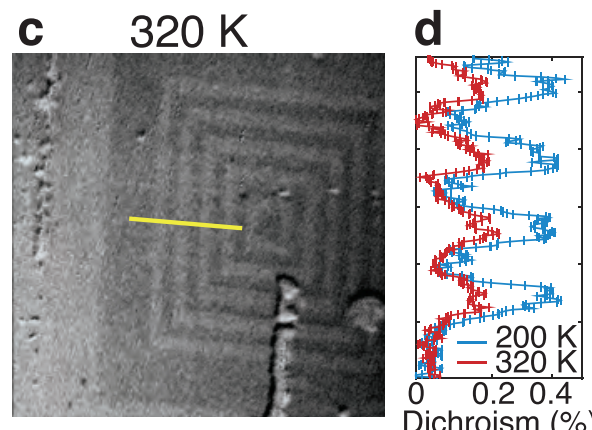

FIGURE 3: Magnetoelectric coupling in the $\left(\mathrm{LuFeO}_{3}\right)_{9} /\left(\mathrm{LuFe}_{2} \mathrm{O}_{4}\right)_{1}$ superlattice [3]. (a) Out-of-plane PFM image at $300 \mathrm{~K}$ of the domain structure following electrical poling using a d.c. bias applied to the proximal tip. The 'up' and 'down' c-oriented domains appear in turquoise and red respectively. Scale bar equals $3 \mu \mathrm{m}$. XMCD PEEM ratio images from the Fe L3 edge acquired at $200 \mathrm{~K}$ (b) and $320 \mathrm{~K}$ (c). The correlation between the electrical poling and magnetic imaging demonstrates electric-field control of ferrimagnetism at $200 \mathrm{~K}$. (d) Comparison of the dichroic signals along the yellow lines in b and c. 


\title{
Nascent magnetic order and quantum fluctuations in a spin orbital liquid
}

\author{
K. W. Plumb, ${ }^{1}$ J. R. Morey, ${ }^{1,2}$ J. A. Rodirguez-Rivera, ${ }^{3,4}$ H. Wu, ${ }^{3}$ A. A. Podlesnyak, ${ }^{5}$
}

T. M. McQueen, ${ }^{1,2,6}$ and C. L. Broholm ${ }^{1,3,5}$

$\mathrm{n}$ magnetic materials both the electronic orbitals and spins can participate in collective behaviors. Typically, degeneracies in the orbital filling are lifted by the Jahn-Teller effect at high temperatures, resulting in a quenching of orbital angular momentum and a frozen pattern of orbital occupation, or orbital ordering. This is followed by collective spin ordering at a lower temperature. However, such a separation of energy scales is not guaranteed and the spin and orbital sectors can influence each other through spin-orbit coupling and exchange interactions. When spin and orbital energy scales are of comparable strength, then it is even possible to avoid completely any spin or orbital ordering and form a spin-orbital quantum liquid state. In this state both the spin and orbital degrees of freedom fluctuate collectively at zero temperature.

$\mathrm{FeSC}_{2} \mathrm{~S}_{4}$ is a cubic spinel where $\mathrm{Fe}^{2+}$ ions carry both a spin and orbital degree of freedom. The spin and orbital energy scales are of nearly equal strength, and previous work has uncovered evidence that the ground state may be a spin-orbital liquid [1, 2] Using a combination of neutron powder diffraction and neutron inelastic scattering techniques, we have recently found evidence for a coexistence of weak spin and orbital order with strong quantum fluctuations in $\mathrm{FeSc}_{2} \mathrm{~S}_{4}$ [3]. The analysis of our neutron diffraction and spectroscopic data has provided detailed atomic scale insight into the nature of spin-orbital ordering. Our observations indicate that the ground state of $\mathrm{FeSc}_{2} \mathrm{~S}_{4}$ lies near a quantum critical point separating a spin and orbitally ordered state from a spin-orbital liquid.

In Figure 1 (a) we present a false color map of the temperature dependent neutron diffraction for $\mathrm{FeSC}_{2} \mathrm{~S}_{4}$ measured using the MACS spectrometer. The high sensitivity measurements reveal magnetic Bragg reflections that appear below a temperature $T_{\mathrm{c}}=11.8 \mathrm{~K}$. The temperature dependent ordered moment extracted from the integrated Bragg intensity smoothly increases with decreasing temperature, with a critical exponent of $\beta=0.54$ and saturated moment of $1.9 \mu_{\mathrm{B}} / \mathrm{Fe}^{2+}$, as is shown in Figure 1 (c). In Figure 1 (b) we show a detailed view of the (100) magnetic reflection at $1.8 \mathrm{~K}$. The peak is broader than the instrumental resolution, and we find a resolution-corrected peak half-width at half-maximum of $0.019(6) \AA^{-1}$, corresponding to a magnetic
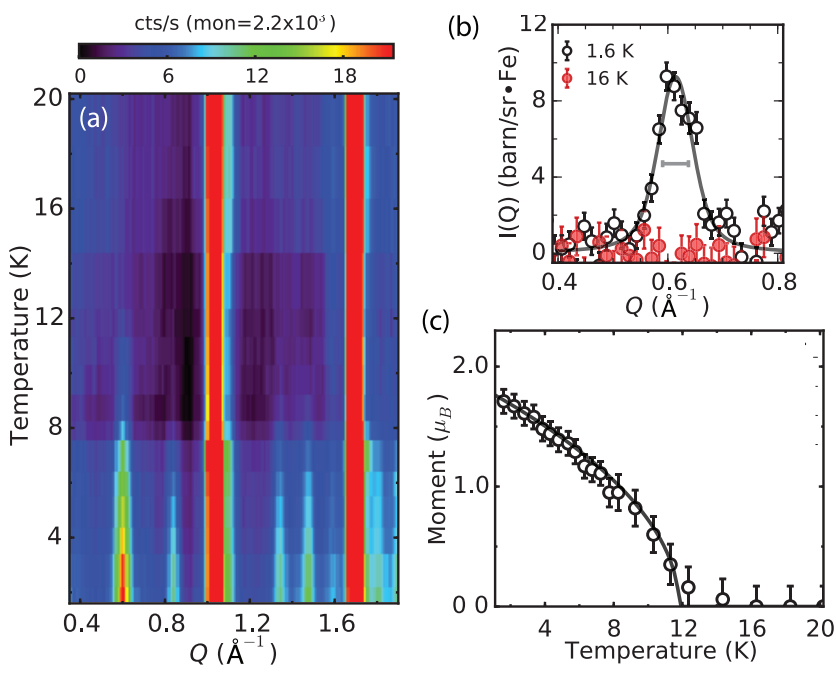

FIGURE 1: (a) Temperature dependent neutron diffraction measured on MACS. (b) Detailed view of the (100) magnetic Bragg reflection; horizontal bar indicates the instrumental resolution. (c) Temperature dependent ordered moment extracted from the MACS diffraction data; solid line is a fit to a magnetic order parameter with a critical temperature of $11.8(2) \mathrm{K}$.

correlation length of $53(16) \AA$. This finite correlation length is believed to result from magnetic exchange disorder that is built into the material by a higher temperature orbital ordering transition.

Neutron diffraction is not necessarily directly sensitive to orbital ordering, or orbital degeneracy breaking, but a particular orbital ordering pattern can be indirectly inferred through careful measurement of the crystal structure. To explore the possibility of orbital ordering in $\mathrm{FeSC}_{2} \mathrm{~S}_{4}$, we have performed neutron powder diffraction measurements using the BT-1 diffractometer. The results of this measurement are shown in Figure 2 (a). The crystallographic unit cell maintains its cubic metric at low temperature, meaning that the maximum change in the cubic lattice parameter $a$ that is consistent with our measurement is $\Delta a / a=0.0002$ (2). However, Rietveld refinement of our diffraction data yields a systematic discrepancy between measured and calculated peak intensities for the cubic crystal structure. 
(a)

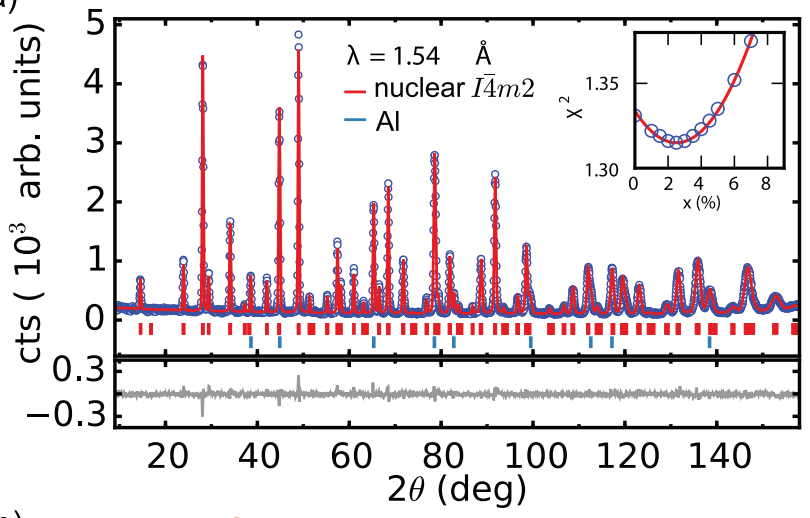

(b)

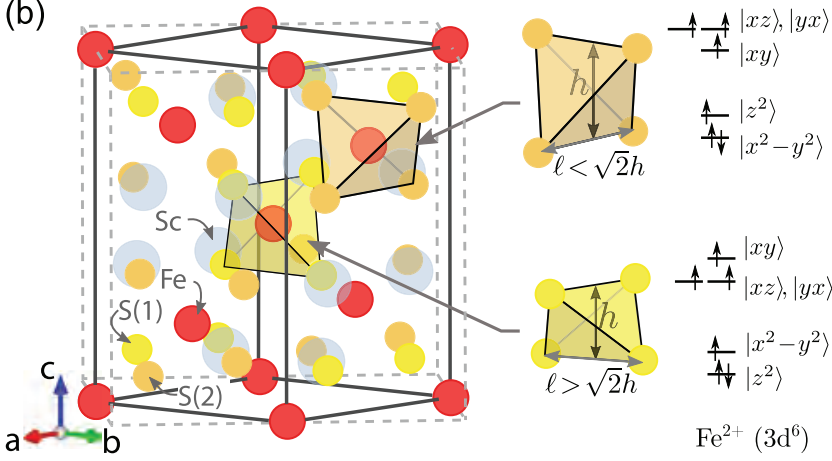

FIGURE 2: (a) Powder neutron diffraction measured on BT-1. Solid red line is a Rietveld refinement of the tetragonal cell; inset shows a goodness of fit parameter versus Fe-Sc anti-site inversion. (b) Proposed tetragonal crystal structure; the parent cubic cell is shown as a light grey dashed line. There are two distinct sulfur sites resulting in two different $\mathrm{Fe}^{2+}$ coordination environments and orbital occupations shown on the right.

By testing the maximal subgroups of the cubic structure, we find that the measured diffraction data are much better described using a tetragonal structure containing two distinct Fe sites, each forming a centered tetragonal sub-lattice as shown in Figure 2 (b). Within each sub-lattice the Fe coordinating $\mathrm{S}$ tetrahedra are alternately compressed and elongated along the (100) direction. This distortion lifts the orbital degeneracy of the cubic cell, but in a different way on each of the two Fe sites.

The tetragonal distortion is an essential piece of information required for a consistent understanding of both the magnetic order and the pattern of inelastic magnetic scattering. In Figure 3 we show a summary of the powder averaged neutron inelastic spectra measured on the MACS spectrometer as well as the magnetic diffraction pattern. Rietveld refinement of the magnetic structures using the tetragonal crystallographic unit cell reveals a collinear antiferromagnet with a strongly reduced ordered moment of $1.9 \mu_{B}$, which indicates strong quantum fluctuations in this insulating magnet. A close up of the lowest $Q$ magnetic Bragg reflections is shown in panel (c) of Figure 3, and the corresponding low energy magnetic excitation spectra are in panel (b). If one uses the cubic crystal structure, then the two lowest- $Q$ Bragg reflections occur at symmetry equivalent wave vectors. However, the presence of strong inelastic intensity around the first magnetic reflection combined with the absence of such intensity around the second implies

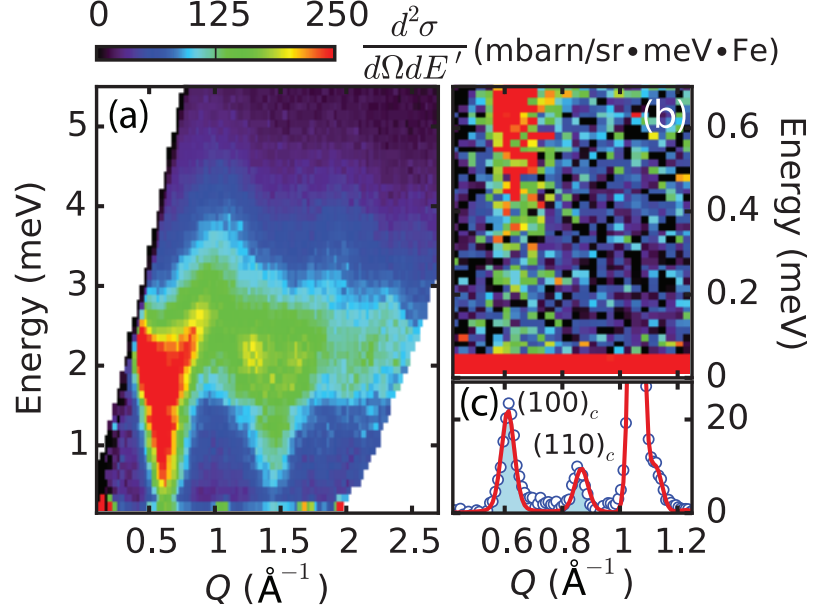

these two positions are not symmetry equivalent. Therefore, the magnetic ordering must exist in a tetragonal structure, which breaks the symmetry of the cubic cell. Indeed, the powder-averaged magnetic excitation spectrum is well described by a spin-wave model in the tetragonal cell [3]. Thus, it is the magnetic excitation spectra that pinpoint the orbital ordering pattern in $\mathrm{FeSC}_{2} \mathrm{~S}_{4}$ and demonstrate the intimate connection between spin and orbital degrees of freedom.

The powder-averaged magnetic excitation spectrum in Figure 3 (a) reveals another important feature. The energy and momentum integrated neutron inelastic signal takes up $80 \%$ of the available magnetic spectral weight. This finding is at odds with a conventional Neél ordered state and indicates that the ground state of $\mathrm{FeSC}_{2} \mathrm{~S}_{4}$ is dominated by quantum fluctuations.

In short, our finding of magnetic order in $\mathrm{FeSc}_{2} \mathrm{~S}_{4}$ sheds new light on this material. We show the presumed spin-orbital liquid actually supports a small staggered magnetization; however, the magnetic order is not conventional, and we can clearly place $\mathrm{FeSC}_{2} \mathrm{~S}_{4}$ in proximity to a quantum critical point where the staggered magnetization melts. Our study thus opens a new chapter for experimental and theoretical investigations into the class of quantum phase transitions involving entangled spin and orbital degrees of freedom.

\section{References}

[1] A. Krimmel, M. Mucksch, V. Tsurkan, M. M. Koza, H. Mutka, A. Loidl, Phys. Rev. Lett. 94, 237402 (2005).

[2] G. Chen, L. Balents, A. P. Schnyder, Phys. Rev. Lett. 102, 096406 (2009).

[3] K. W. Plumb, J. Morey, J. A. Rodriguez-Rivera, H. Wu, A. A. Podlesnyak, T. M. McQueen, C. L. Broholm, Phys. Rev. X 6, 041055 (2016). 


\section{Orbital-exchange and fractional quantum excitations in an f-electron metal $\mathbf{Y b}_{2} \mathrm{Pt}_{2} \mathbf{P b}$}

L. S. Wu, ${ }^{1,2,3}$ W. J. Gannon, ${ }^{1,2,4}$ I. A. Zaliznyak, ${ }^{2}$ A. M. Tsvelik, ${ }^{2}$ M. Brockmann, ${ }^{5,6}$ J. -S. Caux, ${ }^{6}$ M. S. Kim, ${ }^{2}$ Y. Qiu, ${ }^{7}$ J. R. D. Copley, ${ }^{7}$ G. Ehlers, ${ }^{3}$ A. Podlesnyak, ${ }^{3}$ and M. C. Aronson ${ }^{1,2,4}$

$\mathrm{F}$ ractional magnetic excitations, such as one-dimensional spinons, are incontrovertible evidence for the quantum nature of a system, and it is widely believed that small magnetic moments are required, such as those originating from a transition metal ion with $d$-electrons. Our neutron scattering experiments on the DCS instrument unambiguously reveal exactly these exotic quantum states in a much different sort of system, $\mathrm{Yb}_{2} \mathrm{Pt}_{2} \mathrm{~Pb}$ [1]. Our observation of spinons in this material is novel because the magnetic moments hosting these excitations are very large, arising from orbitally dominated $f$-electron states of $\mathrm{Yb}^{3+}$. In $3 d$-electron materials with spin-1/2 moments the orbital angular momentum is quenched and spinons are typically associated with the separation of the charge and spin degrees of freedom. In $\mathrm{Yb}_{2} \mathrm{Pt}_{2} \mathrm{~Pb}$, magnetic moments have predominantly orbital origin, and spinons bring about the separation of the charge and orbital degrees of freedom.

Shown in Fig. $1 \mathrm{~A}$ is the crystal structure of $\mathrm{Yb}_{2} \mathrm{Pt}_{2} \mathrm{~Pb}$ [2], where the unusual properties emerge from the $\mathrm{Yb}$-ladders that extend along the $c$-axis. The strong spin-orbit coupling combines spin and orbital degrees of freedom into a large, anisotropic $J=7 / 2 \mathrm{Yb}$ moment, which is dominated by $L=3$. The crystal electric field $(C E F)$ lifts the eightfold $(2 J+1=8)$ degeneracy of the $J$-multiplet and results in doublet ground states of nearly pure $\mid \pm 7 / 2>$. Inelastic neutron scattering experiments on $\mathrm{Yb}_{2} \mathrm{Pt}_{2} \mathrm{~Pb}$ reveal a spinon continuum dispersing along $[00 \mathrm{~L}]$ (Fig. 2A) [1], typical of the $S=1 / 2$ Heisenberg-lsing XXZ spin Hamiltonian:

$H=J_{0} \sum_{\mathrm{n}} S_{\mathrm{n}}^{\mathrm{x}} S_{\mathrm{n}+1}^{\mathrm{x}}+S_{\mathrm{n}}^{\mathrm{y}} S_{\mathrm{n}+1}^{\mathrm{y}}+\Delta S_{\mathrm{n}}^{\mathrm{z}} S_{\mathrm{n}+1}^{\mathrm{z}}$,

where $J_{0}$ is the spin-exchange coupling, and $\Delta$ is its anisotropy. A modest gap was observed in the spinon spectrum, $M(Q, E)$, that is much smaller than the excitation bandwidth, suggesting weak Ising anisotropy. Reflecting the weak interactions among orthogonally aligned ladders of the Shastry-Sutherland Lattice (SSL) geometry in the ab-plane [3], a flat dispersion was observed along [HHO] (Fig. 2 (B, C)).
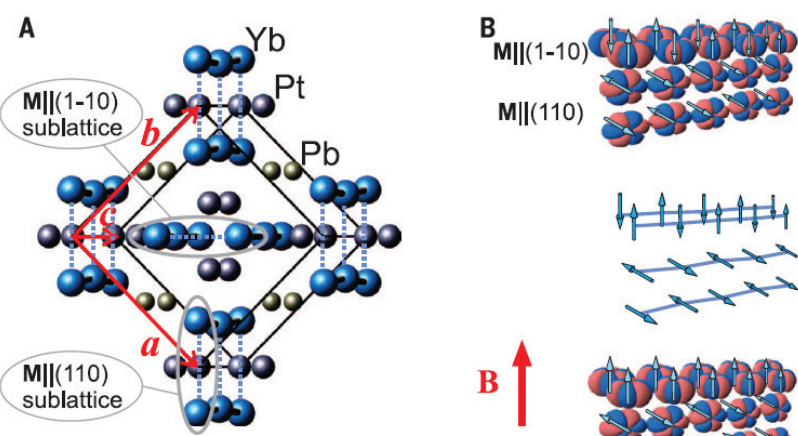

FIGURE 1: (A) Crystal structure of $\mathrm{Yb}_{2} \mathrm{Pt}_{2} \mathrm{~Pb}$. (B): Magnetically ordered structure of $\mathrm{Yb}_{2} \mathrm{Pt}_{2} \mathrm{~Pb}$ without magnetic field (top) and in a $4 \mathrm{~T}$ field (bottom) along the $(-110)$ direction. The $4 f(L=3)$ orbitals are shown at $\approx 1 \mathrm{ppm}$ electronic density for an effective Slater nuclear charge of ${ }^{70} \mathrm{Yb}$. (C) Orbital overlaps for the antiferromagnetic $(H=0)$ and fully saturated $(H=4 \mathrm{~T})$ state.

The overall wave vector dependence of the energy-integrated intensity, $M(Q)$, reveals that the excitations are dominated by longitudinally polarized fluctuations. As demonstrated in Fig. 2D, the spectrum dependence on wave vector, $H$, is well described by the polarization factor with $\mathrm{Yb}$ moments fluctuating along their local Ising axes [1]. This longitudinal character of the spinon excitations in $\mathrm{Yb}_{2} \mathrm{Pt}_{2} \mathrm{~Pb}$ is a direct consequence of the $\mathrm{Yb}$ single ion physics, where strong spinorbital coupling produced an Ising-like orbital anisotropy. Quantitative calibration of $M(Q, E)$ [1] verifies that the neutron inelastic and elastic scattering data satisfies the sum rules, as shown in Fig. 3(A). We find a total moment $M_{\text {total }}$ that is between 3.8 and $4.4 \mu_{\mathrm{B}} / \mathrm{Yb}$ for a wide temperature range from $0.1 \mathrm{~K}$ to $100 \mathrm{~K}$. This suggests that the spinons are entirely responsible for all of the magnetic dynamics in $\mathrm{Yb}_{2} \mathrm{Pt}_{2} \mathrm{~Pb}$. Significantly, the spinon excitation spectrum strongly resembles those found in Heisenberg $S=1 / 2$ spin chain systems [4]. Although individual $\mathrm{Yb}$ magnetic moments are Ising-like, comparison of the model calculation of the 1D 

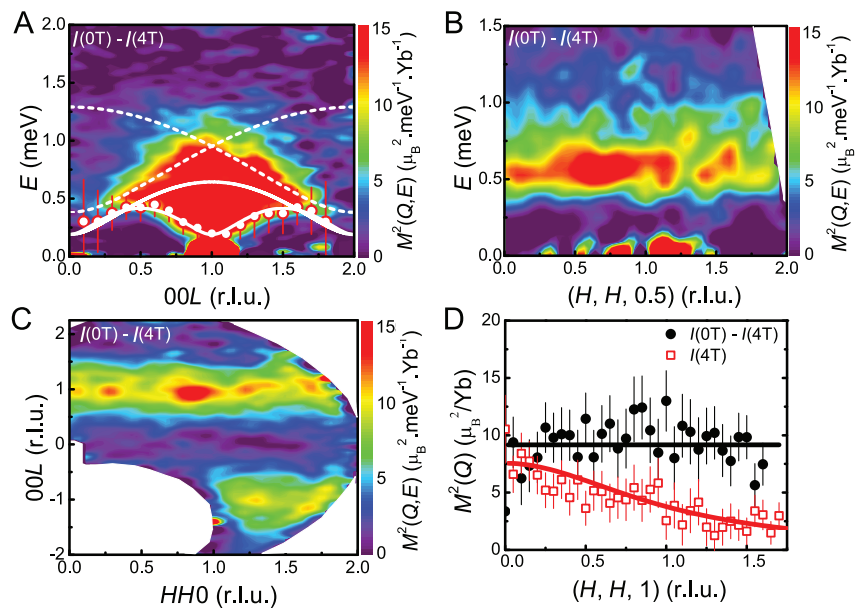

FIGURE 2: (A) The dispersion of the spectrum of magnetic excitations along the $[00 \mathrm{~L}]$ direction in reciprocal space of $\mathrm{Yb}_{2} \mathrm{Pt}_{2} \mathrm{~Pb}$ at $T=0.1 \mathrm{~K}$. The circles mark the onset of the excitation continuum, while the white lines indicate the lower and upper boundaries of the spinon continua. (B) The dispersion of the scattered neutron intensity along the $[H, H, 0.5]$ direction. (C) The partial static structure factor $M(Q)$, obtained by integrating the scattered intensity from $0.15 \mathrm{meV}$ to $1.5 \mathrm{meV}$. (D) Fluctuations along the magnetic field direction, [-110], are perpendicular to the scattering plane and therefore yield intensity that is independent of the wave vector orientation in this plane. The polarization factor reflects the projections of the (110) sublattice moments on the scattering wave vector (red line), indicating that only magnetic fluctuations along the (110) moments contribute to magnetic scattering.

XXZ Hamiltonian to the experimental magnetic susceptibility (Fig.3(B)) and to the spinon scattering spectrum (Fig.3 (C, D)) reveals only a moderate exchange anisotropy is present for the $S=1 / 2$ pseudo-spins, with $\Delta=2.6$ and $J_{0}=0.205 \mathrm{meV}$. The exchange mechanism for the spinons in $\mathrm{Yb}_{2} \mathrm{Pt}_{2} \mathrm{~Pb}$ is necessarily unconventional. We propose that is via the direct exchange of $f$-electrons between neighboring orbitals along the direction of the chains, illustrated in Fig. 1(B, C) [1].

As a rare-earth based intermetallic metal, $\mathrm{Yb}_{2} \mathrm{Pt}_{2} \mathrm{~Pb}$ is an unexpected place to find fractional quantum excitations, since the strong coupling of the spin and orbital degrees of freedom in such materials typically hinders appreciable quantum effects. However, the specific qualities of the $\mathrm{Yb}$ ions in $\mathrm{Yb}_{2} \mathrm{Pt}_{2} \mathrm{~Pb}$ instead enhance quantum effects in spectacular fashion. Previous studies have shown that quantum
A
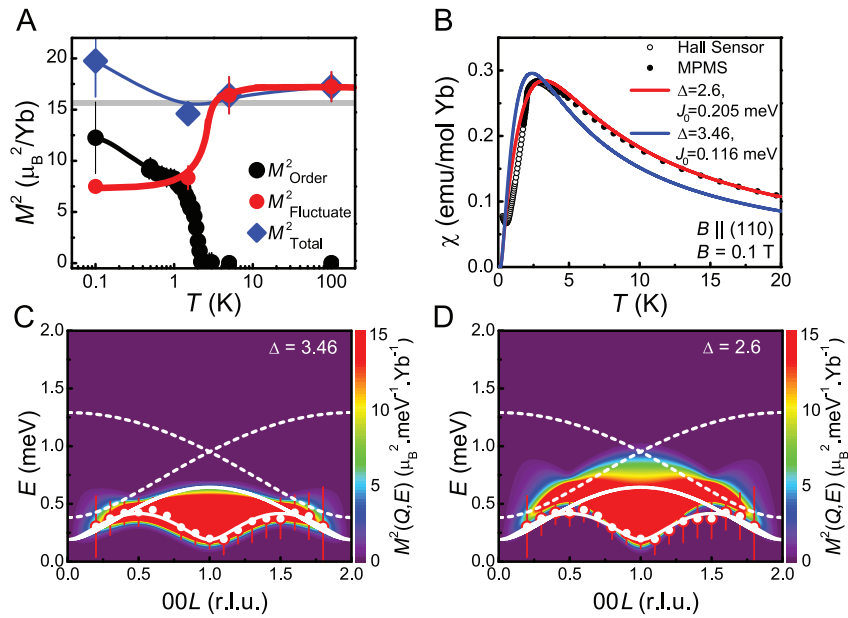

FIGURE 3: (A) Temperature dependencies of the ordered $\mathrm{Yb}$ moment from neutron diffraction measurements (black circles), the fluctuating moment from the energy-and wave-vector-integrated normalized $M(Q, E)$ (red points), and the total moment (blue points). (B) The temperature dependence of the static uniform magnetic susceptibility, which shows good agreement with calculated for the XXZ chain, for $\Delta=2.6$ and $J_{0}=0.205$ (red line). (C), (D) The longitudinal structure factor, $M(Q, E)$, of the XXZ spin-1/2 chain calculated for $\Delta=3.46$ and $J_{0}=0.116 \mathrm{meV}$ (C), and for $\Delta=2.6$ and $J_{0}=0.205 \mathrm{meV}$ (D). The experimentally determined lower boundary is shown (circles) along with the calculated lower and upper 2 spinon (solid lines) and the upper 4 spinon (broken lines) boundaries.

effects can decouple the spin and charge degrees of freedom of electrons, as well as the spin and orbital degrees of freedom in dynamics of magnetic moments [4, 5]. Our measurements offer the first evidence that charge and orbital quantum numbers can also be separated, provide a system with unique access to longitudinal excitations, and open up new avenues for studies, both theoretical and experimental.

\section{References}

[1] L. S. Wu et al., Science, 352 (6290), 1206 (2016).

[2] M. S. Kim et al., Phys. Rev. B 77, 144425 (2008).

[3] M. S. Kim et al., Phys. Rev. Lett. 110, 017201 (2013).

[4] M. Mourigal et al., Nat. Phys. 9, 435 (2013).

[5] J. Schlappa et al., Nature 485, 82 (2012). 


\title{
Overview of residual stresses in additive manufactured parts
}

\author{
J. Milner and T. Gnäupel-Herold
}

$\Lambda$ dditive manufacturing (AM) of metals is a process for building parts layer-by-layer that can produce complex geometries that are not achievable by traditional subtractive manufacturing means. However, one of the key barriers to widespread adoption of this process is the build-up of residual stress within the part due to repeated localized melting and solidification during the build process. In this case, residual stress refers to the undesirable internal stress that remains in the part when the AM build process has completed. This stress may lead to unwanted cracks, layer delamination, unexpected failure, warping and lost part tolerances. Therefore, investigation of the residual stress is desired to gain a complete understanding of the influencing factors; such as process parameters, geometry effects, and post-process treatments to better control and mitigate the resultant stress within AM parts.

Simple geometric parts are additively manufactured by the laserbased powder bed fusion process. A recoater blade spreads a thin layer of metal powder over the build plate, where mirrors and lenses direct a laser to selectively melt and fuse the powder corresponding to the cross-sectional layer of the desired part. When the first layer is finished, the build plate is lowered by the thickness of one layer, and the process repeats, building the part layer-by-layer until the part is fully fabricated. This work focuses on parts AM from Inconel 625 and Stainless Steel 17-4 $\mathrm{PH}[1,2]$, which are common materials used in industry. Stress characterization of the AM parts is determined non-destructively by neutron diffraction at the BT8 Residual Stress Diffractometer.

To study geometric effects, a large range of cylindrical parts were designed with various build heights, outer diameters, and inner diameters. A part had either a $6 \mathrm{~mm}$ or $12 \mathrm{~mm}$ outer diameter, was solid or had an inner diameter of $2 \mathrm{~mm}$ and a build height of $10 \mathrm{~mm}, 25 \mathrm{~mm}$, or $40 \mathrm{~mm}$. Figure 1 displays contour stress maps of the AM Inconel 625 cylinders with an outer diameter of $12 \mathrm{~mm}$ and a build height of $40 \mathrm{~mm}$ while comparing the effect of (a) a solid structure versus (b) a structure with an internal diameter of $2 \mathrm{~mm}$. Overall, the parts exhibit large tensile exteriors and compressive interiors regardless of the geometry of the part. Build height of the part, greater than $10 \mathrm{~mm}$, does not affect the stress profile nor its magnitude within identical geometric parts. Comparing the solid cylinders with a $6 \mathrm{~mm}$ and $12 \mathrm{~mm}$ diameters, exhibits a reduction in the magnitude of stress by $\approx 25 \%$ with a corresponding $75 \%$ reduction in cross-sectional area. In addition,
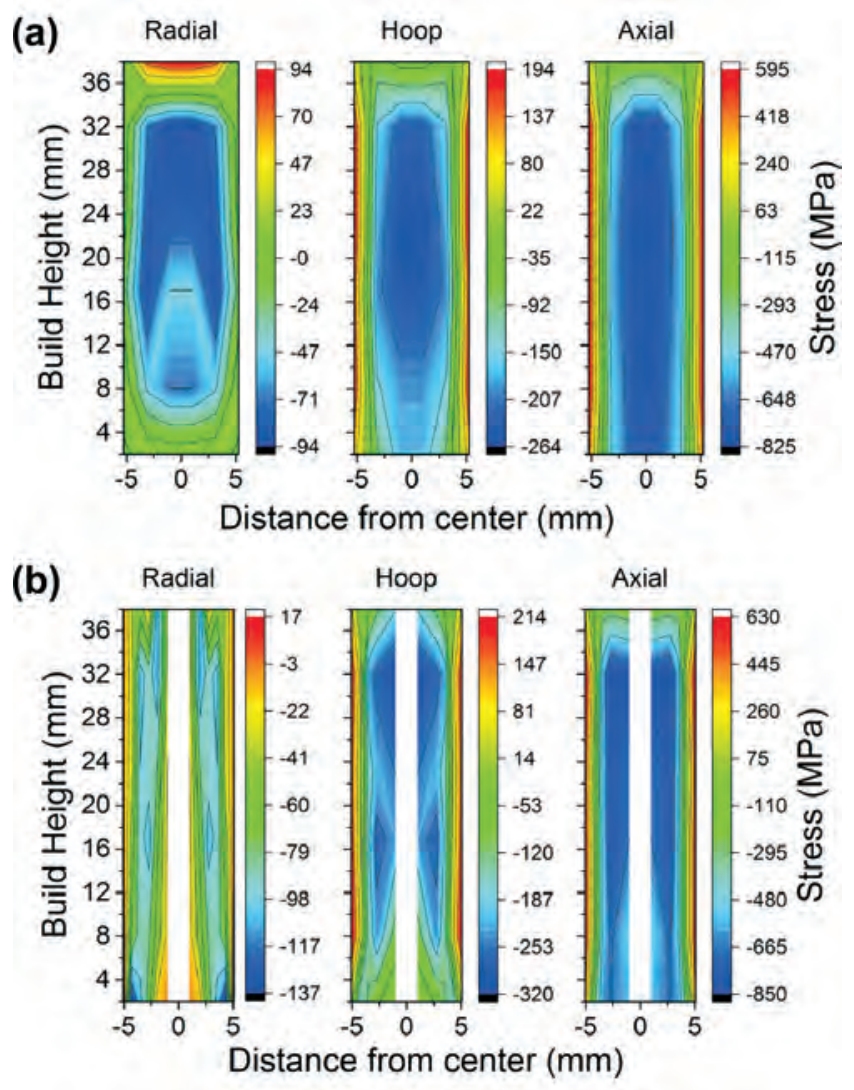

FIGURE 1: Contour stress maps of AM Inconel 625 cylinders with a build height of $40 \mathrm{~mm}$, outer diameter of $12 \mathrm{~mm}$; where (a) is solid and (b) has an inner diameter of $2 \mathrm{~mm}$.

introducing a hole that spans the length of the part does not reduce the overall stresses within the part, as shown in Figure 1. Further examination of the geometric effects still needs to be conducted, with increased spatial resolution of the smaller parts, to quantify the stress profile and magnitude fully.

Increased spatial resolution scans, with a step size of $0.25 \mathrm{~mm}$, were conducted on an AM Stainless Steel 17-4 PH rectangular cuboid (10 mm x $10 \mathrm{~mm} \times 90 \mathrm{~mm}$ ), scan locations indicated in Figure 2(a). Side-to-side scans were performed in both the $x$ and $y$ directions at a build height of $85 \mathrm{~mm}$. The corresponding stress components between the two scan directions were seen to be similar and, thus, averaged into a single representation of 
(a)

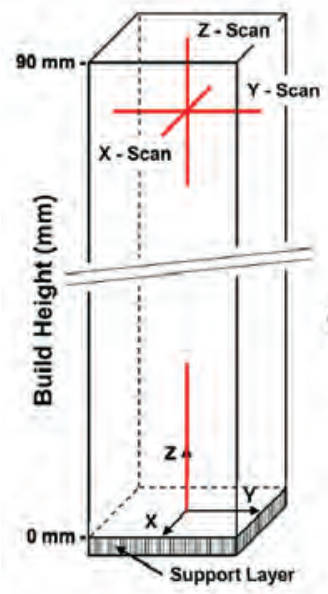

(b)

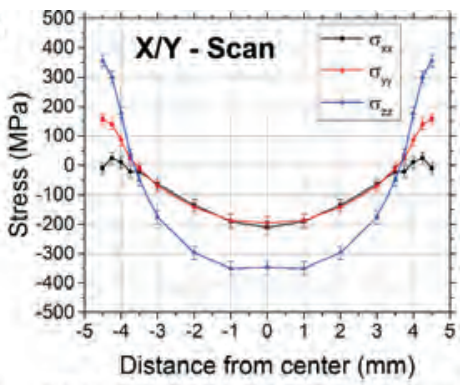

(c)

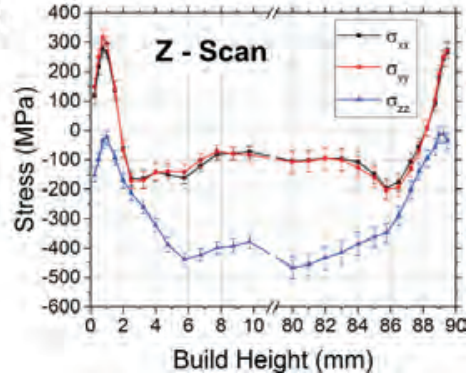

FIGURE 2: (a) Schematic diagram showing the locations of the high-resolution scans of AM Stainless Steel 17-4 PH to quantify the stress profile along the (b) averaged $X$-Scan and $Y$-Scan and (c) Z-Scan above support layer $(0 \mathrm{~mm}$ build height) and near top surface (90 $\mathrm{mm}$ build height).

the three stress components, summarized in Figure 2(b). Another scan was conducted along the $z$-direction portrayed in Figure 2(c); starting above the support layer from $0 \mathrm{~mm}$ to $10 \mathrm{~mm}$ and then continuing at $80 \mathrm{~mm}$ and finishing near the top surface or $90 \mathrm{~mm}$. The stress profiles are seen to differ within the first millimeter from the surfaces; this is caused by the support layer reducing the local stress near the interface. Moreover, the effect of the surface is evident where $\sigma_{x x}$ and $\sigma_{y y}$ exhibit peak stress near the surface and then dropping drastically before stabilizing. The stresses are seen to stabilize beyond $6 \mathrm{~mm}$ from the bottom and top surfaces.

Adjusting the processing parameters does not significantly affect the outcome on the residual stresses of the AM parts; therefore, post-build processing is necessary to reduce the residual stress caused by the build process. This can be done by a thermal treatment process, stress relieving, to the finished AM part. Two identical AM Inconel 625 rectangular cuboids $(10 \mathrm{~mm} x$ $10 \mathrm{~mm} \times 20 \mathrm{~mm}$ ) were prepared and subjected to two thermal treatment procedures: one part was held at $700{ }^{\circ} \mathrm{C}$ for four hours and the other was held at $800^{\circ} \mathrm{C}$ for one hour. Three stress measurement scans were performed on the AM parts along the $x$ and $y$ directions at the mid-level ( $10 \mathrm{~mm}$ from the base) and along the $z$-direction from the base to the top surface. The $x$ and $y$ directional scans were averaged together, due to symmetry, to form a single representation of the stress from side-to-side of the part. The results are summarized in Figure 3, where (a) displays the stresses from side-to-side and (b) the stresses along the $z$-direction. The thermal treatments significantly reduced the magnitude of residual stress, where the $800^{\circ} \mathrm{C}$ procedure was
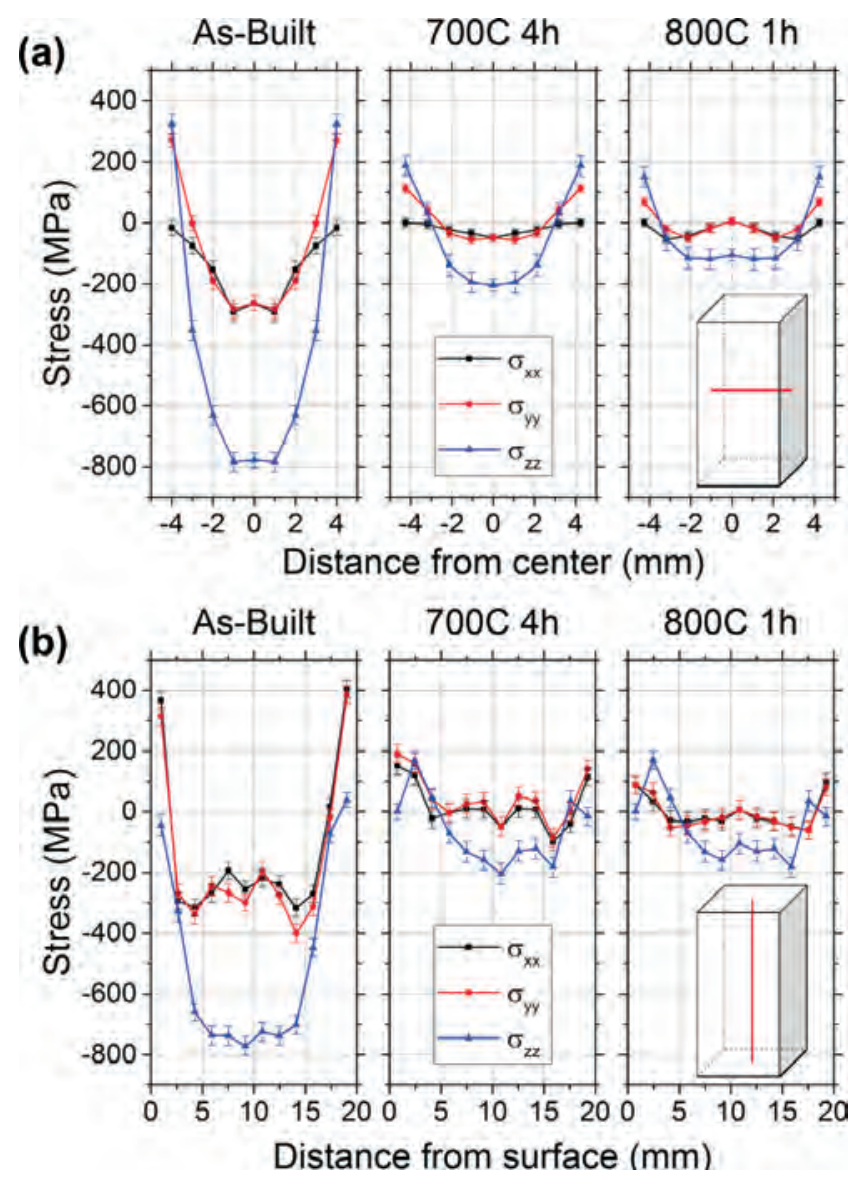

FIGURE 3: Post-build thermal treatment results of an AM Inconel 625 part treated at $700{ }^{\circ} \mathrm{C}$ for four hours and $800^{\circ} \mathrm{C}$ for one hour. Stress profiles of (a) side-to-side scan and (b) build height scan.

slightly more effective at reducing the residual stress. This thermal treatment resulted in an $85 \%$ reduction in peak residual stress of the as-built part.

The resultant residual stresses of AM parts may never be removed completely by optimizing the processing parameters due to the nature of the process itself, as metal powder must be locally melted and cooled repeatedly to form a part. However, the ability to predict the residual stress outcome may lead to controlling the stress magnitude and profile within the part to be less detrimental. Therefore, continued analysis of processing parameters along with geometric effects is needed for a better understanding of the resultant residual stress. Moreover, post-build thermal treatments can be performed to significantly reduce the residual stresses of AM parts.

\section{References}

[1] T. Gnäupel-Herold, J. Slotwinski, S. Moylan, AIP Conf. Proc., 1581, 1205 (2014).

[2] M. Masoomia et al., Data in Brief 13, 408 (2017). 


\title{
Stresses in automotive fastener joints
}

\author{
T. Gnäupel-Herold, ${ }^{1}$ J. L. Milner, ${ }^{1}$ and J. D. Skovron ${ }^{2}$
}

W hether mandated by government regulation or by consumer demand - automotive fuel economy is a topic of imminent importance. Lighter cars and trucks require less energy to accelerate and move - in other words, weight reduction is a straightforward way to improve gas mileage. The single heaviest component of a passenger vehicle is the body structure, and here lies the greatest potential for significantly reducing the vehicle mass - hence the ongoing transition from steel-based structure to bodies composed at least partially of aluminum, magnesium and advanced high strength steels. The latter offers weight savings through high strength which allows lightweighting through dimensional reductions. The introduction of new materials combinations such as nonferrousto-ferrous and nonferrous-to-nonferrous requires methods of joining beyond spot welding of which we examine here three examples: self-piercing rivets (SPR), flow drill screw driving (FDS) and composite friction fasteners (CFF). All three methods are able to join aluminum and steel sheet (FDS: steel needs pilot hole) together. These technologies are capital-intensive and they are currently used on a wide scale (full auto body) in the higherend car segment. The transition to the broader car market has begun, but it will be further aided by more breadth and detail of understanding of joint mechanical properties and behavior.

Data on residual stresses in FDS, SPR and CFF joints are scarce due to the lack of suitable measurement methods other than neutron or synchrotron diffraction which are the only methods that can provide the necessary penetration and spatial resolution $(\approx 1 \mathrm{~mm})$ required for resolving the stress fields with sufficient detail. The

need for more numerical stress data is felt especially in modeling both of long term durability (fatigue) and behavior under peak load such as in crash conditions.

Of the three methods two - FDS and CFF - are thermal processes where sheet penetration is achieved by thermal softening of the aluminum sheet. CFF goes one step further where even more friction heat is built up to achieve fusion to the base steel sheet.

In both cases residual stresses arise from quenching after local heating and joining of materials with different thermal expansion coefficients. In contrast, SPR is non-thermal; residual stresses are produced through displacement of sheet material and through the geometrical constraint from the combined plastic deformation of rivet and sheets. All three processes are shown in schematic in Figure 1.

The residual stress measurements were done using the BT8 stress diffractometer at the NCNR. The results reflect the process differences mentioned before: thermally dominated processes where material is displaced at high temperatures (FDS and (FF) display similar characteristics in stress compared to SPR where a cold material displacement occurs [1]. Here, the term 'displacement' refers to sheet material volume pushed aside by the penetrating fastener. Therefore, material displacement is synonymous with plastic deformation. Locally different levels of plastic deformation are the predominant source of residual stresses. For FDS and CFF tensile stresses dominate within the first $\approx 5 \mathrm{~mm}$ in the sheet outside the fastener after

\section{Light Metal Joining Methods}
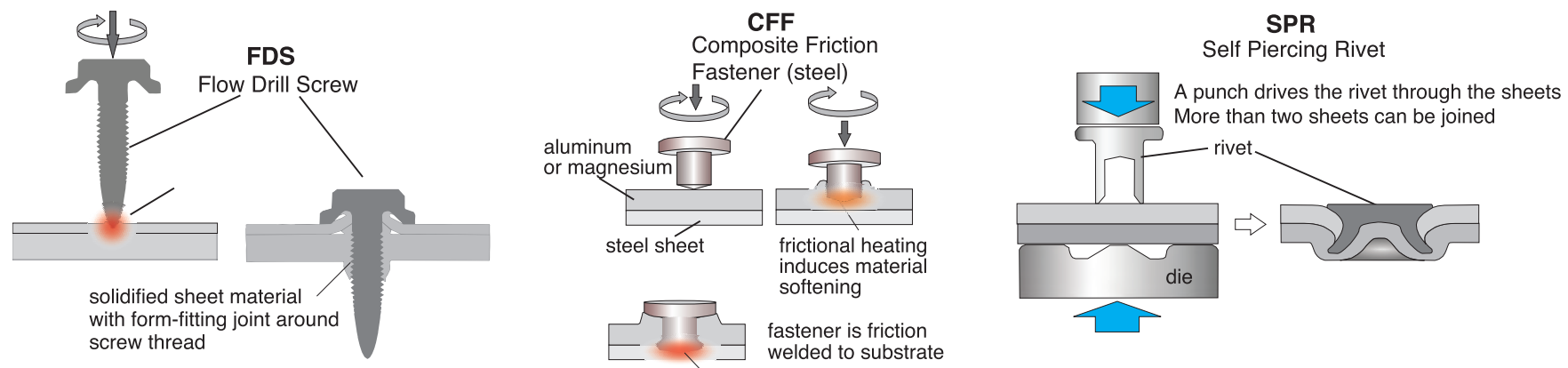

FIGURE 1: Light metal joining methods.

\footnotetext{
${ }^{1}$ NIST Center for Neutron Research, National Institute of Standards and Technology, Gaithersburg, MD 20899

2 Clemson University, Greenville, SC 29634
} 

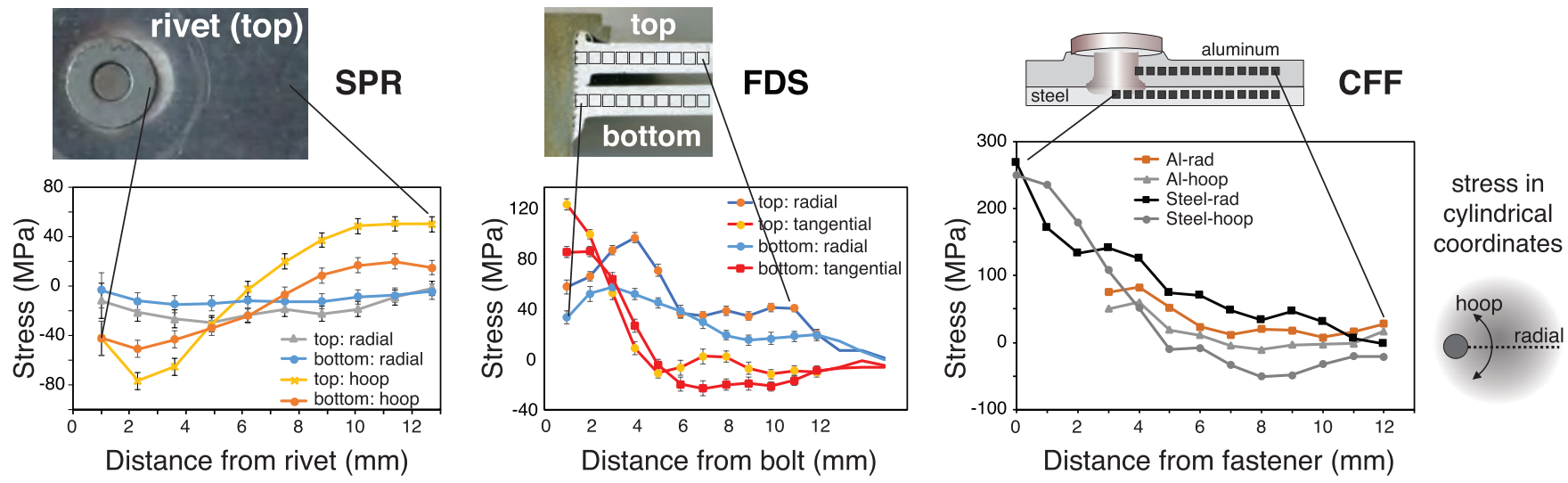

FIGURE 2: Overview of stresses in SPR, FDS and CFF joints.

which stresses turn moderately compressive as a result of stress balance boundary conditions. It is worth noting that stresses in aluminum sheet are slightly higher in FDS compared to CFF. The most likely explanation is that in the CFF joint the higher heat input (steel sheet needs much higher temperatures for fusing with the fastener) and the resulting longer cooling time provides for a more spatially homogeneous deformation during cooling to room temperature. In contrast, stresses in the SPR start out as compressive near the rivet which conforms to expectation for radial stresses. The spatial resolution of the measurement $(\approx 1.5 \mathrm{~mm})$ is insufficient to capture stresses in the aluminum sheet immediately next to the rivet. For this region, tensile hoop stresses are expected, and this expectation is supported by high resolution results published in the literature for steel sheet (note that iron is a much stronger scatterer than aluminum, and it allows for proportionally smaller gage volumes).
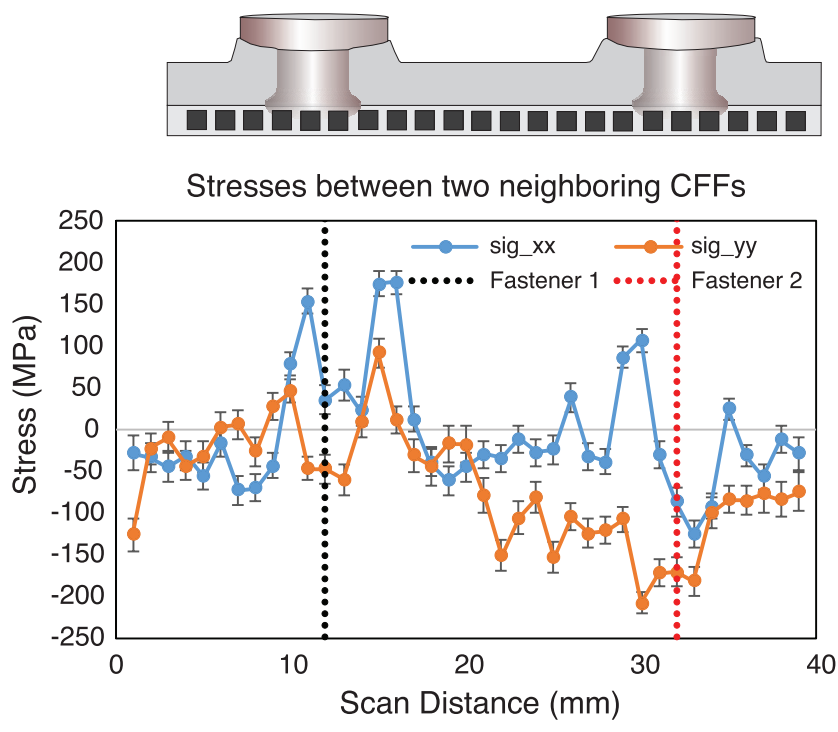

FIGURE 3: Stresses for neighboring CFFs. The $\mathrm{x}$-direction refers to the line connecting the two fasteners. The $y$-direction is perpendicular to this line.
Since spacing of joints depends on sheet thickness (thinner sheet requires reduced distance between joints) it is possible that neighboring joints produce overlapping stress fields. Typical distances range from $10 \mathrm{~mm}$ to $30 \mathrm{~mm}$ which can produce noticeable effects when stress extent up to $10 \mathrm{~mm}$ outside the joint. Figure 3 shows the combined stresses of two CFFs in a 2-sheet composite ( $2 \times$ aluminum $+1 \times$ steel).

There is notable asymmetry in the stresses between the two joints which is most likely the result of the joints produced consecutively, i.e., the first joint had already some time to cool while the second fastener was placed. However, overall cooling time is extended due to nearly twice the heat input over a longer time frame and over a larger area. Thus, thermal gradients are lower compared to the single CFF joint, and so are the residual stress (see Figure 2, right and Figure 3). This result in particular highlights the need for stress measurements and specimens that closely mirror conditions found production sheet joints.

\section{Reference}

[1] J. Milner, T. Gnäupel-Herold, J. D. Skovron, Proceeding of MSEC2016, Blacksburg, VA, 6/27-7/1, MSEC2016-8823. 


\title{
Holography with a neutron interferometer
}

\author{
D. Sarenac, ${ }^{1,2}$ \\ D. A. Pushin, ${ }^{1,2}$ D. G. Cory, ${ }^{2,3,4,5}$ \\ C. B. Shahi, ${ }^{6,7}$ \\ B. Heacock, $, 8,9$ \\ C. W. Clark, $, 6,10$ \\ M. Arif, ${ }^{10}$ and M. G. Huber ${ }^{10}$
}

W e have used neutron beams to create holograms of large solid objects [1], revealing details about their interiors in ways that ordinary laser light-based visual holograms cannot. Holography was introduced by Gabor in 1948 [2], who showed that a far-field electron micrograph of an object could be used to make a transmission mask that allows the object to be reconstructed with visible light. The advent of coherent laser light sources in the 1960s made all-optical holography practical, to a degree that optical security holograms are now routinely printed on many paper currencies, credit cards, and identification documents [4]. Holography remains a vibrant field of research in imaging science. Our method is a simple adaptation of the two-beam wedge technique introduced by Leith and Upatnieks [3], and we discuss it using the conventional terminology of object, reference, and reconstruction beams.

Our object is a spiral phase plate (SPP) [5]. Our neutron holograms resemble the fork dislocation gratings which have been used to transfer angular momentum to atoms, electrons, and light [6]. Digital reconstruction of this hologram provides information about the phase generated by the SPP.

As shown in Fig. 1, our object beam consists of neutrons that have passed through an aluminum SPP in one arm of a perfect silicon neutron interferometer (NI). The SPP imprints a spatiallyvarying phase of $q \varphi$ upon the neutron input beam, where $q$ is the topological charge of the SPP and $\varphi$ is the azimuthal angle, with coordinate origin being the center of the SPP surface. The other arm of the NI provides a reference beam. The holographic image is constructed from the interference of the object and reference beams, as recorded by a neutron sensitive digital camera. When no optical device is present in the reference arm, this interferogram displays the topological charge of the object beam, which is the same as that of a beam with orbital angular momentum (OAM) of $q \hbar$. By placing a wedge into the reference beam, we introduce a linear gradient which effectively tilts the neutron wave fronts.

a)

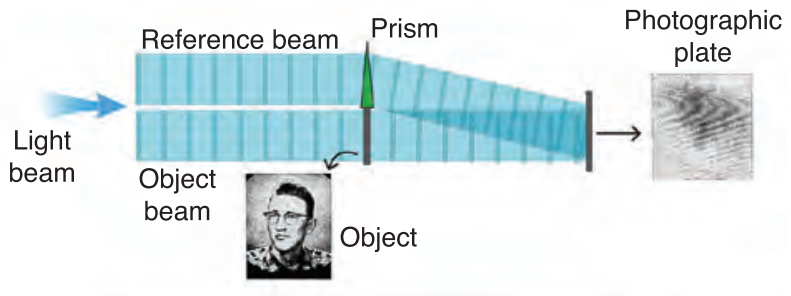

b)

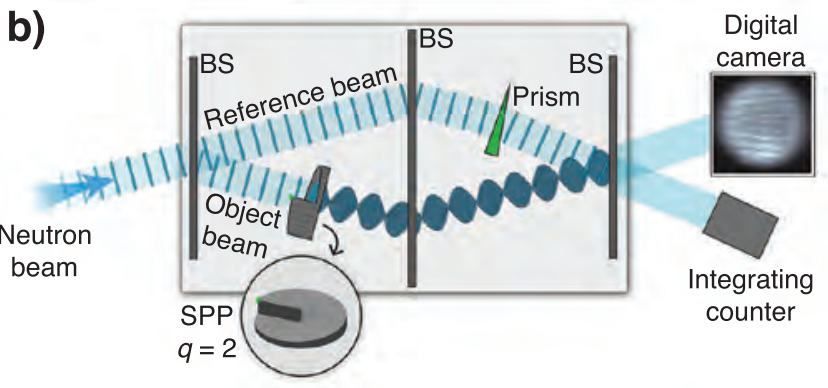

FIGURE 1: (a) The off-axis method of optical holography of semitransparent objects introduced by Leith and Upatnieks. (b) An artistic depiction of the neutron holography experiment.

Incident on the $\mathrm{Nl}$ are monoenergetic neutrons with energies around $11 \mathrm{meV}$, corresponding to a wavelength of $\lambda=0.271 \mathrm{~nm}$. The $\mathrm{NI}$ was made using a single ingot of silicon machined so that it has three blades supported by a common base. The common base insures sub-arcsecond alignment between the crystal blades. Neutrons entering the interferometer are Bragg diffracted by the (111) lattice planes of the crystal blades forming two spatially separate paths. The last NI blade coherently combines the two paths and the intensity at the camera is then given by:

$$
I=A+B \cos \left(\vartheta_{\text {wedge }}-q \varphi+\theta\right),
$$

where $A$ and $B$ are experimental constants, $\vartheta_{\text {wedge }}$ is the action of the wedge, and $\theta$ is the inherent different between the reference 


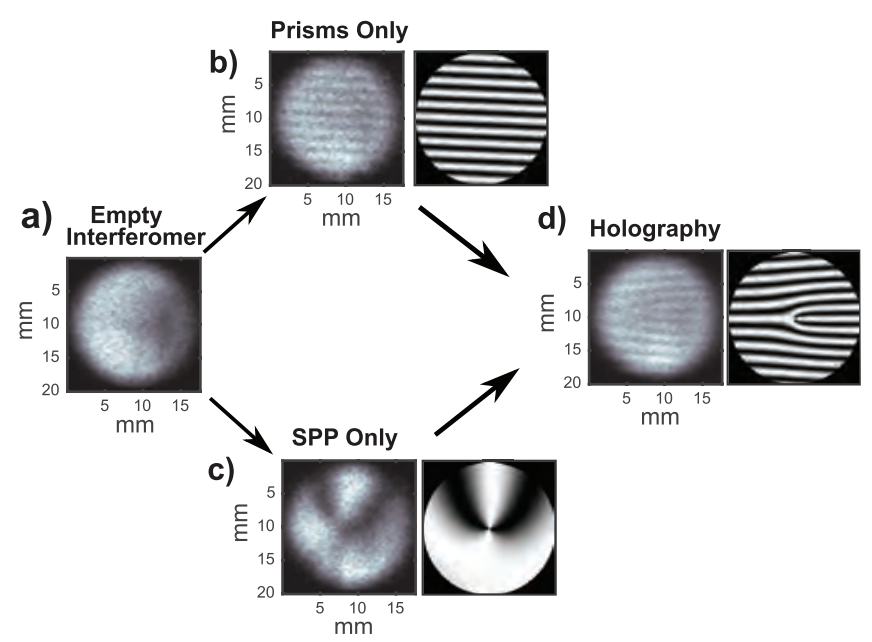

FIGURE 2: Measured and simulated interferograms at the 2D detectors for the $q=2 \mathrm{SPP}$ and $\mathrm{a} 6^{\circ}$ fused silica wedge shown independently as well as together.

and object paths. This interference is then detected using either a fully integrating ${ }^{3} \mathrm{He}$ proportional counter or a neutron-sensitive imaging camera.

An aluminum SPP with $q=2$ was placed in the object beam. The SPP was made with a standard milling machine cutting a spiral staircase into the surface of a segment of aluminum dowel. A topological charge $q=2$ is obtained with a staircase with a total vertical descent of $224 \mu \mathrm{m}$, which is $\approx 10^{6} \times \lambda$. Those experienced in optical design for visible light may find it surprising that the mechanical figure of an optical component need only be controlled within a few thousand multiples of the operational wavelength. This is possible with neutrons, since neutron indices of refraction for most materials differ from unity by a few parts per million. In the reference beam a vertical linear gradient was introduced by using two identical fused silica optical wedges arranged back-to-back. These wedges had a $6^{\circ}$ angle and each could be rotated independently a full rotation of $2 \pi$.

In our experiments, the integrating counter was used to measure the average rate of neutrons exiting the interferometer of $r \approx 20 / \mathrm{s}$. This rate determines an average time interval $\tau=1 / r=50$ ms between detection of successive neutrons. Since the distance of the $\mathrm{NI}$ from the reactor is $30 \mathrm{~m}$, and the neutron velocity is $1,460 \mathrm{~m} / \mathrm{s}$ it takes $20 \mathrm{~ms}$ for a neutron to travel from the reactor to the NI. Thus, just after one neutron has been detected, the next neutron to be detected has not yet been produced by the reactor. The interference fringes that are seen in our data are truly those of neutrons interfering with themselves.
Reactor fluctuations were monitored using the ${ }^{3} \mathrm{He}$ detector. The interferograms were recorded on a neutron-sensitive digital camera that has an active area of $25 \mathrm{~mm}$ diameter and a spatial resolution of $100 \mu \mathrm{m}$. The neutron quantum efficiency of the camera is $18 \%$ and individual images were taken in 28-hour runs.

Figure 2 depicts interferograms for four different configurations. Figure $2 \mathrm{a}$ shows the grayscale intensity profile obtained with an empty interferometer. Figure $2 \mathrm{~b}$ shows the experimental and simulated interferograms for the case in which only the wedge is placed in the reference beam of the interferometer. The number of fringes in the simulated interferogram agree with the measured image. Figure 2c shows the interferogram when only the SPP is present in the object beam. The expected fork grating pattern is recognizable in this hologram.

Using a neutron interferometer, we have for the first time demonstrated digitally recorded neutron holography of a macroscopic object: a spiral phase plate. Numerical reconstruction of the recorded hologram reveals the variation of neutron phase over the surface of that plate. This work adds to the palette of techniques scientists have to explore the internal structure of solid materials. This method provides a new tool for interferometric testing of neutron optics and the characterization of coherence of neutron beams. It offers an extension of coherent phase control techniques to applications in neutron radiography and imaging, which are uniquely useful in the analysis of buried interfaces [7].

\section{References}

[1] D. Sarenac et al., Optics Express 24, 22528 (2016).

[2] D. Gabor, Nature 161, 777 (1948).

[3] E. N. Leith, J. Upatnieks, J. Opt. Soc. Am. 53(12), 1377 (1963).

[4] S. F. Johnston, Holograms: A Cultural History, Oxford University, (2016).

[5] C. W. Clark et al., Nature 525, 504 (2015).

[6] A. Yao, M. Padgett, Adv. Opt. Photon. 3, 161 (2011).

[7] J. D. Fairweather et al., J. Electrochem. Soc. 160, F980 (2013). 


\section{aCORN: A precision measurement of the electron-antineutrino angular correlation in free neutron beta decay}

M. T. Hassan, ${ }^{1,2,3}$ W. A. Byron, ${ }^{2}$ B. Collett, ${ }^{4}$ G. Darius, ${ }^{2}$ C. DeAngelis, ${ }^{2}$ S. Dewey, ${ }^{1}$ G. Jones, ${ }^{4}$ A. Komives, ${ }^{5}$ M. P. Mendenhall, ${ }^{1}$ J. Nico, ${ }^{1}$ E. Stephenson, ${ }^{6}$ F. E Wietfeldt, ${ }^{2}$ and B. G. Yerozolimsky ${ }^{7}$

$\square$ he angular correlation between the beta electron and antineutrino in nuclear beta decay is characterized by the dimensionless parameter " $a$ ". In the neutron, it has the largest uncertainty (about $5 \%$ ) among the group of neutron decay correlations that have been seriously experimentally determined. In the Standard Model of Particle Physics, the axial vector $\left(\mathrm{G}_{\mathrm{A}}\right)$ and vector $\left(\mathrm{G}_{\mathrm{V}}\right)$ coupling constants of the weak interaction can be obtained from the angular correlations in free neutron beta decay and the neutron lifetime. A substantial reduction in the uncertainty of the " $a$ " coefficient will give an improved value of the ratio of $\mathrm{G}_{\mathrm{A}}$ and $\mathrm{G}_{\mathrm{V}}$ and improved limits on non-Standard Model scalar and tensor weak currents. In this report, we present the most precise measurement of the neutron " $a$ " coefficient reported to date.

A novel technique first proposed by Yerozolimsky and Mostovoy was implemented in the aCORN experiment to measure the " $a$ " coefficient where an asymmetry in proton-electron time-of-flight was constructed that directly yields " $a$ " without needing precise proton spectroscopy. The neutron decay region was on the axis of a uniform magnetic field generated by a solenoid [1]. The beta electrons and recoil protons were detected at the two ends of the solenoid. The third decay particle, the antineutrino, was not detected directly. Constraints coming from conservation of energy and momentum allowed two groups of antineutrino momenta to be isolated. One group traveled in the same hemisphere as the electron while the other group traveled in the opposite hemisphere. These two groups were distinguished by proton time-of-flight in the beta energy range: a fast group and a slow group. The asymmetry in proton counts between the fast and slow groups was proportional to the " $a$ " coefficient.

A novel backscatter suppressed beta spectrometer was designed and built for beta energy detection [2]. The energy of the electrons was detected by a plastic scintillator slab. An array of eight plastic scintillators was assembled in a cone shaped arrangement to veto the backscattered electrons. A $600 \mathrm{~mm}^{2}$ silicon surface barrier detector with a set of focusing electrodes was used to detect protons. The proton detector was positioned slightly

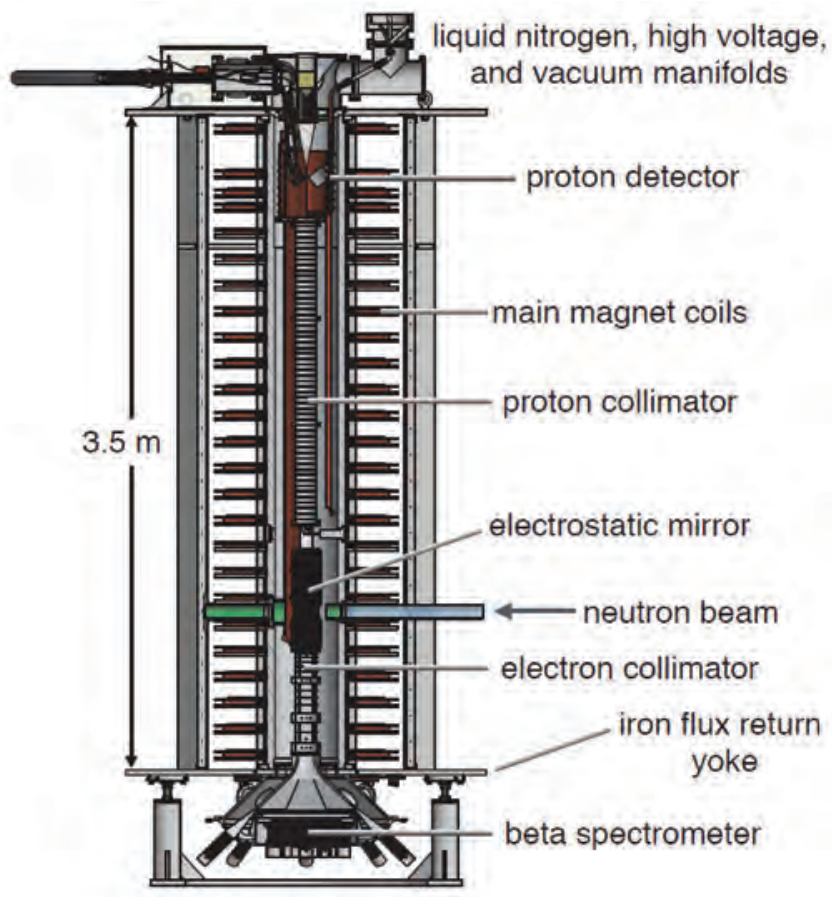

FIGURE 1: Schematic diagram of the aCORN apparatus.

off-axis to eliminate electron backscattering from its surface to the beta spectrometer. A set of 24 water-cooled pancake coils powered in series was used to generate a $36.2 \mathrm{mT}$ axial magnetic field. Each pancake coil had a circular axial trim coil. There were 25 pairs of rectangular trim coils in each direction to eliminate transverse fields which can lead to a false asymmetry on proton counting. Electron and proton collimators were mounted on the same axis as the magnetic field to limit the transverse momenta of decay particles. An electrostatic mirror was installed around the decay region with $3 \mathrm{kV}$ potential to reflect and pre-accelerate all protons toward the proton detector. A more detailed description of the apparatus is presented in reference [3]. Figure 1 shows the schematic diagram of the aCORN apparatus. 


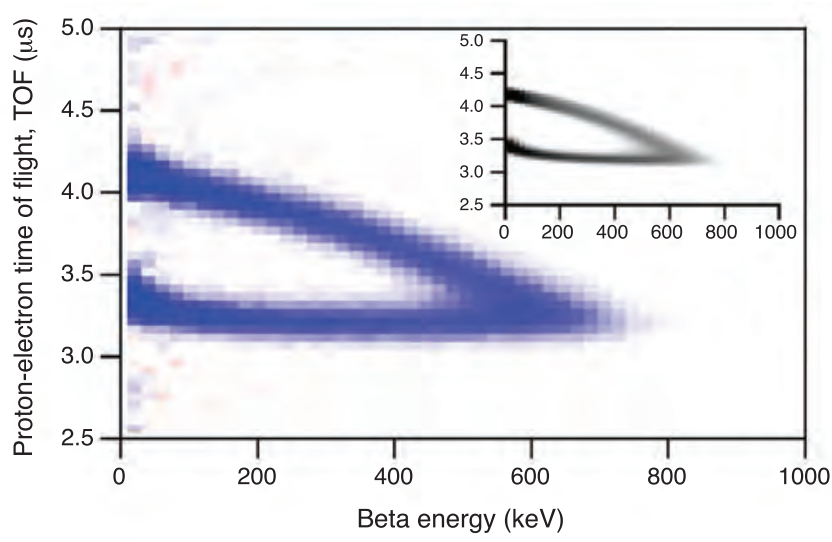

FIGURE 2: A typical background subtracted, veto suppressed and energy calibrated "wishbone" histogram from a dataset of about 400 beam hours. Blue pixels represent positive numbers and red pixels represent negative numbers (due to background subtraction). The lower branch (fast group) in the plot contains protons associated with the antineutrinos traveling in the same hemisphere as the electrons and the upper branch (slow group) contains protons associated with antineutron and electron momenta in opposite hemispheres. Beta energy slices were taken from the plot and wishbone asymmetry was calculated from number of protons in these two groups for each slice. Inset: Monte Carlo simulation.

The aCORN experiment was installed and operated the NIST Center for Neutron Research (NCNR). The experiment was run in two phases. In the first phase, the apparatus was on the NG-6 beamline and data acquisition was done with a goal of $4 \%$ relative uncertainty. The experiment was moved to the NG-C beamline for the second phase with a goal of $1 \%$ relative uncertainty. Analysis of the dataset collected in the first phase, totaling 1900 beam hours, has been completed. Figure 2 shows a background subtracted "wishbone" histogram plot of proton time-of-flight vs beta energy plot from a dataset of about 400 beam hours. The details of the analysis procedure are given in references $[3,4]$. The measured value of the " $a$ " correlation was $-0.1090 \pm 0.0041$, a relative uncertainty of $3.8 \%$ [4]. The comparison between " $a$ " coefficient measured by different experiments is shown in Figure 3.

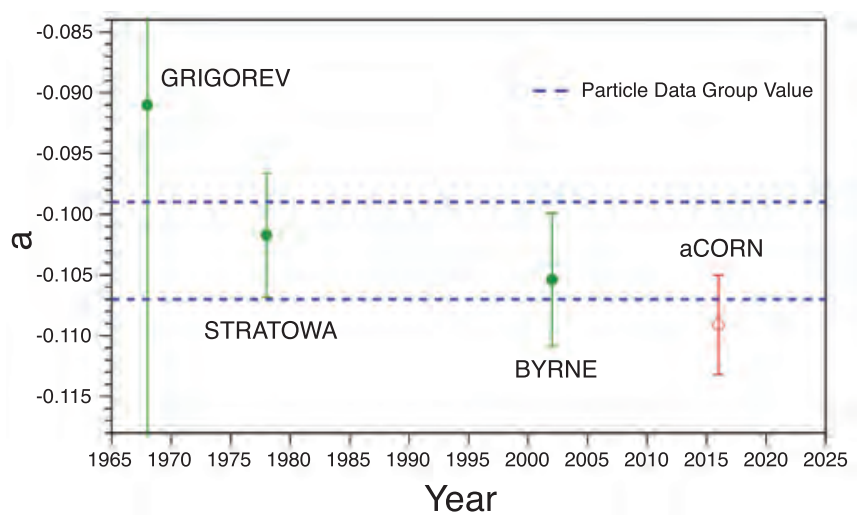

FIGURE 3: A comparison between measured "a" coefficient by different experiments.

In conclusion, we have reported the most precise measurement of the electron-antineutrino angular correlation in free neutron beta decay from the first phase of the aCORN experiment. The statistical uncertainty was the largest uncertainty. More data were taken on a higher flux NG-C beamline with several instrumentation upgrades in the second phase of the experiment. The wishbone rate was 4.6 times higher at NG-C than on NG-6. A faster DAQ was designed to handle the higher rate. The most notable upgrade was the redesigned improved electrostatic mirror. The magnetic field was also significantly reconfigured to obtain more uniform magnetic field near the proton detector. We believe, after analyzing the data from the second phase, we will be able to achieve a final uncertainty of about $1 \%$ in the " $a$ " coefficient.

\section{References}

[1] B. G. Yerozolimsky, et al., arXiv:nucl-ex/0401014 (2004).

[2] M.T. Hassan et al., Nucl. Instr. Meth. A, 867, 51 (2017).

[3] B. Collett et al., Rev. Sci. Instrum. (2017) in press, arXiv:1701.05184.

[4] G. Darius et al., Phys. Rev. Lett 119, 042502 (2017). 


\title{
Conformational studies of virus/polymer conjugates and their applications in medicine
}

\author{
P. W. Lee, ${ }^{1}$ S. A. Isarov, ${ }^{1}$ J. D. Wallat, ${ }^{1}$ S. K. Molugu, ${ }^{1}$ S. Shukla, ${ }^{1}$ J. E. P. Sun, ${ }^{1}$ J. Zhang, ${ }^{1}$ Y. Zheng, ${ }^{1}$ \\ M. L. Dougherty, ${ }^{2}$ D. Konkolewicz, ${ }^{2}$ P. L. Stewart, ${ }^{1}$ N. F. Steinmetz, ${ }^{1}$ M. J. A. Hore, ${ }^{1}$ and J. K. Pokorski ${ }^{1}$
}

$\mathrm{N}$ anotechnology has revolutionized the way that research and development are being conducted in the pharmaceutical field. Nanomedicine, the use of nanoparticles to deliver drugs or imaging agents, has the promise to greatly change patient outcomes. Nanoparticle loaded therapies can better target sites of disease like cancer or cardiovascular disease, and eliminate the toxic side-effects of traditional small-molecule chemotherapies. One of the critical limiting factors of nanomedicine, however, is the body's innate ability to recognize foreign particles and rapidly clear these nanotherapies through immune-mediated mechanisms. Regardless of the material that the nanoparticle is derived from, whether it be inorganic, organic, or bio-derived, particles in the nanoscale size regime quickly elicit trafficking to the liver, lungs, or kidneys where they are eliminated from the body.

Over the past several decades, researchers have found ways to mitigate this immune clearance to enhance circulation lifetime and hence better target the delivery agent to sites of disease. The "gold standard" for about 40 years has been the covalent attachment of poly(ethylene glycol) (PEG), also termed PEGylation, to a particle surface. This hydrophilic polymer creates a highly hydrated layer that blocks biological macromolecules from recognizing the surface of the particles to which PEG is attached. Due to the ubiquitous use of PEG in pharmaceuticals and consumer products, though, a growing subset of the population has developed anti-PEG antibodies rendering PEGylation ineffective. Since the inception of PEGylation, polymer chemistry has advanced to allow for synthesis of tightly controlled polymers with diverse function that evade anti-PEG antibodies. For instance, acidic stability, immunological shielding, and catalytic function have all been incorporated into water soluble polymers. Even more importantly, these types of polymers show improved immune evasion and stabilization of foreign particles in vivo. Early studies have shown that the polymer conformation is critical in dictating biological activity. For instance, when individual polymer chains interact with each other and with their PEGylated substrate, they block large molecules like antibodies from recognizing the cargo.

We have recently developed new chemistry to attach polynorbornene (PNB) polymers to proteins and have demonstrated biocompatibility. In this study, we elucidated the conformation of several different biologically relevant polymers
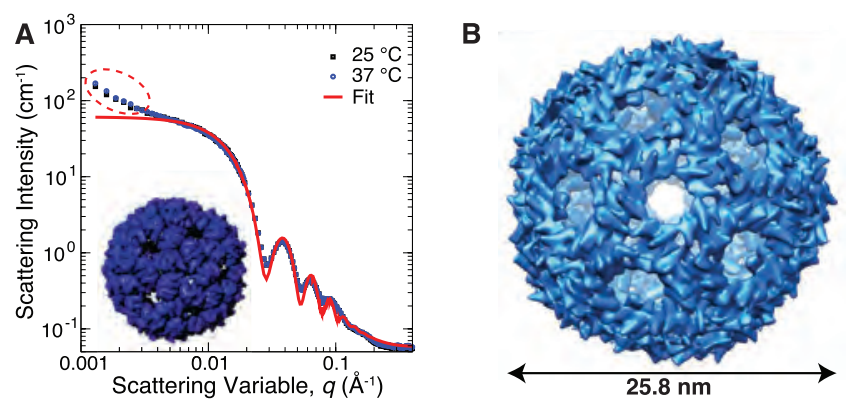

FIGURE 1: (a) SANS from $Q \beta$ prior to conjugation with polymers. (b) $Q \beta$ structure reconstructed from cryo-EM imaging. The circled region in (a) implies a small degree of aggregation of particles in solution.

when attached to a virus-like particle derived from bacteriophage $\mathrm{Q} \beta$ using small-angle neutron scattering (SANS) [1]. $Q \beta$ is a homogeneous biologically-derived nanoparticle that has been extensively used for drug delivery, molecular imaging, and immunotherapy. The precise placement of functional groups, ability to modify the particle genetically, and particle homogeneity make this an incredibly powerful platform for nanomedicine and provided impetus to understand the conformation of these polymers when arrayed multivalently about the surface.

SANS measurements were performed on native $\mathrm{Q} \beta$, as well as $Q \beta$ conjugated with $P E G$, poly(oligo(ethylene glycol) methyl ether acrylate) (POEGMEA), and PNB to determine the conformation of the grafted polymers. SANS intensities for native $Q \beta$ are shown in Figure $1 \mathrm{a}$ at $20^{\circ} \mathrm{C}$ (black) and $37^{\circ} \mathrm{C}$ (blue), demonstrating that the structure of the bare nanoparticle does not vary with temperature. The red dashed circle shows an upturn at low values of the scattering vector $q$, implying the presence of a small amount of aggregation in the sample. Fits to the scattering intensity using a spherical core-shell form factor determined the nanoparticle is a hollow shell approximately $1.4 \mathrm{~nm}$ thick, and with an overall diameter of approximately $25.8 \mathrm{~nm}$, in good agreement with previous studies. Three dimensional reconstructions of the capsid from cryogenic electron microscopy (cryo-EM) tomography, shown in Figure 1b, confirm the structure that was determined from SANS.

Upon attaching polymers to the surface of $\mathrm{Q} \beta$ particles, the scattering intensities (Figure 2) exhibited several distinct

\footnotetext{
${ }^{1}$ Case Western Reserve University, Cleveland, $\mathrm{OH} 44106$

2 Miami University, Oxford, $0 \mathrm{H} 45056$
} 

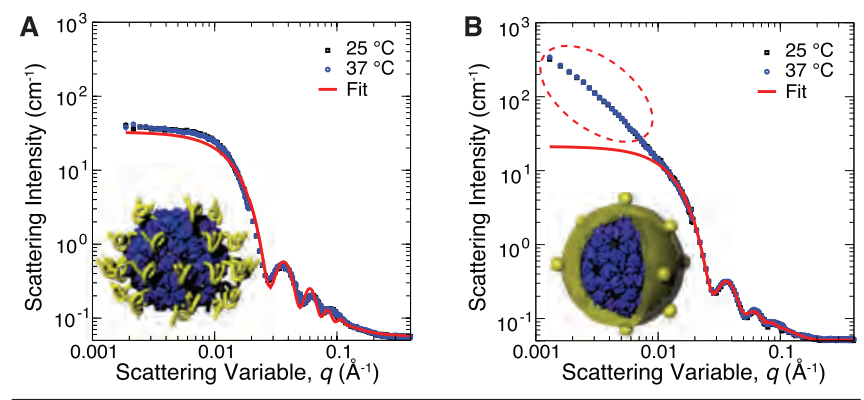

FIGURE 2: SANS measurements of $Q \beta$ grafted with (a) PEG and (b) POEGMEA at $25^{\circ} \mathrm{C}$ and $37^{\circ} \mathrm{C}$. No discernable structural changes occur as temperature is varied.

changes. After attaching PEG to the particle surface, the small upturn observed for native $\mathrm{Q} \beta$ disappeared, indicating that PEG enhances the dispersion of $Q \beta$ in solution (Figure 2a). The scattering intensity was fit using a core-shell-chain form factor [2], and revealed that the nanoparticle was grafted with approximately 80 chains which had an average radius of gyration of $R_{\mathrm{g}} \approx 3.7 \mathrm{~nm}$. No temperature dependence was observed. The SANS data also indicated that the conformation of the PEG chains was slightly swollen (i.e., $R_{\mathrm{g}} \sim N^{0.53}$ ), implying favorable interactions between the polymers and the solvent. For $\mathrm{Q} \beta$ particles that were conjugated with POEGMEA (Figure 2b), a large upturn in the scattering intensity (dotted circle) appears at low $q$, indicating substantial aggregation of the nanoparticles, and confirmed by dynamic light scattering (DLS). Interestingly, fits of the SANS intensities using the core-shell-chain model indicated the presence of approximately 26 compact objects on the surface (i.e., a raspberry-like structure), which were later determined to be polymer globules with a radius of approximately $2 \mathrm{~nm}$. The measured shell thickness of the particle increased from $1.4 \mathrm{~nm}$ to $3.6 \mathrm{~nm}$, demonstrating that the polymer is forming a surface layer as well. SANS measurements of Q $\beta$-PNB displayed the largest differences in the scattering pattern (Figure $3 a$ ). At $25^{\circ} \mathrm{C}$, the particles are well-dispersed in the solution, and the scattering can be fit to a "hollow raspberry" model [1], consisting of a number of spheres grafted to the surface of a larger, hollow sphere. The fits determined that approximately 127 PNB chains were attached to the $\mathrm{Q} \beta$ particles, each forming a small sphere with an average radius of $1.7 \mathrm{~nm}$. Similar to the Q $\beta$-POEGMEA particles, the grafted chains also formed a surface layer $4.2 \mathrm{~nm}$ in thickness significantly increasing the total thickness of the particle. At $37^{\circ} \mathrm{C}$, the scattering profile became dominated by a large tail at low values of $q$, implying that some aggregation of the particles occurred. A reconstruction of the particle structure from cryo-EM imaging is shown in Figure $3 \mathrm{~b}$, and is in excellent agreement with the SANS measurements.

After confirming the conformational attributes of the various polymer conjugated particles, we sought to assess biological function. A key component of polymer coating is the ability for
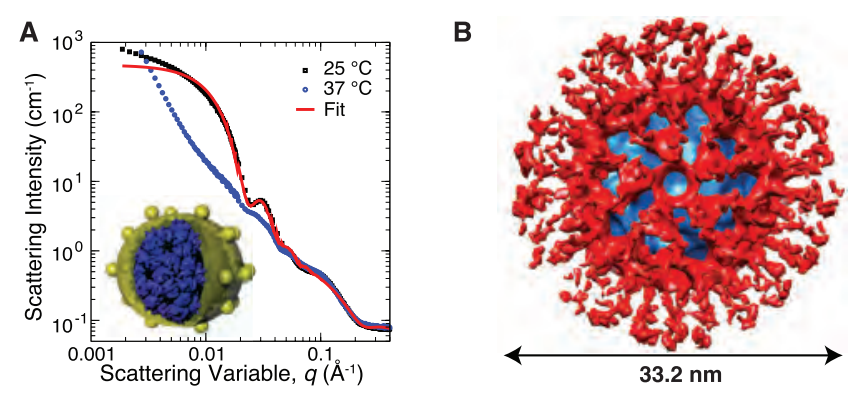

FIGURE 3: (a) SANS from Q $\beta$-PNB, showing the distinct pattern characteristic of a sphere with polymer globules on the surface. (b) Structure of $Q \beta-P N B$ determined from cryo-EM.

particles to evade immune-mediated clearance. More specifically, coated particles should not be recognized by carrier-specific antibodies. In our study, we utilized serum highly enriched in anti-Q $\beta$ Immunoglobulin- $\mathrm{G}(\mathrm{IgG})$ and evaluated the ability of this serum to bind to the polymer coated particles, when compared to unmodified $Q \beta$. Not surprisingly, the $Q \beta-P E G$ conjugate diminished antibody recognition by $\approx 50 \%$, as would be expected given PEG's ubiquitous use. Q $\beta$-POEGMA, by contrast, only showed a moderate decrease in antibody recognition. The best performing polymer coating was Q $\beta-P N B$, further diminishing antibody binding by $\approx 15 \%$ when compared to $Q \beta$-PEG. These results imply a very different mechanism of immune shielding, given the vastly different conformations of the polymers attached. Since POEGMA and PNB have similar conformations, it can be inferred that grafting density is an equally important feature, since PNB had a significantly higher particle coverage. These studies further underscore the complex nature of the biological response and point toward a combination of conjugation chemistry along with polymer conformation as being important factors to consider when selecting polymers for immune shielding.

The results of this study were the first to directly measure and image polymer coated viral nanoparticles. Given the rapidly expanding use of viruses in nanomedicine, this fundamental study opens the door for researchers to dial in specific polymer properties when aiming to install immune shielding function. As the field continues to grow, we expect that these results will have great implications in the development of the next generation of stealth coatings in nanomedicine.

\section{References}

[1] P. W. Lee, S. A. Isorov, J. D. Wallat, S. K. Molugu, S. Shukla, J. E. P. Sun, J. Zhang, Y. Zheng, M. L. Dougherty, D. Konkolewicz, P. L. Stewart, N. F. Steinmetz, M. J. A. Hore, and J. K. Pokorski, J. Am. Chem. Soc. 139, 3312 (2017).

[2] M. J. A. Hore, J. Ford, K. Ohno, R. J. Composto, and B. Hammouda, Macromolecules 46, 9341 (2013). 


\title{
Tuning the morphology of complex oil-in-water- in-oil nanoemulsions using frustrated spontaneous curvature in surfactant mixtures
}

\author{
M. Zhang, P. Malo de Molina, A.V. Bayles, and M. E. Helgeson
}

$\mathrm{T}$ he development of complex emulsions (i.e., multi-phase liquid droplets) for the encapsulation and release of various molecules would allow their use as solution phase reactors to synthesize colloidal particles with complex morphologies for uses such as pharmaceuticals and foods [1, 2]. However, many applications (e.g., nanoparticle drug delivery or catalysis) require the use of nanoscale structures, creating a need for complex nanoemulsions with sizes $<200 \mathrm{~nm}$. Although numerous methods exist to produce micron-scale complex emulsions, there have been few if any reports of similar complex nanoemulsions due to the large energies required for their formation, as well as a lack of strategies for controllably obtaining complex morphologies. This challenge is further exacerbated by the difficulty in characterizing the detailed structure and interactions of nanoemulsions [3].

To overcome these challenges, we have employed properties of surfactant self-assembly to produce and stabilize complex nanoemulsions, and have used a combination of small-angle neutron scattering (SANS) and cryo-transmission electron microscopy (cryo-TEM) to determine their internal structure. Specifically, the use of co-surfactants with opposite spontaneous curvature can produce ultra-low interfacial tension [4], and at the same time influence the preferred interfacial curvature of the oil-water interface.

We tested this hypothesis by using ultrasonication to emulsify a model system of water at various volume fractions ( $\varphi_{w}=0$ to 0.10 ) into cyclohexane containing $30 \mathrm{mM}$ Tween 20 and $50 \mathrm{mM}$ Span 80. SANS was crucial to investigate whether complex nanoemulsions were formed because it not only enables accurate quantification of the internal droplet morphology, but contrast variation by deuteration of the liquid phases allows for selective localization of chemical species to various phases within the internal microstructure. Cryo-transmission electron microscopy (cryo-TEM) was subsequently used to qualitatively confirm the morphologies identified by SANS.

SANS reveals a rich evolution of distinct morphologies that are selected by the water volume fraction, $\varphi_{w}$ (Figure 1a). The data for surfactants in cyclohexane $\left(\varphi_{w}=0\right)$ are described well by ellipsoid structures, indicating the formation of mixed ellipsoidal micelles. At $\varphi_{w}=0.01$, we observe core-shell structures corresponding to a filled water core and surfactant shell that

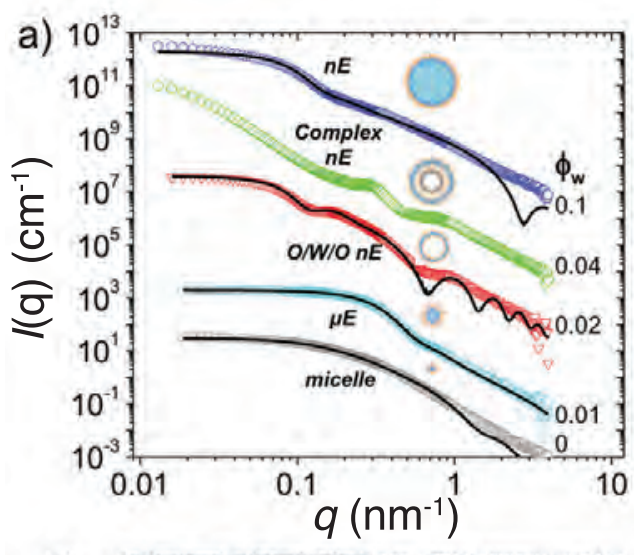

b)

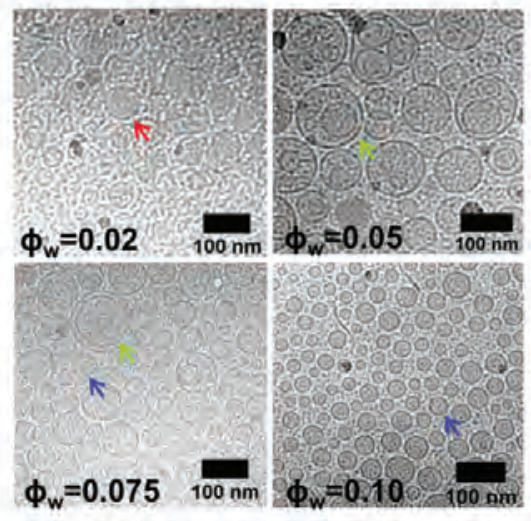

FIGURE 1: a) SANS spectra of samples (30mM Tween 20: 50mM Span80) with h-surfactant $/ \mathrm{D}_{2} \mathrm{O} / \mathrm{d}_{12}$-cyclohexane with increasing water volume fraction $\left(\varphi_{w}=0\right.$, $0.01,0.02,0.04,0.1$ ) show the formation of different microstructures (inset cartoons). b) Cryo-TEM images at different $\varphi_{w}$ (arrows indicate microstructures of the corresponding SANS fits).

indicate the formation of microemulsions. As we increase the water content to $\varphi_{w}=0.02$, we begin to see a $q^{-2}$ decay in scattering intensity at low $q$-values as well as a shoulder at $q \approx 0.9 \mathrm{~nm}^{-1}$, which is indicative of the formation of reverse swollen vesicles, with the shoulder corresponding to distances between surfactant layers. For $\varphi_{w}=0.04$, the characteristic shoulder at $q \approx 0.9 \mathrm{~nm}^{-1}$ is still retained.

To confirm that the high- $q$ shoulder at $q \approx 0.9 \mathrm{~nm}^{-1}$ results from correlations between adjacent surfactant layers, contrast variation measurements were performed at two different contrasts for three water volume fractions $\left(\varphi_{w}=0.01,0.04,0.075\right)$ (Figure 2a). 
As shown in Figure $2 \mathrm{~b}$, h-surfactant/ $\mathrm{D}_{2} \mathrm{O} / \mathrm{d}_{12}$-cyclohexane is used to isolate scattering from the surfactants, while h-surfactant/8:2 $\mathrm{H}_{2} \mathrm{O}: \mathrm{D}_{2} \mathrm{O} / \mathrm{h}_{12}$-cyclohexane is used to remove contrast from the surfactants, thereby leaving scattering only from the oil-water interface. For $\varphi_{w}=0.01$, differences between the surfactant contrast and interface contrast conditions are indistinguishable for all $q$-values, confirming the formation of microemulsion droplets. However, for $\varphi_{w}=0.04$ and 0.075 (Figure 2a), we observe that the shoulder in the scattering at $q \approx 0.9 \mathrm{~nm}^{-1}$ is apparent only for the surfactant contrast case, and is strongly suppressed for the interface contrast case. This confirms that this shoulder is due to the correlated distance between two adjacent surfactant layers.

To further discern the presence of water between the surfactant layers, we fit the SANS curve for $\varphi_{w}=0.02$ to a core and 3-shell model. The SANS data can be fully fit using scattering length densities corresponding to a cyclohexane core surrounded by 2 surfactant shells with a thin water shell between (Figure 1a). This indicates the formation of swollen vesicle-type oil-in-water-in-oil (O/W/O) double nanoemulsions with a water film thickness of $\approx 6.5 \mathrm{~nm}$. Cryo-TEM corroborates the proposed structure, as we observe uniform nanodroplets with a lighter cyclohexane core and a darker shell composed of water and surfactants (Figure 1b). For $\varphi_{w}=0.04$, in addition to the high- $q$ shoulder at $0.9 \mathrm{~nm}^{-1}$ indicative of correlations between surfactant bilayers, we observe an additional peak at $q \approx 0.3 \mathrm{~nm}^{-1}$, which we believe arises from correlations of structures inside the nanodroplets (Figure 1a). Cryo-TEM for $\varphi_{w}=0.05$ indeed shows multi-core structures, with swollen vesicles having several nanodroplets encapsulated inside. The scattering peak therefore corresponds to the average center-to-center distance between the multiple cores, which we find decreases with increasing $\varphi_{w}$ [5], indicating an increase in the number and therefore crowding of the cores as the water content is increased.

Eventually, as the water content is increased further toward $\varphi_{w}=0.10$, both of the mid- $q$ to high- $q$ shoulders begin to subside, and the scattering curve begins to resemble that where $\varphi_{w}=0.01$. Again, we observe that the curve fits well to a coreshell model, which indicates the formation of W/O nanoemulsions with a water core and surfactant shell. Cryo-TEM confirms the formation of uniform nanodroplets consisting of a lighter water core and a darker surfactant shell.

In summary, using a combination of surfactants with opposite preferred curvature and high-energy emulsification tunes the interfacial curvature to facilitate the production of a series of complex nanoemulsions using high-energy emulsification [5]. A combination of SANS and cryo-TEM were utilized to determine and quantify several complex morphologies including micelles, microemulsions, oil-in-water-in-oil vesicle-type nanoemulsions, multi-core vesicle nanoemulsions, and uniform nanodroplets. Interestingly, the SANS data can be fit to a single morphology

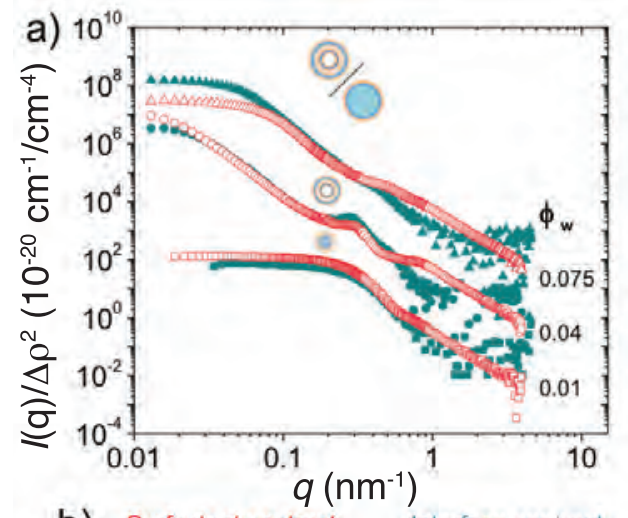

b)
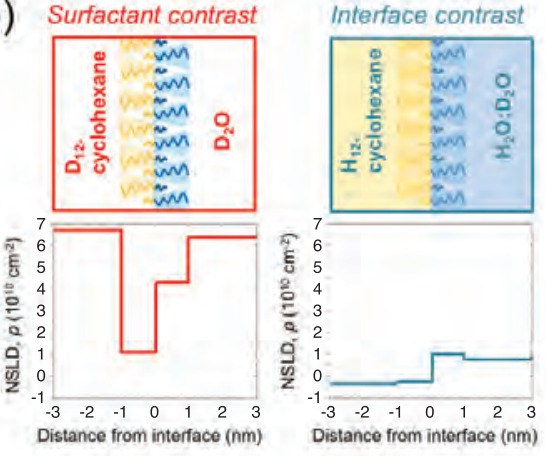

FIGURE 2: a) SANS spectra normalized by the contrast factor, $\Delta \rho^{2}$, for two different surfactant contrasts (red) using h-surfactant $/ \mathrm{D}_{2} \mathrm{O} / \mathrm{d}_{12}$-cyclohexane, and interface contrast (green) using $\mathrm{H}$-surfactant/8:2 $\mathrm{H}_{2} \mathrm{O}: \mathrm{D}_{2} \mathrm{O} / \mathrm{H}_{12}$-cyclohexane - at three water concentrations ( $\left.\phi_{W}=0.01,0.04,0.075\right)$, b) schematic comparing the scattering length density $(\rho)$ distribution across a cyclohexane-surfactant-water interface for each contrast studied.

for each composition, demonstrating that the morphology can be selected by the choice of water content alone, despite the highly non-equilibrium nature of the nanodroplets. Contrast variation helped to confirm the precise location of the different components including the surfactant and water within the complex nanoemulsions. Ongoing efforts are aimed at using these complex structures as templates for producing complex multi-phase nanoparticles.

\section{References}

[1] M. Windbergs, Y. Zhao, J. Heyman, D. A. Weitz, J. Amer. Chem. Soc. 135, 7933 (2013).

[2] A. Matalanis, O. G. Jones, D. J. McClements, Food Hydrocolloids 25, 1865 (2011).

[3] M. E. Helgeson, Curr. Op. Coll. Int. Sci. 25, 39 (2016).

[4] M. Hashimoto, P. Garstecki, H. Stone, G. Whitesides, Soft Matter, 4(7), 1403 (2008).

[5] P. M. de Molina, M. Zhang, A. V. Bayles, M. E. Helgeson, Nano Letters 16(12), 7325 (2016). 


\title{
Small nanoparticles disentangle polymer chains in nanocomposites
}

\author{
E. Senses, ${ }^{1,2}$ S. M. Ansar, ${ }^{3}$ C. L. Kitchens, ${ }^{3}$ Y. Mao, ${ }^{1,2}$ S. Narayanan, ${ }^{4}$ B. Natarajan, ${ }^{5}$ and A. Faraone ${ }^{1}$
}

A ddition of nanoparticles to soft polymeric matrices has long been known to cause unexpected changes in physical properties of the resulting 'polymer-nanoparticle composites' (PNCs) [1]. Despite its relevance to advanced materials applications of PNCs in many areas such as aerospace, biomaterials and flexible electronics, the complex role of nanoparticles on large scale dynamics of polymers remains poorly understood. An intriguing example is the viscosity reduction often reported in composites with nanoparticles smaller than the size of a single polymer chain (typically $5 \mathrm{~nm}$ to $10 \mathrm{~nm}$ ). Such behavior is surprising and has drawn much attention since the classical models, such as Einstein-Batchelor, which predicts always increasing viscosity due to particle addition. While computer simulations predict very rich dynamical effects of nanoparticle size [2], how the microscopic polymer dynamics are affected by the nanoparticles that are as small as the entanglement spacing has not been clarified experimentally mainly due to the lack of spatial and temporal resolution in conventional experimental techniques. Neutron scattering affords such a possibility.

Contrary to rigid nanoparticles, polymers possess structural hierarchy and distinct relaxation processes which in turn control the bulk properties at the monomer and chain level. At short time scales, the monomers exhibit unrestricted motion due to thermal fluctuations (called Rouse motion). At larges scales, the motions are restricted by entanglements due to interpenetration of the chains. Such constrained curvilinear motion of chains was called 'reptation' by Pierre-Gilles de Gennes [3] in analogy to the motion of slithering snakes. The tube model proposed by Edward and Doi [4] assumes that the chains are trapped within a virtual tube formed by entanglements that allows only back and forth motions along the tube contour. It is this motion which gives polymers rubber-like elasticity. From the macroscopic point of view, the flow of the long-entangled linear chains in molten state is then simply determined by (i) the microscopic time-scale at which the monomers relax, i.e., elementary Rouse rate, $\mathrm{W}$, and (ii) the length-scale of the confining tube, i.e., reptation tube diameter, $d$. In this work [5], we directly measured these microscopic properties of polymers in the presence of nanoparticles of different sizes to shed-light into the current discussion on the role of particle size.
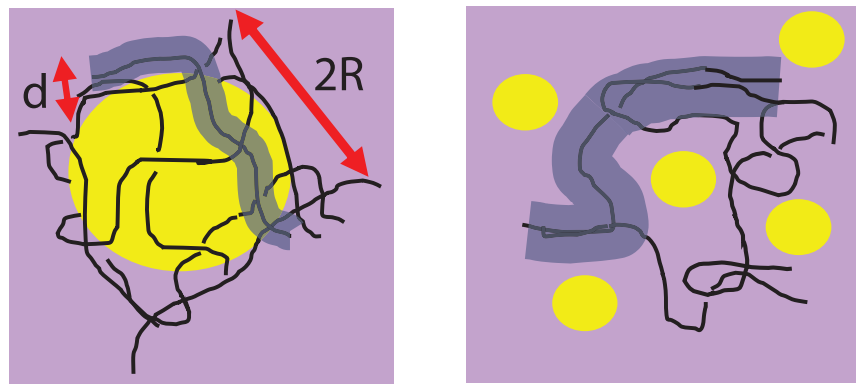

FIGURE 1: Schematic representation of the nanoparticle-polymer composites with particles of size $(2 R)$ larger than (on the left) and smaller than (on the right) the reptation size, $d$. The shaded volume along the contour of the chains illustrates the virtual tube in which the chains reptate.

We prepared nanocomposites made of short (unentangling) poly (ethylene glycol) (PEG) attached to gold (Au) nanoparticles dispersed in long (entangling) poly (ethylene oxide) (PEO) matrix. PEG and PEO are chemically identical, therefore, there is no energetic interactions between the particles and the matrix polymer (i.e., athermal system). We used large $(20 \mathrm{~nm})$ and small $(3.5 \mathrm{~nm})$ nanoparticles at a fraction of $20 \%$ by volume to create cases where the particles are smaller and larger than the reptation tube diameter of the matrix PEO $(\approx 5 \mathrm{~nm}$ ) (see schematic in Figure 1).

The single PEO chains are made visible to neutrons by contrast matching the Au nanoparticles to the PEO matrix. This was achieved by using a mixture of hydrogenated PEO (24 \%) and deuterated PEO (76 \%) of the same length. Figure 2 shows the small-angle neutron scattering (SANS) intensity profiles as a function of wavevector, $Q$. All profiles from particle-free PEO and the nanocomposites converge to give scaling $I(Q) \sim Q^{-2}$ at intermediate length scales; typical of a long polymer chain exhibiting random walk in space and described by Debye function (fitting result shown as line in Figure 2). The rheological properties of the composites (Figure 2 inset) are very different. As expected, the large nanoparticles caused the composite viscosity to increase orders of magnitude. The small nanoparticles, however, decreased the viscosity $\approx 50 \%$ relative to the particle-free matrix, allowing the nanocomposite flow easier.

\footnotetext{
1 NIST Center for Neutron Research, National Institute of Standards and Technology Gaithersburg, MD 20899

2 University of Maryland, College Park, MD 20742

${ }^{3}$ Clemson University, Clemson, SC 29634

${ }^{4}$ Advanced Photon Source, Argonne National Laboratory, Argonne, IL 60439

${ }^{5}$ Materials Measurement Laboratory, National Institute of Standards and Technology Gaithersburg, MD 20899
} 


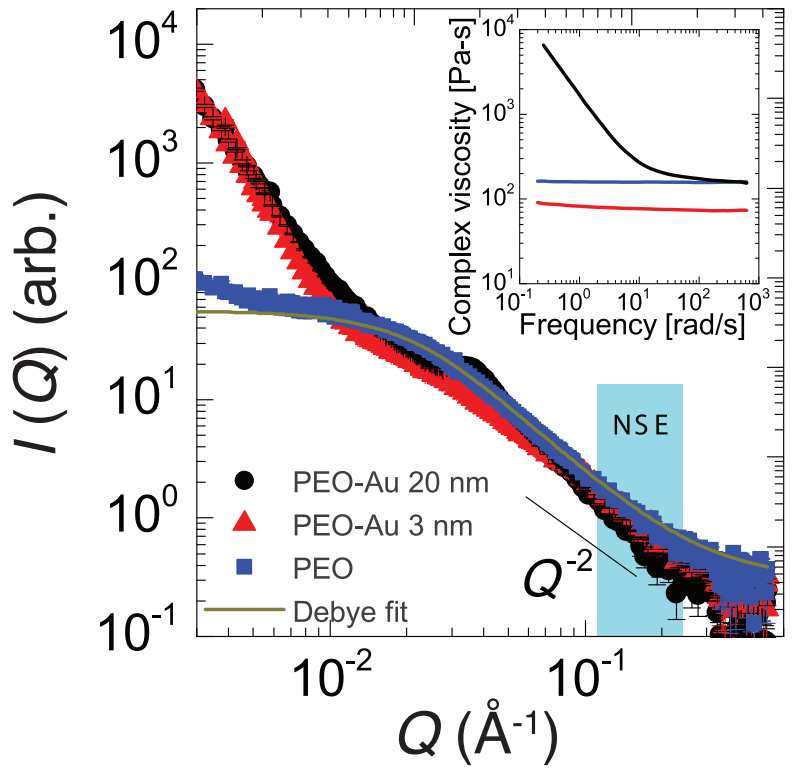

FIGURE 2: Small-angle neutron scattering (SANS) profiles of neat PEO and PEO-Au nanocomposites with $20 \mathrm{~nm}$ and $3 \mathrm{~nm}$ diameter particles zero-average contrast matched to the matrix. The line is the fit to the Debye function (with $I \infty Q^{-2}$ ) typical of a Gaussian polymer chain. The shaded region shows the $Q$-range used in neutron spin-echo measurements [5].

We measured collective PEO dynamics using neutron spin-echo (NSE) technique up to $100 \mathrm{~ns}$. The single chain dynamic structure factor, $S(Q, t)$, is obtained in the spatio-temporal range relevant to a local reptation motion (shaded region in Figure 2). The initial decays in Figure 3a are identical; so, there is no significant effect of particle size on the timescale of the monomeric relaxation. The curves in Figure $3 \mathrm{a}$ are the trends predicted from the Rouse model [6]. Above $10 \mathrm{~ns}$, the motions are restricted by the entanglements. This collective chain dynamics within the confining tube have been formulated by de Gennes' [3] and Doi and Edwards [4] as:

$$
S(Q, t)=\left[1-\exp \left(-\frac{Q^{2} d^{2}}{36}\right)\right] S_{\text {local }}(Q, t)+\exp \left(-\frac{Q^{2} d^{2}}{36}\right) S_{\text {esc }}(Q, t),
$$

where $S_{\text {local }}(Q, t)=\exp \left(\frac{t}{\tau_{0}}\right) \operatorname{erfc}\left(\sqrt{t / \tau_{0}}\right)$ is the local reptation within the tube with characteristic time-scale $\tau_{0} \cdot S_{\text {esc }}(Q, t)$ is the long-time creeping of the chain out of its original tube and $S_{e s c}(Q, t)=1$ for the motions probed by NSE in this work as $t_{N S E} \ll \tau_{R}(\approx 1 \mu \mathrm{s})$. The long-time plateau level is determined by $\exp \left(-\frac{Q^{2} d^{2}}{36}\right)$. Since the monomeric relaxation did not change, the only free parameter, the reptation tube diameter, $d$, was obtained from global-fitting to relaxation at all $Q$-values. Note that the reptation model does not account for the initial unrestricted Rouse motion at short times, fitting was applied for $t>50 \mathrm{~ns}$ (Figure 3b and 3c). The reptation tube size in nanocomposites with large NPs $\left(d_{P E O-20 n m A u}=5.17 \mathrm{~nm}\right.$ $\pm 0.19 \mathrm{~nm}$ ) (Figure $3 \mathrm{~b}$ ) is identical- within the experimental uncertainties- to that for the particle free matrix $\left(d_{P E O}=5.03 \mathrm{~nm}\right.$ $\pm 0.10 \mathrm{~nm}$ ). This contrasts with the increase of the nanocomposite tube diameter with small NPs $\left(d_{P E O-3 n m A u}=6.11 \mathrm{~nm} \pm 0.13 \mathrm{~nm}\right)$, revealed by the lowered long-time plateau level in the nanocomposite with respect to the neat PEO (Figure 3c).
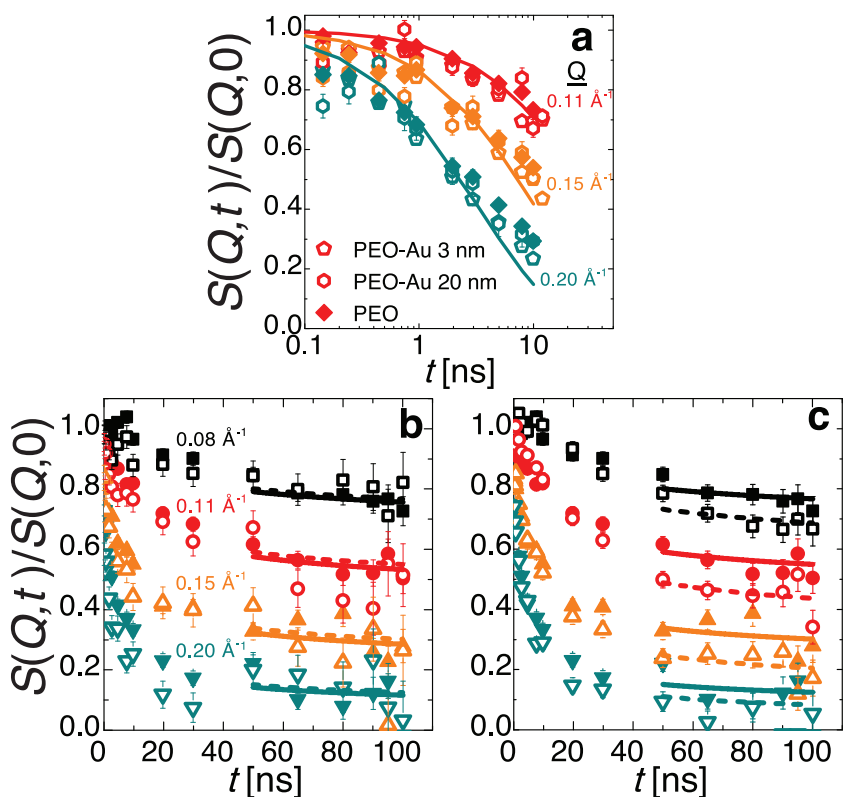

FIGURE 3: Dynamic structure factor of the neat PEO (filled symbols) and the PE0 nanocomposite with $3.5 \mathrm{~nm}$ Au nanoparticles (open symbols) at $T=400 \mathrm{~K}$ showing (a) identical initial Rouse decays at short times and (b) identical longtime plateau for large NP composite and (c) reptation tube dilation of small NP composite. The colors represent different $Q$ values.

These results allow us to reach several important conclusions: (i) using dynamic neutron scattering on isotopically labeled polymers, we showed the first direct experimental evidence of tube dilation in polymer nanocomposites in the small particle limit. (ii) The increase of the reptation tube size by small nanoparticles caused a decrease in entanglement density in the composites without significantly changing the local motion of the monomers. No such effect is seen for nanocomposites with large nanoparticles. (iii) The decreasing entanglements with particles smaller than the entanglement strands enhances the flow of polymer chains in the nanocomposites and can be the reason for the observed viscosity reductions in non-attractive polymer nanocomposites.

\section{References}

[1] R. Krishnamoorti, R. A. Vaia, Polymer nanocomposites: synthesis, characterization, and modeling (ACS Washington, DC, 2002), Vol. 804.

[2] J. T. Kalathi, S. K. Kumar, M. Rubinstein, G. S. Grest, Soft Matter 11, 4123 (2015).

[3] P.-G. de Gennes, J. Chem. Phys. 55, 572 (1971).

[4] M. Doi, S. F. Edwards, The theory of polymer dynamics (oxford university press, 1988), Vol. 73.

[5] E. Senses, S. M. Ansar, C. L. Kitchens, Y. Mao, S. Narayanan, B. Natarajan, A. Faraone, PRL 118, 147801 (2017).

[6] D. Richter, M. Monkenbusch, A. Arbe, J. Colmenero, Neutron Spin Echo in Polymer Systems, 1 (2005). 


\title{
Measuring the interaction of solvated polymers and surfaces
}

\author{
R. J. Sheridan, ${ }^{1}$ S. V. Orski, ${ }^{1}$ R. L. Jones, ${ }^{1}$ S. K. Satija, ${ }^{2}$ and K. L. Beers ${ }^{1}$
}

$\mathrm{T}$ he architecture of advanced custom multiblock polymers lend crucial functionality to new materials for energy storage, drug delivery, and lithography. This functionality is imparted by the combination of the choice of monomer segments and structure and organization of those segments into blocks and branches. Often the quality or efficiency of the desired functionality is dependent on the purity and dispersity of the substituent blocks and branches. Therefore, improvements in the measurement and purification of these polymers leads directly to improvements to functionality in important fields of modern materials science. Among the techniques used to separate and characterize these polymers is Liquid Chromatography at Critical Conditions (LC-CC or just (C). By adjusting one "surface interaction" parameter, one can calculate chromatographic peak elution volumes that coincide with those observed by experiment. We attempt to measure this interaction energy as directly as possible.

In our recent experiment, [1] we produced an end-tethered polystyrene film (PSN) with a sufficiently low grafting density to reveal the surface interactions when probed with a combination of neutron scattering and neutron reflectivity. This grafting strategy solves the challenges posed by the direct measurement of free chains, without totally masking the conformational changes due to surface interaction. (See Figure 1.) Analysis of neutron reflectivity data showed that in the case of moderate solvent quality and attractive surface interaction (cyclohexane- $d_{12}$ ), the surface interaction parameter can be measured with significant precision.

Neutron reflectivity profiles are usually interpreted through the lens of simple slab models, often with roughness approximations for each interface. Instead, we fit our reflectivity curves using the thermodynamic parameters of the Scheutjens-Fleer numerical self-consistent field theory (SF-nSCFT)[2]. The necessary limited grafting density was achieved using a high-temperature "grafting-to" approach, where primary-amine terminated polystyrene was spin-coated over a silicon wafer bearing a sub-monolayer of epoxide-functionalized silanes and then heated to ensure covalent grafting.

In order to build a complete layer model of a swollen end-tethered polymer film, it is necessary to first specify the material parameters of the silicon substrate. The parameters feed forward into our analysis of the swollen PSN films, as well as giving a measurement

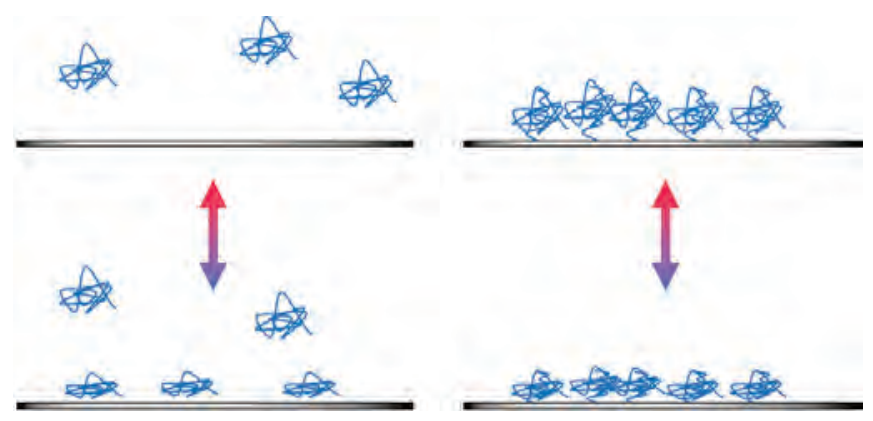

FIGURE 1: Diagram of the response to changing surface interaction in free polymer and our model end-tethered polymer. At high temperatures above the temperature of critical conditions $\left(T_{C C}\right)$ individual chain segments are repelled from the substrate. Below $T_{\text {CC }}$ segments are attracted and a film forms. However, there is not a sufficient mass excess near $T_{\mathrm{CC}}$ to measure the conformation of the adsorbed chains. The end-tethered polymer films are always measurable over the same range of conditions.

of the dry thickness of the PSN film at $34.06 \AA \pm 0.74 \AA$. Following the measurement of substrate parameters, the silicon wafer bearing the PSN end-tethered film was enclosed in a temperature-controlled, sealed environmental cell which was then filled with fresh cyclohexane- $d_{12}$. This cell places the back face of the wafer in contact with a computer-controlled recirculating bath, allowing us to automatically collect backside reflectivity profiles of the swollen PSN film at a range of temperatures, from $10.7^{\circ} \mathrm{C}$ to $52.0^{\circ} \mathrm{C}$. Representative data and corresponding model fits are presented in Figure 2 and Figure 3.

In Figure 2, subtle but clear differences in the behavior of the films are apparent. We interpret these reflectivity shifts through the SF-nSCFT and reflectivity models as the collapsing and swelling of the PSN chains as the solvent quality of cyclohexane- $d_{12}$ changes with temperature. At low temperature, the polymer segments are largely collapsed near the surface with relatively few tails reaching a short distance away from the substrate. As the temperature increases polymer segments swell away from the surface and the tails are able to reach farther into the solution.

Figure 4 demonstrates that by following the protocol laid out in our work, one can obtain a measurement of the surface interaction parameter $\chi_{\mathrm{s}}$ for a given polymer-solvent-surface 


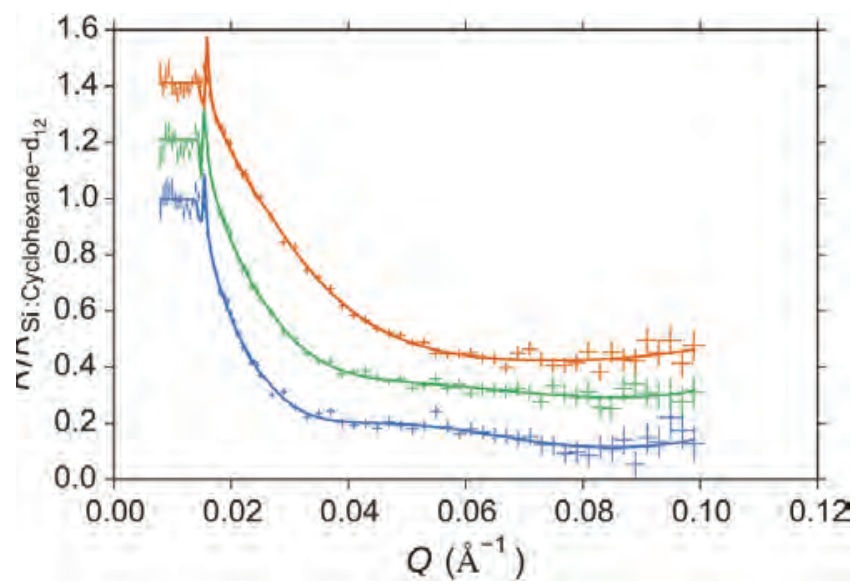

FIGURE 2: Reflectivity data (crosses) and best fit models (lines) of the PSN end-tethered film swollen in cyclohexane- $\mathrm{d}_{12}$ at $10.7^{\circ} \mathrm{C}$ (red), $34.2^{\circ} \mathrm{C}$ (green), and $52.0^{\circ} \mathrm{C}$ (blue). The size of the crosses represents one standard deviation of uncertainty in the reflectivity signal according to Poisson counting statistics (vertical) and wavelength smearing (horizontal).

system. The interaction parameters $\chi$ and $\chi_{\mathrm{s}}$ are plotted in Figure 4 as a function of temperature. $\chi$ decreases with increasing temperature, meaning solvent quality or strength increases, passing through the theta point $\chi=0.5$ at $37^{\circ} \mathrm{C}$ to $40^{\circ} \mathrm{C}$. $\chi_{\mathrm{s}}$ decreases with increasing temperature, meaning the strength of interaction of the polymer with the wall decreases, although the magnitude of the $\chi_{\mathrm{s}}$ parameter is greater than 0.18 (a theoretical adsorption transition point in the SF-nSCFT) across the entire measured temperature range. This means that we observe strongly attractive behavior even at our highest experimental temperature. These observations of $\chi$ and $\chi_{\mathrm{s}}$ are in strong agreement with empirical expectations $[3,4]$.

In our recent publication, [1] a method was presented to measure the thermodynamic surface interaction parameter $\chi_{\mathrm{s}^{\prime}}$ as well as the Flory-Huggins solvent interaction parameter $\chi$, in one experiment using a carefully prepared end-tethered polymer film. Neutron reflectivity data of the film swollen in solvent was collected and a layer model was directly fit with parameters of a self-consistent field theory, allowing for the generation of parameter estimates and uncertainty derived directly from the instrument measurement uncertainties. The temperature dependence of $\chi$ and $\chi_{\mathrm{s}}$ in cyclohexane- $\mathrm{d}_{12}$ was observed and demonstrated agreement with previously reported work.

\section{References}

[1] R. J. Sheridan, S. V. Orski, R. L. Jones, S. K. Satija, K. L. Beers, Macromolecues 50, 6668 (2017).

[2] G. J. Fleer, M. A. Cohen Stuart, J. M. H. M. Scheutjens, T. Cosgrove, B. Vincent, Polymers at Interfaces (Chapman \& Hall, London, 1993).

[3] J. P. Cotton, J. Chem. Phys. 65, 1101 (1976).

[4] P. D. Gallagher, S. K. Satija, A. Karim, J. F. Douglas, L. J. Fetters, J. Polym. Sci. Part B Polym. Phys. 42 (22), 4126 (2004).

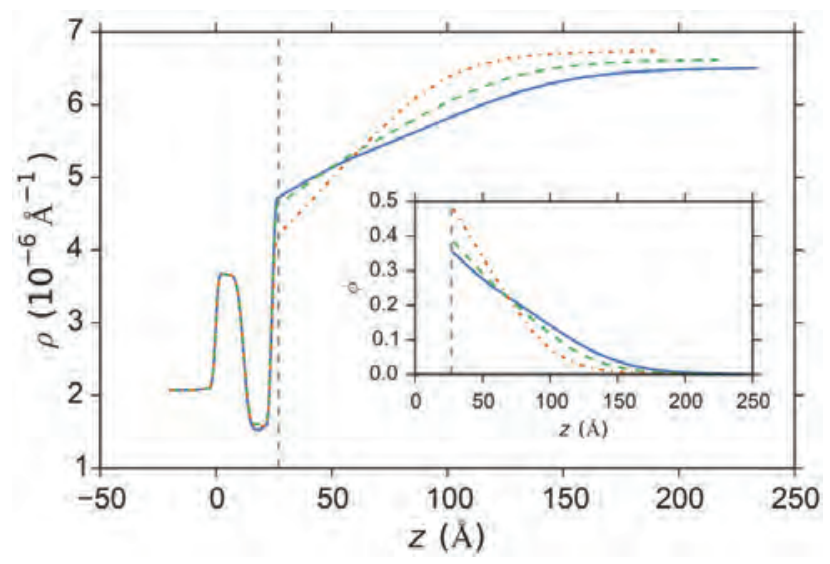

FIGURE 3: Scattering length density curves of PSN end-tethered films swollen in cyclohexane- $\mathrm{d}_{12}$ at $10.7{ }^{\circ} \mathrm{C}$ (red dashed), $34.2^{\circ} \mathrm{C}$ (green dashed), and $52.0^{\circ} \mathrm{C}$ (blue) corresponding to the best fit models of Figure 2. Inset is the corresponding inferred volume fraction of polystyrene.

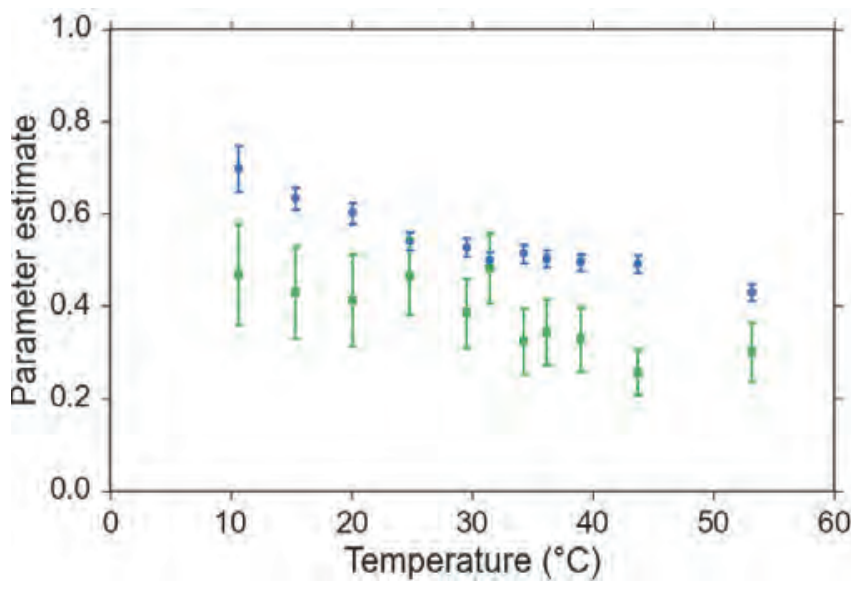

FIGURE 4: $\chi$ (blue circles) and $\chi_{\mathrm{s}}$ (green squares) of polystyrene in cyclohexane- $\mathrm{d}_{12}$ via the SF-nSCFT model as a function of temperature. $\chi$ decreases with temperature indicating increasing solvent quality. $\chi_{\mathrm{S}}$ decreases with temperature indicating decreasing polymer-surface interaction. 


\title{
Understanding the recovery of tough, nanostructured hydrogels after deformation
}

\author{
C. G. Wiener, ${ }^{1}$ C. Wang,${ }^{1}$ Y. Liu, ${ }^{2}$ R. A. Weiss, ${ }^{1}$ and B. D. Vogt ${ }^{1}$
}

1 ydrogels are soft materials with solid-like mechanical properties that contain a significant fraction of water, in some cases exceeding water mass fractions of 0.99 The ability to maintain solid shape despite the significant water content is driven by two factors: the formation of a threedimensional network that encompasses the hydrogel and the large water affinity of the network. In many cases, hydrogels consist of a crosslinked hydrophilic polymer, where without the crosslinks the polymer would dissolve in water. The formation of a network from the crosslinking leads to a polymer that consists effectively of a single molecule that spans the macroscopic dimensions of a hydrogel. The hydrophilicity of the polymer and the crosslink density control the water content of a hydrogel through the modulation of the osmotic pressure and the stretching of the polymer segments between crosslinks. The equilibrium water content is thus determined by the interplay between these enthalpic and entropic driving forces. As the water content increases, the hydrogel tends to become softer. The combination of the ability to hold significant water content and solid-like properties provides opportunities for a wide variety of applications encountered in many consumer products including cosmetics, agriculture, pharmaceuticals, sealants, food additives, tissue engineering, and wound dressings. For example, the efficacy of diapers to prevent leaks is driven by the crosslinked superabsorbent polymer that can absorb water with nearly 300 times its weight, which is effectively a hydrogel. Moisture control potting soil also contains a simple hydrogel. Similarly, hydrogels are added to shampoo to help lock in moisture and nutrients.

The large water content and soft nature of hydrogels can appear to be a good mimic of natural tissue as the human body is composed of nearly $70 \%$ water by weight. However, most synthetic hydrogels exhibit an Achilles' heel when it comes to their mechanical properties as they are brittle. Anyone who has torn their contact lenses, which are hydrogels, can attest to the small force necessary for a crack to propagate. The origins of this weakness come from the highly stretched initial state of the polymer segments between crosslinks due to their large swelling from the absorbed water. When the hydrogel is stretched, the polymer segments become fully elongated and the force is sufficient to break covalent bonds in the network, which increases the load required for neighboring network strands to hold. For many emergent applications, increased toughness in hydrogels is also critical. The toughness of hydrogels can be significantly

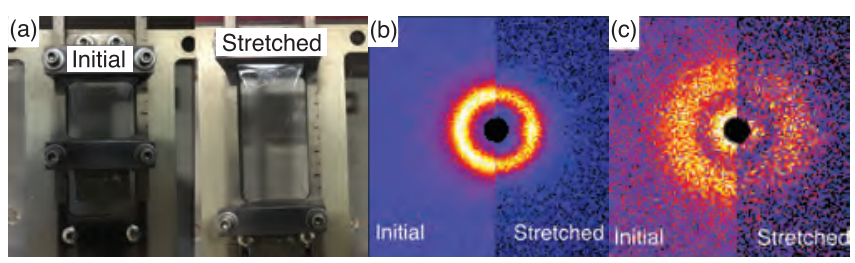

FIGURE 1: (a) Picture of hydrogel before and after stretching to $150 \%$ strain. Contrast match SANS is used to elucidate the (b) structure factor between crosslinks and (c) form factor of the crosslinks.

enhanced by the introduction of an energy dissipation mechanism that releases energy before the rupture of covalent bonds in the network [1, 2]. By using non-covalent interactions such as hydrogen bonding or hydrophobic associations as crosslinks, energy can be dissipated by the dissociation of these crosslinks, but then the crosslinks can reform to re-generate the network without the loss of mechanical properties [2]. This toughening mechanism relies on the reformation of the associations to maintain the properties of the hydrogel. As such, the kinetics of how these associations reform after stretching is important to the design of tough hydrogels.

This report considers the stress relaxation of a model hydrophobically modified hydrogel $[2,3]$ that is formed by swelling a random copolymer of 2-(N-ethylperfluorooctane sulfonamide)ethyl acrylate (FOSA) and N,N-dimethylacrylamide (DMA) to equilibrium in deionized water. The DMA is water soluble, but the copolymer is unable to dissolve due to the formation of aggregated domains of the hydrophobic FOSA. These hydrophobic aggregates act as physical crosslinks for the hydrogel that contains $70 \%$ water by weight. These hydrogels are transparent as shown in Figure 1a and can be elongated over $400 \%$ before the failure. Due to the perfluoronation of the hydrophobic component, the neutron scattering length density of the FOSA aggregates is much higher than that of the DMA. This allows the nanostructure to be interrogated readily with small-angle neutron scattering using contrast variation to selectively probe the structure of either DMA or FOSA. Using a mixture of $\mathrm{D}_{2} \mathrm{O} / \mathrm{H}_{2} \mathrm{O}$ with a volume fraction of $27 \% \mathrm{D}_{2} \mathrm{O}$, matches the scattering length density (SLD) of the DMA phase. The resultant scattering is predominately due to the structure factor of the FOSA aggregates associated with their distribution (average spacing). Despite the random nature of the copolymer, the distribution of the FOSA aggregates is regular, which results in a correlation peak in the 2D scattering pattern (Figure 1b). When the hydrogel is elongated by $150 \%$, the scattering becomes

\footnotetext{
1 University of Akron, Akron, $\mathrm{OH} 44313$

2 NIST Center for Neutron Research, National Institute of Standards and Technology, Gaithersburg, MD 20899
} 

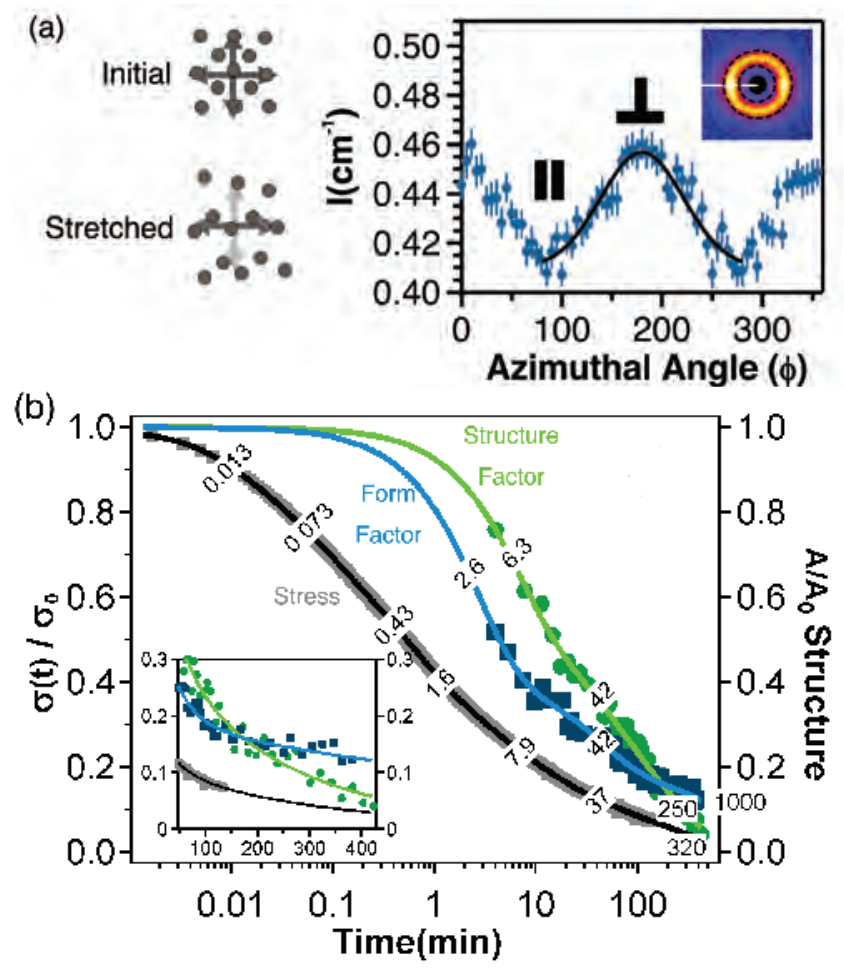

FIGURE 2: (a) Azimuthal dependence of the scattering as measured $\approx 4$ min after the step strain to $150 \%$. (b) Time dependence of the stress and intensity anisotropy following the step strain. The structure factor is shown as green data points and the form factor in blue (right vertical axis). The stress relaxation is in black (left vertical axis). Time values are marked on the data, and the inset is the same data on a linear time scale.

anisotropic with a decrease in scattered intensity in the direction of the deformation, demonstrating the response at the nanoscale to the macroscopic deformation. Similarly, the shape (form factor) of the FOSA aggregates themselves can be determined from scattering using a mixture of $\mathrm{D}_{2} \mathrm{O} / \mathrm{H}_{2} \mathrm{O}$ with a volume fraction of $\mathrm{D}_{2} \mathrm{O}$ of $50 \%$, which matches the FOSA, but non-hydrated DMA segments surround the FOSA aggregates to provide the contrast to elucidate information about the aggregates themselves as shown in Figure 1c.

The anisotropy of the scattering patterns that develops from stretching the hydrogels is used to examine how the nanostructure recovers after a step strain to $150 \%$. This anisotropy is better illustrated by the 1D patterns of the average intensity of the peak $Q=(0.092 \pm 0.024) \AA^{-1}$ for the structure factor (interdomain spacing) and at $Q=(0.14 \pm 0.047) \AA^{-1}$ for the form factor (aggregate size) as shown in Figure 2a. The decay in this anisotropy provides a measure for the recovery of the nanostructure, which should be directly related to the stress relaxation. Figure $2 \mathrm{~b}$ shows the stress relaxation associated with the $150 \%$ step strain. In order to relate the nanostructure relaxations to the stress relaxation, a Generalized Maxwell Model (GMM) with 7 relaxation times was found to describe the data well (solid black line), which results in relaxation times of $\tau_{n}=0.0125 \mathrm{~min}, 0.0730 \mathrm{~min}, 0.432 \mathrm{~min}, 1.55 \mathrm{~min}, 7.94 \mathrm{~min}$, $37.0 \mathrm{~min}$ and $323 \mathrm{~min}$. Similarly, the relaxation in the anisotropy amplitude associated with the FOSA aggregate and the DMA network chains can be fit with an analog of the GMM, but using 3 relaxation times as shown in Figure $2 b$ by the solid lines.

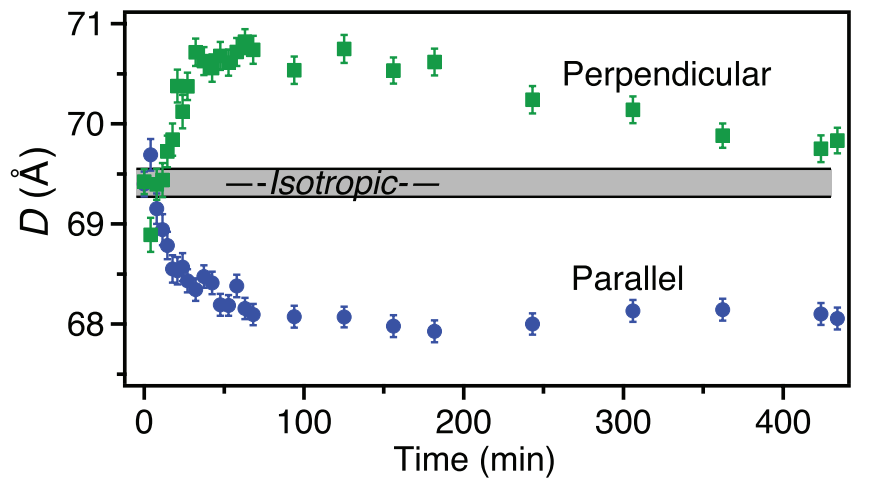

FIGURE 3: Changes in the network nanodimensions are long lived parallel to the stretching direction, while the distance between crosslinks (perpendicular to the deformation) recovers to the initial state (grey area).

Intriguingly, the best fits for the associated relaxation time appear to correlate quite well in $\tau_{n}$ from the stress relaxation except for one longer relaxation time in the FOSA aggregates (950 min). The lack of this longer timescale in the stress relaxation may be due to lack of data beyond $120 \mathrm{~min}$.

The physical dimensions of the nanostructure also become anisotropic as shown in Figure 3 for the interdomain spacing, $D$. During stress relaxation, $D$ in the parallel (perpendicular) direction rapidly decrease (increase). For the parallel direction, $D$ after stress relaxation is smaller than $D$ of the initial hydrogel. As the hydrogel remains macroscopically strained to $150 \%$ during the stress relaxation, it is not unreasonable that the structure does not completely recover to its original state. There is a slow recovery in the perpendicular direction towards the initial unstretched dimension, while the parallel direction remains compressed. This result is attributed to the chain statistics associated with breaking and reforming aggregates, where the more stretched domains are more apt to break apart through a pull-out mechanism, but the reformation of the domains favors smaller dimensions.

These combined measurements provide valuable insight into the mechanisms associated with the energy dissipation in tough hydrogels. Neutron scattering enables the interrogation of separate components of these hydrogels to understand the energy dissipation (chain pull-out) and the recovery process. The relaxation processes on the nanoscale of the DMA network chains and the FOSA aggregates can be directly related to macroscopic stress relaxation of the hydrogel. For combating fatigue in these tough hydrogels, understanding the time scales of the recovering of the different components may provide important information to improve the fatigue resistance in tough hydrogels. The structural insight into dynamic processes in tough hydrogels may also provide new concepts for the design of these important materials.

\section{References}

[1] S. Shams Es-haghi, A. I. Leonov, R. A. Weiss, Macromolecules 47 (14), 4769 (2014).

[2] J. Hao, R. A. Weiss, Macromolecules 44 (23), 9390 (2011).

[3] C. G. Wiener, C. Wang, Y. Liu, R. A. Weiss, B. D. Vogt, Macromolecules 50 (4), 1672 (2017). 


\title{
Fingerprinting molecular deformation of polymers
}

\author{
Y. Wang, ${ }^{1}$ Z. Wang, ${ }^{2}$ C. N. Lam, ${ }^{1}$ W.-R. Chen, ${ }^{2}$ and Y. Liu ${ }^{3,4}$
}

S mall-angle neutron scattering (SANS) is an excellent tool for investigating the conformational changes of polymers under flow and deformation, because of its ability to retrieve information about chain statistics over a wide range of length scales. Recently, a novel approach for analyzing the two-dimensional SANS spectrum of deformed polymers has emerged from our investigation, imparting new power to this microscopic experimental technique [1]. The traditional method of SANS data analysis focuses on the radius of gyration tensor, which offers only a coarse-grained picture of molecular deformation on large length scales. Additionally, the traditional approach is evidently inadequate in dealing with complex scattering patterns such as "butterfly" and "lozenge" shapes. By combining and modestly extending several key conceptual ingredients in the literature, our recent work demonstrates how the fingerprint features of macromolecular deformation can be quantitatively determined by the spherical harmonic expansion technique.

In the context of deformed, isotopically labelled polymer melts, the measured scattering signal is approximately proportional to the single-chain structure factor of the polymer chain $S(\boldsymbol{Q}): S(\boldsymbol{Q}) \approx I(\boldsymbol{Q}) /\left[\lim _{Q \rightarrow 0} I_{\text {iso }}(Q)\right]$, where $I_{\text {iso }}(Q)$ is the scattering intensity from the isotropic sample. Formally, the dependence of $S(\boldsymbol{Q})$ on the magnitude $(Q)$ and orientation $(\boldsymbol{\Omega})$ can be expressed in terms of spherical harmonics:

$S(\boldsymbol{Q})=\sum_{l, \mathrm{~m}} S_{l}^{\mathrm{m}}(Q) Y_{l}^{\mathrm{m}}(\mathbf{\Omega})$,

where $S_{l}^{\mathrm{m}}(Q)$ is the expansion coefficient corresponding to the real spherical harmonic function $Y_{l}^{\mathrm{m}}(\boldsymbol{\Omega})$. This approach allows us to decompose the measured $2 \mathrm{D}$ scattering spectrum into components with different symmetry. Because of the axial symmetry of the uniaxial extension problem, all the $m \neq 0$ terms and the odd $l$ terms are forbidden. It follows that

$S(\boldsymbol{Q})=\sum_{l: \mathrm{even}} S_{l}^{0}(Q) Y_{l}^{0}(\theta, \phi)$,

with $Y_{l}^{0}(\theta, \phi)=\Theta_{l}(\theta)=\sqrt{2 l+1} P_{l}(\cos \theta)$ and

$S_{l}^{0}(Q)=\frac{1}{2} \int_{0}^{\pi} \sin \theta \mathrm{d} \theta I_{x z}(Q, \theta) \Theta_{l}(\theta) /\left(\lim _{Q \rightarrow 0} l_{\text {iso }}(Q)\right.$. Physically, each coefficient $S_{l}^{0}(Q)$ represents the $Q$-dependent deformation anisotropy corresponding to the spherical harmonic (Legendre) function $Y_{l}^{0}(\theta, \phi) . S_{0}^{0}(Q)$ is the isotropic component of the SANS (a)

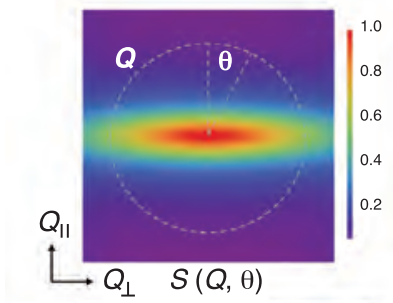

(b)

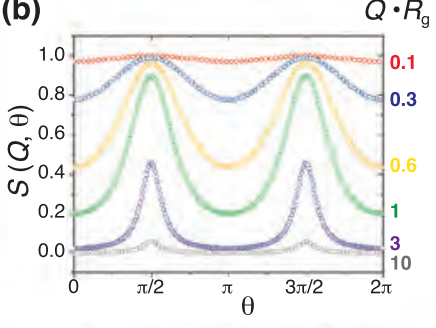

(c) 1.0

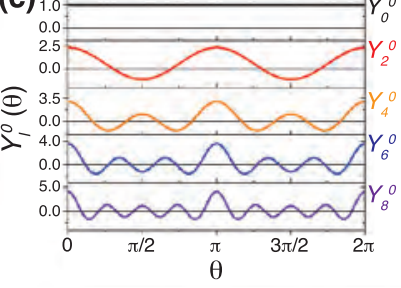

(d)

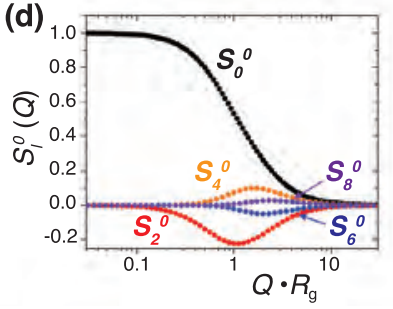

FIGURE 1: Illustration of the spherical harmonic expansion approach with a simulated spectrum. (a) 2D SANS spectrum from the affine model for a linear polymer uniaxially stretched to $\lambda=3$. (b) Angular dependence of the anisotropic single-chain structure factor at various $Q$ 's. (c) Angular dependence of the projections of the spherical harmonic functions on the $x z$-plane. As discussed in the text, these are essentially Legendre functions. (d) The $Q$-dependent expansion coefficients $S_{l}^{0}(Q)$ are given by Legendre expansion of $S(Q, \theta)$.

spectrum, whereas $S_{2}^{0}(Q)$ is the leading term of the anisotropic components.

Our proposal is to systematically employ the spherical harmonic expansion technique in the SANS studies of deformed polymers, extracting and quantitatively analyzing the wave-number dependent expansion coefficients. To further illustrate this idea, let us consider the single-chain structure factor a uniaxially stretched polymer, simulated by using the affine model (Fig. 1). Expanding the angular dependence of the structure factor at different $Q^{\prime}$ s in terms of spherical harmonics, we can translate the 2D spectrum into a 1D plot of $Q$-dependent coefficients $S_{l}^{0}(Q)$. Such an approach provides a new means to distill molecular deformation from anisotropic SANS spectrum. While spherical harmonic analysis has been extensively used in the computer simulations of non-Newtonian simple liquids, notably by Evans and coworkers [2], as well as SANS investigations of colloidal suspensions [3], its potential in understanding the

\footnotetext{
1 Center for Nanophase Materials Sciences, Oak Ridge National Laboratory, Oak Ridge, TN 37831

2 Biology and Soft Matter Division, Oak Ridge National Laboratory, Oak Ridge, TN 37831

${ }^{3}$ NIST Center for Neutron Research, National Institute of Standards and Technology, Gaithersburg, MD 20899

${ }^{4}$ University of Delaware, Newark, DE 19716
} 
macromolecular flow phenomenon has not yet been fully appreciated by the soft matter community.

In a recent work, we applied this spherical harmonic expansion approach to survey a long-standing problem in polymer physics regarding the molecular relaxation in entangled polymers after a large step deformation [1]. The classical tube theory of Doi and Edwards [4] predicts that immediately after the deformation, the polymer chain must retract within the "tube" - a trap imposed by the topological constraints of neighboring chains, until it recovers its equilibrium contour length. This so-called chain retraction hypothesis is the cornerstone of the tube theory for nonlinear rheology of entangled polymers. Although small-angle neutron scattering has long been considered the ideal tool for critically examining this key ingredient of the tube theory, the SANS investigations in the past several decades, which focus exclusively on the analysis of the radius of gyration $\left(R_{\mathrm{g}}\right)$ tensor, have not led to a clear conclusion.

The spherical harmonic expansion technique allows us to bypass the difficulty associated with the traditional $R_{\mathrm{g}}$ analysis, and resolve the controversy in the literature regarding the chain retraction hypothesis in an unambiguous way. The upper panel of Fig. 2 shows the theoretical predictions by the GLaMM model [5], the state-of-the-art version of the tube model, for the expansion coefficient $S_{2}^{0}(Q)$ of a polymer with 34 entanglements per chain during stress relaxation. The chain retraction mechanism leads to two prominent spectral features: the peak shift of $S_{2}^{0}(Q)$ and the increase of anisotropy in the intermediate $Q$ range (anisotropy inversion). Our smallangle neutron scattering measurement on uniaxially stretched entangled polystyrene melts reveals, however, that these characteristic patterns are not experimentally observed. The peak shift is negligible up to 20 times of the Rouse relaxation time and the anisotropy decays monotonically with time at all $Q^{\prime}$ s. This finding directly challenges the prevailing tube model of polymer motion at large strains. The details of this research can be found in Ref. [1].

In conclusion, the spherical harmonic expansion technique provides a convenient platform to bring together rheo-SANS experiments and computational studies of deformed polymers. It has helped to successfully resolve the controversy surrounding one of the central hypotheses of the tube model. We believe this spectrum decomposition method will find many other
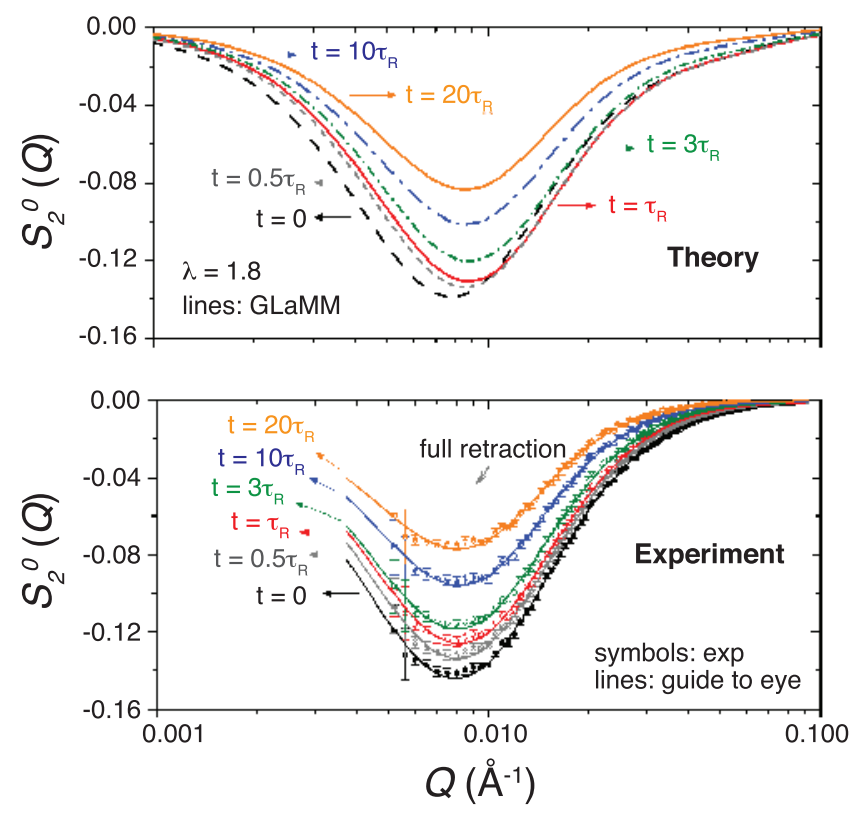

FIGURE 2: (a) Evolution of the spherical harmonic expansion coefficient $S_{2}^{0}(Q)$, as predicted by the GLaMM model [5] for an entangled polystyrene $(Z=34)$ after a step deformation of $\lambda=1.8$, performed with a constant crosshead velocity $v=40 l_{0} / \tau_{\mathrm{R}}$. Here $\tau_{\mathrm{R}}$ is the Rouse relaxation time of the polymer chain and $l_{0}$ is the initial length of the sample. (b) Experimental results on $S_{2}^{0}(Q)$. The theoretically predicted features of chain retraction were not experimentally observed.

useful applications in SANS studies of flow and deformation of macromolecules.

\section{References}

[1] Z. Wang et al., Phys. Rev. X 7, 031003 (2017).

[2 D. J. Evans, H. J. M. Hanley, S. Hess, Phys. Today 37, 26 (1984).

[3] N. A. Clark, B. J. Ackerson, Phys. Rev. Lett. 44, 1005 (1980).

[4] M. Doi and S. F. Edwards, The Theory of Polymer Dynamics (Oxford University Press, Oxford, 1986).

[5] R. G. Graham, A. E. Likhtman, T. C. B. McLeish, S. T. Milner, J. Rheol. 47, 1171 (2003). 


\title{
Peering inside fuel cell electrodes
}

\author{
D. S. Hussey, J. M. LaManna, E. Baltic, D. L. Jacobson
}

$\mathrm{N}$ eutron imaging is the only non-destructive method capable of measuring the water content of hydrogen fuel cells (see Figure 1) during operation, in cell constructions that closely mimic those used for automotive applications. As a result, neutron imaging plays a key role in understanding water transport phenomena in the gas flow channels (with characteristic size of $1 \mathrm{~mm}$ ) and in the gas diffusion layer (characteristic size $250 \mu \mathrm{m}$ ). However water transport in the very heart of the fuel cell, the membrane electrode assembly (MEA) has been challenging up to now. Since the characteristic size of the MEA is about $30 \mu \mathrm{m}$ and the best spatial resolution of neutron imaging detectors is about $15 \mu \mathrm{m}$, neutron imaging could not provide any meaningful insight into the goings-on in automotive MEAs.

That has all changed with a new neutron imaging detector that has at least $2 \mu \mathrm{m}$ spatial resolution and enables the study of the MEA with neutron imaging [1]. The spatial resolution limitation for neutron detectors stems from the range of the charged particles that are emitted as a result of the neutron capture event. For example, in lithium-based detectors, the alpha particle and triton have a combined range of about $50 \mu \mathrm{m}$. Microchannel plate detectors are limited in resolution due to the diameter of the channels of about $6 \mu \mathrm{m}$ resulting in a best resolution of about twice this. The new detector uses a gadolinium oxysulfide (GdOx) scintillator. When Gd captures a neutron, there are several decay chains involving the emission of conversion electrons with energy less than about $150 \mathrm{keV}$ which give off photons as they deposit energy in the scintillator. By magnifying and amplifying the image of the scintillation light, it is possible to see individual neutron capture events in a GdOx scintillator, see Figure 2. It turns out that the scintillation events are somewhat peaked, which allows one to improve the spatial resolution by calculating the center of mass (centroiding) of the scintillation event. The resolution can be improved by about the square root of the number of photons detected. The resolution for $\mathrm{GdOx}$ is between $10 \mu \mathrm{m}$ to $20 \mu \mathrm{m}$, and the detector collects about 100 photons, so one would expect $1 \mu \mathrm{m}$ to $2 \mu \mathrm{m}$ spatial resolution. As Figure 2e shows, the demonstration detector has $2 \mu \mathrm{m}$ resolution. This new imaging capability has been used for studying the water content in fuel cell MEAs with different catalyst layer compositions and to look at the water content at different operating conditions [2].

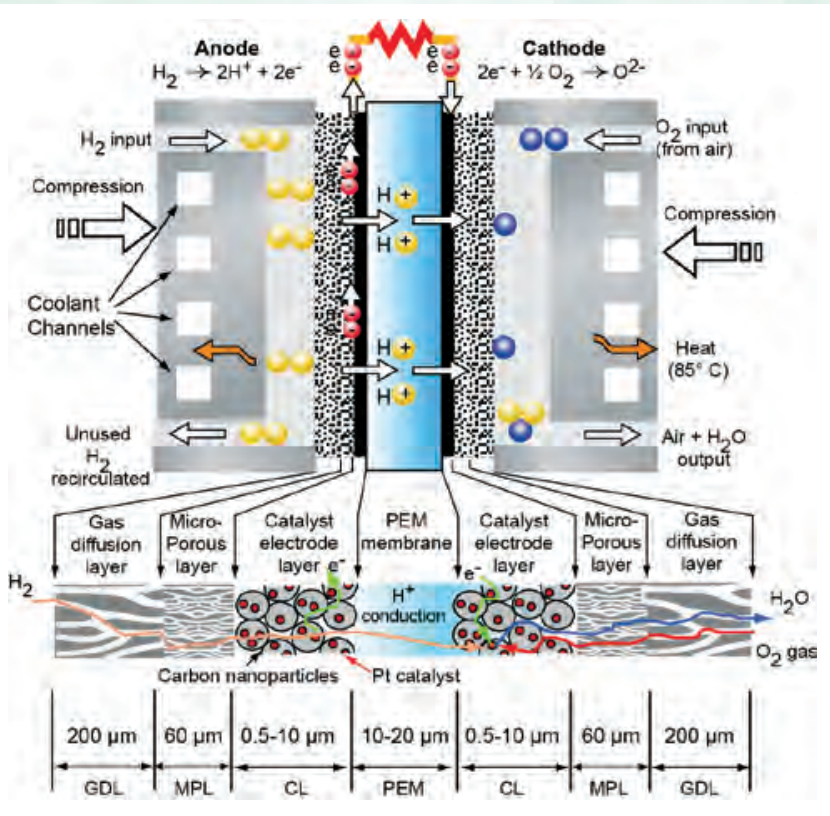

FIGURE 1: Schematic diagram of a hydrogen fuel cell. The MEA is composed of the proton exchange membrane (PEM) and the anode and cathode catalyst layers (CLS).

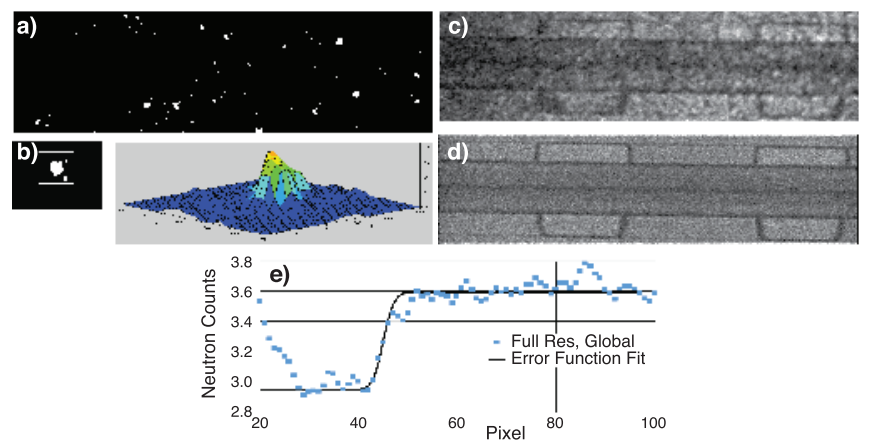

FIGURE 2: An intensified, infinity-corrected microscope viewing a Gd0x screen results in a centroiding detector with $2 \mu \mathrm{m}$ spatial resolution. a) Binarized $3 \mathrm{~ms}$ frame after threshold, b) Zoom-in of a single neutron capture event, c) Sum of 3k frames after threshold, d) Centroided image from 80k frames, e) Error function fit confirming $2 \mu \mathrm{m}$ resolution.

\section{References}

[1] D. S. Hussey, J. M. LaManna, E. Baltic, D. L. Jacobson, Nuclear Instruments and Methods-A, (2017) doi 10.1016/j.nima.2017.05.035.

[2] D. S. Hussey, J. M. LaManna, E. Baltic, D. L. Jacobson, S. Stariha, D. Spernjak, R. Mukundan, R. L. Borup, ECS Transactions of the 232 Meeting of the ECS (2017). 


\title{
Monte Carlo simulation algorithm for B-DNA
}

\author{
S. C. Howell, ${ }^{1}$ X. Qiu, ${ }^{2}$ and J. E. Curtis ${ }^{1}$
}

$\mathrm{U}$ nderstanding the structure-function relationship of biomolecules containing DNA has motivated experiments aimed at determining molecular structure using methods such as small-angle neutron scattering (SANS). SANS experiments provide low-resolution structural information related to characteristic internal distances, as well as the overall size and shape of the scattering particles. When atomic models exist for a system, additional structural information can be extracted from SANS data by matching theoretical scattering profiles from model structures to the experimental scattering data. The SASSIE application [1], developed at the NCNR, includes functionality to rapidly generate ensembles of molecular structures containing regions of flexible amino acid or single-stranded nucleic acid. There are a variety of algorithms available to simulate DNA but they typically cannot sample a large number of viable structures. We have developed a Monte Carlo (MC) algorithm for simulating the native form of double-stranded DNA, also known as B-DNA, to generate an ensemble of plausible structures that can be filtered using experimental results to identify a sub-ensemble of conformations that reproduce the solution scattering of DNA macromolecules [2]. Our algorithm extends the functionality of SASSIE, adding B-DNA to the molecule types it can simulate.

The $M C$ algorithm rapidly generates an ensemble of robust molecular structures through an iterative cycle in which an atomistic B-DNA model is represented using a coarse-grained (CG) bead-rod model, new configurations are generated by sampling bend and twist moves, then atomic detail is recovered by back mapping from the final CG configuration. Using this algorithm on commodity computing hardware, one can rapidly generate an ensemble of atomic level models, each model representing a physically realistic configuration that could be further studied using molecular dynamics.

Figure 1 illustrates the CG bead-rod model used, which represents DNA using $N$ beads connected by $N-1$ inextensible rods of length $l$, for a total contour length, $L=(N-1) l[3]$. This model parameterizes the bending energy using a discretized form of the wormlike chain model [3]:

$$
U_{\text {bend }}=k_{B} T \frac{L_{p}}{l} \sum_{\mathrm{k}=1}^{N-2}\left(1+\boldsymbol{u}_{k} \cdot \boldsymbol{u}_{k+1}\right)
$$

where $k_{B}$ is the Boltzmann constant, $T$ is the temperature, $L_{p}$ is the experimentally determined salt dependent persistence length of B-DNA and $\boldsymbol{u}_{k}$ is the unit vector along the $k^{\text {th }}$ rod. For the

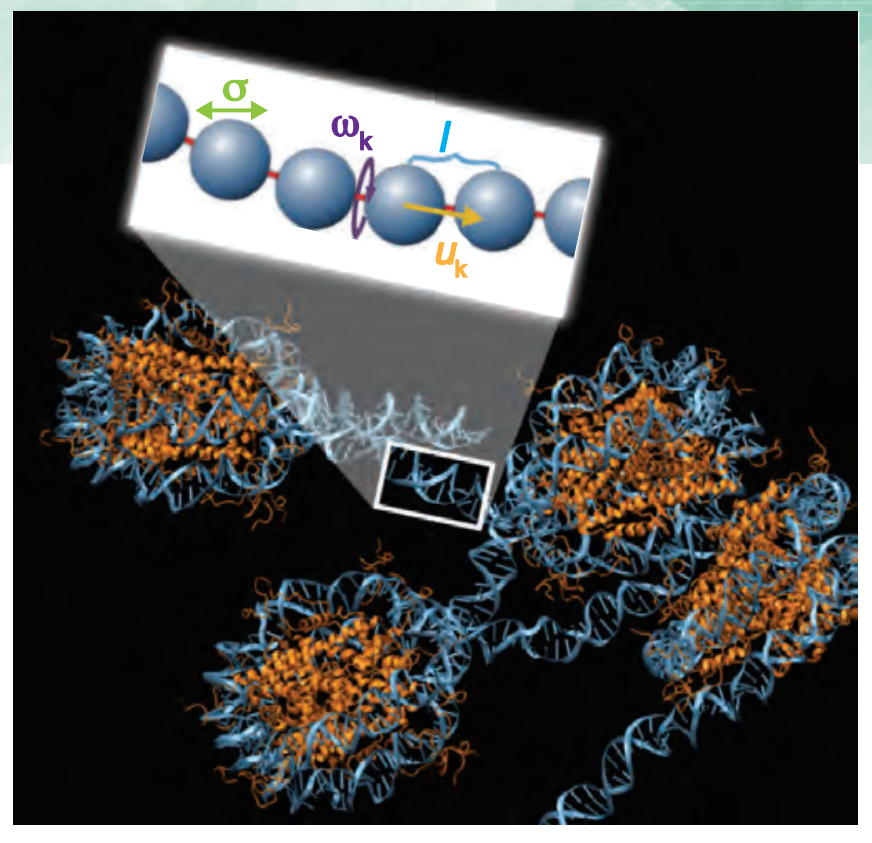

FIGURE 1: Illustration of the bead-rod model and pertinent parameters used for the coarse-grain representation of B-DNA.

twist energy, we represented the bond between adjacent base pairs as a harmonic oscillator of the form:

$$
U_{\text {twist }}=\frac{k_{B} T}{2} \kappa \sum_{\mathrm{k}=1}^{N-1}\left(\omega_{\mathrm{k}}-\bar{\omega}\right)^{2},
$$

where $\kappa=0.062 \times\left(1^{\circ}\right)^{-2}$ is the average twist force constant, $\omega_{\mathrm{k}}$ is the twist angle between base pairs $k$ and $k+1$, and $\bar{\omega}=+35.4^{\circ}$ is the average twist angle [4]. Note that CG moves strain the O3'-P bond between adjacent bases, and the final structures must undergo energy minimization to relax this strain.

This algorithm effectively generates structures spanning a wide range of configuration space in a rapid manner. This algorithm could be beneficially applied to nucleosomes, nucleosome arrays, and other similar systems. Such systems are particularly interesting in light of better understanding the connection between chromatin structure and its connection to gene regulation.

\section{References}

[1] J. E. Curtis, S. Raghunandan, H. Nanda, S. Krueger, Comput. Phys. Commun. 183, 382 (2012).

[2] S. C. Howell, X. Qiu, J. E. Curtis, J. Comput. Chem. 37, 2553 (2016).

[3] J. Wang, H. Gao, J. Chem. Phys. 123, 84906 (2005).

[4] W. K. Olson, A. A. Gorin, X. J. Lu, L. M. Hock, V. B. Zhurkin, Proceed. Nat. Acad. Sci. 95, 11163 (1998).

\footnotetext{
1 NIST Center for Neutron Research, National Institute of Standards and Technology, Gaithersburg, MD 20899

2 The George Washington University, Washington DC, 20052
} 


\title{
Improved sample characterization with simultaneous neutron and X-ray tomography
}

\author{
J. M. LaManna, D. S. Hussey, E. Baltic, and D. L. Jacobson
}

$\mathrm{N}$ eutrons and $\mathrm{X}$-rays provide two nondestructive tomography techniques to probe the three-dimensional structure of samples of interest. Due to the differences in the way neutrons and $\mathrm{X}$-rays interact with atoms, a significant complementarity can be gained from the combination of the two modalities. This complementarity is most evident when investigating hydrogen rich systems such as water or hydrocarbon flow in porous materials found in fuel cells or geological systems, hydrogen rich phases that impact the strength of curing concrete, or lithium rich systems found in many batteries. Neutrons excel at identifying and separating the hydrogen or lithium rich phases from the underlying structure whereas $X$-rays perform best at separating the solid phase from the low atomic mass or fluid phases. Combining the two modalities gives a more complete information set on a sample than what either mode can give on its own. Historically, combining neutron and X-ray images meant that a sample would have to be sent to two separate facilities which could mean several weeks of time between the scans making time sensitive analysis impossible. A system has been developed at NIST to allow for simultaneous neutron and X-ray tomography by orienting a micro-focus X-ray generator perpendicular to the neutron beam. This orientation facilitates the study of slowly dynamic systems such as curing concrete and stochastic processes such as water accumulation in fuel cells.
The system utilizes a $90 \mathrm{keV}$ micro-focus $\mathrm{X}$-ray generator to provide tunable $X$ ray energy and brilliance for tomography. This energy provides reasonable $X$-ray penetration to match neutron penetration on smaller samples allowing for the use of sample environment such as flow cells and high-pressure cells. Two separate detectors are used to image the beams independently allowing spatial and temporal resolution to be tuned for each mode. Typically, the system is operated where the spatial resolution and acquisition time of the $\mathrm{X}$-ray detector is matched to the configuration of the neutron detector. The volumes are aligned after the three-dimensional volume reconstruction to correct for the $90^{\circ}$ offset due to instrument geometry.

Current applications have included the determination of structure of gas bearing shales [1], water distribution in an operating fuel cell, and identification of degradation modes in concrete, among others, with papers discussing system design and initial results due to arrive shortly. The Neutron and X-ray Tomography (NeXT) system allows researchers to capture neutron and X-ray tomography simultaneously [2]. By capturing both modalities together, samples changing with time or stochastic in nature can be studied.

\section{References}

[1] W-S. Chiang, et al., Proceedings of SPWLA 58th Annual Logging Symposium, June 17-21, 2017.

[2] J. M. LaManna, D. S. Hussey, E. Baltic, D. L. Jacobson, Rev. Sci. Inst., submitted (2017).
(A)

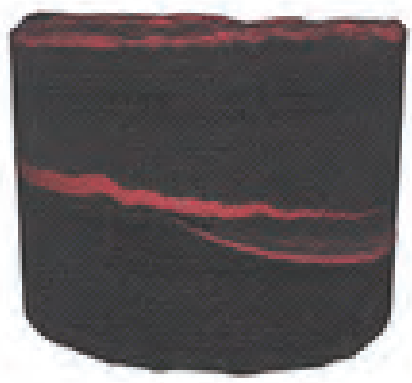

(B)

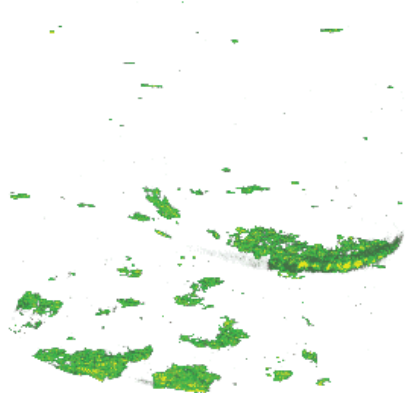

(C)

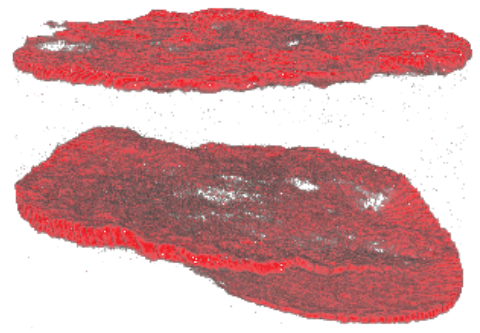

FIGURE 1: (A) Neutron volume rendering of an entire shale sample, (B) Segmented X-ray volume rendering of a shale core showing the distribution of minerals, (C) Segmented neutron volume rendering of a shale core showing the distribution of hydrogen rich organic material. 


\section{Reliability and Availability of the Reactor}

The reactor operated for 228 days during FY 2017, achieving a reliability of $98 \%$. There were three unplanned shutdowns which resulted in 5.6 days lost. Two of the shutdowns were due instrumentation failures in the PLC for the cold source. Some of the lost time was made up by extending the reactor cycles.

\section{New Shim Arms and New Shim Arm Drive Mechanisms}

The NBSR is controlled by four Safety Shim Rods (SSR). These are pipes that are made of thin cadmium sheet ("shim") that are entirely encapsulated in aluminum, so that the cadmium is not exposed to the heavy water reactor moderator. Every four years the SSRs need to be replaced, because the cadmium isotope that is responsible for their capacity to capture neutrons is slowly consumed as the reactor operates. The main purpose of the reactor outage that began in September 2017 is to replace the SSRs with fresh ones. This year in addition to replacing the SSRs we will also replace the shim arm drive mechanisms. As with many other aging reactor components, we find it increasingly difficult to perform maintenance on these systems. The main factors in the risk analysis are obsolescence of spare parts and the time spent inside the shim arm cavities to perform maintenance and repairs. From a mechanical point of view, the present four shim arm drive mechanisms are sufficiently different from each other that each drive requires individual spare part inventories. In addition, the mechanical design is unnecessarily adjustable and complex. Taken all together these conditions present an area where reactor reliability could be improved.

Over the last (8 to 10) years we have designed, tested, and documented a replacement system. From a nuclear regulatory point of view, the SSR drive mechanisms are one of the most scrutinized systems in the entire NBSR. They are, after all, the "brakes" of the reactor. Therefore, the new design is not revolutionary and is almost identical to the existing design. Although based on the old system and very similar to it, the new system is fully engineered from the bottom up, which allows much better use of the spare parts inventory and will allow us to make more quickly make any necessary repairs.

\section{Primary Water Pump System Upgrade}

The primary cooling system of the NBSR is designed to circulate heavy water through the reactor fuel, where it also functions as

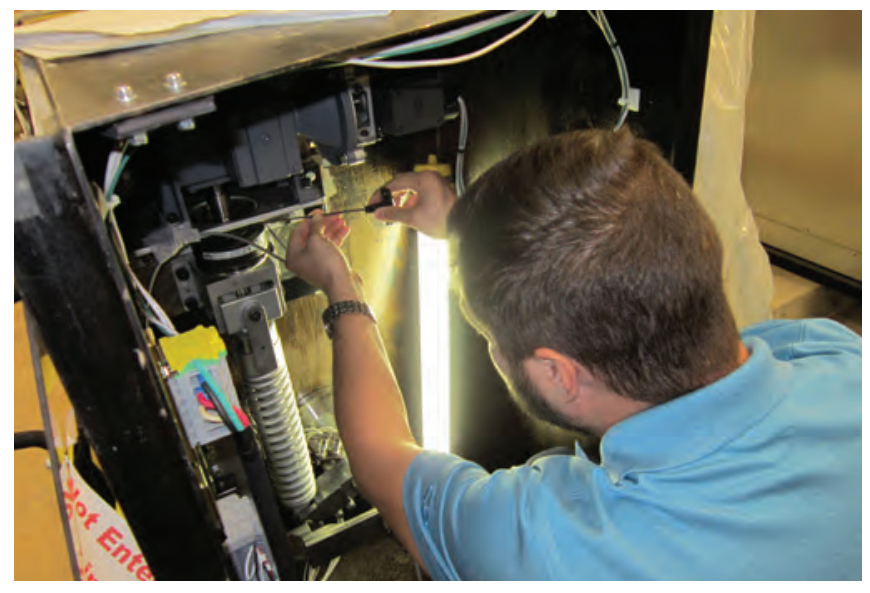

FIGURE 1: Dan Mattes of Reactor Operations and Engineering is adjusting the magnetic coupling that initiates reactor SCRAMs in the newly designed shim arm drive mechanism. The picture shows the mechanism mounted in a test stand that exactly mimics the mechanical behavior of a shim arm as if it were installed in the reactor. Hundreds of SCRAM tests were performed while the mechanical behavior of the assembly was monitored using a high-speed data acquisition system. This allows us to predict adequate mechanical reliability of this assembly for the present maximum predictable useful remaining lifetime of the NBSR ( $>50$ years).

a neutron moderator. Most components of the present primary cooling system date from the time of the original criticality of the NBSR. At present, the check valves associated with the primary pumps are pneumatically operated which require considerable maintenance. Thus, all pumps as well as the present pneumatically operated check valves will be replaced with modern components. The new nozzle check valves are passive valves operated by the process flow. The new check valves are specifically designed to significantly reduce water hammer due to switching from one pump lineup to another (at any given time only three pumps are running: there is one standby pump). This upgrade project started a year ago with a laser interferometry scan of the entire Process Room, which allowed the engineers to precisely map the current plumbing. The new pumps will be placed with respect to those spatially determined boundary conditions. The plumbing suspension system has also been redesigned so that it better allows for thermal contraction and expansion during the running of the reactor. This reduces the mechanical stress on the pump bearings and the pump seals. This new system is expected to be more robust than the existing system, leading to reduced maintenance and improved reliability. 


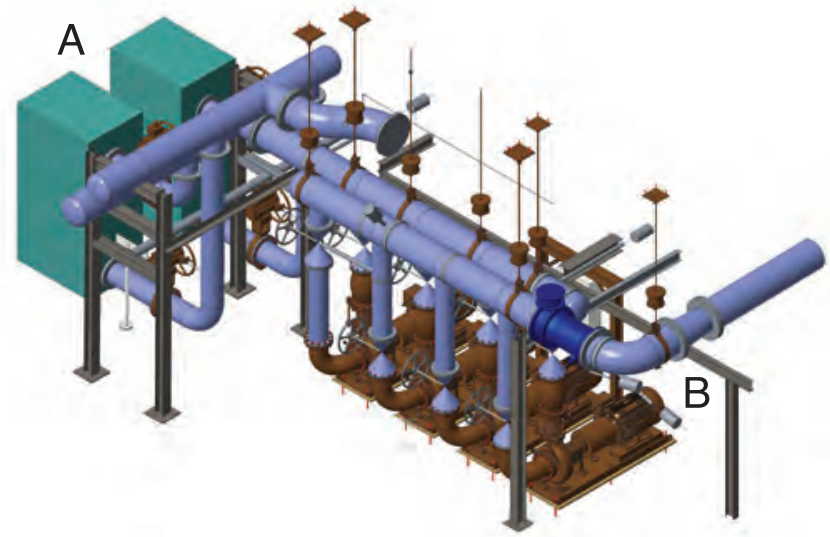

FIGURE 2: Three-dimensional rendering of the primary cooling system upgrade in the Process Room of the NBSR. The cyan colored boxes on the top left are the existing NBSR heat exchangers. The blue structures represent existing plumbing. All the brown items are replacement and/or new parts (pumps, valves and support systems). To get an idea of the size of this assembly, the distance between "A" and " $\mathrm{B}$ " is approximately 12 meters (40 feet).

\section{The Liquid Deuterium Cold Source - Status Update}

The NCNR has been steadily improving its cold neutron facilities for more than 25 years. Monte Carlo simulations have shown that a liquid deuterium $\left(L D_{2}\right)$ source will provide an average gain of 1.5 between $4 \AA$ and $9 \AA$ with respect to the existing liquid hydrogen cold source, and a gain of 2 at the longest wavelengths.

To achieve these gains, a large volume (35 liters) of $\mathrm{LD}_{2}$ is required. The expected nuclear heat load in this moderator and vessel is $4 \mathrm{~kW}$. A new, $7 \mathrm{~kW}$ helium cryogenic refrigerator is being installed to provide the necessary cooling capacity. It was designed to operate at either $14 \mathrm{~K}$, to operate the existing liquid hydrogen sources, or at $17 \mathrm{~K}$ for the future $\mathrm{LD}_{2}$ source. All the major components, compressors, oil removal system, coldbox, $\mathrm{LN}_{2}$ supply, PLC control system and low-pressure helium storage tank, are installed, tested and ready for operation. The final step in the commissioning of the new refrigerator is now underway. The turbine expander will soon be installed and a series of tests in September will measure its cooling capacity as a function of temperature. Upon the successful completion of these tests, the refrigerator will be placed in routine operation in January 2018.

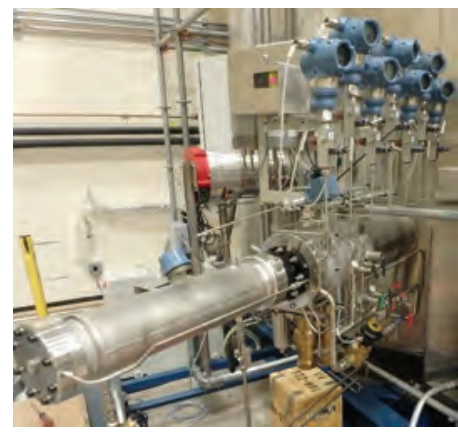

FIGURE 3: The turbine assembly welded to the coldbox. The black flange in the center is a placeholder for the actual turbine cartridge stored in the wooden crate below. In the foreground is the helium brake gas heat exchanger. The turbine floats on $\mathrm{He}$ bearing gas as it spins at a nominal speed of $1560 \mathrm{~Hz}$ (94 krpm).
Most of the major components of the cold source have already been secured. A pair of deuterium condensers were obtained and have since been integrated into two condenser assemblies (one spare) with completion of their vacuum and He containment jackets. The $16 \mathrm{~m}^{3}$ ballast tank was fabricated and installed outside the new guide hall. A contract has recently been awarded for detailed designs of the cryostat assembly components and the fabrication of prototype vessels for testing. Upon successful completion of tests on the prototypes, two complete cryostats will be fabricated.

\section{Future Options for the NCNR Neutron Source}

Reactor Operations and Engineering (ROE) has been studying options for the future of the facility at the request of NIST management as input to the Master Plan for the Gaithersburg campus. This comes as the NBSR is about to celebrate the 50th anniversary of the initial criticality of the NBSR. There were three general options studied:

1. Maintain the NBSR in its current configuration for 50 more years.

2. Upgrade the NBSR to replacing potentially unreliable systems and reconfigure the core to enhance the cold and thermal neutron fluxes.

\section{Replace the NBSR with a new reactor.}

Not studied was the suite of neutron scattering instruments or the neutron guide network, which will always be evolving to meet the needs of the research community. In the near term, NCNR is planning to replace the large liquid hydrogen cold source with a deuterium cold source, and converting the NBSR from high-enriched uranium fuel to low-enriched fuel (LEU) in 2027. Any upgrade or replacement reactor would have to be fueled with LEU. Currently the NBSR is licensed to operate until 2029, and ROE is planning to again renew the license for 20 more years.

Maintain the NBSR current configuration: For Option 1, ROE identified and evaluated the reliability of 17 major systems and estimated the cost of maintaining them. Modifications and upgrades are always ongoing as part of the NBSR Aging Management Program. (For example, the primary cooling system will be upgraded this fall with new pumps and valves.) Although Option 1 is the least expensive, it is expected that the ROE equipment budget will eventually double to about $\$ 4 \mathrm{M}$. While none of the systems presented safety issues or were judged to be in poor health, the Thermal Shield (TS) cooling system is marginal just by its very nature, the reliance of 188 copper tubes buried in concrete to deliver water to it. The major risk associates with Option 1 is the possibility of unplanned shutdowns. There is also little room for expansion. 
Reactor Upgrade: The fact that the NBSR reactor vessel was designed to be replaced, whereas the TS is literally cast in concrete, opens the possibility of installing a new thermal shield inside the original, and a smaller reactor vessel inside it. Monte Carlo simulations demonstrated that $90 \%$ of the energy currently deposited in the existing TS could be captured by an 8-inch-thick, steel inner TS. Neutronics calculations were performed for several possible fuel configurations in a smaller vessel bounded by two cases. Case 1 is similar to the existing core but with the fuel elements on a 6 in pitch rather than a 7 in pitch, cooled with $\mathrm{D}_{2} \mathrm{O}$ and maintaining the thermal neutron flux trap at the mid plane. Case 2 has a very compact core, light water cooled, and surrounded by a $\mathrm{D}_{2} \mathrm{O}$ reflector in a manner similar to several of the more recent research reactor designs. Aside from increased reliability, there is little to be gained from either case. The beam ports and cold sources need to be moved closer to the core just to maintain the existing flux, and interfere with one another in the compact core. To get a $50 \%$ gain, the reactor power must be increased $50 \%$. Such an upgrade would probably take 3 to 4 years to complete and cost $\$ 550 \mathrm{M}$ - $\$ 700 \mathrm{M}$, not including lost productivity.

Replacement Reactor: For the last three years, NCNR has been studying conceptual designs for a new reactor, fueled with LEU and optimized to produce cold neutrons. A horizontally split core with two compact clusters of fuel elements would be surrounded with a $\mathrm{D}_{2} \mathrm{O}$ reflector, and would produce a large thermal neutron flux trap in which two cold sources could be located [1]. With such a configuration, two guide halls are envisioned, so NCNR could double the number of neutron guides and instruments. A new reactor would cost about $\$ 1 \mathrm{~B}$. Ideally it could be built adjacent to the existing guide hall while the NBSR continued to operate until the new reactor is finished. A second guide hall would also be built. Option 3 would make the NCNR a world leader in neutron research and serve the community for the remainder of the 21st Century.

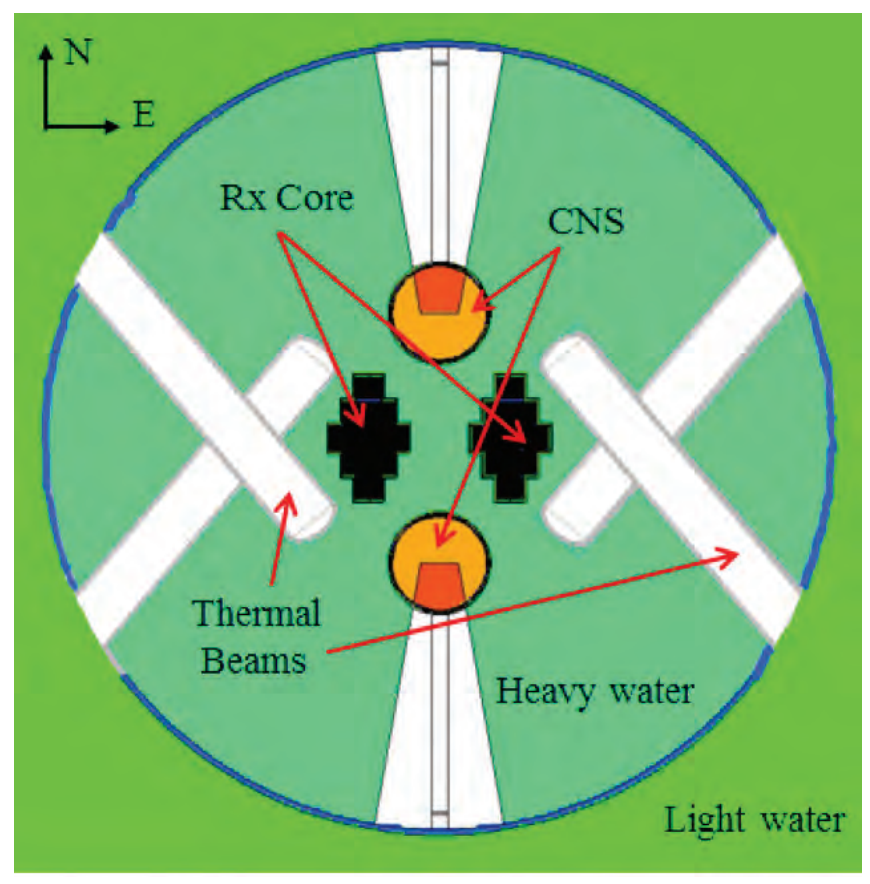

FIGURE 4: Conceptual design for a horizontally split core (Option 3) with two vertical deuterium cold sources. Each core half contains 9 fuel elements cooled with $\mathrm{H}_{2} \mathrm{O}$ and surrounded with a large $\mathrm{D}_{2} \mathrm{O}$ reflector.

\section{Reference}

[1] Z. Wu, R. E. Williams, J. M. Rowe, T. H. Newton, and S. O'Kelly, Nuclear Technology, 199(1), 67 (2017). 


\section{Facility Development}

\section{Installations and Upgrades}

\section{vSANS on NG-3}

The new vSANS instrument saw its first neutrons on August 11 and commissioning has begun in earnest with test data collected and reduced.

Many milestones for the design, procurement, fabrication, installation and testing for vSANS were achieved in 2017. After a contractor delay of more than one year, the three segments that make up the detector vessel arrived at the NCNR on March 13. Each segment is $7.9 \mathrm{~m}$ long with an inside diameter of $2.3 \mathrm{~m}$. NCNR technicians covered the inside of each segment with sheets of flexible boron loaded rubber(bora-flex) neutron shielding. The vessels were then moved to the East Guide Hall to be assembled and aligned to within $0.8 \mathrm{~mm}$. The detector carriages were installed and an issue that prevented the carriages from moving at their top-level specification speed was solved. The detectors were then installed on the front and middle carriages. After this flurry of installation and testing of mechanical and electrical components, the first neutron beam on detector with the vessel in air was on August 11, and under vacuum was on August 15. Tests of background, detector response, calibration, scattering from standard samples, and data reduction was carried out until the scheduled shutdown in September.

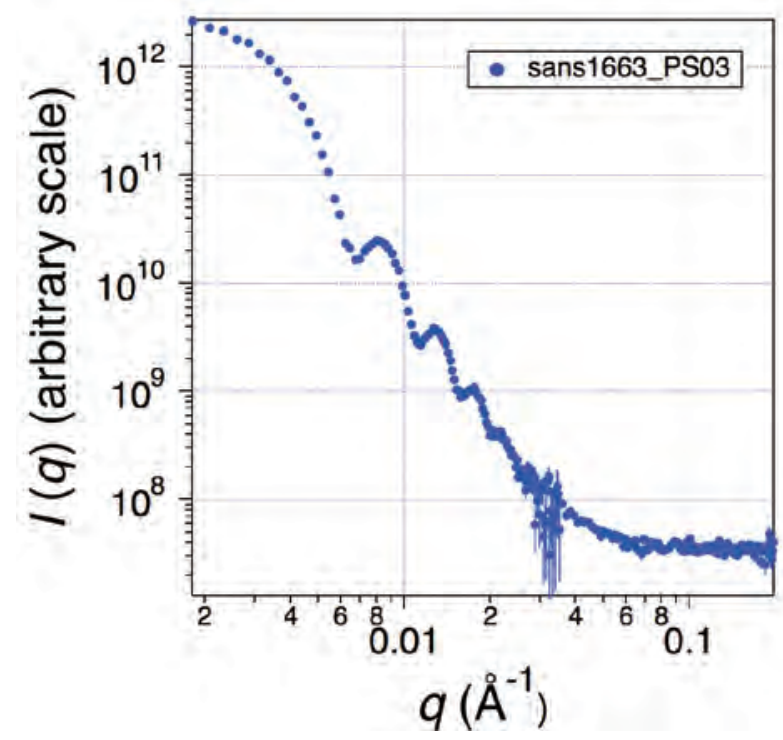

FIGURE 1: One of the initial data sets from the vSANS instrument. The middle carriage was set at a distance of $19 \mathrm{~m}$ from the sample, and the front carriage at $4 \mathrm{~m}$. The sample is latex spheres of radius $\approx 70 \mathrm{~nm}$.
Additional features are still to be incorporated into vSANS. For example, the 2D high-resolution detector for the rear carriage is on site and has been tested and accepted. Installation of this detector is targeted for December 2017, but some additional components are still required for it to be operational. Starting with the first reactor cycle of 2018, commissioning of the instrument will continue using the instrument in SANS mode. By March 2018 we anticipate some in-house science experiments to be possible and user proposals have been solicited on a limited basis in the most recent Call for Proposals.

\section{CANDOR}

The construction of the CANDOR instrument has been delayed due to the realization that the thermal diffuse scattering (TDS) of neutrons by the analyzer crystals would pose a significant challenge to data reduction. This is because the TDS causes the neutrons to appear in incorrect detectors. While this could, in principle, can be calculated and corrected for in data reduction, the adjustment could in some cases be a substantial fraction of the total counts in an individual detector. Thus, simulations and design studies are underway to incorporate cryogenic cooling of the HOPG into the detector package. We anticipate that a two-array prototype will be constructed and tested with first neutrons on detector approximately in September 2018. The first specular reflection experiment with the sample area installed, basic NICE data acquisition software features tested, and basic data reduction software built and tested are all targeted for January 2019.

In the meantime, beamline components continue to arrive and are being installed at NG1. Much of the pre-sample optics has been completed, including the $\mathrm{X}$ deflector. Components for polarized beam operation are also being included with the double $\mathrm{V}$ polarizer in place and testing of the RF flipper well advanced. Installation of the primary instrument and the sample area is planned for the spring and summer of 2018, allowing the testing of NICE to begin.

Development of the detector continues with contracts and procurements now in place to support the construction of the detector assembly consisting of about 30 detector arrays. A single CANDOR detector array consist 54 highly oriented pyrolytic graphite crystals set at different takeoff angles to diffract neutrons of into ${ }^{6} \mathrm{LiF}: \mathrm{ZnS}(\mathrm{Ag})$ proportional counters. The NCNR has worked with the scintillator manufacturer to refine the construction of the ultrathin proportional counters 


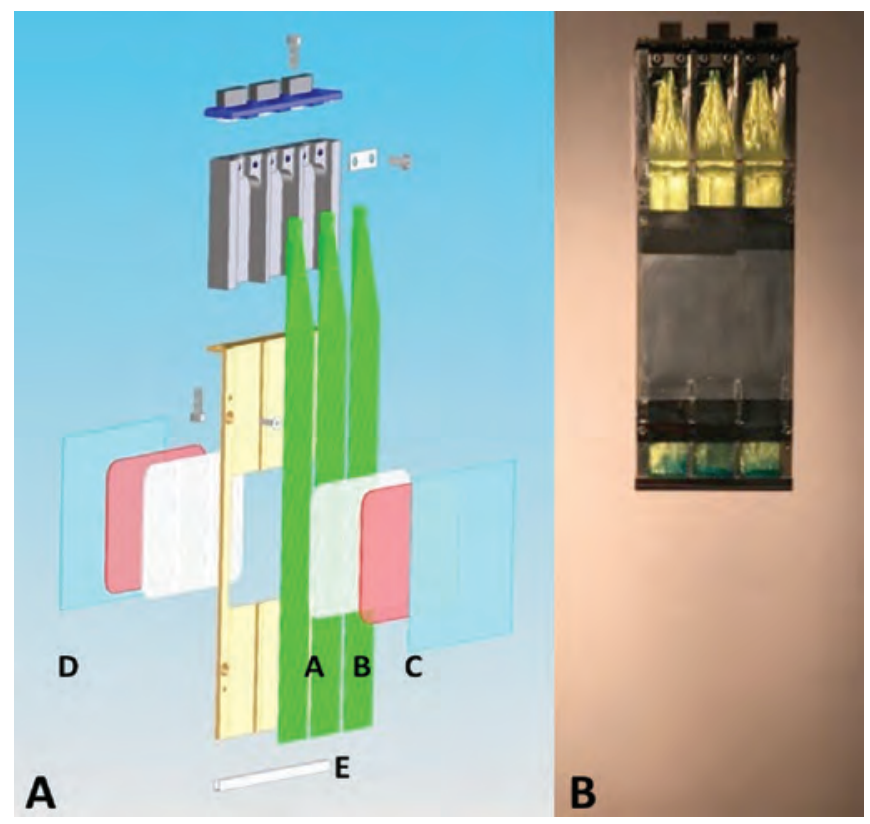

FIGURE 2: Exploded assembly diagram and photograph of the triple frame neutron proportional counter. (A) Exploded assembly of triple frame. Layer A consists of 1:2:0.6 Ni-doped scintillator "primer". Layer B is a slab of 1:2:0.14 stock scintillator. Layer $C$ is the mirrored aluminum reflector on the leading face of the detector. Layer $\mathrm{D}$ is the mirrored plastic reflector on the trailing face of the detector. The silicon photomultiplier photosensors are in the printed circuit board mounted to the top of the assembly. (B) is a photograph of a fully assembled counter.

for mass production. NIST has also contracted with a highspeed digitizer manufacturer to develop real time pulse shape discrimination electronics for the entire detector assembly (projected to include more than 1500 individual detectors). The overall proportional counter performance is a combination of many factors including the thickness of the sensor, the arrangement of wavelength shifting fibers, the photodetection efficiency of the solid-state photo sensor, and the algorithm used for neutron/gamma discrimination. Detectors have been characterized against gold foil irradiation and calibrated flux monitors. These measurements have demonstrated that $1.5 \mathrm{~mm}$ thick proportional counters with $93 \%$ absolute efficiency for $3.27 \mathrm{meV}$ neutrons and gamma rejection on the order of $10^{-7}$ can be manufactured reproducibly.

\section{Engineering Diffractometer}

Design, fabrication and installations continue at BT-8 to further improve instrument performance. A new monochromator has been procured with expected delivery in January 2018, and a new detector with higher efficiency and was installed in August 2017. The current detector housing is being redesigned to fully utilize the larger angular coverage of the new detector. Together, these improvements will result in a gain of a factor of 20 . Also, nearly all components for Octo-Strain II have been procured, allowing it to be assembled, tested, and ready for BT-8 in early 2018.
Octo-Strain II will have the capability to apply a load three times larger than before, a capability necessary for measurements of today's high-strength steels.

\section{Backscattering Spectrometer}

When the Backscattering Spectrometer was built, it benefitted from the best focusing guide technology available. However, neutron optics has advanced considerably and the simple linear converging guide was no longer state-of-the-art. Thus, the Center for High Resolution Neutron Scattering chose to replace the incident beam optics with a converging guide having modern supermirror coatings and a more advanced profile than the straight taper. The installation of this new $4 \mathrm{~m}$ section of guide, completed in August 2017, resulted in intensity gain of a factor of 1.9. The neutron flux at the sample position is currently approximately $6 \times 10^{5} \mathrm{n} / \mathrm{cm}^{2}$-sec. The energy resolution remains $\approx 0.8 \mu \mathrm{eV}$.

\section{Building 321}

Thanks to NIST, building 321, located at the south end of the NCNR site, was completed and NCNR took occupancy in August 2017. This climate-controlled storage building gives the NCNR much-needed space to store instrument components, shielding, and components for future developments such as the $D_{2}$ cold source replacement.

\section{New DCS Mezzanine}

The installation of the vSANS scattering chamber required extending the DCS mezzanine to span the new, considerably larger tank. The NCNR took advantage of this necessary change to address the facility's need for additional space for sample environment equipment by building a larger mezzanine that spans the entire Disc Chopper Spectrometer (DCS) scattering chamber as well. The new $77 \mathrm{~m}^{2}$ mezzanine provides ample room not only for DCS sample environments, storage cabinets, and an area for sample preparation, it is also the permanent home of the $10 \mathrm{~T}$ superconducting magnet.

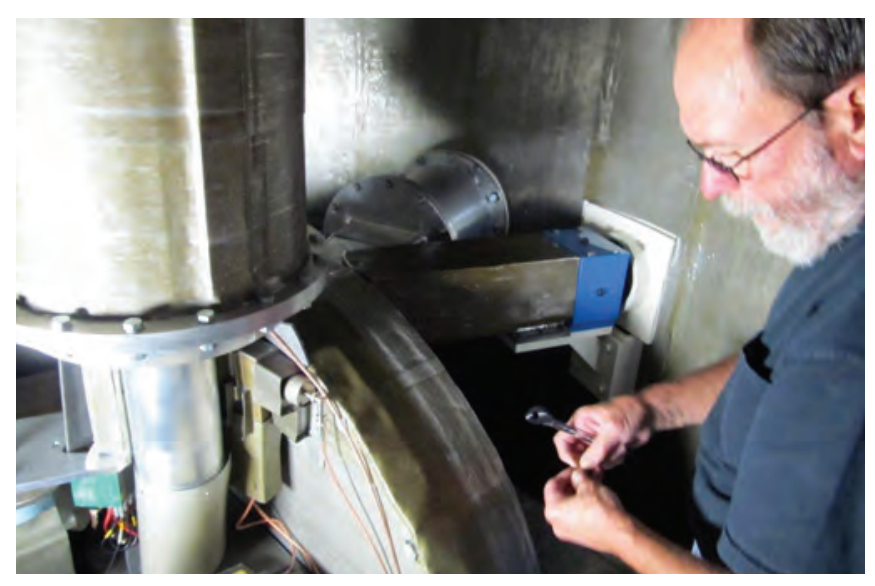

FIGURE 3: Scott Slifer works on the upgrade of the NG-2 Backscattering instrument. 


\section{New Guide Hall Laboratory}

A new user laboratory is now open in the northwest corner of the NCNR Guide Hall. The new laboratory provides space for limited handling of samples and sample holders that may be slightly radioactive without the need for removing them from the Guide Hall. In addition to the laboratory supplies commonly stacked in NCNR user laboratories, it is equipped with a fume hood that can be used to handle both activated samples and dispersible nanoparticles. All waste from the laboratory is handled as low-level radioactive waste to ensure that there is no uncontrolled release from the facility. In addition, the lab has its own ventilation system and fire suppression system.

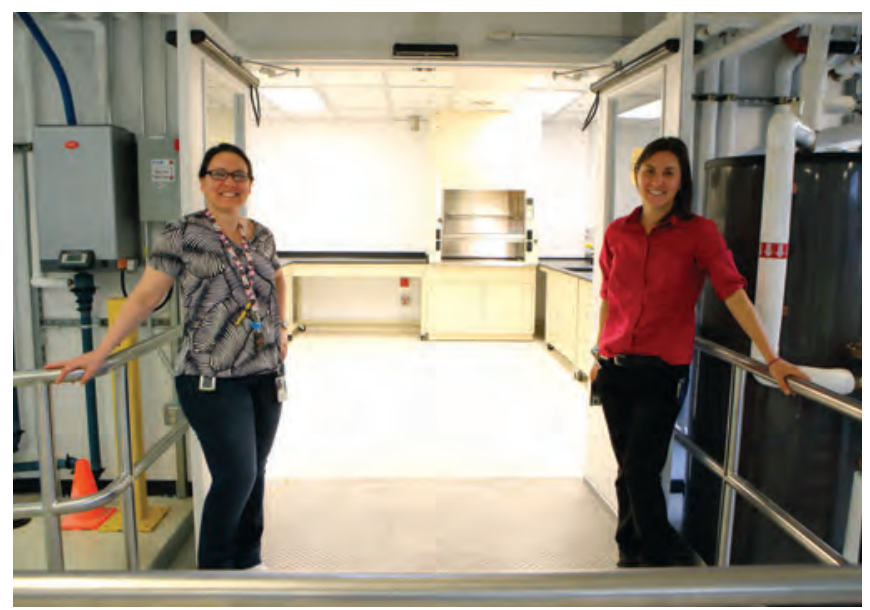

FIGURE 5: Yamali Hernandez and Mikala Shremshock welcome users to the new Guide Hall laboratory.

\section{Helium Spin Filters}

The NCNR supports an active program to provide state-of-the-art neutron polarization capabilities. Measurements using polarized neutron are routinely carried out on the BT-7 thermal neutron triple-axis spectrometer, the NG-7 30 m small-angle neutron scattering instrument, and the Multi-Axis Crystal Spectrometer (MACS). During the past year, the NCNR's spin filter program served 39 experiments, for a total of 117 days of beam time, 148 bar-liters of polarized ${ }^{3} \mathrm{He}$ gas, and 96 polarized ${ }^{3} \mathrm{He}$ cells.

Both vSANS and CANDOR require ${ }^{3}$ He spin filters for all measurements involving polarization analysis of the scattered beam. Thus, the NCNR is developing ${ }^{3} \mathrm{He}$ spin analyzers and is optimizing the magnetically shielded solenoids necessary to maintain the ${ }^{3} \mathrm{He}$ polarization. We will be use a ${ }^{3} \mathrm{He}$ adiabatic-fastpassage, NMR-based neutron spin flipper for the scattered beam. The NCNR is also optimizing radio-frequency neutron spin flippers that will be used in conjunction with a double $V$ supermirror polarizer (efficiency > $95 \%$ ), that will be utilized in the incident beam of both instruments.

The NCNR has improved the ${ }^{3} \mathrm{He}$ nuclear magnetic resonance software so that it could be seamlessly integrated into the NICE software on SANS, reflectometers, and the test station for all polarized beam experiments. It will also be incorporated the NICE data acquisition software for vSANS, CANDOR, and MACS. NIST is also developing an in-situ ${ }^{3} \mathrm{He}$ polarizer and an ex-situ ${ }^{3} \mathrm{He}$ analyzer for polarized neutron imaging on the NG-6 cold neutron imaging facility. 


\section{Data Acquisition Software}

The New Instrument Control Environment (NICE) is a Java-based data acquisition package that incorporates a highly versatile scripting capability to enable end user adaptations to be coded under all the major scientific programming languages. This year, much of the effort has focused on the two instruments in development, vSANS and CANDOR. As of August, NICE has been used to collect data on the vSANS instrument, writing data in a Nexus-compliant format. NICE will be running the instrument in January 2018 as the instrument enters the user program. For CANDOR, nearly all of the NICE module has been written. This includes a new system for setting up generalized reflectometry experiments where a user can create an entire series of measurements by entering a few pieces of information about their sample and the type of measurement to perform. This system even allows the user to enter the wall clock time and how long they would like the experiment to last, allowing NICE to calculate the optimal set of measurements to fit the available time. These capabilities will be rolled out on all reflectometers in the building, including CANDOR. There were also improvements focusing on time estimation and planning. The system for "dry running" a measurement was completely replaced with a more streamlined version which executes many times faster and provides highly detailed and accurate account of the run sequence timing. Plans are in the works provide a live time estimate for all instrument operations which updates as the user changes their planned measurement and as time passes.

\section{Data Analysis Software}

Funded jointly by the NSF and EPSRC, the CCP-SAS project is focused on developing an easy-to-use open-source modeling package that enables users to generate physically accurate atomistic models, calculate scattering profiles and compare results to experimental scattering data sets in a single web-based software suite. SASSIE-web is now available to the international scattering community. Workshop and training sessions at scattering centers and scientific meetings have expanded outreach and impact. The implementation of SASSIE-web on several domestic and international high-performance supercomputing centers (HPC) has been prototyped. To date, over forty-three manuscripts using our software products on a variety of structural biology and soft-matter problems have been published. With over five hundred registered users, the goals of the next year are to extend and enhance the user experience and fully implement HPC deployments world-wide.

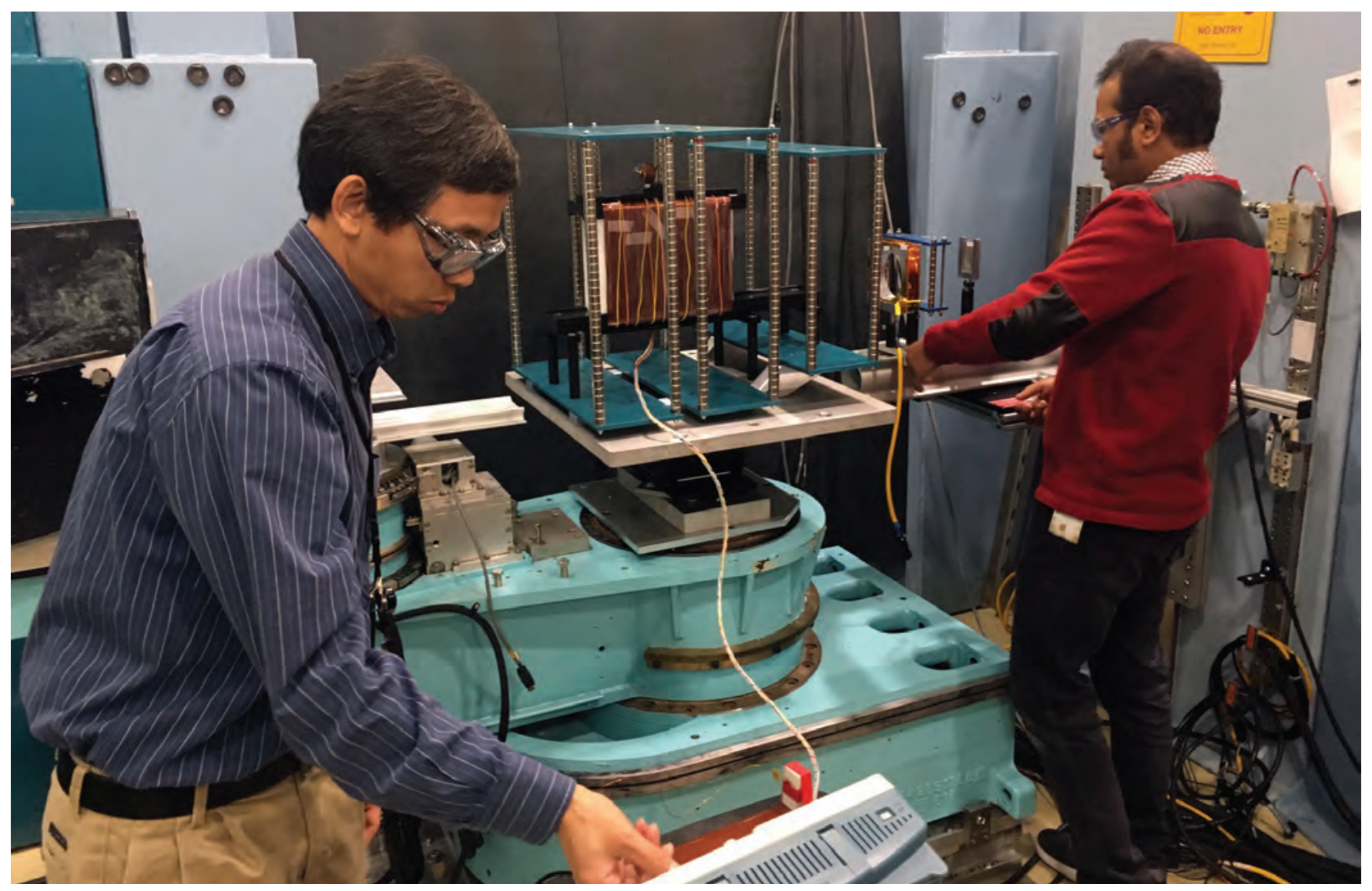

FIGURE 6: Wangchun Chen and Md Taufique Hassan performing tests on the RF spin flipper design for CANDOR. 


\section{Serving the Science and Technology Community}

$\mathrm{T}$ he mission of the NIST Center for Neutron Research is to assure the availability of neutron measurement capabilities to meet the needs of U.S. researchers from industry, academia and other U.S. government agencies. To carry out this mission, the NCNR uses several different mechanisms to work with participants from outside NIST, including a competitive proposal process, instrument partnerships, and collaborative research with NIST.

\section{Proposal System}

Most of the beam time on NCNR instruments is made available through a peer-review proposal process. The NCNR issues calls for proposals approximately twice a year. Proposals are reviewed at several different levels. First, several different expert external referees evaluate each proposal on merit and provide us with written comments and ratings. Second, the proposals are evaluated on technical feasibility and safety by NCNR staff. Third, we convene our Beam Time Allocation Committee (BTAC) to assess the reviews and to allocate the available instrument time. Using the results of the external peer review and their own judgment, the BTAC makes recommendations to the NCNR Director on the amount of beam time to allocate to each approved experiment. Approved experiments are scheduled by NCNR staff members in consultation with the experimenters.

The current BTAC members are:

- Pinar Akcora (Stevens Institute of Technology)

- Andrew Allen (NIST Ceramics Division)

- Jeffrey Allen (Michigan Technological University)

- Collin Broholm (The Johns Hopkins University)

- Leslie Butler (Louisiana State University)

- Thomas Epps (University of Delaware)

- Kushol Gupta (University of Pennsylvania)

- Valery Kiryukhin (Rutgers University)

- Ramanan Krishnamoorti (University of Houston)

- Jennifer Lee (National Institutes of Health)

- Raul Lobo (University of Delaware)
- Steven May (Drexel University)

- Martin Mourigal (Georgia Institute of Technology)

- Lilo Pozzo (University of Washington)

- Carlos Rinaldi (University of Florida)

- Gila Stein (University of Tennessee)

\section{Partnerships}

The NCNR may form partnerships with other institutions to fund the development and operation of selected instruments. Partnerships are negotiated for a fixed period and may be renewed if there is mutual interest and a continued need. These partnerships have proven to be an important and effective way to expand the research community's access to NCNR capabilities.

\section{Collaboration with NIST}

Some time on all instruments is available to NIST staff to support NIST's mission. This time is used to work on NIST research needs, instrument development, and promoting the widespread use of neutron measurements in important research areas, particularly by new users. As a result of these objectives, a significant fraction of the time available to NIST staff is used collaboratively by external users, who often take the lead in the research. Access through such collaborations is managed through written beam time requests. In contrast to proposals, beam time requests are reviewed and approved internally by NCNR staff. We encourage users interested in exploring collaborative research opportunities to contact an appropriate NCNR staff member.

\section{Research Participation and Productivity}

The NCNR continued its strong record of serving the U.S. research community this year. Over the 2017 reporting year, the NCNR served 2769 researchers. (Research participants include users who come to the NCNR to use the facility as well as active collaborators, including co-proposers of approved experiments, and co-authors of publications resulting from work performed at the NCNR.) As the number of participants has grown, the number of publications per year has also increased. 


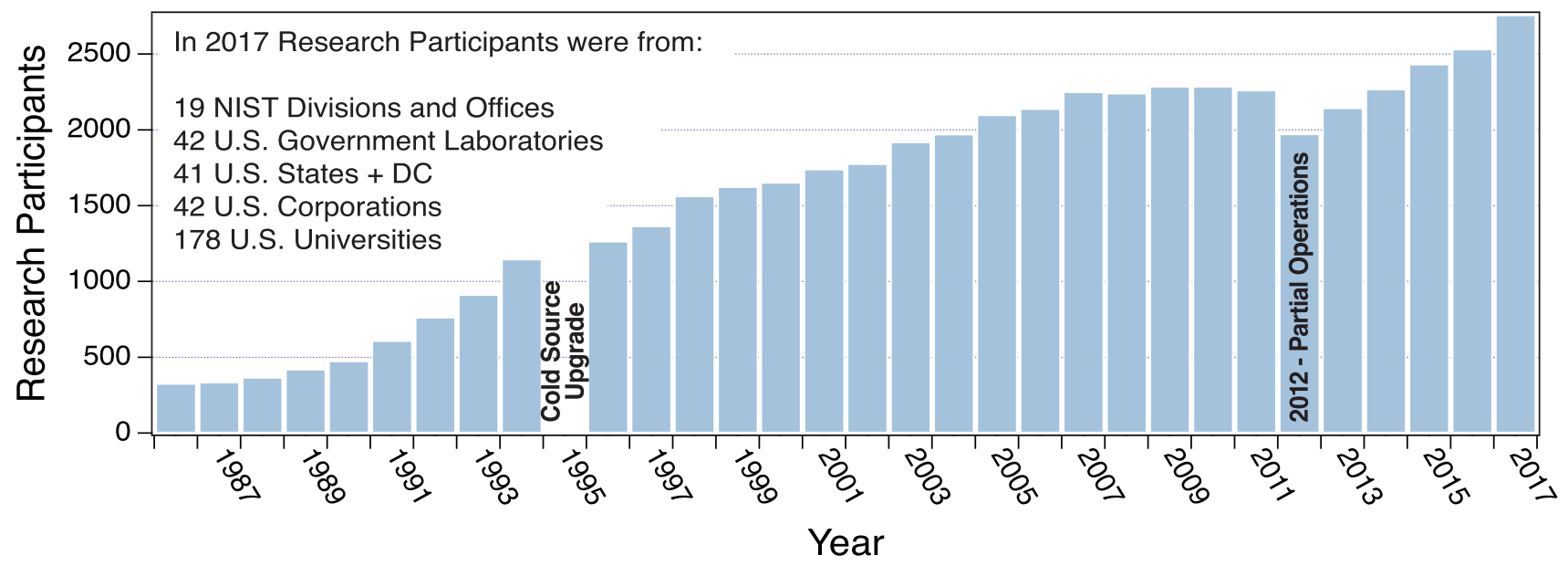

FIGURE 1: Research participants at the NCNR 1986 - 2017.

\section{NCNR Proposal Program}

In response to the last two calls for proposals (calls 35 and 36) for instrument time, we received 661 proposals, of which 322 were approved and received beam time. For the most recent call, the oversubscription, i.e., the ratio of days requested on all proposals to the days available, was 2.5 on average. The following table shows the data for several instrument classes.

\begin{tabular}{lccc} 
Instrument class & Proposals & $\begin{array}{c}\text { Days } \\
\text { requested }\end{array}$ & $\begin{array}{c}\text { Days } \\
\text { allocated }\end{array}$ \\
SANS and USANS & 237 & 803 & 353 \\
Reflectometers & 100 & 648 & 272 \\
Spectrometers & 265 & 1833 & 617 \\
Diffraction & 34 & 93 & 52 \\
Imaging & 25 & 141 & 97 \\
Total & $\mathbf{6 6 1}$ & $\mathbf{3 5 1 8}$ & $\mathbf{1 3 9 1}$ \\
\hline
\end{tabular}

\section{Users Group}

The NCNR Users Group (NUG) provides an independent forum for all facility users to raise issues to NCNR management, working through its executive officers to carry out this function. The current members of the NUG Executive Committee are Megan Robertson (University of Houston), Michael Crawford (University of Delaware), Julie Hipp (University of Delaware, student/postdoc member), Carlos López-Barrón (ExxonMobil), Dmitry Reznik (University of Colorado), Rafael Verduzco (Rice University), and Igor Zaliznyak (Brookhaven National Laboratory).

The NUG conducted a user survey in the fall of 2015. There were more than 450 responses. Overall the results, which are posted on the NUG website (www.indiana.edu/ lens/nug/nug.php), showed improvement relative to those from 2011 in 5 of the 6 general categories. During the past year, we discussed the survey results with the NUG executive committee. Working closely with NUG, the
NCNR and CHRNS management teams developed a comprehensive response/action plan designed to make the user experience more productive and enjoyable (Refer to ncnr.nist.gov/news/Response_ to_survey_FINAL_19jul2016.pdf).

\section{Panel of Assessment}

The major organizational components of NIST are evaluated for quality and effectiveness by the National Research Council (NRC), the principal operating agency of both the National Academy of Sciences and the National Academy of Engineering. A panel appointed by the NRC convened at the NCNR on July 7-8, 2015. Their findings are summarized in a published report, "An Assessment of the National Institute of Standards and Technology Center for Neutron Research: Fiscal Year 2015," which is available at https://www.nap.edu/catalog/21878/ an-assessment-of-the-national-institute-of-standards-andtechnology-center-for-neutron-research. The panel members included Peter Green (University of Michigan, chair), Frank Bates (University of Minnesota), Bruce Gaulin (McMaster University), Janos Kirz (Lawrence Berkeley National Laboratory), V. Adrian Parsegian (University of Massachusetts Amherst), Sunhil Sinha (University of California at San Diego). A new panel will convene at the NCNR in 2018.

\section{The Center for High Resolution Neutron Scattering (CHRNS)}

CHRNS is a national user facility that is jointly funded by the National Science Foundation and the NCNR. Its primary goal is to maximize access to state-of-the-art neutron scattering instrumentation for the academic research community. It operates five neutron scattering instruments at the NCNR, enabling users from around the nation to observe dynamical phenomena involving energies from $\approx 30 \mathrm{neV}$ to $\approx 10 \mathrm{meV}$, and to obtain structural information on length scales from $\approx 1 \mathrm{~nm}$ to $\approx 10 \mu \mathrm{m}$. A more detailed account of CHRNS activities may be found on pp 65 of this report. 


\section{Partnerships for Specific Instruments}

\section{NG-7 SANS Consortium}

A consortium that includes NIST, the ExxonMobil Research and Engineering Company, and the Industrial Partnership for Research in Interfacial and Materials Engineering (IPRIME) led by the University of Minnesota, operates, maintains, and conducts research at the $30 \mathrm{~m}$ SANS instrument located on NG7. Twenty-five percent of the beam time on this instrument is allocated to the general scientific community through the NCNR's proposal system. Consortium members conduct inde-pendent research programs primarily in the area of large-scale structure in soft matter. For example, ExxonMobil has used this instrument to deepen their understanding of the underlying nature of ExxonMobil's products and processes, especially in the fields of polymers, complex fluids, and petroleum mixtures.

\section{The nSoft Consortium}

Formed in August 2012, the nSoft Consortium allows member companies to participate with NIST in the development of advanced measurements of materials and manufacturing processes, and develop their own expertise in state-of-the-art measurement technologies to include in their analytical research programs. nSoft develops new neutron-based measurement science for manufacturers of soft materials including plastics, composites, protein solutions, surfactants, and colloidal fluids. Members receive access to leading expertise and training support in neutron technology and soft materials science at NIST. Contact: Ron Jones, nSoft Director, rljones@nist.gov, 301-975-4624.

\section{NIST / General Motors - Neutron Imaging}

An ongoing partnership and collaboration between General Motors and NIST, which also includes Honda Motors through GM's partnership with Honda, continues to yield exciting results using neutron imaging. Neutron imaging has been employed to visualize the operation of fuel cells for automotive vehicle applications. Neutron imaging is an ideal method for visualizing hydrogen, the fuel of electric vehicle engines. These unique, fundamental measurements provide valuable material characterizations that will help improve the performance, increase the reliability, and reduce the time to market introduction of the next generation electric car engines. $25 \%$ of the time on the BT-2 Neutron Imaging Facility is made available to the general scientific community through peer-reviewed proposals.

\section{Interagency Collaborations}

The Smithsonian Institution's Nuclear Laboratory for Archaeological Research is part of the Anthropology Department at the National Museum of Natural History. It has had a longstanding and productive partnership with the NCNR, during which time it has chemically analyzed over 43,100 archaeological artifacts by Instrumental Neutron Activation Analysis (INAA), drawing extensively on the collections of the Smithsonian, as well as on those of many other institutions in this country and abroad. Such chemical analyses provide a means of linking these diverse collections together in order to study continuity and change involved in the production of ceramic and other artifacts.

The Center for Food Safety and Applied Nutrition, U.S. Food and Drug Administration (FDA), maintains laboratory facilities at the NCNR providing agency-wide analytical support for food safety and food defense programs. Neutron activation and low-level gamma-ray detection techniques yield multi-element and radiological information about foods and related materials and provide a metrological foundation for FDA's field investigations and for radiological emergency response planning. 


\section{The Center for High Resolution Neutron Scattering (CHRNS)}

$\mathrm{T}$ he Center for High Resolution Neutron Scattering is a national user facility that is jointly funded by the National Science Foundation through its Division of Materials Research (grant number DMR-1508249) and by NIST. The CHRNS agreement was recently renewed for five years beginning on September 1, 2015. The primary purpose of this partnership is to maximize access for the scientific community to transformative neutron scattering instrumentation. The core mission of CHRNS is fourfold: (i) to develop and operate neutron scattering instrumentation, with broad application in materials research, for use by the general scientific community; (ii) to promote the effective use of the CHRNS instruments by having an identifiable staff whose primary function is to assist users; (iii) to conduct research that advances the capabilities and utilization of CHRNS facilities; and (iv) to contribute to the development of human resources through educational and outreach efforts. The scientific community provides essential input for new directions for CHRNS through a variety of mechanisms including user surveys, the most recent of which was administered by the NCNR User Group in the fall of 2015. A new 2 1 1 2 minute video, Getting Great Data with CHRNS, highlights and summarizes CHRNS' focus on advancing neutron scattering measurement capabilities and its prominent role in expanding, educating, and diversifying the community of researchers who use neutron methods. View the video on the NCNR website at https://www.ncnr.nist.gov/staff/dimeo/CHRNS_ Animation_Final.mp4.

\section{Scattering Instruments}

The portfolio of instruments supported by CHRNS will continue to evolve to ensure that the scientific capabilities exceed or are comparable to the best worldwide. During FY 2017, CHRNS supported operation of the NGB 30 m Small Angle Neutron Scattering (SANS) instrument, the Ultra-Small Angle Neutron Scattering (USANS) instrument, the Multi-Angle Crystal Spectrometer (MACS), the High Flux Backscattering Spectrometer (HFBS), and the Neutron Spin-Echo (NSE) spectrometer. Combined, CHRNS instruments can provide structural information on a length scale of $\approx 1 \mathrm{~nm}$ to $\approx 10 \mu \mathrm{m}$, and dynamical information on energy scales from $\approx 30 \mathrm{neV}$ to $\approx 10 \mathrm{meV}$.

The CHRNS agreement continues programs in instrument operations and development on HFBS, NSE, and MACS which boasts the world's highest monochromatic cold-neutron flux and is now the premier instrument in the world for investigations of quantum magnetism. Upgrades of these instruments during the past year include replacement of the incident beam optics on HFBS with a converging guide with modern supermirror coatings and a more advanced profile than the previous straight taper. This improvement increased the flux at the sample position by nearly $2 x$. On NSE, efforts in tuning the instrument have resulted in a $25 \%$ increase of the maximum field integral with a proportional increase of the maximum Fourier time at a given wavelength.

As part of the new CHRNS agreement, two new instruments, vSANS and CANDOR, will be folded into CHRNS as the installation and commissioning of these innovative instruments progress. The data rates provided by the multiplex detector assembly of the CANDOR reflectometer will exceed those available elsewhere in the world by perhaps an order of magnitude or more. The versatile vSANS instrument will cover extensive nano-to-meso length scales in a single measurement, in many cases eliminating the need for combined experiments on USANS and SANS. USANS and SANS will thus be phased out of CHRNS following the commissioning of vSANS and CANDOR. Commissioning of the vSANS instrument has begun with test data collected and reduced. While some features are still being added, user proposals were solicited on a limited basis in the most recent Call for Proposals which had a deadline in November 2017.

\section{Research}

The wide ranges of instrument capabilities available in CHRNS support a very diverse scientific program, allowing researchers in materials science, chemistry, biology, geosciences, and condensed matter physics to investigate materials such as polymers, metals, ceramics, magnetic materials, colloids, fluids and gels, rocks, and biological molecules.

The research community can obtain access to the state-of-theart CHRNS instrumentation using the NCNR's proposal system. Proposals to use the CHRNS instruments are critically reviewed on the basis of scientific merit and/or technological importance. In the previous Call for Proposals (call 36), 210 proposals requested CHRNS instruments, and 90 of these proposals received beam time. Of the 1235 days requested for the CHRNS instruments, 384 were awarded. (These numbers include all of the SANS proposals: Approximately $75 \%$ of the beam time approved by BTAC will be run on the CHRNS NGB SANS.) Roughly half of the users of neutron scattering techniques at the NCNR use CHRNS-funded instruments, and approximately one third of NCNR publications (see the "Publications" section on p. 71), over the current one-year period, are based on research performed using these instruments. This report contains several highlights of CHRNS publications. See the labeled highlights in the table of contents. 


\section{Scientific Support Services}

CHRNS provides scientific support in three critical areas: sample environment, chemical laboratories and the production and delivery of polarized neutron beams. The accomplishments in each of these areas during FY 2017 are summarized below.

The laboratory staff continues to equip and maintain user laboratories and routinely assists users with sample preparations. The staff ensures that users have the tools, supplies and training they need for a successful experiment. In FY 2017 the new Guide Hall Laboratory was opened for business. This laboratory provides a safe environment for CHRNS users to handle activated samples. In addition, a new glove box for the handling of air-sensitive, activated samples was installed. During the past year, CHRNS has added a large capacity balance and a Nanodrop that will allow users to perform UV-Vis measurements for sample quantities as small as $0.5 \mu \mathrm{l}$.

The CHRNS Sample Environment team provides users with the equipment and training needed to make neutron measurements under external conditions of temperature, pressure, magnetic field, humidity, and fluid flow. From mK dilution refrigeration systems to a $1600{ }^{\circ} \mathrm{C}$ furnace, the equipment spans a large temperature range. Beyond precise temperature control, CHRNS users have access to a variety of flow systems, rheometers,

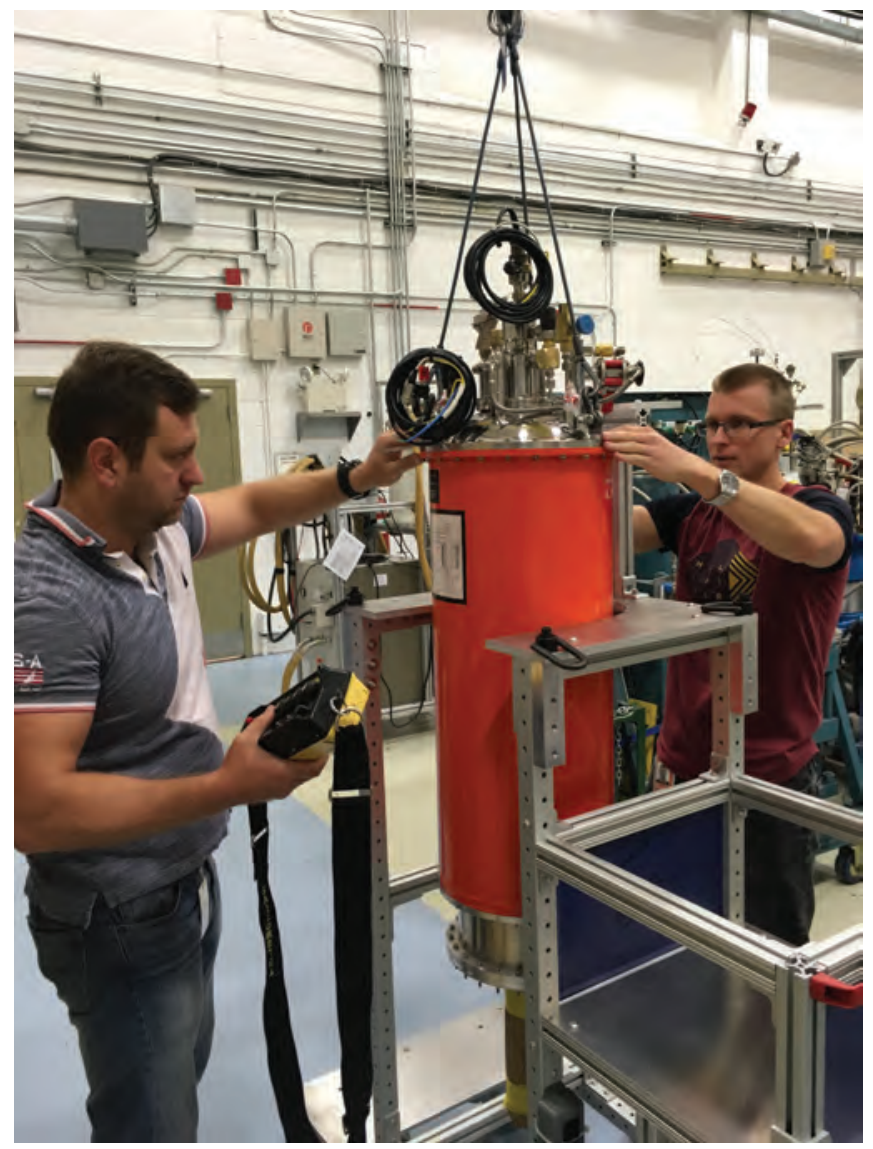

FIGURE 1: CHRNS staff members Sergiy Gladchenko and Yegor Vekhov unpack the new orange cryostat for MACS.

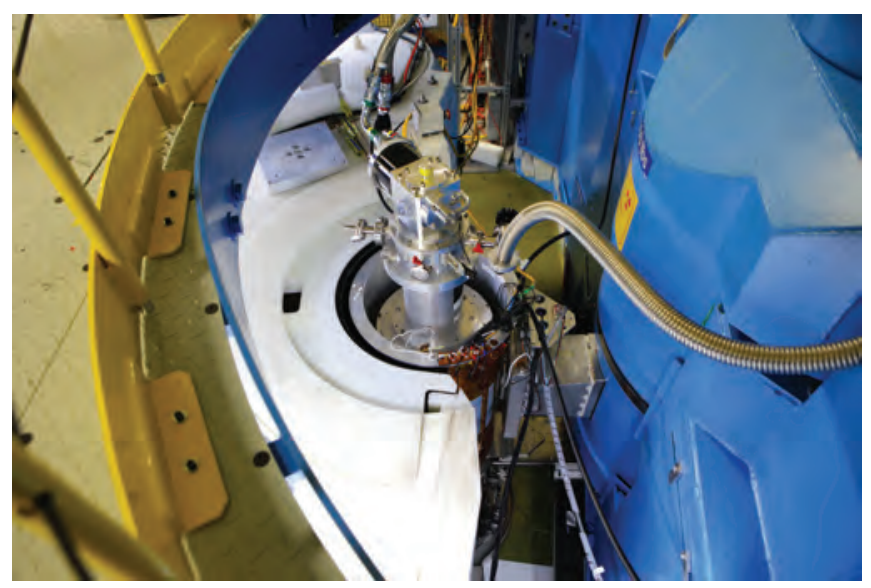

FIGURE 2: Test setup of the ${ }^{3} \mathrm{He}$ horseshoe shells on MACS.

gas-loading systems, two superconducting magnets with fields of up to 11 Tesla, and other complex equipment to control parameters such as pressure, humidity, and electric fields. During FY 2017 the team set up and operated a diverse range of complex sample environment equipment for 172 experiments on CHRNS instruments. This total includes 25 experiments that required access to $\mathrm{mK}$ temperatures, some of which simultaneously required a $11.5 \mathrm{~T}$ magnetic field. In addition, CHRNS is currently commissioning a new orange cryostat for MACS with a specially designed tail that is more compatible with the use of ${ }^{3} \mathrm{He}$ spin filters. MACS users will soon be able to perform polarization experiments in the $\mathrm{mK}$ regime.

CHRNS also provides ${ }^{3} \mathrm{He}$ spin filters that allow fully-polarized neutron scattering measurements to users of the MACS and SANS instruments. Currently, both instruments make frequent use of record-breaking ${ }^{3} \mathrm{He}$ spin analyzer cells with specialized geometries. It is also notable that CHRNS runs the only SANS program in the U.S. that routinely operates with full polarization analysis, and MACS provides the most intense polarized cold neutron beam with the largest scattering angle coverage for polarization analysis. From August 2016 through July 2017, the ${ }^{3} \mathrm{He}$ Spin Filter team serviced a range of user experiments on both MACS and SANS. The team has also focused on deploying infrastructure on MACS that simplifies the use of wide angle neutron polarization analysis. Reliable demagnetization procedures developed previously and recent optimization of an end-compensated solenoid have improved ${ }^{3} \mathrm{He}$ relaxation times on MACS. Such advancements have resulted in ${ }^{3} \mathrm{He}$ relaxation times on MACS close to the intrinsic values observed off-line, and allow the users to perform polarized beam experiments for more than 3 days without swapping cells. In addition, two horseshoe-shaped wide-angle cells have been developed and are now available for routine polarized MACS experiments. These improvements have resulted in a publication in Nature Physics [13, 638 (2017)] highlighting the first use of the wide-angle polarization analysis capability for measuring the Higgs amplitude mode in a two-dimensional antiferromagnet [Refer also to page 20 of this report]. 


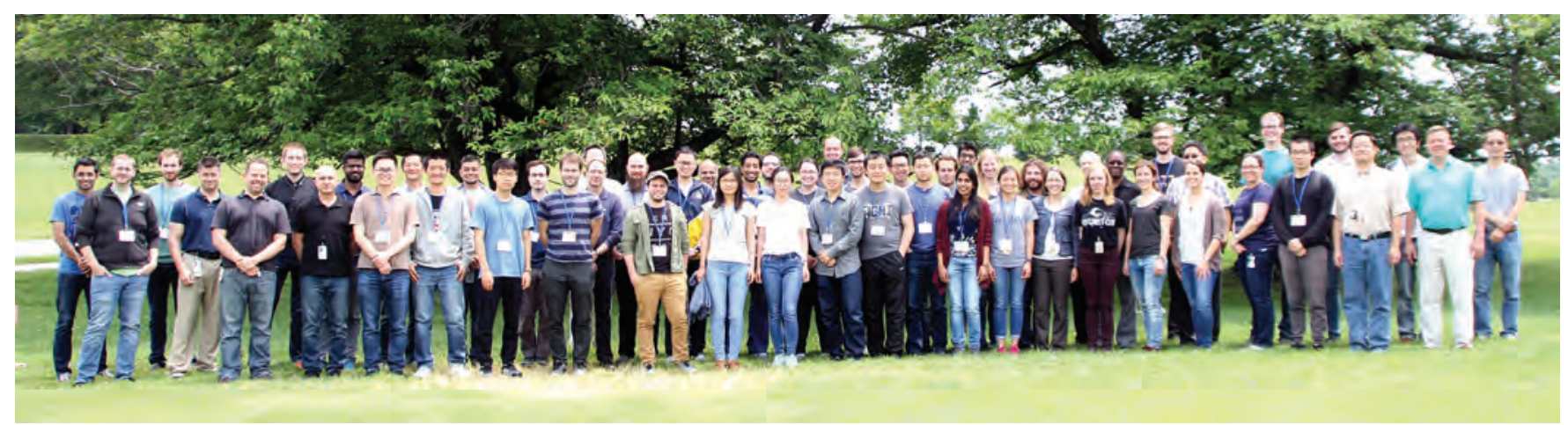

FIGURE 3: Participants and instructors in the 2017 CHRNS Summer School "Methods and Applications of Neutron Spectroscopy."

\section{Education and Outreach}

CHRNS sponsored a variety of educational programs and activities tailored to specific age groups and professions. The twenty-third annual summer school, held on June 19 - June 23, 2017 was entitled "Methods and Applications of Neutron Spectroscopy." Thirty-four graduate and postdoctoral students from 26 universities participated in the school. Guest lecturers were Prof. Kate Ross from Colorado State University, Prof. Eric Bloch the University of Delaware, and Dr. Elizabeth Kelley, a recent CHRNS hire.

In the fall of 2016 Boualem Hammouda, a CHRNS staff member, offered a web-based SANS course. A total of 93 students enrolled for the course, and more than 50 students from 30 universities across the U.S. obtained credit. The students used the free book written by the instructor, and the course included homework. Current plans are to offer this online course again in two years.

Other CHRNS staff scientists participated in teaching graduate courses on scattering theory and techniques at nearby universities including the University of Maryland and University of Delaware. Some of the courses involve lab practicals performed at the NCNR using CHRNS instruments. In the fall of 2017, Yun Liu was the primary instructor for a course entitled "Advanced Characterization of Soft Matter" at the University of Maryland. The course focused on both theories and experimental works of studying structure and dynamics of soft matter materials using scattering techniques (light, X-ray and neutron scattering).

As part of its expanding education and outreach effort, CHRNS offers to university-based research groups with BTAC-approved experimental proposals the opportunity to request travel support for an additional graduate student to participate in the experiment. This support is intended to enable prospective thesis students, for example, to acquire first-hand experience with a technique that they may later use in their own research. Announcements of this program are sent to all of the university groups whose experimental proposals receive beam time from the BTAC. Recipients of the announcement are encouraged to consider graduate students from under-represented groups for this opportunity. The program is also advertised on the NCNR's website at http://www.ncnr.nist.gov/outreach.html.
As in previous years, CHRNS participated in NIST's Summer Undergraduate Research Fellowship (SURF) program. In 2017 CHRNS hosted 15 SURF students, including one previous participant in the SHIP program and one returning SURF intern who had also been in the SHIP program. The students participated in research projects such as $\mathrm{CO}_{2}$ adsorption in metal organic frameworks, structural characterization of insulin under shear, evaluation of a new scintillator detector system for CANDOR, modernization of a tritium air monitoring system, design of a new octo-strain device for sheet metal neutron testing, and development of a web-based service for distributing curve fitting algorithms onto remote server clusters. They presented their work at the NIST SURF colloquium in early August 2017 in sessions moderated by program officers from the National Science Foundation.

CHRNS initiated a Research Experiences for Teachers (RET) program in 2010. For the summer of 2017, the program hosted two teachers from Montgomery County, MD. John Pisanic from Damascus High School studied the effect of various cations on the formation of anionic wormlike micelles using SANS under the guidance of Dr. Katie Weigandt. Benjamin Brooks from Magruder High School investigated the effect of citrate on casein micelles with Dr. Susana Teixeira from the NCNR. Mr. Brooks also highlighted his research in an oral presentation in early August.

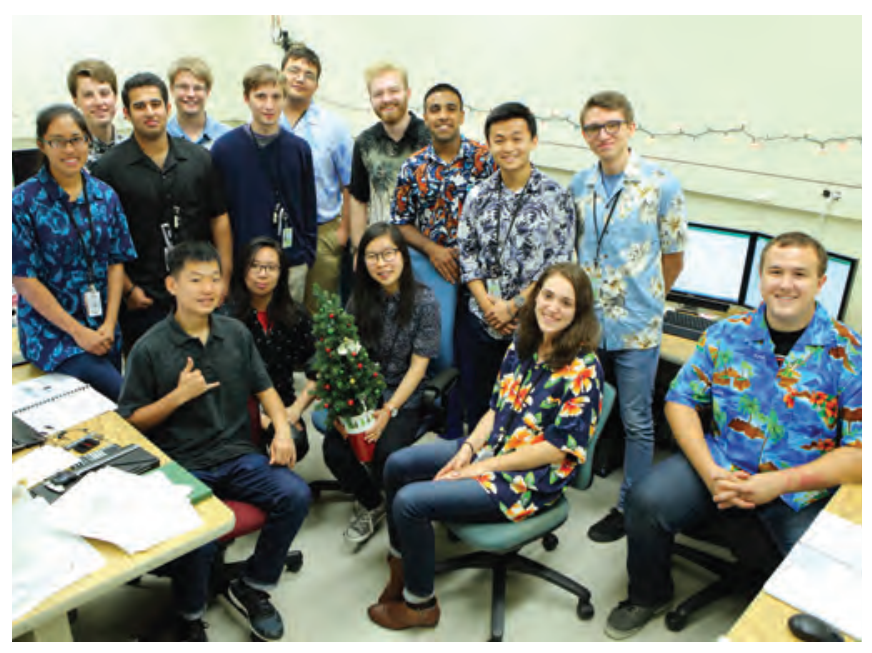

FIGURE 4: The 2017 SURF students. 


\section{Elementary, Middle, and High School Activities}

The Summer High School Intern Program (SHIP) is a very successful, competitive NIST-wide program for students who are interested in performing scientific research during the summer. CHRNS hosted ten interns from local high schools. The students studied the behavior of solid electrolytes for applications in solid state batteries, explored the length scales for magnetic coupling in thin film topological insulators, developed classifications schemes for small angle scattering data using neural networks, and implemented updates to the fume hood monitoring system. The results of the students' summer investigations were highlighted in a NIST-wide poster session, as well as in a well-attended symposium.

The annual Summer Institute for Middle School Science Teachers brings middle school science teachers from across the United States to NIST for two weeks in order to give them a better understanding of the scientific process. Each year, CHRNS hosts the teachers for a one-day introduction to neutron scattering. This year the Summer Institute hosted twenty-four participants from eleven states, including Oregon, Utah, and Colorado. The teachers learned about the types of experiments performed using neutrons and toured the neutron guide hall. To bring home projects suitable for

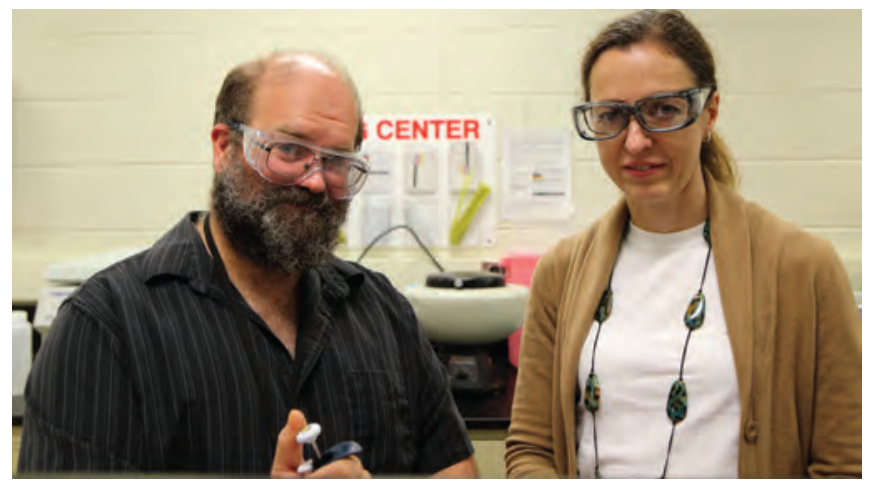

FIGURE 5: Susana Teixeira works with Benjamin Brooks from Magruder High School to prepare samples for an upcoming SANS experiment.

middle school students, they then learned how to grow crystals of "alum" (hydrated aluminum potassium sulfate).

A large number of specialized tours for middle school, high school, and university students were also offered throughout the year. At local schools CHRNS staff members also give science-based talks or lead hands-on demonstrations at local schools, participate in STEM events, and even volunteer as robotics coaches.

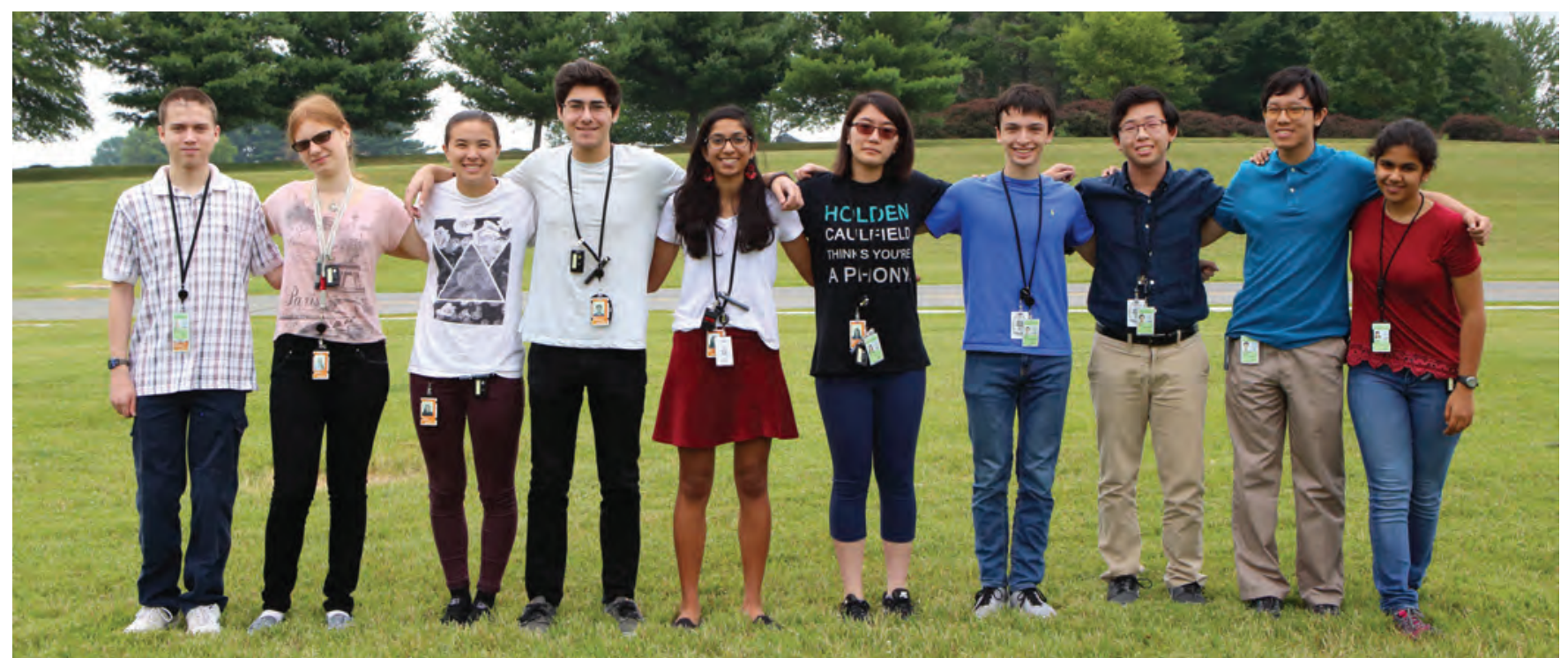

FIGURE 6: The 2017 SHIP interns. 


\section{Awards}
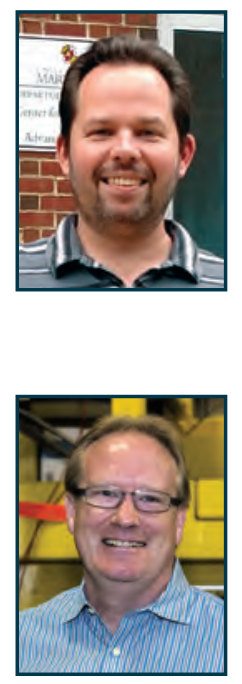

The White House announced that Nick Butch of the NCNR is a recipient of the Presidential Early Career Award for Scientists and Engineers (PECASE). Nick is being honored "for his significant contributions to understanding the interplay of magnetism with superconductivity and revealing observations about superconducting materials."

Craig Brown of the NCNR received the Samuel Wesley Stratton Award "For revealing mechanisms that yield great improvements in gas-separation and catalytic processes in microporous materials." With the Samuel Wesley Stratton Award, NIST honors outstanding scientific or engineering achievements in support of NIST objectives. This is the highest scientific award that NIST bestows.

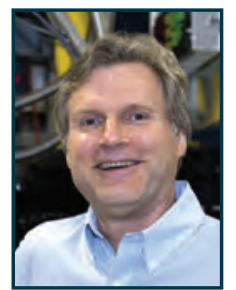

The NCNR's Terry Udovic received the 2016 Department of Commerce Bronze Medal. The citation for Terry's award is "For the discovery of unparalleled superionic conduction in solid electrolyte materials suitable for rechargeable battery applications."

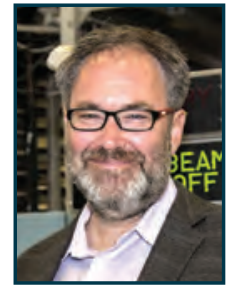

Ron Jones of the Material Measurement Laboratory has been recognized with the George A. Uriano Award for his leadership in creating the nSoft consortium, a new model designed to enhance industrial interest in and use of national user facilities like the NIST Center for Neutron Research. The George A. Uriano Award, is granted for outstanding achievements by NIST staff in building or strengthening NIST extramural programs, with emphasis on fostering U.S. competitiveness and business excellence.

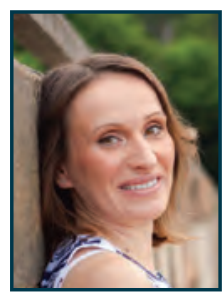

Congratulations to Mirjana Dimitrievska of the NCNR and NREL who received a Most Outstanding Poster award at the Post-doc Poster Presentation sponsored by the NIST chapter of Sigma Xi. Mirjana was recognized in the category of Energy, Water, Environment, and Polymers. Her poster is entitled "Neutron scattering studies of hydrogenous materials for next-generation energy storage."

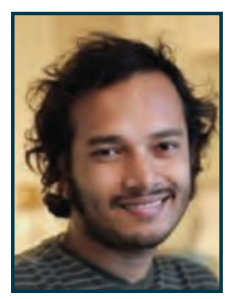

Congratulations also to the NCNR's

Md Taufique Hassan who received an Outstanding Poster award in the area of Physics, Computer Modeling, and Simulation. His poster is entitled "The precision measurement of the electron-antineutrino correlation in neutron beta decay."

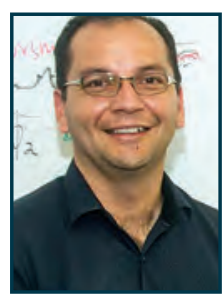

Congratulations to Ramon Castañeda-Priego who is this year's recipient of the Mexican Academy of Science's Award for Young Scientists in the area of "ciencias exactas". Ramon is a professor of physics at the University of Guanajuato who is interested in the theory of soft materials. Ramon is spending his sabbatical here at the NCNR.

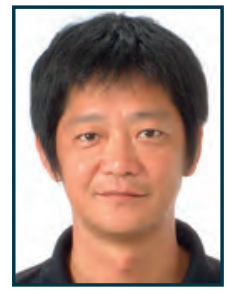

The President of the Japanese Society for Neutron Science has selected a paper written by Michihiro Nagao of the NCNR and Takumi Hawa for the Hamon President's Choice Award. The paper entitled "Thickness fluctuations in surfactant bilayers" was published in Hamon (the Japanese Journal of Neutron Science) in November 2014. Five papers published over the two-year period from November 2014 to August 2016 were selected for this honor.

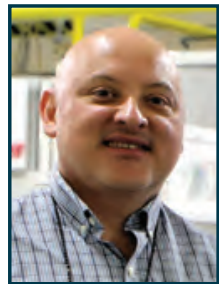

The NCNR's Jose Rodriguez-Rivera has been named "Investigador Nacional - nivel 1" (National Researcher - level 1) by the National Council of Science and Technology of Mexico. Jose is one of 200 researchers in all areas of science and engineering working outside of Mexico to receive this recognition. 

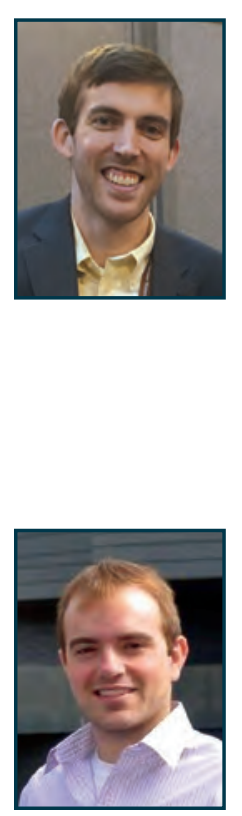

Congratulations to Cameron Shelton who won 1st prize in the DPOLY poster competition at the recent APS March Meeting. His poster was entitled "Quantifying lithium salt distributions in nanostructured ion-conducting polymer domains: A neutron reflectivity study". Cameron was a graduate student in the Epps group at the University of Delaware, and during that time he spent several months stationed at the NCNR working with Joe Dura and Ron Jones.

Congratulations to Justin Milner of the NCNR for winning the High-Quality Poster Award from the Materials Processing and Manufacturing Division at the 2017 meeting of The Minerals, Metals, and Materials Society in San Diego. His poster was titled "Octo-Strain: A Novel Multiaxial Loading Device for In-situ Stress Measurements through Neutron Diffraction."

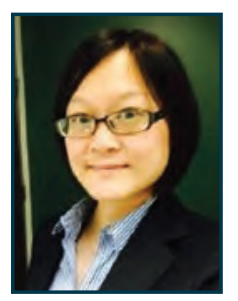

Congratulations to Wei-Shan Chiang of the NCNR, who has been named a Distinguished Speaker by the Society of Petrophysicists and Well Log Analysts (SPWLA) for 20172018. She was selected for her paper entitled "Simultaneous Neutron and X-ray Imaging of 3D Kerogen and Fracture Structure for Flow Path in Shales", which she presented at the 58th Annual SPWLA Symposium in June 2017. As a Distinguished Speaker, she will visit and speak at local chapters of SPWLA throughout the coming year.

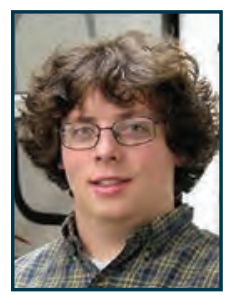

The NCNR's Colin Heikes was awarded the Runner-Up Poster Prize for the 2017 International Workshop on Oxide Electronics held in Chicago in September. His poster title was "Engineering magnetism and polarization domain structures in $\mathrm{BiFeO}_{3}$ containing thin films through superlattice structuring."

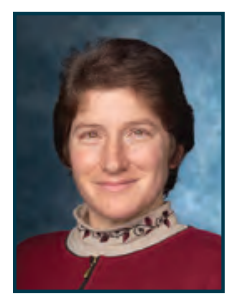

The Society of Rheology has announced that Professor Julia A. Kornfield, Professor of Chemical Engineering at California Institute of Technology is the recipient of the $\mathbf{2 0 1 7}$ Bingham Medal. The award was presented at the 89th SoR Annual Meeting in Denver, Colorado. Professor Kornfield's research includes the understanding, on a molecular level, of a broad range of macroscopic properties of polymers. Prof. Kornfield is a long time NCNR user.

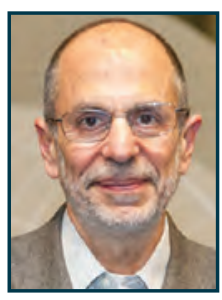

Prof. Robert J. Cava of Princeton University has been awarded the 2016 MRS Medal. The MRS medal is awarded for a specific outstanding recent discovery or advancement that has a major impact on the progress of a materials-related field. Prof. Cava is being honored "for pioneering contributions in the discovery of new classes of 3D Topological Insulators." Prof. Cava has published more than 100 papers containing data from the NCNR.

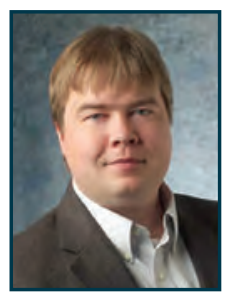

Michael Hore, Assistant Professor in the Department of Macromolecular Science and Engineering at Case Western Reserve University, has been named the UKPPG/ DPOLY lecturer for 2017 by the American Physical Society. The UKPPG/DPOLY Lecture Exchange was established in 2009 recognize research excellence and promise by polymer physicists just beginning their independent careers, and to strengthen ties between DPOLY and the Polymer Physics Group (PPG) of the Institute of Physics. Michael recently completed his post-doc at the NCNR.

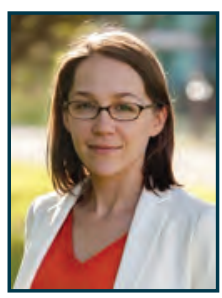

Kate Ross, assistant professor of physics at Colorado State University, was awarded the American Physical Society's 2016 Valley Prize "for the elucidation of quantum frustrated magnetism and its expression in the ground state selection of pyrochlore magnets." The biennial prize recognizes "one individual in the early stages of his or her career for an outstanding scientific contribution to physics that is deemed to have significant potential for a dramatic impact on the field." Kate was a recent post-doc at the NCNR and Johns Hopkins.

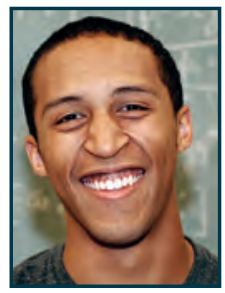

Guy Marcus appeared in Forbes' list of "30 Under 30" in Science for 2016. Guy uses neutron scattering in concert with theory and other experimental techniques, to study the impact of magnetic and electronic structure on quantum materials. According to Forbes, "this field of physics has demonstrated ability to develop new materials with a variety of potential applications, from high-temperature superconductors for transmitting power more efficiently to chemically-tuned magnetic materials that could enable new kinds of computing." Guy is an NSF Graduate Research Fellow studying under Collin L. Broholm at the Institute for Quantum Matter at Johns Hopkins. 


\section{Publications: August 1, 2016 to July 31, 2017}

Adrien, V., Rayan, G., Reffay, M., Porcar, L., Maldonado, A., Ducruix, A., Urbach, W., Taulier, N., "Characterization of a Biomimetic Mesophase Composed of Nonionic Surfactants and an Aqueous Solvent," Langmuir 32(40), 10268 (2016). [CHRNS]

Al-Qasir, I., Qteish, A., "Neutron Filter Efficiency of Beryllium and Magnesium Fluorides," J. Appl. Crystallogr. 50(2), 441 (2017).

Alfeld, M., De Nolf, W., Cagno, S., Appel, K., Siddons, D.P., Kuczewski, A., Janssens, K., Dik, J., Trentelman, K., Walton, M., Satorius, A., "Revealing Hidden Paint Layers in Oil Paintings by Means of Scanning Macro-XRF: A Mock-up Study Based on Rembrandt's 'An Old Man in Military Costume,'" J. Anal. Atom. Spectrom. 28(1), 40 (2013).

Allen, A.J., Zhang, F., Kline, R.J., Guthrie, W.F., Ilavsky, J., "NIST Standard Reference Material 3600: Absolute Intensity Calibration Standard for Small-Angle X-ray Scattering," J. Appl. Crystallogr. 50(2), 462 (2017). [CHRNS]

Alves, L.M.S., Oliveira, F.S., de Lima, B.S., da Luz, M.S., Rebello, A., Masunaga, S.H., Neumeier, J.J., Giles, C., Leão, J.B., dos Santos, C.A.M., "Evidence of Phase Transitions in $\mathrm{MoO}_{2}$ Single Crystals," J. Alloy Compd. 705, 764 (2017).

Ang, J., Ma, D., Lund, R., Keten, S., Xu, T., "Internal Structure of $15 \mathrm{~nm}$ 3-Helix Micelle Revealed by Small-Angle Neutron Scattering and Coarse-Grained MD Simulation," Biomacromolecules 17(10), 3262 (2016). [CHRNS]

Arneson, S., Şahin, D., "Cyber Security using Multi-Threaded Architecture Data Diode at the NBSR," in "ANS Summer," edited by ANS, (2017 ANS Annual Meeting, June 2017, San Francisco, CA), 1 (2017).

Ashenfelter, J., Balantekin, A.B., Band, H.R., Barclay, G., Bass, C.D., Berish, D., Bignell, L., Bowden, N.S., Bowes, A., Brodsky, J.P., Bryan, C.D., Cherwinka, J.J., Chu, R., Classen, T., Commeford, K., Conant, A.J., Davee, D., Dean, D., Deichert, G., Diwan, M.V., Dolinski, M.J., Dolph, J., DuVernois, M., Erickson, A.S., Febbraro, M.T., Gaison, J.K., Galindo-Uribarri, A., Gilje, K., Glenn, A., Goddard, B.W., Green, M., Hackett, B.T., Han, K., Hans, S., Heeger, K.M., Heffron, B., Insler, J., Jaffe, D.E., Jones, D., Langford, T.J., Littlejohn, B.R., Martinez Caicedo, D.A.,
Matta, J.T., McKeown, R.D., Mendenhall, M.P., Mueller, P.E., Mumm, H.P., Napolitano, J., Neilson, R., Nikkel, J.A., Norcini, D., Pushin, D., Qian, X., Romero, E., Rosero, R., Seilhan, B.S., Sharma, R., Sheets, S., Surukuchi, P.T., Trinh, C., Varner, R.L., Viren, B., Wang, W., White, B., White, C., Wilhelmi, J., Williams, C., Wise, T., Yao, H., Yeh, M., Yen, Y.-R., Zangakis, G.Z., Zhang, C., Zhang, X., "The PROSPECT Physics Program," J. Phys. G Nucl. Partic. 43(11), 113001 (2016).

Ashkar, R., Hore, M.J.A., Ye, X., Natarajan, B., Greybush, N.J., Lam, T., Kagan, C.R., Murray, C.B., "Rapid Large-Scale Assembly and Pattern Transfer of One-Dimensional Gold Nanorod Superstructures," ACS Appl. Mater. Interfaces 9, 25513 (2017). [CHRNS]

Bahadur, J., Medina, C.R., He, L., Melnichenko, Y.B., Rupp, J.A., Blach, T.P., Mildner, D.F.R., "Determination of Closed Porosity in Rocks by Small-Angle Neutron Scattering," J. Appl. Crystallogr. 49(6), 2021 (2016). [CHRNS]

Balz, C., Lake, B., Reuther, J., Luetkens, H., Schönemann, R., Herrmannsdörfer, T., Singh, Y., Nazmul Islam, A.T.M., Wheeler, E.M., Rodriguez-Rivera, J.A., Guidi, T., Simeoni, G.G., Baines, C., Ryll, H., "Physical Realization of a Quantum Spin Liquid Based on a Complex Frustration Mechanism," Nat. Phys. 12(10), 942 (2016). [CHRNS]

Balz, C., Lake, B., Nazmul Islam, A.T.M., Singh, Y., Rodriguez-Rivera, J.A., Guidi, T., Wheeler, E.M., Simeoni, G.G., Ryll, H., "Magnetic Hamiltonian and Phase Diagram of the Quantum Spin Liquid $\mathrm{Ca}_{10} \mathrm{Cr}_{7} \mathrm{O}_{28}$," Phys. Rev. B 95(17), 174414 (2017). [CHRNS]

Beckingham, L.E., Steefel, C.I., Swift, A.M., Voltolini, M., Yang, L., Anovitz, L.M., Sheets, J.M., Cole, D.R., Kneafsey, T.J., Mitnick, E.H., Zhang, S., Landrot, G., Ajo-Franklin, J.B., DePaolo, D.J., Mito, S., Xue, Z., "Evaluation of Accessible Mineral Surface Areas for Improved Prediction of Mineral Reaction Rates in Porous Media," Geochim. Cosmochim. Ac. 205, 31 (2017). [CHRNS]

Bellur, K., Konduru, V., Medici, E.F., Hussey, D.S., Jacobson, D.L., LaManna, J.M., Allen, J.S., Choi, C.K., "Visualization of the Evaporation and Condensation Phenomena in Cryogenic Propellants," J. Flow Vis. Image Proc. 23(1-2), 137 (2016). 
Benedetto, A., Kearley, G.J., "Elastic Scattering Spectroscopy (ESS): An Instrument-Concept for Dynamics of Complex (Bio-) Systems from Elastic Neutron Scattering," Sci. Rep. 6, 34266 (2016). [CHRNS]

Bergeron, D.E., Mumm, H.P., Tyra, M.A., "Phase Stability and Lithium Loading Capacity in a Liquid Scintillation Cocktail," J. Radioanal. Nucl. Ch., in press.

Bertrand, C.E., Self, J.L., Copley, J.R.D., Faraone, A., "Nanoscopic Length Scale Dependence of Hydrogen Bonded Molecular Associates' Dynamics in Methanol," J. Chem. Phys. 146(19), 194501 (2017). [CHRNS]

Bethea, K., Fernandez-Baca, J., Lynn, J., "In Memoriam: Herbert A. Mook (1939-2016)," Neutron News 28(2), 21 (2017).

Bhattacharya, P., Ramasamy, U.S., Krueger, S., Robinson, J.W., Tarasevich, B.J., Martini, A., Cosimbescu, L., "Trends in Thermoresponsive Behavior of Lipophilic Polymers," Ind. Eng. Chem. Res. 55(51), 12983 (2016).

Bradbury, R., Nagao, M., "Effect of Charge on the Mechanical Properties of Surfactant Bilayers," Soft Matter 12(46), 9383 (2016). [CHRNS]

Brambleby, J., Manson, J.L., Goddard, P.A., Stone, M.B., Johnson, R.D., Manuel, P., Villa, J.A., Brown, C.M., Lu, H., Chikara, S., Zapf, V., Lapidus, S.H., Scatena, R., Macchi, P., Chen, Y.-S., Wu, L.-C., Singleton, J., "Combining Microscopic and Macroscopic Probes to Untangle the Single-Ion Anisotropy and Exchange Energies in an $S=1$ Quantum Antiferromagnet," Phys. Rev. B 95(13), 134435 (2017).

Brok, E., Frandsen, C., Lefmann, K., McEnroe, S., Robinson, P., Burton, B.P., Hansen, T.C., Harrison, R., "Spin Orientation in Solid Solution Hematite-ilmenite," Am. Mineral. 102(6), 1234 (2017).

Bryan, M.S., Prisk, T.R., Sherline, T.E., Diallo, S.O., Sokol, P.E., "Bulklike Excitations in Nanoconfined Liquid Helium," Phys. Rev. B 95(14), 144509 (2017).

Bryan, M.S., Prisk, T.R., Azuah, R.T., Stirling, W.G., Sokol, P.E., "The Momentum Distribution of Liquid ${ }^{3} \mathrm{He}$," Europhys. Lett. 115(6), 66001 (2016).

Bukovsky, E.V., Peryshkov, D.V., Wu, H., Zhou, W., Tang, W.S., Jones, W.M., Stavila, V., Udovic, T.J., Strauss, S.H., "Comparison of the Coordination of $\mathrm{B}_{12} \mathrm{~F}_{12}{ }^{2-}, \mathrm{B}_{12} \mathrm{Cl}_{12}{ }^{2-}$, and $\mathrm{B}_{12} \mathrm{H}_{12}{ }^{2-}$ to $\mathrm{Na}^{+}$zin the Solid State: Crystal Structures and Thermal Behavior of $\mathrm{Na}_{2}\left(\mathrm{~B}_{12} \mathrm{~F}_{12}\right), \mathrm{Na}_{2}\left(\mathrm{H}_{2} \mathrm{O}\right)_{4}\left(\mathrm{~B}_{12} \mathrm{~F}_{12}\right), \mathrm{Na}_{2}\left(\mathrm{~B}_{12} \mathrm{Cl} \mathrm{l}_{12}\right)$, and $\mathrm{Na}_{2}\left(\mathrm{H}_{2} \mathrm{O}\right)_{6}\left(\mathrm{~B}_{12} \mathrm{Cl}_{12}\right)$," Inorg. Chem. 56(8), 4369 (2017).
Butch, N.P., Ran, S., Jeon, I., Kanchanavatee, N., Huang, K., Breindel, A., Maple, M.B., Stillwell, R.L., Zhao, Y., Harriger, L., Lynn, J.W., "Distinct Magnetic Spectra in the Hidden Order and Antiferromagnetic Phases in $\mathrm{URu}_{2-x} \mathrm{Fe}_{x} \mathrm{Si}_{2}$," Phys. Rev. B 94(20), 201102 (2016).

Calabrese, M.A., Wagner, N.J., "New Insights from Rheo-SANS," in "Wormlike Micelles: Advances in Systems, Characterisation and Applications," edited by Dreiss, C.A. and Feng, Y., (Thomas Graham House, Cambridge, United Kingdom) Chap. 8, 193 (2017).

Calabrese, M.A., Rogers, S.A., Porcar, L., Wagner, N.J., "Understanding Steady and Dynamic Shear Banding in a Model Wormlike Micellar Solution," J. Rheol. 60(5), 1001 (2016).

Cao, H., Guo, J., Chang, F., Pistidda, C., Zhou, W., Zhang, X., Santoru, A., Wu, H., Schell, N., Niewa, R., Chen, P., Klassen, T., Dornheim, M., "Transition and Alkali Metal Complex Ternary Amides for Ammonia Synthesis and Decomposition," Chem.-Eur. J. 23(41), 9766 (2017).

Carlson, S.B., Blackman, M.J., Bishop, R.L., "Texas Mission Ceramics: Origins of Manufacture and Distribution During the Eighteenth Century," Hist. Archaeol. 50(4), 65 (2016).

Castellanos, M.M., McAuley, A., Curtis, J.E., "Investigating Structure and Dynamics of Proteins in Amorphous Phases using Neutron Scattering," Comput. Struct. Biotechnol. J. 15, 117 (2017). [CHRNS]

Castellanos, M.M., Clark, N.J., Watson, M.C., Krueger, S., McAuley, A., Curtis, J.E., "Role of Molecular Flexibility and Colloidal Descriptions of Proteins in Crowded Environments from Small-Angle Scattering," J. Phys. Chem. B 120(49), 12511 (2016). [CHRNS]

Chen, J., Wang, X., Kline, S.R., Liu, Y., "Size Effects of Solvent Molecules on the Phase Behavior and Effective Interaction of Colloidal Systems with the Bridging Attraction," J. Phys.: Condens. Matter 28, 455102 (2016).

Chen, J., Gao, Q., Sanson, A., Jiang, X., Huang, Q., Carnera, A., Rodriguez, C.G., Olivi, L., Wang, L., Hu, L., Lin, K., Ren, Y., Lin, Z., Wang, C., Gu, L., Deng, J., Attfield, J.P., Xing, X., "Tunable Thermal Expansion in Framework Materials through Redox Intercalation," Nat. Commun. 8, 14441 (2017).

Chen, M., Zhao, E., Chen, D., Wu, M., Han, S., Huang, Q., Yang, L., Xiao, X., Hu, Z., "Decreasing Li/Ni Disorder and Improving the Electrochemical Performances of Ni-Rich $\mathrm{LiNi}_{0.8} \mathrm{CO}_{0.1} \mathrm{Mn}_{0.1} \mathrm{O}_{2}$ by Ca Doping," Inorg. Chem. 56(14), 8355 (2017). 
Chen, T., Foley, B.J., Park, C., Brown, C.M., Harriger, L.W., Lee, J., Ruff, J., Yoon, M., Choi, J.J., Lee, S.-H., "Entropy-Driven Structural Transition and Kinetic Trapping in Formamidinium Lead lodide Perovskite," Sci. Adv. 2(10), e1601650 (2016).

Chen, T., Chen, W.-L., Foley, B.J., Lee, J., Ruff. J.P.C., Ko, J.Y.P., Brown, C.M., Harriger, L.W., Zhang, D., Park, C., Yoon, M., Chang, Y.-M., Choi, J.J., Lee, S.-H., "Origin of Long Lifetime of Band-Edge Charge Carriers in Organic-Inorganic Lead lodide Perovskites," P. Natl. A. Sci. USA 114(29), 7519 (2017).

Chen, W.C., Barker, J.G., Jones, R., Krycka, K.L., Watson, S.M., Gagnon, C., Perevozchivoka, T., Butler, P., Gentile, T.R., "Spin-Analyzed SANS for Soft Matter Applications," J. Phys. Conf. Ser. 862(1), 012004 (2017). [CHRNS]

Chen, W.C., Gentile, T.R., Ye, Q., Kirchhoff, A., Watson, S.M., Rodriguez-Rivera, J.A., Qiu, Y., Broholm, C., "Recent Advancements of Wide-Angle Polarization Analysis with ${ }^{3} \mathrm{He}$ Neutron Spin Filters," J. Phys. Conf. Ser. 746(1), 012016 (2016). [CHRNS]

Chen-Mayer, H.H., Fuld, M.K., Hoppel, B., Judy, P.F., Sieren, J.P., Guo, J., Lynch, D.A., Possolo, A., Fain, S.B., "Standardizing CT Lung Density Measure Across Scanner Manufacturers," Med. Phys. 44(3), 974 (2017).

Chiang, W.-S., LaManna, J.M., Hussey, D.S., Jacobson, D.L., Liu, Y., Zhang, J., Georgi, D.T., Chen, J.-H., "Simultaneous Neutron and X-ray Imaging of 3D Kerogen and Fracture Structure in Shales," in "SPWLA 58th Annual Logging Symposium," edited by Society of Petrophysicists and Well-Log Analysts, (SPWLA 58th Annual Logging Symposium, May 2017, Oklahoma City, Oklahoma), 1 (2017).

Chiang, W.-S., Fratini, E., Baglioni, P., Chen, J.-H., Liu, Y., "Pore Size Effect on Methane Adsorption in Mesoporous Silica Materials Studied by Small-Angle Neutron Scattering," Langmuir 32(35), 8849 (2016). [CHRNS]

Choi, K.-I., Kim, T.-H., Yuan, G., Satija, S.K., Koo, J., "Dynamics of Entangled Polymers Confined between Graphene Oxide Sheets as Studied by Neutron Reflectivity," ACS Macro Lett., in press.

Cicerone, M.T., Tyagi, M., "Metabasin Transitions are Johari-Goldstein Relaxation Events," J. Chem. Phys. 146(5), 054502 (2017). [CHRNS]

Clarkson, C.R., Solano, N.A., "Combined use of Neutron-Scattering, Fluid-Invasion, and Image-Analysis Techniques to Assess Pore Structure, Accessibility, and Connectivity in Tight Rock," in "The Clay Minerals Society Workshop Lectures Series, Vol. 21," (The Clay Mineral Society, Chantilly, VA) Chap. 2, 15 (2016). [CHRNS]
Collett, B., Bateman, F., Bauder, W.K., Byrne, J., Byron, W.A., Chen, W., Darius, G., DeAngelis, C., Dewey, M.S., Gentile, T.R., Hassan, M.T., Jones, G.L., Komives, A., Laptev, A., Mendenhall, M.P., Nico, J.S., Noid, G., Park, H., Stephenson, E.J., Stern, I., Stockton, K.J.S., Trull, C., Wietfeldt, F.E., Yerozolimsky, B.G., "aCORN: An Experiment to Measure the Electron-Antineutrino Correlation Coefficient in Free Neutron Decay," Rev. Sci. Instrum., in press.

Cooksey, T.J., Singh, A., Le, K.M., Wang, S., Kelley, E.G., He, L., Kesava, S.V., Gomez, E.D., Kidd, B.E., Madsen, L.A., Robertson, M.L., "Tuning Biocompatible Block Copolymer Micelles by Varying Solvent Composition: Core/Corona Structure and Solvent Uptake," Macromolecules 50, 4322 (2017).

Cooper, N.J., Santamaria, A.D., Becton, M.K., Park, J.W., "Neutron Radiography Measurements of In-Situ PEMFC Liquid Water Saturation in 2D \& 3D Morphology Gas Diffusion Layers," Int. J. Hydrogen Energ. 42(25), 16269 (2017).

Cudjoe, E., Hunsen, M., Xue, Z., Way, A.E., Barrios, E., Olson, R.A., Hore, M.J.A., Rowan, S.J., "Miscanthus Giganteus: A Commercially Viable Sustainable Source of Cellulose Nanocrystals," Carbohyd. Polym. 155, 230 (2017). [CHRNS]

Cudjoe, E., Khani, S., Way, A.E., Hore, M.J.A., Maia, J., Rowan, S.J., "Biomimetic Reversible Heat-Stiffening Polymer Nanocomposites," ACS Central Science, in press. [CHRNS]

Cui, X., Yang, Q., Yang, L., Krishna, R., Zhang, Z., Bao, Z., Wu, H., Ren, Q., Zhou, W., Chen, B., Xing, H., "Ultrahigh and Selective $\mathrm{SO}_{2}$ Uptake in Inorganic Anion-Pillared Hybrid Porous Materials," Adv. Mater. 29(28), 1606929 (2017).

Dahal, A., Gunasekera, J., Harriger, L., Lee, S.H., Hor, Y.S., Singh, D.J., Singh, D.K., "Intermediate Regime between Metal and Superconductor Below T=100 K in NiSi," Phys. Rev. B 94(18), 184516 (2016).

Dally, R., Clément, R.J., Chisnell, R., Taylor, S., Butala, M., DoanNguyen, V., Balasubramanian, M., Lynn, J.W., Grey, C.P., Wilson, S.D., "Floating Zone Growth of $\alpha-\mathrm{Na}_{0.90} \mathrm{MnO}_{2}$ Single Crystals," J. Cryst. Growth 459, 203 (2017).

Danks, A.E., Hollamby, M.J., Hammouda, B., Fletcher, D.C., JohnstonBanks, F., Rogers, S.E., Schnepp, Z., "Mechanistic Insights into the Formation of Porous Carbons from Gelatin," J. Mater. Chem. A 5(23), 11644 (2017).

Dao, M.M., Domach, M.M., Walker, L.M., "Impact of Dispersed Particles on the Structure and Shear Alignment of Block Copolymer Soft Solids," J. Rheol. 61(2), 237 (2017). [CHRNS] 
Darius, G., Byron, W.A., DeAngelis, C.R., Hassan, M.T., Wietfeldt, F.E., Collett, B., Jones, G.L., Dewey, M.S., Mendenhall, M.P., Nico, J.S., Park, H., Komives, A., Stephenson, E.J., "Measurement of the Electron-Antineutrino Angular Correlation in Neutron $\beta$ Decay," Phys. Rev. Lett. 119(4), 042502 (2017).

de Beer, F., van der Merwe, J.-H., Bessarabov, D., "PEM Water Electrolysis: Preliminary Investigations using Neutron Radiography," Phys. Procedia 88, 19 (2017).

de Haan, V.-O., Gommers, R., Rowe, J.M., "Thermodynamic Calculations of a Two-Phase Thermosyphon Loop for Cold Neutron Sources," Cryogenics 85, 30 (2017).

Dehvari, K., Lin, K.-S., Hammouda, B., "Small-Angle Neutron Scattering Studies of Microenvironmental and Structural Changes of Pluronic Micelles upon Encapsulation of Paclitaxel," J. Taiwan Inst. Chem. E 71, 405 (2017). [CHRNS]

Diallo, S.O., Lin, J.Y.Y., Abernathy, D.L., Azuah, R.T., "Momentum and Energy Dependent Resolution Function of the ARCS Neutron Chopper Spectrometer at High Momentum Transfer: Comparing Simulation and Experiment," Nucl. Instrum. Meth. A 835, 34 (2016)

Dimeo, R., "Sketchnoting: An Analog Skill in the Digital Age," ACM Computers \& Society 46(3), 9 (2016).

Dimitrievska, M., White, J.L., Zhou, W., Stavila, V., Klebanoff, L.E., Udovic, T.J., "Structure-Dependent Vibrational Dynamics of $\mathrm{Mg}\left(\mathrm{BH}_{4}\right)_{2}$ Polymorphs Probed with Neutron Vibrational Spectroscopy and First-Principles Calculations," Phys. Chem. Chem. Phys. 18(36), 25546 (2016).

Dimitrievska, M., Boero, F., Litvinchuk, A.P., Delsante, S., Borzone, G., Pérez-Rodríguez, A., Izquierdo-Roca, V., "Structural Polymorphism in 'Kesterite' $\mathrm{Cu}_{2} \mathrm{ZnSnS}_{4}$ : Raman Spectroscopy and First-Principles Calculations Analysis," Inorg. Chem. 56(6), 3467 (2017).

Dimitrievska, M., Ivetić, T.B., Litvinchuk, A.P., Fairbrother, A., Miljević, B.B., Štrbac, G.R., Pérez-Rodríguez, A., LukićPetrović, S.R., "Eu3+-Doped Wide Band Gap $\mathrm{Zn}_{2} \mathrm{SnO}_{4}$ Semiconductor Nanoparticles: Structure and Luminescence," J. Phys. Chem. C 120(33), 18887 (2016).

Du, P., Li, A., Li, X., Zhang, Y., Do, C., He, L., Rick, S.W., John, V.T., Kumar, R., Zhang, D., "Aggregation of Cyclic Polypeptoids Bearing Zwitterionic End-Groups with Attractive Dipole-Dipole and Solvophobic Interactions: A Study by Small-Angle Neutron Scattering and Molecular Dynamics Simulation," Phys. Chem. Chem. Phys. 19(22), 14388 (2017). [CHRNS]
Duncan, T.T., Berrie, B.H., Weiss, R.G., "Colloidal Properties of Aqueous Poly(Vinyl Acetate)-Borate Dispersions with ShortChain Glycol Ethers," Chem. Phys. Chem. 17(16), 2535 (2016).

Dura, J.A., Rus, E.D., Kienzle, P.A., Maranville, B.B., "Nanolayer Analysis by Neutron Reflectometry," in "Nanolayer Research: Methodology and Technology for Green Chemistry," edited by Toyoko, I., (Elsevier-Science and Technology Books, Amsterdam) Chap. 5, 155 (2017).

Eberle, A.P.R., King, H.E., Ravikovitch, P.I., Walters, C.C., Rother, G., Wesolowski, D.J., "Direct Measure of the Dense Methane Phase in Gas Shale Organic Porosity by Neutron Scattering," Energ. Fuel. 30(11), 9022 (2016).

Eells, R., Barros, M., Scott, K.M., Karageorgos, I., Heinrich, F., Lösche, M., "Structural Characterization of Membrane-Bound Human Immunodeficiency Virus-1 Gag Matrix with Neutron Reflectometry," Biointerphases 12(2), 02D408 (2017).

Fallarino, L., Kirby, B.J., Pancaldi, M., Riego, P., Balk, A.L., Miller, C.W., Vavassori, P., Berger, A., "Magnetic Properties of Epitaxial $\mathrm{CoCr}$ Films with Depth-Dependent Exchange-Coupling Profiles," Phys. Rev. B 95(13), 134445 (2017).

Flanagan, T.B., Wang, D., Luo, S., "Thermodynamics of Hydrogen Absorption (Desorption) in Unoxidized and Internally Oxidized Pd-Ti Alloys," J. Alloy Compd. 701, 981 (2017).

Fobes, D.M., Zaliznyak, I.A., Tranquada, J.M., Xu, Z., Gu, G., He, X.-G., Ku, W., Zhao, Y., Matsuda, M., Ovidiu Garlea, V., Winn, B., "Forbidden Phonon: Dynamical Signature of Bond Symmetry Breaking in the Iron Chalcogenides," Phys. Rev. B 94(12), 121103 (2016).

Frampton, M.K., Crocker, J., Gilbert, D.A., Curro, N., Liu, K., Schneeloch, J.A., Gu, G.D., Zieve, R.J., "First-Order Reversal Curve of the Magnetostructural Phase Transition in FeTe," Phys. Rev. B 95(21), 214402 (2017).

Gao, J., Mwasame, P.M., Wagner, N.J., "Thermal Rheology and Microstructure of Shear Thickening Suspensions of Silica Nanoparticles Dispersed in the Ionic Liquid $\left[\mathrm{C}_{4} \mathrm{mim}\right]\left[\mathrm{BF}_{4}\right]_{\text {," }}$ J. Rheol. 61(3), 525 (2017). [CHRNS]

Gao, Q., Chen, J., Sun, Q., Chang, D., Huang, Q., Wu, H., Sanson, A., Milazzo, R., Zhu, H., Li, Q., Liu, Z., Deng, J., Xing, X., "Switching between Giant Positive and Negative Thermal Expansions of a $\mathrm{YFe}(\mathrm{CN})_{6}$-Based Prussian Blue Analogue Induced by Guest Species," Angew. Chem. Int. Edit. 56(31), 9023 (2017). 
Gao, T.R., Fang, L., Fackler, S., Maruyama, S., Zhang, X.H., Wang, L.L., Rana, T., Manchanda, P., Kashyap, A., Janicka, K., Wysocki, A.L., N'Diaye, A.T., Arenholz, E., Borchers, J.A., Kirby, B.J., Maranville, B.B., Sun, K.W., Kramer, M.J., Antropov, V.P., Johnson, D.D., Skomski, R., Cui, J., Takeuchi, I., "Large Energy Product Enhancement in Perpendicularly Coupled MnBi/CoFe Magnetic Bilayers," Phys. Rev. B 94(6), 060411 (2016).

Gao, T., Zhang, X., Ratcliff, II, W., Maruyama, S., Murakami, M., Varatharajan, A., Yamani, Z., Chen, P., Wang, K., Zhang, H., Shull, R., Bendersky, L.A., Unguris, J., Ramesh, R., Takeuchi, I., "Electric-Field Induced Reversible Switching of the Magnetic Easy Axis in $\mathrm{Co} \mathrm{BiFeO}_{3}$ on $\mathrm{SrTiO}_{3}$," Nano Lett. 17(5), 2825 (2017).

Gao, Y., Nieuwendaal, R., Dimitriadis, E.K., Hammouda, B., Douglas, J.F., Xu, B., Horkay, F., "Supramolecular Self-Assembly of a Model Hydrogelator: Characterization of Fiber Formation and Morphology," Gels 2(4), 27 (2016). [CHRNS]

Gaudet, J., Hallas, A.M., Thibault, J., Butch, N.P., Dabkowska, H.A., Gaulin, B.D., "Experimental Evidence for Field-Induced Emergent Clock Anisotropies in the XY Pyrochlore $\mathrm{Er}_{2} \mathrm{Ti}_{2} \mathrm{O}_{7}$," Phys. Rev. B 95(5), 054407 (2017).

Gaudet, J., Hallas, A.M., Maharaj, D.D., Buhariwalla, C.R.C., Kermarrec, E., Butch, N.P., Munsie, T.J.S., Dabkowska, H.A., Luke, G.M., Gaulin, B.D., "Magnetic Dilution and Domain Selection in the $X Y$ Pyrochlore Antiferromagnet $\mathrm{Er}_{2} \mathrm{Ti}_{2} \mathrm{O}_{7}$," Phys. Rev. B 94(6), 060407 (2016).

Gehring, P.M., Parshall, D.E., Harriger, L.W., Stock, C., Xu, G., Li, X., Luo, H., "Phantom Phonon Localization in Relaxors," Nat. Commun., in press.

Germann, N., Gurnon, A.K., Zhou, L., Cook, L.P., Beris, A.N., Wagner, N.J., "Validation of Constitutive Modeling of Shear Banding, Threadlike Wormlike Micellar Fluids," J. Rheol. 60(5), 983 (2016).

Ghazal, A., Lafleur, J.P., Mortensen, K., Kutter, J.P., Arleth, L., Jensen, G.V., "Recent Advances in X-ray Compatible Microfluidics for Applications in Soft Materials and Life Sciences," Lab Chip 16(22), 4263 (2016).

Gilbert, D.A., Liao, J.-W., Kirby, B.J., Winklhofer, M., Lai, C.-H., Liu, K., "Magnetic Yoking and Tunable Interactions in FePt-Based Hard/ Soft Bilayers," Sci. Rep. 6, 32842 (2016).

Gitgeatpong, G., Suewattana, M., Zhang, S., Miyake, A., Tokunaga, M., Chanlert, P., Kurita, N., Tanaka, H., Sato, T.J., Zhao, Y., Matan, K., "High-Field Magnetization and Magnetic Phase Diagram of $\alpha-\mathrm{Cu}_{2} \mathrm{~V}_{2} \mathrm{O}_{7}$," Phys. Rev. B 95(24), 245119 (2017).
Gitgeatpong, G., Zhao, Y., Piyawongwatthana, P., Qiu, Y., Harriger, L.W., Butch, N.P., Sato, T.J., Matan, K., "Nonreciprocal Magnons and Symmetry-Breaking in the Noncentrosymmetric Antiferromagnet," Phys. Rev. Lett. 119(4), 047201 (2017). [CHRNS]

Gómez-Gualdrón, D.A., Wang, T.C., García-Holley, P., Sawelewa, R.M., Argueta, E., Snurr, R.Q., Hupp, J.T., Yildirim, T., Farha, O.K., "Understanding Volumetric and Gravimetric Hydrogen Adsorption Trade-off in Metal-Organic Frameworks," ACS Appl. Mater. Interfaces, in press.

Gómez-Gualdrón, D.A., Colón, Y.J., Zhang, X., Wang, T.C., Chen, Y.-S., Hupp, J.T., Yildirim, T., Farha, O.K., Zhang, J., Snurr, R.Q., "Evaluating Topologically Diverse Metal-Organic Frameworks for Cryo-Adsorbed Hydrogen Storage," Energy Environ. Sci. 9(10), 3279 (2016).

Gordon, M.B., Kloxin, C.J., Wagner, N.J., "The Rheology and Microstructure of an Aging Thermoreversible Colloidal Gel," J. Rheol. 61(1), 23 (2017).

Goto, Y., Tassel, C., Noda, Y., Hernandez, O., Pickard, C.J., Green, M.A., Sakaebe, H., Taguchi, N., Uchimoto, Y., Kobayashi, Y., Kageyama, H., "Pressure-Stabilized Cubic

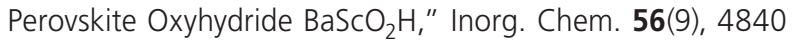
(2017).

Grutter, A.J., Krycka, K.L., Tartakovskaya, E.V., Borchers, J.A., Madhukar Reddy, K.S., Ortega, E., Ponce, A., Stadler, B.J.H., "Complex Three-Dimensional Magnetic Ordering in Segmented Nanowire Arrays," ACS Nano, in press. [CHRNS]

Grutter, A.J., Vailionis, A., Borchers, J.A., Kirby, B.J., Flint, C.L., He, C., Arenholz, E., Suzuki, Y., "Interfacial Symmetry Control of Emergent Ferromagnetism at the Nanoscale," Nano Lett. 16(9), 5647 (2016).

Gu, Y., Kawamoto, K., Zhong, M., Chen, M., Hore, M.J.A., Jordan, A.M., Korley, L.T.J., Olsen, B.D., Johnson, J.A., "Semibatch Monomer Addition as a General Method to Tune and Enhance the Mechanics of Polymer Networks via LoopDefect Control," P. Natl. A. Sci. USA 114(19), 4875 (2017).

Habib, S.A., Khan, A.S., Gnäupel-Herold, T., Lloyd, J.T., Schoenfeld, S.E., "Anisotropy, Tension-Compression Asymmetry and Texture Evolution of a Rare-Earth-Containing Magnesium Alloy Sheet, ZEK100, at Different Strain Rates and Temperatures: Experiments and Modeling," Int. J. Plasticity, in press. 
Hallsteinsen, I., Moreau, M., Grutter, A., Nord, M., Vullum, P.-E., Gilbert, D.A., Bolstad, T., Grepstad, J.K., Holmestad, R., Selbach, S.M., N'Diaye, A.T., Kirby, B.J., Arenholz, E., Tybell, T., "Concurrent Magnetic and Structural Reconstructions at the Interface of (111)-Oriented $\mathrm{La}_{0.7} \mathrm{Sr}_{0.3} \mathrm{MnO}_{3} / \mathrm{LaFeO}_{3}$," Phys. Rev. B 94(20), 201115 (2016).

Halperin, C.T., Bishop, R.L., "Chemical Analysis of Late Classic Maya Polychrome Pottery Paints and Pastes from Central Petén, Guatemala," J. Archaeol. Sci. 69, 118 (2016).

Han, T.-H., Norman, M.R., Wen, J.-J., Rodriguez-Rivera, J.A., Helton, J.S., Broholm, C., Lee, Y.S., "Correlated Impurities and Intrinsic Spin-Liquid Physics in the Kagome Material Herbertsmithite," Phys. Rev. B 94(6), 060409 (2016). [CHRNS]

Han, X., Gong, Y., Fu, K., He, X., Hitz, G.T., Dai, J., Pearse, A., Liu, B., Wang, H., Rubloff, G., Mo, Y., Thangadurai, V., Wachsman, E.D., $\mathrm{Hu}$, L., "Negating Interfacial Impedance in Garnet-Based SolidState Li Metal Batteries," Nat. Mater. 16(5), 572 (2017).

Harriger, L., Disseler, S.M., Gunasekera, J., Rodriguez-Rivera, J., Pixley, J., Manfrinetti, P., Dhar, S.K., Singh, D.K., "Gapped Excitation in Dense Kondo Lattice CePtZn," Phys. Rev. B 95(4), 041102 (2017). [CHRNS]

Hassan, M.T., Bateman, F., Collett, B., Darius, G., DeAngelis, C., Dewey, M.S., Jones, G.L., Komives, A., Laptev, A., Mendenhall, M.P., Nico, J.S., Noid, G., Stephenson, E.J., Stern, I., Trull, C., Wietfeldt, F.E., "The aCORN Backscatter-Suppressed Beta Spectrometer," Nucl. Instrum. Meth. A, in press.

He, Q., Wang, S.-F., Hu, R., Akgun, B., Tormey, C., Peri, S., Wu, D.T., Foster, M.D., "Evidence and Limits of Universal Topological Surface Segregation of Cyclic Polymers," Phys. Rev. Lett. 118(16), 167801 (2017).

He, Q.L., Kou, X., Grutter, A.J., Yin, G., Pan, L., Che, X., Liu, Y., Nie, T., Zhang, B., Disseler, S.M., Kirby, B.J., Ratcliff, II, W., Shao, Q., Murata, K., Zhu, X., Yu, G., Fan, Y., Montazeri, M., Han, X., Borchers, J.A., Wang, K.L., "Tailoring Exchange Couplings in Magnetic Topological-Insulator/Antiferromagnet Heterostructures," Nat. Mater. 16(1), 94 (2017).

He, T., Pachfule, P., Wu, H., Xu, Q., Chen, P., "Hydrogen Carriers," Nat. Rev. Mater. 1(12), 16059 (2016).

Heacock, B., Arif, M., Haun, R., Huber, M.G., Pushin, D.A., Young, A.R., "Neutron Interferometer Crystallographic Imperfections and Gravitationally Induced Quantum Interference Measurements," Phys. Rev. A 95(1), 013840 (2017).
Heitmann, T., Lamsal, J., Watson, S., Erwin, R., Chen, W., Zhao, Y., Montfrooij, W., "Identification of the Low-Energy Excitations in a Quantum Critical System," AIP Advances 7(5), 055701 (2017).

Hester, G., Heitmann, T., Tyagi, M., Rathore, M., Dalvi, A., Mitra, S., "Neutron Scattering Studies of Lithium-Ion Diffusion in Ternary Phosphate Glasses," MRS Advances 1(45), 3057 (2016). [CHRNS]

Hoffman, J.D., Kirby, B.J., Kwon, J., Fabbris, G., Meyers, D., Freeland, J.W., Martin, I., Heinonen, O.G., Steadman, P., Zhou, H., Schlepütz, C.M., Dean, M.P.M., te Velthuis, S.G.E., Zuo, J.-M., Bhattacharya, A., "Oscillatory Noncollinear Magnetism Induced by Interfacial Charge Transfer in Superlattices Composed of Metallic Oxides," Phys. Rev. X 6(4), 041038 (2016).

Holewinski, A., Sakwa-Novak, M.A., Carrillo, J.-M.Y., Potter, M.E., Ellebracht, N., Rother, G., Sumpter, B.G., Jones, C.W., "Aminopolymer Mobility and Support Interactions in Silica-PEI Composites for $\mathrm{CO}_{2}$ Capture Applications: A Quasielastic Neutron Scattering Study," J. Phys. Chem. B 121, 6721 (2017). [CHRNS]

Hong, L., Jain, N., Cheng, X., Bernal, A., Tyagi, M., Smith, J.C., "Determination of Functional Collective Motions in a Protein at Atomic Resolution using Coherent Neutron Scattering," Sci. Adv. 2(10), e1600886 (2016). [CHRNS]

Hong, T., Qiu, Y., Matsumoto, M., Tennant, D.A., Coester, K., Schmidt, K.P., Awwadi, F.F., Turnbull, M.M., Agrawal, H., Chernyshev, A.L., "Field Induced Spontaneous Quasiparticle Decay and Renormalization of Quasiparticle Dispersion in a Quantum Antiferromagnet," Nat. Commun. 8, 15148 (2017). [CHRNS]

Hong, T., Matsumoto, M., Qiu, Y., Chen, W., Gentile, T.R., Watson, S., Awwadi, F.F., Turnbull, M.M., Dissanayake, S.E., Agrawal, H., Toft-Petersen, R., Klemke, B., Coester, K., Schmidt, K.P., Tennant, D.A., "Higgs Amplitude Mode in a Two-Dimensional Quantum Antiferromagnet near the Quantum Critical Point," Nat. Phys. 13(7), 638 (2017). [CHRNS]

Hoogerheide, D.P., Gurnev, P.A., Rostovtseva, T.K., Bezrukov, S.M., "Mechanism of $\alpha$-Synuclein Translocation through a VDAC Nanopore Revealed by Energy Landscape Modeling of Escape Time Distributions," Nanoscale 9(1), 183 (2017).

Hoogerheide, D.P., Noskov, S.Y., Jacobs, D., Bergdoll, L., Silin, V., Worcester, D.L., Abramson, J., Nanda, H., Rostovtseva, T.K., Bezrukov, S.M., "Structural Features and Lipid Binding Domain of Tubulin on Biomimetic Mitochondrial Membranes," P. Natl. A. Sci. USA 114(18), E3622 (2017). 
Horkay, F., Basser, P.J., Hecht, A.-M., Geissler, E., "Structure and Properties of Cartilage Proteoglycans," Macromol. Symp. 372(1), 43 (2017). [CHRNS]

Horkay, F., Basser, P.J., "Osmotic Properties of Cartilage," in "Biophysics and Biochemistry of Cartilage by NMR and MRI," edited by Xia, Y., and Momot, K.I., (Royal Society of ChemistryThomas Graham House, Cambridge, United Kingdom) Chap. 2, 44 (2016).

Howell, S.C., Qiu, X., Curtis, J.E., "Monte Carlo Simulation Algorithm for B-DNA," J. Comput. Chem. 37(29), 2553 (2016).

Hrabe, N., Gnäupel-Herold, T., Quinn, T., "Fatigue Properties of a Titanium Alloy (Ti-6Al-4V) Fabricated via Electron Beam Melting (EBM): Effects of Internal Defects and Residual Stress," Int. J. Fatigue 94(2), 202 (2017).

Hu, J., Xiao, Y., Tang, H., Wang, H., Wang, Z., Liu, C., Zeng, H., Huang, Q., Ren, Y., Wang, C., Zhang, W., Pan, F., "Tuning Li-lon Diffusion in $\alpha$-LiMn ${ }_{1-x} \mathrm{Fe}_{x} \mathrm{PO}_{4}$ Nanocrystals by Antisite Defects and Embedded $\beta$-Phase for Advanced Li-Ion Batteries," Nano Lett., in press.

Hu, W.-T., Yang, H., Cheng, H., Hu, H.-Q., "Morphology Evolution of Polystyrene-Core/Poly(N-Isopropylacrylamide)-Shell Microgel Synthesized by One-Pot Polymerization," Chinese J. Polym. Sci., in press. [CHRNS]

Hung, L., da Jornada, F.H., Souto-Casares, J., Chelikowsky, J.R., Louie, S.G., Öğüt, S., "Excitation Spectra of Aromatic Molecules within a Real-Space GW-BSE Formalism: Role of Self-Consistency and Vertex Corrections," Phys. Rev. B 94(8), 085125 (2016).

Hung, L., Bruneval, F., Baishya, K., Öğüt, S., "Benchmarking the GW Approximation and Bethe-Salpeter Equation for Groups IB and IIB Atoms and Monoxides," J. Chem. Theory Comput. 13(5), 2135 (2017).

Hussey, D.S., LaManna, J.M., Baltic, E., Jacobson, D.L., "Neutron Imaging Detector with $2 \mu \mathrm{m}$ Spatial Resolution Based on Event Reconstruction of Neutron Capture in Gadolinium Oxysulfide Scintillators," Nucl. Instrum. Meth. A, in press.

Hussey, D.S., LaManna, J.M., Baltic, E., Jacobson, D.L., "Neutron Imaging Detector with Scintillation Light Magnification and Amplification," ECS Transactions 75(14), 245 (2016).

Imai, T., Lee, Y.S., “Do Quantum Spin Liquids Exist?" Phys. Today 69(8), 30 (2016). [CHRNS]
Imel, A.E., Rostom, S., Holley, W., Baskaran, D., Mays, J.W., Dadmun, M.D., "The Tracer Diffusion Coefficient of Soft Nanoparticles in a Linear Polymer Matrix," RSC Adv. 7(25), 15574 (2017).

Ipek, B., Wulfers, M.J., Kim, H., Göltl, F., Hermans, I., Smith, J.P., Booksh, K.S., Brown, C.M., Lobo, R.F., "Formation of $\left[\mathrm{Cu}_{2} \mathrm{O}_{2}\right]^{2+}$ and $\left[\mathrm{Cu}_{2} \mathrm{O}\right]^{2+}$ Toward C-H Bond Activation in Cu-SSZ-13 and Cu-SSZ-39," ACS Catalysis 7(7), 4291 (2017).

Jamer, M.E., Sterbinsky, G.E., Stephen, G.M., DeCapua, M.C., Player, G., Heiman, D., "Magnetic Properties of Low-Moment Ferrimagnetic Heusler $\mathrm{Cr}_{2} \mathrm{CoGa}$ Thin Films Grown by Molecular Beam Epitaxy," Appl. Phys. Lett. 109(18), 182402 (2016).

Jamer, M.E., Wang, Y.J., Stephen, G.M., McDonald, I.J., Grutter, A.J., Sterbinsky, G.E., Arena, D.A., Borchers, J.A., Kirby, B.J., Lewis, L.H., Barbiellini, B., Bansil, A., Heiman, D., "Compensated Ferrimagnetism in the Zero-Moment Heusler Alloy $\mathrm{MN}_{3} \mathrm{Al}$," Phys. Rev. Appl. 7(6), 064036 (2017).

Janot, R., Tang, W.S., Clémençon, D., Chotard, J.-N., "Catalyzed $\mathrm{KSiH}_{3}$ as a Reversible Hydrogen Storage Material," J. Mater. Chem. A 4(48), 19045 (2016).

Jeong, Y., Gnäupel-Herold, T., ladicola, M., Creuziger, A., "Uncertainty in Flow Stress Measurements using X-ray Diffraction for Sheet Metals Subjected to Large Plastic Deformations," J. Appl. Crystallogr. 49, 1991 (2016).

Jiang, N., Sendogdular, L., Sen, M., Endoh, M.K., Koga, T., Fukuto, M., Akgun, B., Satija, S.K., Nam, C.-Y., "Novel Effects of Compressed $\mathrm{CO}_{2}$ Molecules on Structural Ordering and Charge Transport in Conjugated Poly(3-Hexylthiophene) Thin Films," Langmuir 32(42), 10851 (2016).

Jiang, Z., Heinrich, F., McGlinchey, R.P., Gruschus, J.M., Lee, J.C., "Segmental Deuteration of $\alpha$-Synuclein for Neutron Reflectometry on Tethered Bilayers," J. Phys. Chem. Lett. 8(1), 29 (2017).

Jones, S.Z., Bentz, D.P., Davis, J.M., Hussey, D.S., Jacobson, D.L., Molloy, J.L., Sieber, J.R., "Measurement and Modeling of the Ability of Crack Fillers to Prevent Chloride Ingress into Mortar," Cement Concrete Comp., in press.

Kamusella, S., Klauss, H.-H., Thakur, G.S., Haque, Z., Gupta, L.C., Ganguli, A.K., Kraft, I., Burkhardt, U., Rosner, H., Luetkens, H., Lynn, J.W., Zhao, Y., "Magnetism and Site Exchange in CuFeAs and CuFeSb: A Microscopic and Theoretical Investigation," Phys. Rev. B 95(9), 094415 (2017). 
Karna, S.K., Zhao, Y., Sankar, R., Avdeev, M., Tseng, P.C., Wang, C.W., Shu, G.J., Matan, K., Guo, G.Y., Chou, F.C., "Sodium Layer Chiral Distribution and Spin Structure of $\mathrm{Na}_{2} \mathrm{Ni}_{2} \mathrm{TeO}_{6}$ with a Ni Honeycomb Lattice," Phys. Rev. B 95(10), 104408 (2017).

Kawabata, Y., Bradbury, R., Kugizaki, S., Weigandt, K., Melnichenko, Y.B., Sadakane, K., Yamada, N.L., Endo, H., Nagao, M., Seto, H., "Effect of Interlamellar Interactions on Shear Induced Multilamellar Vesicle Formation," J. Chem. Phys. 147(3), 034905 (2017). [CHRNS]

Kawachi, S., Miyake, A., Ito, T., Dissanayake, S.E., Matsuda, M., Ratcliff, II, W., Xu, Z., Zhao, Y., Miyahara, S., Furukawa, N., Tokunaga, M., "Successive Field-Induced Transitions in $\mathrm{BiFeO}_{3}$ Around Room Temperature," Phys. Rev. Mater. 1(2), 024408 (2017).

Kim, J., Helgeson, M.E., "Shear-Induced Clustering of Brownian Colloids in Associative Polymer Networks at Moderate Péclet Number," Phys. Rev. Fluids 1(4), 043302 (2016).

Kline, S.R., "2016 NCNR Annual Report," NIST SP 1208, 1 (2017).

Koborinai, R., Dissanayake, S.E., Reehuis, M., Matsuda, M., Kajita, T., Kuwahara, H., Lee, S.-H., Katsufuji, T., "Orbital Glass State of the Nearly Metallic Spinel Cobalt Vanadate," Phys. Rev. Lett. 116(3), 037201 (2016)

Kofu, M., Hashimoto, N., Akiba, H., Kobayashi, H., Kitagawa, H., Kitagawa, H., Tyagi, M., Faraone, A., Copley, J.R.D., Lohstroh, W., Yamamuro, O., "Hydrogen Diffusion in Bulk and Nanocrystalline Palladium: A Quasielastic Neutron Scattering Study," Phys. Rev. B 94(6), 064303 (2016). [CHRNS]

Koh, M.L., FitzGerald, P.A., Warr, G.G., Jolliffe, K.A., Perrier, S., "Study of (Cyclic Peptide)-Polymer Conjugate Assemblies by Small-Angle Neutron Scattering," Chem.-Eur. J. 22(51), 18419 (2016). [CHRNS]

Konduru, V., Bellur, K., Médici, E.F., Allen, J.S., Choi, C.K., Hussey, D.S., Jacobson, D., Leão, J.B., McQuillen, J., Hermanson, J.C., "Examining Liquid Hydrogen Wettability using Neutron Imaging," J. Heat Transfer 138(8), 080901 (2016).

Koo, J., Satija, S., Lee, J.-S., Seo, Y.-S., "Spontaneous Bilayer Phase Separations of Spin-Coated Polymer Blend Thin Films: A Neutron Reflectivity Study," Macromol. Res. 24(12), 1105 (2016).

Koo, J., Kim, H., Kim, K.-Y., Jang, Y.R., Lee, J.-S., Yoon, S.W., Suh, B.J., Yu, T., Bang, J., Yoon, K., Yuan, G., Satija, S.K., "Controlling the Magnetic Properties of Polymer-Iron Oxide Nanoparticle Composite Thin Films via Spatial Particle Orientation," RSC Adv. 6(61), 55842 (2016).
Kuno, Y., Tassel, C., Fujita, K., Batuk, D., Abakumov, A.M., Shitara, K., Kuwabara, A., Moriwake, H., Watabe, D., Ritter, C., Brown, C.M., Yamamoto, T., Takeiri, F., Abe, R., Kobayashi, Y., Tanaka, K., Kageyama, H., "ZnTaO $2 \mathrm{~N}$ : Stabilized High-Temperature $\mathrm{LiNbO}_{3}$ Type Structure," J. Am. Chem. Soc. 138(49), 15950 (2016).

Kupwade-Patil, K., Tyagi, M., Brown, C.M., Büyüköztürk, O., "Water Dynamics in Cement Paste at Early Age Prepared with Pozzolanic Volcanic Ash and Ordinary Portland Cement using Quasielastic Neutron Scattering," Cement Concrete Res. 86, 55 (2016). [CHRNS]

Kwaambwa, H.M., Hellsing, M.S., Wasbrough, M.J., Bleuel, M., Rennie, A.R., "Salt Induced Polystyrene Latex Flocs Investigated by Neutron Scattering," J. Colloid Interf. Sci., in press. [CHRNS]

Lamm, R.J., Lim, E.B., Weigandt, K.M., Pozzo, L.D., White, N.J., Pun, S.H., "Peptide Valency Plays an Important Role in the Activity of a Synthetic Fibrin-Crosslinking Polymer," Biomaterials 132, 96 (2017). [CHRNS]

Lang, X., Lenart, W.R., Sun, J.E.P., Hammouda, B., Hore, M.J.A., "Interaction and Conformation of Aqueous Poly $(N$ Isopropylacrylamide) (PNIPAM) Star Polymers Below the LCST," Macromolecules 50(5), 2145 (2017). [CHRNS]

Larson, A.M., Wilfong, B., Moetakef, P., Brown, C.M., Zavalij, P., Rodriguez, E.E., "Metal-Insulator Transition Tuned by Magnetic Field in $\mathrm{Bi}_{1.7} \mathrm{~V}_{8} \mathrm{O}_{16}$ Hollandite," J. Mater. Chem. C 5(20), 4967 (2017).

Leão, J.B., Milner, J., "In-Situ Stress Measurement Comparison of Aluminum Pressure Vessels under High Working Pressures," J. Neutron Res. 19(1), 65 (2017).

Leão, J.B., Murphy, R.P., Wagner, N.J., Bleuel, M., "Dynamic Infrared Sample Controlled (DISCO) Temperature for the Tumbler Cells for Ultra Small Angle Neutron Scattering (USANS)," J. Neutron Res. 19(1), 23 (2017). [CHRNS]

Lee, C.-H., Wang, C.-W., Zhao, Y., Li, W.-H., Lynn, J.W., Harris, A.B., Rule, K., Yang, H.-D., Berger, H., "Complex Magnetic Incommensurability and Electronic Charge Transfer through the Ferroelectric Transition in Multiferroic $\mathrm{CO}_{3} \mathrm{TeO}_{6}$," Sci. Rep. 7(1), 6437 (2017).

Lee, P.W., Isarov, S.A., Wallat, J.D., Molugu, S.K., Shukla, S., Sun, J.E.P., Zhang, J., Zheng, Y., Dougherty, M.L., Konkolewicz, D., Stewart, P.L., Steinmetz, N.F., Hore, M.J.A., Pokorski, J.K., "Polymer Structure and Conformation Alter the Antigenicity of Virus-Like Particle-Polymer Conjugates," J. Am. Chem. Soc. 139(9), 3312 (2017). [CHRNS] 
Lehnert, R., Snow, W.M., Xiao, Z., Xu, R., "Constraining Spacetime Nonmetricity with Neutron Spin Rotation in Liquid ${ }^{4} \mathrm{He}$," Phys. Lett. $B$, in press.

Lenton, S., Grimaldo, M., Roosen-Runge, F., Schreiber, F., Nylander, T., Clegg, R., Holt, C., Härtlein, M., García Sakai, V., Seydel, T., Marujo Teixeira, S.C., "Effect of Phosphorylation on a HumanLike Osteopontin Peptide," Biophys. J. 112(8), 1586 (2017).

Leos, R., Wu, Z., Williams, R.E., Yang, X., "Integrating Sudo-Kaminaga Correlation to the Safety Analysis Code PARET-ANL," T. Am. Nucl. Soc. 116, 1369 (2017). [CHRNS]

Lesniewski, J.E., Disseler, S.M., Quintana, D.J., Kienzle, P.A., Ratcliff, W.D., "Bayesian Method for the Analysis of Diffraction Patterns using BLAND," J. Appl. Crystallogr. 49(6), 2201 (2016). [CHRNS]

Levin, I., Krayzman, V., Vanderah, T.A., Tomczyk, M., Wu, H., Tucker, M.G., Playford, H.Y., Woicik, J.C., Dennis, C.L., Vilarinho, P.M., "Oxygen-Storage Behavior and Local Structure in Ti-Substituted $\mathrm{YMnO}_{3}$," J. Solid State Chem. 246, 29 (2017).

Li, B., Wen, H.-M., Cui, Y., Zhou, W., Qian, G., Chen, B., “Emerging Multifunctional Metal-Organic Framework Materials," Adv. Mater. 28(40), 8819 (2016).

Li, B., Wen, H.-M., Zhou, W., Xu, J.Q., Chen, B., "Porous MetalOrganic Frameworks: Promising Materials for Methane Storage," Chem 1(4), 557 (2016).

Li, C.H., Lu, Q.M., Zhang, H.G., Yue, M., Wang, M.L., Zhang, D.T., Liu, D.M., Huang, Z.Q., "The Structure, Intrinsic Magnetic Properties, and Magnetic Hardening of $L 1_{0}-\mathrm{Mn}_{1.5} \mathrm{Ga}$ Alloy," J. Alloy Compd. 696, 604 (2017).

Li, D., Hsu, R., Figura, B., Jacobs, R., Li, S., Horvath, S., Clifford, T., Chari, K., "Rheology and Structure of Surface Crosslinked Surfactant-Activated Microgels," Soft Matter 12(34), 7150 (2016).

Li, H., Yang, Z., Pan, C., Jiang, N., Satija, S.K., Xu, D., Gersappe, D., Nam, C.-Y., Rafailovich, M.H., "A New Strategy to Engineer Polymer Bulk Heterojunction Solar Cells with Thick Active Layers via Self-Assembly of Tertiary Columnar Phase," Nanoscale, in press.

Li, J., Sleight, A.W., Subramanian, M.A., "Determination of the Local Environment of $\mathrm{Mn}^{3+}$ and $\mathrm{In}^{3+}$ in the $\mathrm{YlnO}_{3}-\mathrm{YMnO}_{3}$ Solid Solution, Which Exhibits an Intense Blue Color," Chem. Mater. 28(17), 6050 (2016).
Li, J., Medina, E.A., Stalick, J.K., Sleight, A.W., Subramanian, M.A., "Colored Oxides with Hibonite Structure: A Potential Route to Non-Cobalt Blue Pigments," Prog. Solid State Ch. 44(4), 107 (2016).

Li, J., Lorger, S., Stalick, J.K., Sleight, A.W., Subramanian, M.A., "From Serendipity to Rational Design: Tuning the Blue Trigonal Bipyramidal $\mathrm{Mn}^{3+}$ Chromophore to Violet and Purple through Application of Chemical Pressure," Inorg. Chem. 55(19), 9798 (2016).

Li, L., Lin, R.-B., Krishna, R., Wang, X., Li, B., Wu, H., Li, J., Zhou, W., Chen, B., "Flexible-Robust Metal-Organic Framework for Efficient Removal of Propyne from Propylene," J. Am. Chem. Soc. 139(23), 7733 (2017).

Li, L., Lin, R.-B., Krishna, R., Wang, X., Li, B., Wu, H., Li, J., Zhou, W., Chen, B., "Efficient Separation of Ethylene from Acetylene/ Ethylene Mixtures by a Flexible-Robust Metal-Organic Framework," J. Mater. Chem. A, in press.

Li, S., Xin, C., Liu, X., Feng, Y., Liu, Y., Zheng, J., Liu, F., Huang, Q., Qiu, Y., He, J., Luo, J., Pan, F., "2D Hetero-Nanosheets to Enable Ultralow Thermal Conductivity by all Scale Phonon Scattering for Highly Thermoelectric Performance," Nano Energy 30, 780 (2016).

Li, X., Wang, X., Han, Y., Jing, X., Huang, Q., Kuang, X., Gao, Q., Chen, J., Xing, $X .$, "High-Dielectric-Permittivity Layered Nitride CaTiN $_{2}$," Chem. Mater. 29(5), 1989 (2017).

Lin, R.-B., Li, L., Wu, H., Arman, H., Li, B., Lin, R.-G., Zhou, W., Chen, B., "Optimized Separation Acetylene from Carbon Dioxide and Ethylene in a Microporous Material," J. Am. Chem. Soc. 139(23), 8022 (2017).

Lindstrom, R.M., "Believable Statements of Uncertainty and Believable Science," J. Radioanal. Nucl. Ch. 311(2), 1019 (2017).

Lindstrom, R.M., "A Second-Generation Low-Background Gamma-Ray Spectrometer," Appl. Radiat. Isotopes, in press.

Liu, H., Chen, F., Bai, D., Jiao, J., Zhou, W., Yildirim, T., He, Y., "HighPressure Methane Adsorption in Two Isoreticular Zr-Based Metal-Organic Frameworks Constructed from $\mathrm{C}_{3}$-Symmetrical Tricarboxylates," Cryst. Growth Des. 17(1), 248 (2017).

Liu, M., Lin, L., Zhang, Y., Li, S., Huang, Q., Ovidiu Garlea,V., Zou, T., Xie, Y., Wang, Y., Lu, C., Yang, L., Yan, Z., Wang, X., Dong, S., Liu, J.-M., "Cycloidal Magnetism Driven Ferroelectricity in Double Tungstate LiFe $\left(\mathrm{WO}_{4}\right)_{2}$," Phys. Rev. B 95(19), 195134 (2017). 
Liu, M., Chen, L., Lewis, S., Chong, S.Y., Little, M.A., Hasell, T., Aldous, I.M., Brown, C.M., Smith, M.W., Morrison, C.A., Hardwick, L.J., Cooper, A.I., "Three-Dimensional Protonic Conductivity in Porous Organic Cage Solids," Nat. Commun. 7, 12750 (2016). [CHRNS]

Liu, Y., "Intermediate Scattering Function for Macromolecules in Solutions Probed by Neutron Spin Echo," Phys. Rev. E 95(2), 020501 (2017). [CHRNS]

Liu, Z., Huang, J., Tyagi, M., O'Neill, H., Zhang, Q., Mamontov, E., Jain, N., Wang, Y., Zhang, J., Smith, J.C., Hong, L., "Dynamical Transition of Collective Motions in Dry Proteins," Phys. Rev. Lett. 119(4), 048101 (2017). [CHRNS]

Longanecker, M., Modi, A., Dobrynin, A., Kim, S., Yuan, G., Jones, R., Satija, S., Bang, J., Karim, A., "Reduced Domain Size and Interfacial Width in Fast Ordering Nanofilled Block Copolymer Films by Direct Immersion Annealing," Macromolecules 49(22), 8563 (2016).

López-Barrón, C.R., Zeng, Y., Richards, J.J., "Chain Stretching and Recoiling During Startup and Cessation of Extensional Flow of Bidisperse Polystyrene Blends," J. Rheol. 61(4), 697 (2017).

López-Barrón, C.R., Zeng, Y., Schaefer, J.J., Eberle, A.P.R., Lodge, T.P., Bates, F.S., "Molecular Alignment in Polyethylene During Cold Drawing using In-Situ SANS and Raman Spectroscopy," Macromolecules 50(9), 3627 (2017).

Lu, X., "Short-Range Cluster Spin Glass Near Optimal Superconductivity in $\mathrm{BaFe}_{2-x} \mathrm{Ni}_{x} \mathrm{As}_{2}$," in "Phase Diagram and Magnetic Excitations of $\mathrm{BaFe}_{2-x} \mathrm{Ni}_{x} \mathrm{As}_{2}$ : A Neutron Scattering Study," (Springer, Singapore, Singapore) Chap. 5, 67 (2017).

Lucero, C.L., Spragg, R.P., Bentz, D.P., Hussey, D.S., Jacobson, D.L., Weiss, W.J., "Neutron Radiography Measurement of Salt Solution Absorption in Mortar," ACI Mater. J. 114(1), 149 (2017).

Luhmann, A.J., Tutolo, B.M., Bagley, B.C., Mildner, D.F.R., Scheuermann, P., Feinberg, J.M., Ignatyev, K., Seyfried, Jr., W.E., "Chemical and Physical Changes During Seawater Flow through Intact Dunite Cores: An Experimental study at 150-200 ${ }^{\circ} \mathrm{C}$," Geochim. Cosmochim. Ac., in press. [CHRNS]

Luhmann, A.J., Tutolo, B.M., Bagley, B.C., Mildner, D.F.R., Seyfried, Jr., W.E., Saar, M.O., "Permeability, Porosity, and Mineral Surface Area Changes in Basalt Cores Induced by Reactive Transport of $\mathrm{CO}_{2}$-Rich Brine," Water Resour. Res. 53(3), 1908 (2016). [CHRNS]
Lumsden, M.D., Fernandez-Baca, J.A., Helgeson, M.E., Jones, R.L., Lynn, J.W., Page, K., Smith, G.S., Tucker, M., Wagner, N.J., "The 8th American Conference on Neutron Scattering," Neutron News 27(4), 4 (2016).

Luo, H., Szymusiak, M., Garcia, E.A., Lock, L.L., Cui, H., Liu, Y., HerreraAlonso, M., "Solute-Triggered Morphological Transitions of an Amphiphilic Heterografted Brush Copolymer as a SingleMolecule Drug Carrier," Macromolecules 50(5), 2201 (2017).

Luo, J., Yuan, G., Han, C.C., "Tuning the Bridging Attraction between Large Hard Particles by the Softness of Small Microgels," Soft Matter 12(37), 7863 (2016). [CHRNS]

Ma, Y., Lodge, T.P., "Chain Exchange Kinetics in Diblock Copolymer Micelles in lonic Liquids: The Role of $\chi{ }^{\prime \prime}$ Macromolecules 49(24), 9542 (2016).

MacEwan, S.R., Weitzhandler, I., Hoffmann, I., Genzer, J., Gradzielski, M., Chilkoti, A., "Phase Behavior and Self-Assembly of Perfectly Sequence-Defined and Monodisperse Multiblock Copolypeptides," Biomacromolecules 18(2), 599 (2017). [CHRNS]

Macauley, N., Lujan, R.W., Spernjak, D., Hussey, D.S., Jacobson, D.L., More, K., Borup, R.L., Mukundan, R., "Durability of Polymer Electrolyte Membrane Fuel Cells Operated at Subfreezing Temperatures," J. Electrochem. Soc. 163(13), F1317 (2016).

Magiera, J., "Experimental-Numerical Study of Manufacture Induced 3D Rail Residual Stress," Solid State Phenomena 240, 17 (2015).

Malo de Molina, P., Zhang, M., Bayles, A.V., Helgeson, M.E., "Oil-inWater-in-Oil Multinanoemulsions for Templating Complex Nanoparticles," Nano Lett. 16(12), 7325 (2016). [CHRNS]

Man, H., Guo, J., Zhang, R., Schönemann, R., Yin, Z., Fu, M., Stone, M.B., Huang, Q., Song, Y., Wang, W., Singh, D.J., Lochner, F., Hickel, T., Eremin, I., Harriger, L., Lynn, J.W., Broholm, C., Balicas, L., Si, Q., Dai, P., "Spin Excitations and the Fermi Surface of Superconducting FeS," NPJ Quantum Mater. 2(14), 1 (2017). [CHRNS]

Manley, M.E., Abernathy, D.L., Sahul, R., Parshall, D.E., Lynn, J.W., Christianson, A.D., Stonaha, P.J., Specht, E.D., Budai, J.D., "Giant Electromechanical Coupling of Relaxor Ferroelectrics Controlled by Polar Nanoregion Vibrations," Sci. Adv. 2(9), e1501814 (2016).

Mao, H., Wang, C., Maynard-Casely, H.E., Huang, Q., Wang, Z., Cao, G., Li, S., Luo, H., "Neutron Powder Diffraction Study on the Iron-Based Nitride Superconductor ThFeAsN," Europhys. Lett. 117(5), 57005 (2017). 
Mao, Y., Kriegel, R.M., Bucknall, D.G., "The Crystal Structure of Poly(Ethylene Furanoate)," Polymer 102, 308 (2016).

Mao, Y., Su, Y., Hsiao, B.S., "Probing Structure and Orientation in Polymers using Synchrotron Small-and Wide-Angle X-ray Scattering Techniques," Eur. Polym. J. 81, 433 (2016).

Mao, Y., Liu, K., Zhan, C., Geng, L., Chu, B., Hsiao, B.S., "Characterization of Nanocellulose using Small-Angle Neutron, X-ray, and Dynamic Light Scattering Techniques," J. Phys. Chem. B 121(6), 1340 (2017).

Maranville, B.B., "Interactive, Web-Based Calculator of Neutron and X-ray Reflectivity," J. Res. Natl. Inst. Stan. 122, 1 (2017).

Maranville, B.B., Kirby, B.J., Grutter, A.J., Kienzle, P.A., Majkrzak, C.F., Liu, Y., Dennis, C.L., "Measurement and Modeling of Polarized Specular Neutron Reflectivity in Large Magnetic Fields," J. Appl. Crystallogr. 49(4), 1121 (2016).

Masoomi, M., Shamsaei, N., Winholtz, R.A., Milner, J., GnäupelHerold, T., Elwany, A., Mahmoudi, M., Thompson, S.M., "Residual Stress Measurements via Neutron Diffraction of Additive Manufactured Stainless Steel 17-4 PH," Data in Brief, in press.

Matsuda, M., Onishi, H., Okutani, A., Ma, J., Agrawal, H., Hong, T., Pajerowski, D.M., Copley, J.R.D., Okunishi, K., Mori, M., Kimura, S., Hagiwara, M., "Magnetic Structure and Dispersion Relation of the $S=1 / 2$ Quasi-One-Dimensional Ising-Like Antiferromagnet $\mathrm{BaCO}_{2} \mathrm{~V}_{2} \mathrm{O}_{8}$ in a Transverse Magnetic Field," Phys. Rev. B 96(2), 024439 (2017).

Mazzei, L., Wolff, M., Pergolesi, D., Dura, J.A., Börjesson, L., Gutfreund, P., Bettinelli, M., Lippert, T., Karlsson, M., "Structure and Conductivity of Epitaxial Thin Films of In-Doped $\mathrm{BaZrO}_{3}$ Based Proton Conductors," J. Phys. Chem. C 120(50), 28415 (2016).

Mazzer, A.R., Clifton, L.A., Perevozchikova, T., Butler, P.D., Roberts, C.J., Bracewell, D.G., "Neutron Reflectivity Measurement of Protein A-Antibody Complex at the SolidLiquid Interface," J. Chromatogr. A 1499, 118 (2017).

Medina, E.A., Li, J., Subramanian, M.A., "Colored Oxides with Hibonite Structure II: Structural and Optical Properties of $\mathrm{CaAl}_{12} \mathrm{O}_{19}$-Type Pigments with Chromophores Based on Fe, Mn, Cr and Cu," Prog. Solid State Ch. 45-46, 9 (2017).

Menéndez, E., Modarresi, H., Petermann, C., Nogués, J., Domingo, N., Liu, H., Kirby, B.J., Mohd, A.S., Salhi, Z., Babcock, E., Mattauch, S., Van Haesendonck, C., Vantomme, A., Temst, K., "Lateral Magnetically Modulated Multilayers by Combining Ion Implantation and Lithography," Small 13(11), 1603465 (2017).
Merritt, A., Rodriguez-Rivera, J., Li, Y., Wang, W., Zhang, C., Dai, P., Reznik, D., "Absence of Long-Wavelength Nematic Fluctuations in LiFeAs," J. Supercond. Nov. Magn. 29(12), 3049 (2016). [CHRNS]

Meynell, S.A., Wilson, M.N., Krycka, K.L., Kirby, B.J., Fritzsche, H., Monchesky, T.L., "Neutron Study of In-Plane Skyrmions in MnSi Thin Films," Phys. Rev. B, in press.

Michor, E.L., Ponto, B.S., Berg, J.C., "Effects of Reverse Micellar Structure on the Particle Charging Capabilities of the Span Surfactant Series," Langmuir 32(40), 10328 (2016).

Middleton, L.R., Tarver, J.D., Cordaro, J., Tyagi, M., Soles, C.L., Frischknecht, A.L., Winey, K.I., "Heterogeneous Chain Dynamics and Aggregate Lifetimes in Precise Acid-Containing Polyethylenes: Experiments and Simulations," Macromolecules 49(23), 9176 (2016). [CHRNS]

Mills, C.E., Obermeyer, A., Dong, X., Walker, J., Olsen, B.D., "Complex Coacervate Core Micelles for the Dispersion and Stabilization of Organophosphate Hydrolase in Organic Solvents," Langmuir 32(50), 13367 (2016). [CHRNS]

Mineart, K.P., Kelley, E.G., Nagao, M., Prabhu, V.M., "ProcessingStructure Relationships of Poly(Ethylene Glycol)-Modified Liposomes," Soft Matter, in press. [CHRNS]

Mink, J., Lin, Y.-C., Karlsson, M., Österberg, C., Udovic, T.J., Fahlquist, H., Häussermann, U., "Vibrational Properties of $\beta-\mathrm{KSiH}_{3}$ and $\beta-\mathrm{RbSiH}_{3}$ : A Combined Raman and Inelastic Neutron Scattering Study," J. Raman Spectrosc. 48(2), 284 (2017).

Minns, J.L., Zajdel, P., Chernyshov, D., van Beek, W., Green, M.A., "Structure and Interstitial lodide Migration in Hybrid Perovskite Methylammonium Lead Iodide," Nat. Commun. 8, 15152 (2017).

Miskowiec, A., Buck, Z.N., Hansen, F.Y., Kaiser, H., Taub, H., Tyagi, M., Diallo, S.O., Mamontov, E., Herwig, K.W., "On the Structure and Dynamics of Water Associated with Single-Supported Zwitterionic and Anionic Membranes," J. Chem. Phys. 146(12), 125102 (2017). [CHRNS]

Morgan, B., Dadmun, M.D., "Illumination Alters the Structure of Gels Formed from the Model Optoelectronic Material P3HT," Polymer 108, 313 (2017). [CHRNS]

Morgan, B., Dadmun, M.D., "The Role of Incident Light Intensity, Wavelength, and Exposure Time in the Modification of Conjugated Polymer Structure in Solution," Eur. Polym. J. 89, 272 (2017). [CHRNS] 
Morgan, B., Dadmun, M.D., "The Effect of Illumination on the Depth Profile of Thermally Annealed MEH-PPV/dPS Blends," J. Polym. Sci. Pol. Phys., in press.

Morgan, B., Dadmun, M.D., "The Importance of Solvent Quality on the Modification of Conjugated Polymer Conformation and Thermodynamics with Illumination," Soft Matter 13(15), 2773 (2017). [CHRNS]

Motokawa, R., Endo, H., Nagao, M., Heller, W.T., "Neutron Polarization Analysis for Biphasic Solvent Extraction Systems," Solvent Extr. Ion Exc. 34(5), 399 (2016). [CHRNS]

Mundy, J.A., Brooks, C.M., Holtz, M.E., Moyer, J.A., Das, H., Rébola, A.F., Heron, J.T., Clarkson, J.D., Disseler, S.M., Liu, Z., Farhan, A., Held, R., Hovden, R., Padgett, E., Mao, Q., Paik, H., Misra, R., Kourkoutis, L.F., Arenholz, E., Scholl, A., Borchers, J.A., Ratcliff, W.D., Ramesh, R., Fennie, C.J., Schiffer, P., Muller, D.A., Schlom, D.G., "Atomically Engineered Ferroic Layers Yield a Room-Temperature Magnetoelectric Multiferroic," Nature 537(7621), 523 (2016).

Nagy-Smith, K., Beltramo, P.J., Moore, E., Tycko, R., Furst, E.M., Schneider, J.P., "Molecular, Local, and Network-Level Basis for the Enhanced Stiffness of Hydrogel Networks Formed from Coassembled Racemic Peptides: Predictions from Pauling and Corey," ACS Central Science 3(6), 586 (2017). [CHRNS]

Nandjou, F., Poirot-Crouvezier, J.-P., Chandesris, M., Rosini, S., Hussey, D.S., Jacobson, D.L., LaManna, J.M., Morin, A., Bultel, Y., "A Pseudo-3D Model to Investigate Heat and Water Transport in Large Area PEM Fuel Cells - Part 2: Application on an Automotive Driving Cycle," Int. J. Hydrogen Energ. 41(34), 15573 (2016).

Natarajan, B., Orloff, N.D., Ashkar, R., Doshi, S., Twedt, K., Krishnamurthy, A., Davis, C., Forster, A.M., Thostenson, E., Obrzut, J., Sharma, R., Liddle, J.A., "Multiscale Metrologies for Process Optimization of Carbon Nanotube Polymer Composites," Carbon 108, 381 (2016). [CHRNS]

Nedumkandathil, R., Jaworski, A., Fischer, A., Österberg, C., Lin, Y.-C., Karlsson, M., Grins, J., Pell, A.J., Edén, M., Häussermann, U., "Investigation of the Order-Disorder Rotator Phase Transition in $\mathrm{KSiH}_{3}$ and $\mathrm{RbSiH}_{3}$," J. Phys. Chem. C 121(9), 5241 (2017).

Neer, A.J., Milam-Guerrero, J., So, J.E., Melot, B.C., Ross, K.A., Hulvey, Z., Brown, C.M., Sokol, A.A., Scanlon, D.O., "Ising-Like Antiferromagnetism on the Octahedral Sublattice of a CobaltContaining Garnet and the Potential for Quantum Criticality," Phys. Rev. B 95(14), 144419 (2017).
Newbloom, G.M., Kanekal, K., Richards, J.J., Pozzo, L.D., "Characterization of Polymer Semiconductors by Neutron Scattering Techniques," in "RSC Polymer Chemistry Series No. 21 Semiconducting Polymers: Controlled Synthesis and Microstructure," edited by Luscombe, C. (The Royal Society of Chemistry - Thomas Graham House, Cambridge, United Kingdom) Chap. 5, 165 (2016). [CHRNS]

Nouhi, S., Hellsing, M.S., Kapaklis, V., Rennie, A.R., "GrazingIncidence Small-Angle Neutron Scattering from Structures Below an Interface," J. Appl. Crystallogr., in press. [CHRNS]

Nsofini, J., Sarenac, D., Ghofrani, K., Huber, M.G., Arif, M., Cory, D.G., Pushin, D.A., "Noise Refocusing in a Five-Blade Neutron Interferometer," J. Appl. Phys., in press.

O’Neil, L., Andenoro, K., Pagano, I., Carroll, L., Langer, L., Dell, Z., Perera, D., Treece, B.W., Heinrich, F., Lösche, M., Nagle, J.F., Tristram-Nagle, S., "HIV-1 Matrix-31 Membrane Binding Peptide Interacts Differently with Membranes Containing PS vs. PI(4,5) $\mathrm{P}_{2}$," Biochim. Biophys. Acta, Biomembr. 1858(12), 3071 (2016).

Oredeko, A., Yang, X., Wu, Z., "MOCUM Verification with a Heterogeneous MOX Whole Core C5G7 Benchmark Problem," T. Am. Nucl. Soc. 115, 1344 (2016).

Osti, N.C., Etampawala, T.N., Shrestha, U.M., Aryal, D., Tyagi, M., Diallo, S.O., Mamontov, E., Cornelius, C.J., Perahia, D., "Water Dynamics in Rigid lonomer Networks," J. Chem. Phys. 145(22), 224901 (2016). [CHRNS]

Osti, N.C., Naguib, M., Tyagi, M., Gogotsi, Y., Kolesnikov, A.I., Mamontov, E., "Evidence of Molecular Hydrogen Trapped in Two-Dimensional Layered Titanium Carbide-Based MXene," Phys. Rev. Mater. 1(2), 024004 (2017). [CHRNS]

Paddison, J.A.M., Ong, H.S., Hamp, J.O., Mukherjee, P., Bai, X., Tucker, M.G., Butch, N.P., Castelnovo, C., Mourigal, M., Dutton, S.E., "Emergent Order in the Kagome Ising Magnet $\mathrm{Dy}_{3} \mathrm{Mg}_{2} \mathrm{Sb}_{3} \mathrm{O}_{14}, "$ Nat. Commun. 7, 13842 (2016).

Park, S-Y., Do, S.-H., Choi, K.-Y., Kang, J.-H., Jang, D., Schmidt, B., Brando, M., Kim, B.-H., Kim, D.-H., Butch, N.P., Lee, S., Park, J.-H., Ji, S., "Spin-Orbit Coupled Molecular Quantum Magnetism Realized in Inorganic Solid," Nat. Commun. 7, 12912 (2016).

Paul, R.L., "Prompt Gamma-Ray Activation Analysis for Certification of Sulfur in Fuel Oil SRMs," J. Radioanal. Nucl. Ch. 311(2), 1149 (2017). 
Pei, W.-Y., Xu, G., Yang, J., Wu, H., Chen, B., Zhou, W., Ma, J.-F., "Versatile Assembly of Metal-Coordinated Calix[4]resorcinarene Cavitands and Cages through Ancillary Linker Tuning," J. Am. Chem. Soc. 139(22), 7648 (2017).

Perkins, S.J., Wright, D.W., Zhang, H., Brookes, E.H., Chen, J., Irving, T.C., Krueger, S., Barlow, D.J., Edler, K.J., Scott, D.J., Terrill, N.J., King, S.M., Butler, P.D., Curtis, J.E., "Atomistic Modelling of Scattering Data in the Collaborative Computational Project for Small Angle Scattering (CCP-SAS)," J. Appl. Crystallogr. 49(6), 1861 (2016).

Pesek, S.L., Xiang, Q., Hammouda, B., Verduzco, R., "Small-Angle Neutron Scattering Analysis of Bottlebrush Backbone and Side Chain Flexibility," J. Polym. Sci. Pol. Phys. 55(1), 104 (2017). [CHRNS]

Pham, T.D., Hudson, M.R., Brown, C.M., Lobo, R.F., "On the Structure-Property Relationships of Cation-Exchanged ZK-5 Zeolites for $\mathrm{CO}_{2}$ Adsorption," ChemSusChem 10(5), 946 (2017).

Plumb, K.W., Morey, J.R., Rodriguez-Rivera, J.A., Wu, H., Podlesnyak, A.A., McQueen, T.M., Broholm, C.L., "Antiferromagnetic and Orbital Ordering on a Diamond Lattice Near Quantum Criticality," Phys. Rev. X 6(4), 041055 (2016). [CHRNS]

Poling-Skutvik, R., Mongcopa, K.I.S., Faraone, A., Narayanan, S., Conrad, J.C., Krishnamoorti, R., "Structure and Dynamics of Interacting Nanoparticles in Semidilute Polymer Solutions," Macromolecules 49(17), 6568 (2016). [CHRNS]

Pool, C.A., Ceballos, P.O., del Carmen Rodríguez, M., Sears, E.L., Bishop, R.L., Blackman, M.J., "An Early Olmec Manifestation in Western Olman," in "The Early Olmec and Mesoamerica,", edited by Blomster, J.P. and Cheetham, D., (Cambridge University Press, New York, New York) Chap. 4, 89 (2017).

Poudel, N., Acar, M., Tran, T., Xu, J., "An Experimental and Numerical Study of Convective Boiling of Nanoemulsion Inside MiniChannels Heat Exchanger," in "Heat Transfer and Thermal Engineering," edited by ASME, (ASME 2016 International Mechanical Engineering Congress and Exposition, October 2016, Phoenix, AZ) 8, V008T10A079 (2016).

Preta, G., Jankunec, M., Heinrich, F., Griffin, S., Sheldon, I.M., Valincius, G., "Tethered Bilayer Membranes as a Complementary Tool for Functional and Structural Studies: The Pyolysin Case," Biochim. Biophys. Acta, Biomembr. 1858(9), 2070 (2016).

Prisk, T.R., Bryan, M.S., Sokol, P.E., Granroth, G.E., Moroni, S., Boninsegni, M., "The Momentum Distribution of Liquid ${ }^{4} \mathrm{He}$," J. Low Temp. Phys., in press.
Pushin, D.A., Sarenac, D., Hussey, D.S., Miao, H., Arif, M., Cory, D.G., Huber, M.G., Jacobson, D.L., LaManna, J.M., Parker, J.D., Shinohara, T., Ueno, W., Wen, H., "Far-Field Interference of a Neutron White Beam and the Applications to Noninvasive Phase-Contrast Imaging," Phys. Rev. A 95(4), 043637 (2017).

Qu, G., Zhao, X., Newbloom, G.M., Zhang, F., Mohammadi, E., Strzalka, J.W., Pozzo, L.D., Mei, J., Diao, Y., "Understanding Interfacial Alignment in Solution Coated Conjugated Polymer Thin Films," ACS Appl. Mater. Interfaces, in press. [CHRNS]

Ragaliauskas, T., Mickevicius, M., Rakovska, B., Penkauskas, T., Vanderah, D.J., Heinrich, F., Valincius, G., "Fast Formation of Low-Defect-Density Tethered Bilayers by Fusion of Multilamellar Vesicles," Biochem. Biophys. Acta 1859(5), 669 (2017).

Rawl, R., Ge, L., Agrawal, H., Kamiya, Y., Dela Cruz, C.R., Butch, N.P., Sun, X.F., Lee, M., Choi, E.S., Oitmaa, J., Batista, C.D., Mourigal, M., Zhou, H.D., Ma, J., " $\mathrm{Ba}_{8} \mathrm{CoNb}_{6} \mathrm{O}_{24}$ : A Spin-1/2 Triangular-Lattice Heisenberg Antiferromagnet in the Two-Dimensional Limit," Phys. Rev. B 95(6), 060412 (2017).

Rezhdo, O., Di Maio, S., Le, P., Littrell, K.C., Carrier, R.L., Chen, S.-H., "Characterization of Colloidal Structures During Intestinal Lipolysis using Small-Angle Neutron Scattering," J. Colloid Interf. Sci., in press. [CHRNS]

Richards, J.J., Scherbarth, A.D., Wagner, N.J., Butler, P.D., "Mixed Ionic/Electronic Conducting Surface Layers Adsorbed on Colloidal Silica for Flow Battery Applications," ACS Appl. Mater. Interfaces 8(36), 24089 (2016). [CHRNS]

Richards, J.J., Gagnon, C.V.L., Krzywon, J.R., Wagner, N.J., Butler, P.D., "Dielectric RheoSANS - Simultaneous Interrogation of Impedance, Rheology and Small Angle Neutron Scattering of Complex Fluids," Jove-J. Vis. Exp. 122, e55318 (2017).

Richter, A.G., Dergunov, S.A., Kim, M.D., Shmakov, S.N., Pingali, S.V., Urban, V.S., Liu, Y., Pinkhassik, E., "Unraveling the SingleNanometer Thickness of Shells of Vesicle-Templated Polymer Nanocapsules," J. Phys. Chem. Lett., in press.

Riiff, T.J., Webb, M.A., Orts, W., Aramthanapon, K., "Small-Angle Neutron Scattering Studies on an Idealized Diesel Biofuel Platform," Energ. Fuel. 31(4), 3995 (2017). [CHRNS]

Ross, K.A., Brown, J.M., Cava, R.J., Krizan, J.W., Nagler, S.E., Rodriguez-Rivera, J.A., Stone, M.B., "Single-lon Properties of the $\mathrm{S}_{\text {eff }}=1 / 2 \mathrm{XY}$ Antiferromagnetic Pyrochlores $\mathrm{NaA}^{\prime} \mathrm{Co}_{2} \mathrm{~F}_{7}$ $\left(A^{\prime}=\mathrm{Ca}^{2+}, \mathrm{Sr}{ }^{2+}\right),{ }^{\prime \prime}$ Phys. Rev. B 95(14), 144414 (2017). [CHRNS] 
Rostovtseva, T.K., Hoogerheide, D.P., Rovini, A., Bezrukov, S.M., "Lipids in Regulation of the Mitochondrial Outer Membrane Permeability, Bioenergetics, and Metabolism," in "Molecular Basis for Mitochondrial Signaling," edited by Rostovtseva, T.K. (Springer International, Bethesda, Maryland) Chap. 8, 185 (2017)

Rubinson, K.A., Chen, Y., Cress, B.F., Zhang, F., Linhardt, R.J., "Heparin's Solution Structure Determined by Small-Angle Neutron Scattering," Biopolymers 105(12), 905 (2016). [CHRNS]

Rubinson, K.A., "Practical Corrections for $p(H, D)$ Measurements in Mixed $\mathrm{H}_{2} \mathrm{O} / \mathrm{D}_{2} \mathrm{O}$ Biological Buffers," Anal. Methods-UK 9(18), 2744 (2017)

Russina, O., Lo Celso, F., Plechkova, N.V., Triolo, A., "Emerging Evidences of Mesoscopic-Scale Complexity in Neat lonic Liquids and their Mixtures," J. Phys. Chem. Lett. 8(6), 1197 (2017). [CHRNS]

Saggu, P., Mineeva, T., Arif, M., Cory, D.G., Haun, R., Heacock, B., Huber, M.G., Li, K., Nsofini, J., Sarenac, D., Shahi, C.B., Skavysh, V., Snow, W.M., Werner, S.A., Young, A.R., Pushin, D.A., "Decoupling of a Neutron Interferometer from Temperature Gradients," Rev. Sci. Instrum. 87(12), 123507 (2016).

Şahin, D., MacDavid, S.J., "Managing Systematic Errors in the NBSR Thermal Power Calorimetric Measurements," in "Trans. Am. Nucl. Soc., USA, 2017," edited by ANS, (Trans. Am. Nucl. Soc., USA, 2017, May 2017, San Francisco, CA), 1 (2017).

Şahin, D., "NBSR Reactor Control Room Upgrade," in "Trans. Am. Nucl. Soc., USA, 2017," edited by ANS, (2017 ANS Annual Meeting, May 2017, San Francisco, CA),1 (2017).

Sales, B.C., Jin, K., Bei, H., Nichols, J., Chisholm, M.F., May, A.F., Butch, N.P., Christianson, A.D., McGuire, M.A., "Quantum Critical Behavior in the Asymptotic Limit of High Disorder in the Medium Entropy Alloy $\mathrm{NiCoCr}_{0.8}, "$ NPJ Quantum Mater. 2(1), 33 (2017).

Sarenac, D., Huber, M.G., Heacock, B., Arif, M., Clark, C.W., Cory, D.G., Shahi, C.B., Pushin, D.A., "Holography with a Neutron Interferometer," Opt. Express 24(20), 22528 (2016).

Schantz, A.B., Saboe, P.O., Sines, I.T., Lee, H.-Y., Bishop, K.J.M., Maranas, J.K., Butler, P.D., Kumar, M., "PEE-PEO Block Copolymer Exchange Rate between Mixed Micelles is Detergent and Temperature Activated," Macromolecules 50(6), 2484 (2017). [CHRNS]
Scherger, J.D., Evans, E.A., Dura, J.A., Foster, M.D., "Extending Nanoscale Spectroscopy with Titanium Nitride Probes," J. Raman Spectrosc. 47(11), 1332 (2016).

Sears, J.A., Zhao, Y., Xu, Z., Lynn, J.W., Kim, Y.-J., "Phase Diagram of $\alpha-\mathrm{RuCl}_{3}$ in an In-Plane Magnetic Field," Phys. Rev. B 95(18), 180411 (2017).

Senses, E., Ansar, S.M., Kitchens, C.L., Mao, Y., Narayanan, S., Natarajan, B., Faraone, A., "Small Particle Driven Chain Disentanglements in Polymer Nanocomposites," Phys. Rev. Lett. 118(14), 147801 (2017). [CHRNS]

Sergeicheva, E.G., Sosin, S.S., Prozorova, L.A., Gu, G.D., Zaliznyak, I.A., "Unusual Magnetic Excitations in the Weakly Ordered Spin-1/2 Chain Antiferromagnet $\mathrm{Sr}_{2} \mathrm{CuO}_{3}$ : Possible Evidence for Goldstone Magnon Coupled with the Amplitude Mode," Phys. Rev. B 95(2), 020411 (2017).

Sharma, V.K., Mamontov, E., Ohl, M., Tyagi, M., "Incorporation of Aspirin Modulates the Dynamical and Phase Behavior of the Phospholipid Membrane," Phys. Chem. Chem. Phys. 19(3), 2514 (2017). [CHRNS]

Sharma, V.K., Hayes, D.G., Urban, V.S., O’Neill, H.M., Tyagi, M., Mamontov, E., "Nanoscopic Dynamics of Bicontinuous Microemulsions: Effect of Membrane Associated Protein," Soft Matter 13(28), 4871 (2017).

Sheckelton, J.P., Plumb, K.W., Trump, B.A., Broholm, C.L., McQueen, T.M., "Rearrangement of van der Waals Stacking and Formation of a Singlet State at $T=90 \mathrm{~K}$ in a Cluster Magnet," Inorg. Chem. Front. 4(3), 481 (2017).

Shelton, C.K., Jones, R.L., Epps, III, T.H., "Kinetics of Domain Alignment in Block Polymer Thin Films During Solvent Vapor Annealing with Soft Shear: An In Situ Small-Angle Neutron Scattering Investigation," Macromolecules 50(14), 5367 (2017).

Shelton, C.K., Jones, R.L., Dura, J.A., Epps, III, T.H., "Tracking Solvent Distribution in Block Polymer Thin Films During Solvent Vapor Annealing with In Situ Neutron Scattering," Macromolecules 49(19), 7525 (2016).

Shen, Y., Li, Y.-D., Wo, H., Li, Y., Shen, S., Pan, B., Wang, Q., Walker, H.C., Steffens, P., Boehm, M., Hao, Y., QuinteroCastro, D.L., Harriger, L.W., Frontzek, M.D., Hao, L., Meng, S., Zhang, Q., Chen, G., Zhao, J., "Evidence for a Spinon Fermi Surface in a Triangular-Lattice Quantum-Spin-Liquid Candidate," Nature 540(7634), 559 (2016). 
Skripov, A.V., Isnard, O., Mushnikov, N.V., Terent'ev, P.B., Gaviko, V.S., Udovic, T.J., "Hydrogen Dynamics in the Hexagonal $\mathrm{Ho}_{2} \mathrm{Fe}_{17} \mathrm{H}_{4}$ and $\mathrm{Y}_{2} \mathrm{Fe}_{17} \mathrm{H}_{4.2}$ : Inelastic and Quasielastic Neutron Scattering Studies," J. Alloy Compd., in press. [CHRNS]

Smith, E.H., Ihlefeld, J.F., Heikes, C.A., Paik, H., Nie, Y., Adamo, C., Heeg, T., Liu, Z.-K., Schlom, D.G., "Exploiting Kinetics and Thermodynamics to Grow Phase-Pure Complex Oxides by Molecular-Beam Epitaxy under Continuous Codeposition," Phys. Rev. Mater. 1(2), 023403 (2017).

Soloninin, A.V., Dimitrievska, M., Skoryunov, R.V., Babanova, O.A., Skripov, A.V., Tang, W.S., Stavila, V., Orimo, S.-I., Udovic, T.J., "Comparison of Anion Reorientational Dynamics in $\mathrm{MCB}_{9} \mathrm{H}_{10}$ and $\mathrm{M}_{2} \mathrm{~B}_{10} \mathrm{H}_{10}(\mathrm{M}=\mathrm{Li}, \mathrm{Na})$ via Nuclear Magnetic Resonance and Quasielastic Neutron Scattering Studies," J. Phys. Chem. C 121(2), 1000 (2016)

Song, Y., Yamani, Z., Cao, C., Li, Y., Zhang, C., Chen, J.S., Huang, Q., Wu, H., Tao, J., Zhu, Y., Tian, W., Chi, S., Cao, H., Huang, Y.-B., Dantz, M., Schmitt, T., Yu, R., Nevidomskyy, A.H., Morosan, E., Si, Q., Dai, P., "A Mott Insulator Continuously Connected to Iron Pnictide Superconductors," Nat. Commun. 7, 13879 (2016).

Song, Y., Van Dyke, J., Lum, I.K., White, B.D., Jang, S., Yazici, D., Shu, L., Schneidewind, A., Čermák, P., Qiu, Y., Maple, M.B., Morr, D.K., Dai, P., "Robust Upward Dispersion of the Neutron Spin Resonance in the Heavy Fermion Superconductor $\mathrm{Ce}_{1-\mathrm{x}} \mathrm{Yb}_{\mathrm{x}} \mathrm{Coln}_{5}, "$ Nat. Phys. 7, 12774 (2016). [CHRNS]

Song, Z., Mansbach, R.A., He, H., Shih, K.-C., Baumgartner, R., Zheng, N., Ba, X., Huang, Y., Mani, D., Liu, Y., Lin, Y., Nieh, M.-P., Ferguson, A.L., Yin, L., Chen, J., "Modulation of Polypeptide Conformation through Donor-Acceptor Transformation of Side-Chain Hydrogen Bonding Ligands," Nat. Commun. 8(1), 92 (2017). [CHRNS]

Stack, A.G., Borreguero, J.M., Prisk, T.R., Mamontov, E., Wang, H.-W., Vlcek, L., Wesolowski, D.J., "Precise Determination of Water Exchanges on a Mineral Surface," Phys. Chem. Chem. Phys. 18(41), 28819 (2016)

Stalick, J.K., Waterstrat, R.M., "Crystal Structures and Phase Equilibria in the Hafnium-Palladium System," J. Phase Equilib. Diff. 37(4), 416 (2016)

Stillwell, R.L., Lin, I.-L., Harrison, N., Jaime, M., Jeffries, J.R., Butch, N.P., "Tricritical Point of the f-Electron Antiferromagnet $\mathrm{USb}_{2}$ Driven by High Magnetic Fields," Phys. Rev. B 95(1), 014414 (2017).
Stock, C., Rodriguez, E.E., Bourges, P., Ewings, R.A., Cao, H., Chi, S., Rodriguez-Rivera, J.A., Green, M.A., "Competing Spin Density Wave, Collinear, and Helical Magnetism in $\mathrm{Fe}_{1+x} \mathrm{Te}$ " Phys. Rev. B 95(14), 144407 (2017). [CHRNS]

Sun, K., Hu, L.-W., Newton, T., Wilson, E., Olson, A., "Estimate of Radiation Release from MIT Reactor with Un-Finned LEU Core During Maximum Hypothetical Accident," Prog. Nucl. Eng. 97, 231 (2017).

Sun, L., Akgun, B., Narayanan, S., Jiang, Z., Foster, M.D., "Surface Fluctuations of Polymer Brushes Swollen in Good Solvent Vapor," Macromolecules 49(19), 7308 (2016).

Sun, X., Guo, S., Dai, Q., Xiao, X., "Microstructure Characterization of Alkali-Glass Particle and Alkali-Glass Powder Reacted Gels with Neutron Scattering and Imaging Techniques," Mater. Charact., in press. [CHRNS]

Surmach, M.A., Portnichenko, P.Y., Park, J.T., Rodriguez-Rivera, J.A., Sun, D.L., Liu, Y., Lin, C.T., Inosov, D.S., "Impurity Effects on Spin Dynamics in Magnetic and Superconducting Iron Pnictides and Chalcogenides," Phys. Status Solidi B 254(1), 1600162 (2017). [CHRNS]

Suzuki, T., Chisnell, R., Devarakonda, A., Liu, Y.-T., Feng, W., Xiao, D., Lynn, J.W., Checkelsky, J.G., "Large Anomalous Hall Effect in a Half-Heusler Antiferromagnet," Nat. Phys. 12(12), 1119 (2016).

Svanedal, I., Andersson, F., Hedenström, E., Norgren, M., Edlund, H., Satija, S.K., Lindman, B., Rennie, A.R., "Molecular Organization of an Adsorbed Layer: A Zwitterionic, pH-Sensitive Surfactant at the Air/Water Interface," Langmuir 32(42), 10936 (2016).

Svanidze, E., Besara, T., Wang, J.K., Geiger, D., Prochaska, L., Santiago, J.M., Lynn, J.W., Paschen, S., Siegrist, T., Morosan, E., "Quantum Critical Point in the Sc-Doped Itinerant Antiferromagnet TiAu," Phys. Rev. B 95(22), 220405 (2017).

Takatsu, H., Hernandez, O., Yoshimune, W., Prestipino, C., Yamamoto, T., Tassel, C., Kobayashi, Y., Batuk, D., Shibata, Y., Abakumov, A.M., Brown, C.M., Kageyama, H., "Cubic Lead Perovskite $\mathrm{PbMoO}_{3}$ with Anomalous Metallic Behavior," Phys. Rev. B 95(15), 155105 (2017).

Talik, E., Lipińska, L., Guzik, A., Zajdel, P., Michalska, M., Szubka, M., Kądziołka-Gaweł, M., Paul, R.L., "Formation of Fe and Ni Substituted $\mathrm{LiMn}_{2-\mathrm{x}} \mathrm{M}_{\mathrm{x}} \mathrm{O}_{4}$ Nanopowders and their Crystal and Electronic Structure and Magnetic Properties," Mater. Sci.-Poland 35(1), 159 (2017). 
Taloni, A., Flomenbom, O., Castañeda-Priego, R., Marchesoni, F., "Single File Dynamics in Soft Materials," Soft Matter 13(6), 1096 (2017).

Tam, D.W., Song, Y., Man, H., Cheung, S.C., Yin, Z., Lu, X., Wang, W., Frandsen, B.A., Liu, L., Gong, Z., Ito, T.U., Cai, Y., Wilson, M.N., Guo, S., Koshiishi, K., Tian, W., Hitti, B., Ivanov, A., Zhao, Y., Lynn, J.W., Luke, G.M., Berlijn, T., Maier, T.A., Uemura, Y.J., Dai, P., "Uniaxial Pressure Effect on the Magnetic Ordered Moment and Transition Temperatures in $\mathrm{BaFe}_{2-x} \mathrm{~T}_{x} \mathrm{As}_{2}$ (T=Co,Ni)," Phys. Rev. B 95(6), 060505 (2017).

Tang, W.S., Yoshida, K., Soloninin, A.V., Skoryunov, R.V., Babanova, O.A., Skripov, A.V., Dimitrievska, M., Stavila, V., Orimo, S.-I., Udovic, T.J., "Stabilizing Superionic-Conducting Structures via Mixed-Anion Solid Solutions of MonocarbaCloso-Borate Salts," ACS Energy Lett. 1(4), 659 (2016). [CHRNS]

Tang, W.S., Dimitrievska, M., Chotard, J.-N., Zhou, W., Janot, R., Skripov, A.V., Udovic, T.J., "Structural and Dynamical Trends in Alkali-Metal Silanides Characterized by Neutron-Scattering Methods," J. Phys. Chem. C 120(38), 21218 (2016). [CHRNS]

Tassel, C., Goto, Y., Watabe, D., Tang, Y., Lu, H., Kuno, Y., Takeiri, F., Yamamoto, T., Brown, C.M., Hester, J., Kobayashi, Y., Kageyama, H., "High-Pressure Synthesis of Manganese Oxyhydride with Partial Anion Order," Angew. Chem. Int. Edit. 55, 9667 (2016).

Taylor, M.K., Runčevski, T., Oktawiec, J., Gonzalez, M.I., Siegelman, R.L., Mason, J.A., Ye, J., Brown, C.M., Long, J.R., "Tuning the Adsorption-Induced Phase Change in the Flexible Metal-Organic Framework Co(bdp)," J. Am. Chem. Soc. 138(45), 15019 (2016)

Toulouse, J., Iolin, E., Hennion, B., Petitgrand, D., Erwin, R., "Resonance Damping of the Terahertz-Frequency Transverse Acoustic Phonon in the Relaxor Ferroelectric $\mathrm{KTa}_{1-\mathrm{x}} \mathrm{Nb}_{\mathrm{x}} \mathrm{O}_{3}$," Phys. Rev. B 94(21), 214116 (2016).

Trinh, V., Xu, J., "An Experimental Study on Flow and Heat Transfer Characteristics of Ethanol/Polyalphaolefin Nanoemulsion Flowing through Circular Minichannels," Nanoscale Res. Lett., in press.

Tsou, A.H., López-Barrón, C.R., Jiang, P., Crowther, D.J., Zeng, Y., "Bimodal Poly(Ethylene-cb-Propylene) Comb Block Copolymers from Serial Reactors: Synthesis and Applications as Processability Additives and Blend Compatibilizers," Polymer 104, 72 (2016). [CHRNS]
Tulchinsky, Y., Hendon, C.H., Lomachenko, K.A., Borfecchia, E., Melot, B.C., Hudson, M.R., Tarver, J.D., Korzyński, M.D., Stubbs, A.W., Kagan, J.J., Lamberti, C., Brown, C.M., Dincă, M., "Reversible Capture and Release of $\mathrm{Cl}_{2}$ and $\mathrm{Br}_{2}$ with a RedoxActive Metal-Organic Framework," J. Am. Chem. Soc. 139(16), 5992 (2017).

Turkoglu, D., Downing, R.G., Chen, W., Şahin, D., Cook, J., "A ${ }^{3} \mathrm{He}$ Beam Stop for Minimizing Gamma-Ray and Fast-Neutron Background," J. Radioanal. Nucl. Ch. 311(2), 1243 (2017).

Tyagi, M., Casalini, R., Roland, C.M., "Short Time and Structural Dynamics in Polypropylene Glycol Nanocomposite," Rubber Chem. Technol. 90(2), 264 (2017). [CHRNS]

Urosev, I., Bakaic, E., Alsop, R.J., Rheinstädter, M.C., Hoare, T., "Tuning the Properties of Injectable Poly(Oligoethylene Glycol Methacrylate) Hydrogels by Controlling Precursor Polymer Molecular Weight," J. Mater. Chem. B 4(40), 6541 (2016). [CHRNS]

Van Every, E., Deyhim, A., Kulesza, J., "New Very Small Angle Neutron Scattering (VSANS) Instrument," J. Phys.: Conf. Ser. 746(1), 012025 (2016). [CHRNS]

Varley, J.B., Kweon, K., Mehta, P., Shea, P., Heo, T.W., Udovic, T.J., Stavila, V., Wood, B.C., "Understanding Ionic Conductivity Trends in Polyborane Solid Electrolytes from Ab Initio Molecular Dynamics," ACS Energy Lett. 2(1), 250 (2016).

Vetter, E.P., Geng, L., Ghatwai, P., Gilbert, D.A., Jin, Y., Soffa, W.A., Floro, J.A., "Lengthscale Effects on Exchange Coupling in Co-Pt $\mathrm{L1}_{0}+\mathrm{L1} 1_{2}$ Nanochessboards," APL Mater. 4(9), 096103 (2016).

Vlaisavljevich, B., Huck, J., Hulvey, Z., Lee, K., Mason, J.A., Neaton, J.B., Long, J.R., Brown, C.M., Alfè, D., Michaelides, A., Smit, B., "Performance of van der Waals Corrected Functionals for Guest Adsorption in the $\mathrm{M}_{2}$ (dobdc) Metal-Organic Frameworks," J. Phys. Chem. A 121(21), 4139 (2017).

Vu, T., Tran, T.N., Xu, J., "Single-Phase Flow and Heat Transfer Characteristics of Ethanol/Polyalphaolefin Nanoemulsion Fluids in Circular Minichannels," Int. J. Mass Tran., in press.

Wachsman, E., Lundgren, C., Takeuchi, E., Xu, K., Wang, C., Dura, J., Jacobson, D., Amine, K., Acker, W., "The Center for Research on Extreme Batteries," Electrochem. Soc. Interface 25(3), 26 (2016).

Wah, B., Breidigan, J.M., Adams, J., Horbal, P., Garg, S., Porcar, L., Perez-Salas, U., "Reconciling Differences between Lipid Transfer in Free-Standing and Solid Supported Membranes: A TimeResolved Small-Angle Neutron Scattering Study," Langmuir 33(14), 3384 (2017). [CHRNS] 
Wang, C., Wiener, C.G., Cheng, Z., Vogt, B.D., Weiss, R.A., "Modulation of the Mechanical Properties of Hydrophobically Modified Supramolecular Hydrogels by Surfactant-Driven Structural Rearrangement," Macromolecules 49(23), 9228 (2016). [CHRNS]

Wang, C., Wiener, C.G., Yang, Y., Weiss, R.A., Vogt, B.D., "Structural Rearrangement and Stiffening of Hydrophobically Modified Supramolecular Hydrdogels During Thermal Annealing," J. Polym. Sci. Pol. Phys. 55(13), 1036 (2017). [CHRNS]

Wang, G., Chu, P.-H., Fu, B., He, Z., Kleinhenz, N., Yuan, Z., Mao, Y., Wang, H., Reichmanis, E., "Conjugated Polymer Alignment: Synergisms Derived from Microfluidic Shear Design and UV Irradiation," ACS Appl. Mater. Interfaces 8(37), 24761 (2016).

Wang, H., Wu, H., Kan, J., Chang, G., Yao, Z., Li, B., Zhou, W., Xiang, S., Zhao, J.C.-G., Chen, B., "A Microporous HydrogenBonded Organic Framework with Amine Sites for Selective Recognition of Small Molecules," J. Mater. Chem. A 5(18), 8292 (2017).

Wang, J.K., Wu, S., Qiu, Y., Rodriguez-Rivera, J.A., Huang, Q., Broholm, C., Morosan, E. "Modulated Magnetism and Anomalous Electronic Transport in $\mathrm{Ce}_{3} \mathrm{Cu}_{4} \mathrm{As}_{4} \mathrm{O}_{2}$," Phys. Rev. B 94(6) 064430 (2016). [CHRNS]

Wang, Y., Wang, D., Yuan, G., Ma, H., Xu, F., Li, J., Viehland, D., Gehring, P.M., "Fragile Morphotropic Phase Boundary and Phase Stability in the Near-Surface Region of the Relaxor Ferroelectric (1-x) $\mathrm{Pb}\left(\mathrm{Zn}_{1 / 3} \mathrm{Nb}_{2 / 3}\right) \mathrm{O}_{3-x} \mathrm{PbTiO}_{3}$ : [001] Field-Cooled Phase Diagrams," Phys. Rev. B 94(17), 174103 (2016).

Wang, Z., Lam, C.N., Chen, W.-R., Wang, W., Liu, J., Liu, Y., Porcar, L., Stanley, C.B., Zhao, Z., Hong, K., Wang, Y., "Fingerprinting Molecular Relaxation in Deformed Polymers," Phys. Rev. X 7(3), 031003 (2017). [CHRNS]

Weitzhandler, I., Dzuricky, M., Hoffmann, I., Garcia Quiroz, F., Gradzielski, M., Chilkoti, A., "Micellar Self-Assembly of Recombinant Resilin-Like/Elastin-Like Block Copolypeptides," Biomacromolecules, in press. [CHRNS]

Wen, H.-M., Chang, G., Li, B., Liu, R.-B., Hu, T.-L., Zhou, W., Chen, B., "Highly Enhanced Gas Uptake and Selectivity via Incorporating Methoxy Groups into a Microporous MetalOrganic Framework," Cryst. Growth Des. 17(4), 2172 (2017).

Wen, J.-J., Koohpayeh, S.M., Ross, K.A., Trump, B.A., McQueen, T.M., Kimura, K., Nakatsuji, S., Qiu, Y., Pajerowski, D.M., Copley, J.R.D., Broholm, C.L., "Disordered Route to the Coulomb Quantum Spin Liquid: Random Transverse Fields on Spin Ice in $\mathrm{Pr}_{2} \mathrm{Zr}_{2} \mathrm{O}_{7}$," Phys. Rev. Lett. 118(10), 107206 (2017).
White, J.L., Newhouse, R.J., Zhang, J.Z., Udovic, T.J., Stavila, V., "Understanding and Mitigating the Effects of Stable Dodecahydro-Closo-Dodecaborate Intermediates on Hydrogen-Storage Reactions," J. Phys. Chem. C 120(45), 25725 (2016).

Wiener, C.G., Wang, C., Liu, Y., Weiss, R.A., Vogt, B.D., "Nanostructure Evolution During Relaxation from a Large Step Strain in a Supramolecular Copolymer-Based Hydrogel: A SANS Investigation," Macromolecules 50(4), 1672 (2017). [CHRNS]

Williams, T.J., Aczel, A.A., Stone, M.B., Wilson, M.N., Luke, G.M., "Hidden Order Signatures in the Antiferromagnetic Phase of $\mathrm{U}\left(\mathrm{Ru}_{1-x} \mathrm{Fe}_{\mathrm{x}}\right)_{2} \mathrm{Si}_{2}, "$ Phys. Rev. B 95(10), 104440 (2017). [CHRNS]

Williams, T.J., Barath, H., Yamani, Z., Rodriguez-Riviera, J.A., Leão, J.B., Garrett, J.D., Luke, G.M., Buyers, W.J.L., Broholm, C., "Gapped Excitations in the High-Pressure Antiferromagnetic Phase of URu $\mathrm{Si}_{2}$, " Phys. Rev. B 95(19), 195171 (2017). [CHRNS]

Wood, B.C., Stavila, V., Poonyayant, N., Heo, T.-W., Ray, K.G., Klebanoff, L.E., Udovic, T.J., Lee, J.R.I., Angboonpong, N., Sugar, J.D., Pakawatpanurut, P., "Nanointerface-Driven Reversible Hydrogen Storage in the Nanoconfined Li-N-H System," Adv. Mater. Interfaces 4(3), 1600803 (2017).

Worthington, P., Drake, K.M., Li, Z., Napper, A.D., Pochan, D.J., Langhans, S.A., "Beta-Hairpin Hydrogels as Scaffolds for HighThroughput Drug Discovery in Three-Dimensional Cell Culture," Anal. Biochem., in press.

Wu, H., Khaykovich, B., Wang, X., Hussey, D.S., "Wolter Mirrors for Neutron Imaging," Phys. Procedia 88, 184 (2017).

Wu, H., Tang, W.S., Zhou, W., Tarver, J.D., Stavila, V., Brown, C.M., Udovic, T.J., "The Low-Temperature Structural Behavior of Sodium 1-Carba-closo-Decaborate: $\mathrm{NaCB}_{9} \mathrm{H}_{10}$," J. Solid State Chem. 243, 162 (2016).

Wu, H., Zhou, X., Rodriguez, E.E., Zhou, W., Udovic, T.J., Yildirim, T., Rush, J.J., "A New Family of Metal Borohydride Guanidinate Complexes: Synthesis, Structures and Hydrogen-Storage Properties," J. Solid State Chem. 242(1), 186 (2016).

Wu, Z., Williams, R.E., "The Water Ingress Effects on the Reactivity Change of the Conceptual Designed Reactor at NIST," T. Am. Nucl. Soc. 115, 1186 (2016).

Wu, Z., Williams, R.E., Rowe, J.M., Newton, T.H., O'Kelly, S., "Neutronics and Safety Studies on a Research Reactor Concept for an Advanced Neutron Sources," Nucl. Technol. 199, 67 (2017). 
Xi, Y., Pozzo, L.D., "Electric Field Directed Formation of Aligned Conjugated Polymer Fibers," Soft Matter 13(21), 3894 (2017).

Xia, Y., Charubin, K., Marquardt, D., Heberle, F.A., Katsaras, J., Tian, J., Cheng, X., Liu, Y., Nieh, M.-P., "Morphology-Induced Defects Enhance Lipid Transfer Rates," Langmuir 32(38), 9757 (2016).

Xuan, S., Gupta, S., Li, X., Bleuel, M., Schneider, G.J., Zhang, D., "Synthesis and Characterization of Well-Defined PEGylated Polypeptoids as Protein-Resistant Polymers," Biomacromolecules 18(3), 951 (2017)

Yajima, T., Koshiko, M., Zhang, Y., Oguchi, T., Yu, W., Kato, D., Kobayashi, Y., Orikasa, Y., Yamamoto, T., Uchimoto, Y., Green, M.A., Kageyama, H., "Selective and Low Temperature Transition Metal Intercalation in Layered Tellurides," Nat. Commun. 7, 13809 (2016).

Yamaguchi, T., Faraone, A., "Analysis of Shear Viscosity and Viscoelastic Relaxation of Liquid Methanol Based on Molecular Dynamics Simulation and Mode-Coupling Theory," J. Chem. Phys. 146(24), 244506 (2017). [CHRNS]

Yamamuro, O., Kofu, M., "Calorimetric and Neutron Scattering Studies on Glass Transitions and Ionic Diffusions in ImidazoliumBased Ionic Liquids," IOP Conf. Ser.-Mat. Sci. 196(1), 012001 (2016). [CHRNS]

Yang, J., Samarakoon, A.M., Hong, K.W., Copley, J.R.D., Huang, Q., Tennant, A., Sato, T.J., Lee, S.-H., "Glassy Behavior and Isolated Spin Dimers in a New Frustrated Magnet $\mathrm{BaCr}_{9 \mathrm{p}} \mathrm{Ga}_{12-9} \mathrm{pO}_{19}$," J. Phys. Soc. Jpn. 85(9), 094712 (2016).

Yang, K., Cai, Z., Jaiswal, A., Tyagi, M., Moore, J.S., Zhang, Y., "Dynamic Odd-Even Effect in Liquid $n$-Alkanes Near their Melting Points," Angew. Chem. Int. Edit. 55(45), 14090 (2016).

Yang, W., Yang, F., Hu, T.-L., King, S.C., Wang, H., Wu, H., Zhou, W., Li, J.-R., Arman, H.D., Chen, B., "Microporous DiaminotriazineDecorated Porphyrin-Based Hydrogen-Bonded Organic Framework: Permanent Porosity and Proton Conduction," Cryst. Growth Des. 16(10), 5831 (2016).

Yin, C., Tian, G., Li, G., Liao, F., Lin, J., "New 10H Perovskites $\mathrm{Ba}_{5} \mathrm{Ln}_{1-\mathrm{x}} \mathrm{Mn}_{4+\mathrm{y}} \mathrm{O}_{15-\delta}$ with Spin Glass Behaviour," RSC Adv. 7(54), 33869 (2017).

Yoshida, K., Sato, T., Unemoto, A., Matsuo, M., Ikeshoji, T., Udovic, T.J., Orimo, S.-I., "Fast Sodium Ionic Conduction in $\mathrm{Na}_{2} \mathrm{~B}_{10} \mathrm{H}_{10}-\mathrm{Na}_{2} \mathrm{~B}_{12} \mathrm{H}_{12}$ Pseudo-Binary Complex Hydride and Application to a Bulk-Type All-Solid-State Battery," Appl. Phys. Lett. 110(10), 103901 (2017).
Yu, J., Mao, J., Yuan, G., Satija, S., Jiang, Z., Chen, W., Tirrell, M., "Structure of Polyelectrolyte Brushes in the Presence of Multivalent Counterions," Macromolecules 49(15), 5609 (2016).

Yu, J., Mao, J., Yuan, G., Satija, S., Chen, W., Tirrell, M., "The Effect of Multivalent Counterions to the Structure of Highly Dense Polystyrene Sulfonate Brushes," Polymer 98, 448 (2016).

Yu, L., Yan, Z., Yang, H.-C., Chai, X., Li, B., Moeendarbari, S., Hao, Y., Zhang, D., Feng, G., Han, P., Gilbert, D.A., Liu, K., Buchanan, K.S., Cheng, X., "Magnetization Reversal of ThreeDimensional Nickel Anti-Sphere Arrays," IEEE Magn. Lett. 8, 4100104 (2016)

Yu, L.L., Stanoyevitch, R.C., Zeisler, R., "SI Traceable Determination of Arsenic Species in Kelp (Thallus Laminariae)," Anal. Methods-UK, in press.

Yuan, G., Cheng, H., Han, C.C., "The Glass Formation of a Repulsive System with Also a Short Range Attractive Potential: A Re-Interpretation of the Free Volume Theory," Polymer, in press. [CHRNS]

Yuan, G., Wang, X., Wu, D., Hammouda, B., "Structural Analysis of Dendrimers Based on Polyhedral Oligomeric Silsesquioxane and their Assemblies in Solution by Small-Angle Neutron Scattering: Fits to a Modified Two Correlation Lengths Model," Polymer 100, 119 (2016). [CHRNS]

Yuan, G., Luo, J., Han, C.C., Liu, Y., "Gelation Transitions of Colloidal Systems with Bridging Attractions," Phys. Rev. E 94(4), 040601 (R) (2016). [CHRNS]

Zabet, M., Mishra, S., Boy, R., Walters, K.B., Naskar, A.K., Kundu, S., "Temperature-Dependent Self-Assembly and Rheological Behavior of a Thermoreversible PMMA-Pn BA-PMMA Triblock Copolymer Gel," J. Polym. Sci. Pol. Phys. 55(11), 877 (2017). [CHRNS]

Zajdel, P., Li, W.-Y., van Beek, W., Lappas, A., Ziolkowska, A., Jaskiewicz, S., Stock, C., Green, M.A., "Structure and Magnetism in the Bond-Frustrated Spinel $\mathrm{ZnCr}_{2} \mathrm{Se}_{4}$," Phys. Rev. B 95(13), 134401 (2017).

Zajdel, P., Gagor, A., Pajerowski, D.M., Ptake, M., Szlawski, M., "Suppression of the Commensurate Magnetic Phase in Nanosized Hübnerite $\mathrm{MnWO}_{4}$," Phys. Rev. B 95(17), 174427 (2017).

Zeisler, R., Souza Ribeiro, Jr., I., Shetty, M.G., Turkoglu, D., "On Neutron Activation Analysis with $\gamma \gamma$ Coincidence Spectrometry," J. Radioanal. Nucl. Ch., in press. 
Zhang, H.V., Polzer, F., Haider, M.J., Tian, Y., Villegas, J.A., Kiick, K.L., Pochan, D.J., Saven, J.G., "Computationally Designed Peptides for Self-Assembly of Nanostructured Lattices," Sci. Adv. 2(9), e1600307 (2016). [CHRNS]

Zhang, M., Zhou, W., Pham, T., Forrest, K.A., Liu, W., He, Y., Wu, H., Yildirim, T., Chen, B., Space, B., Pan, Y., Zaworotko, M.J., Bai, J., "Fine Tuning of MOF-505 Analogues to Reduce Low Pressure Methane Uptake and Enhance Methane Working Capacity," Angew. Chem. Int. Edit., in press.

Zhang, M., Chen, C., Wang, Q., Fu, W., Huang, K., Zhou, W., "A MetalOrganic Framework Functionalized with Piperazine Exhibiting Enhanced $\mathrm{CH}_{4}$ Storage," J. Mater. Chem. A 5(1), 349 (2016).

Zhang, W., Howell, S.C., Wright, D.W., Heindel, A., Qiu, X., Chen, J., Curtis, J.E., "Combined Monte Carlo/Torsion-Angle Molecular Dynamics for Ensemble Modeling of Proteins, Nucleic Acids, and Carbohydrates," J. Mol. Graph. Model. 73, 179 (2017).

Zhang, W., Shen, H., Guralnick, B.W., Kirby, B.J., Nguyen, N.A., Remy, R., Majkrzak, C.F., Mackay, M.E., "Correlation between Morphology and Device Performance of pBTTT:PC ${ }_{71}$ BM Solar Cells," Sol. Energ. Mat. Sol. C. 155, 387 (2016). [CHRNS]

Zhang, W., Ngyuen, N.A., Murray, R., Mackay, M.E., “Device Performance Enhancement of Polymer Solar Cells by Nanoparticle Self-Assembly," Sol. Energ. Mat. Sol. C. 160, 126 (2017). [CHRNS]

Zhang, Y., Zhao, W., Yang, J., Hammouda, B., Yang, J., Cheng, G., "SANS Study on Self-Assembled Structures of Glucose-Responsive Phenylboronate Ester-Containing Diblock Copolymer," Eur. Polym. J. 83, 173 (2016). [CHRNS]

Zhang, Z., Liu, Y., "Recent Progresses of Understanding the Viscosity of Concentrated Protein Solutions," Curr. Opin. Chem. Eng. 16, 48 (2017).

Zhao, D., Gimenez-Pinto, V., Jimenez, A.M., Zhao, L., Jestin, J., Kumar, S.K., Kuei, B., Gomez, E.D., Prasad, A.S., Schadler, L.S., Khani, M.M., Benicewicz, B.C., "Tunable Multiscale Nanoparticle Ordering by Polymer Crystallization," ACS Cent. Sci. 3(7), 751 (2017). [CHRNS]
Zhao, E., Fang, L., Chen, M., Chen, D., Huang, Q., Hu, Z., Yan, Q.-B., Wu, M., Xiao, X., "New Insight into Li/Ni Disorder in Layered Cathode Materials for Lithium Ion Batteries: A Joint Study of Neutron Diffraction, Electrochemical Kinetic Analysis and FirstPrinciples Calculations," J. Mater. Chem. A 5(4), 1679 (2017).

Zhao, W., Xiao, L.-P., Song, G., Sun, R.-C., He, L., Singh, S., Simmons, B.A., Cheng, G., "From Lignin Subunits to Aggregates: Insights into Lignin Solubilization," Green Chem. 19(14), 3272 (2017). [CHRNS]

Zhao, Y., Lynn, J.W., Thakur, G.S., Haque, Z., Gupta, L.C., Ganguli, A.K., "Magnetic Structures of Rare Earth Intermetallic Compounds $\mathrm{RCuAs}_{2}(\mathrm{R}=\mathrm{Pr}$, Nd, Tb, Dy, Ho, and Yb)," J. Phys. Chem. Solids, in press.

Zheng, X.Q., Zhang, B., Wu, H., Hu, F.X., Huang, Q.Z., Shen, B.G., "Large Magnetocaloric Effect of $\mathrm{Ho}_{x} \mathrm{Er}_{1-x} \mathrm{Ni}(0 \leq \mathrm{x} \leq 1)$ Compounds," J. Appl. Phys. 120(16), 163907 (2016).

Zheng, X., Zhang, B., Li, Y., Wu, H., Zhang, H., Zhang, J., Wang, S., Huang, Q., Shen, B., "Large Magnetocaloric Effect in $\mathrm{Er}_{12} \mathrm{CO}_{7}$ Compound and the Enhancement of $\delta T_{\mathrm{FWHM}}$ by Ho-Substitution," J. Alloy Compd. 680, 617 (2016).

Zhou, X., Wilfong, B., Vivanco, H., Paglione, J., Brown, C.M., Rodriguez, E.E., "Metastable Layered Cobalt Chalcogenides from Topochemical Deintercalation," J. Am. Chem. Soc. 138(50), 16432 (2016).

Zhou, X., Zhou, W., Udovic, T.J., Yildirim, T., Rush, J.J., Rodriguez, E.E., Wu, H., "Development of Potential OrganicMolecule-Based Hydrogen Storage Materials: Converting C-N Bond-Breaking Thermolysis of Guanidine to $\mathrm{N}-\mathrm{H}$ BondBreaking Dehydrogenation," Int. J. Hydrogen Energ. 41(41), 18542 (2016).

Zhukhovitskiy, A.V., Zhao, J., Zhong, M., Keeler, E.G., Alt, E.A., Teichen, P., Griffin, R.G., Hore, M.J.A., Willard, A.P., Johnson, J.A., "Polymer Structure Dependent Hierarchy in PolyMOC Gels," Macromolecules 49(18), 6896 (2016). 


\section{Instruments and Contacts}

(name, tel. 301-975-xxxx, email)

High resolution powder diffractometer (BT-1):

- J. K. Stalick, 6223, judith.stalick@nist.gov

- H. Wu, 2387, hui.wu@nist.gov

- Q. Z. Huang, 6164, qing.huang@nist.gov

- C. M. Brown, 5134, craig.brown@nist.gov

Residual stress diffractometer (BT-8):

- T. Gnäupel-Herold, 5380, thomas.gnaeupel-herold@nist.gov

30-m SANS instrument (NG-7):

- Y. Liu, 6235, yun.liu@nist.gov

- S. Teixeira, 4404 susana.marujoteixeira@nist.gov

- Y. Mao, 5250, yimin.mao@nist.gov

- J. R. Krzywon, 6650, jkrzywon@nist.gov

30-m SANS instrument (NG-B 30m) (CHRNS):

- B. Hammouda, 3961, hammouda@nist.gov

- P. D. Butler, 2028, paul.butler@nist.gov

- S. Krueger, 6734, susan.krueger@nist.gov

- C. Gagnon, 2020, cedric.gagnon@nist.gov

10-m SANS instrument (NG-B) (nSoft):

- R. Jones, 4624, ronald.jones@nist.gov

- K. Weigandt, 8396, kathleen.weigandt@nist.gov

USANS, Perfect Crystal SANS (BT-5) (CHRNS):

- M. Bleuel, 5165, markus.bleuel@nist.gov

- P. D. Butler, 2028, paul.butler@nist.gov

vSANS instrument (NG-3) (CHRNS):

- G. Jensen, 4591, grethe.jensen@nist.gov

- J. G. Barker, 6732, john.barker@nist.gov

- E. Kelley, 8584, elizabeth.kelley@nist.gov

- C. Gagnon, 2020, cedric.gagnon@nist.gov

- C. J. Glinka, 6242, charles.glinka@nist.gov

Polarized Beam Reflectometer/Diffractometer (NG-D):

- B. J. Kirby, 8395, brian.kirby@nist.gov

- J. A. Borchers, 6597, julie.borchers@nist.gov

- C. F. Majkrzak, 5251, cmajkrzak@nist.gov

MAGIK, Off-Specular Reflectometer (NG-D):

- B. B. Maranville, 6034, brian.maranville@nist.gov

- J. A. Dura, 6251, joseph.dura@nist.gov

Neutron reflectometer-horizontal sample (NG-7):

- S. K. Satija, 5250, satija@nist.gov
Double-focusing triple-axis spectrometer (BT-7):

- Y.Zhao, 2164, yang.zhao@nist.gov

- D. Parshall, 8097, daniel.parshall@nist.gov

- J.W. Lynn, 6246, jeff.lynn@nist.gov

SPINS, Spin-polarized triple-axis spectrometer (NG-5):

- G. Xu, 4144, guangyong.xu@nist.gov

- L. Harriger, 8360, leland.harriger@nist.gov

Triple-axis spectrometer (BT-4):

-W. Ratcliff, 4316, william.ratcliff@nist.gov

FANS, Filter-analyzer neutron spectrometer (BT-4):

- T. J. Udovic, 6241, udovic@nist.gov

DCS, Disk-chopper time-of-flight spectrometer (NG-4):

- N. Butch, 4863, nicholas.butch@nist.gov

- W. Zhou, 8169, wei.zhou@nist.gov

- C. M. Brown, 5134, craig.brown@nist.gov

HFBS, High-flux backscattering spectrometer (NG-2) (CHRNS):

- T. Prisk, 5010, timothy.prisk@nist.gov

- M. Tyagi, 2046, madhusudan.tyagi@nist.gov

NSE, Neutron spin echo spectrometer (NG-A) (CHRNS):

- A. Faraone, 5254, antonio.faraone@nist.gov

- M. Nagao, 5505, michihiro.nagao@nist.gov

MACS, Multi-angle crystal spectrometer (BT-9) (CHRNS):

- J. A. Rodriguez-Rivera,6019, jose.rodriguez@nist.gov

- Y. Qiu, 3274, yiming.qiu@nist.gov

Cold-neutron prompt-gamma neutron activation analysis (NG-D):

- H. H. Chen-Mayer, 5595, heather.chen-mayer@nist.gov

- R. L. Paul, 6287, rpaul@nist.gov

Thermal-neutron prompt-gamma activation analysis (VT-5):

- R. L. Paul, 6287, rpaul@nist.gov

Other activation analysis facilities:

- N. Sharp, 3926, nicholas.sharp@nist.gov

- P. Chu, 2988, pamela.chu@nist.gov

Cold neutron depth profiling (NG-1):

- H. H. Chen-Mayer, 5595, heather.chen-mayer@nist.gov

- J. Weaver, 6311, jamie.weaver@nist.gov 
Thermal Neutron Imaging Station (BT-2):

- D. Jacobson, 6207, david.jacobson@nist.gov

- D. Hussey, 6465, daniel.hussey@nist.gov

- E. Baltic, 4842, eli.baltic@nist.gov

- J. Lamanna, 6809, jacob.lamanna@nist.gov

- M. Arif, 6303, muhammad.arif@nist.gov

Cold Neutron Imaging Station (NG-6):

- D. Jacobson, 6207, david.jacobson@nist.gov

- D. Hussey, 6465, daniel.hussey@nist.gov

- E. Baltic, 4842, eli.baltic@nist.gov

- J. Lamanna, 6809, jacob.lamanna@nist.gov

- M. Arif, 6303, muhammad.arif@nist.gov

Neutron interferometer (NG-7):

- M. Huber, 5641, michael.huber@nist.gov

- D. Pushin, 4792, dmitry.pushin@nist.gov

- M. Arif, 6303, muhammad.arif@nist.gov

Quantum-based neutron interferometer facility (NG-7):

- M. Huber, 5641, michael.huber@nist.gov

- D. Pushin, 4792, dmitry.pushin@nist.gov

- M. Arif, 6303, muhammad.arif@nist.gov

Fundamental neutron physics station (NG-C):

- S. Hoogerheide, 8582, shannon.hoogerheide@nist.gov

- M. S. Dewey, 4843, maynard.dewey@nist.gov

- J. Nico, 4663, nico@nist.gov
Fundamental neutron physics station (NG-6):

- NG-6M: M. S. Dewey, 4843, mdewey@nist.gov

- NG-6M: H. Mumm, 8355, hans.mumm@nist.gov

- NG-6A (MDM): M. Huber, 5641, michael.huber@nist.gov

- NG-6U: H. Mumm, 8355, hans.mumm@nist.gov

- NG-6U: A. Thompson, 4666, alan.thompson@nist.gov

Neutron test station (NG-7):

- R. Erwin, 6245, ross.erwin@nist.gov

- K. Krycka, 8685, kathryn.krycka@nist.gov

Theory and modeling:

- J. E. Curtis, 3959, joseph.curtis@nist.gov

- T. Yildirim, 6228, taner@nist.gov

\section{Instruments under development:}

CANDOR, White-beam reflectometer/diffractometer:

- A. Grutter, 4198, alexander.grutter@nist.gov

- D. Hoogerheide, 8839, david.hoogerheide@nist.gov

- F. Heinrich, 4507, frank.heinrich@nist.gov

- C. F. Majkrzak, 5251, charles.majkrzak@nist.gov 


\section{NIST Center for Neutron Research Contacts}

Copies of annual reports, facility information, user information, and research proposal guidelines are available electronically.

Please visit our website: http://www.ncnr.nist.gov

\section{For a paper copy of this report:}

Steve Kline

301-975-6243

steven.kline@nist.gov

\section{For general information on the facility:}

Rob Dimeo

301-975-6210

robert.dimeo@nist.gov

Dan Neumann

301-975-5252

dan.neumann@nist.gov

\author{
For information on visiting the \\ facility and/or user access questions: \\ Julie Keyser \\ 301-975-8200 \\ julie.keyser@nist.gov \\ Mary Ann FitzGerald \\ 301-975-8200 \\ maryann.fitzgerald@nist.gov \\ Becky Ogg \\ 301-975-8200 \\ rebecca.ogg@nist.gov

\section{For information on performing} \\ research at the facility: \\ Yamali Hernandez \\ 301-975-5295 \\ yamali.hernandez@nist.gov

\section{Facility address:} \\ NIST Center for Neutron Research \\ National Institute of Standards and Technology \\ 100 Bureau Drive, Mail Stop 6100 \\ Gaithersburg, MD 20899-6100 USA
}


This publication is available free of charge from: https://doi.org/10.6028/NIST.SP.1216 


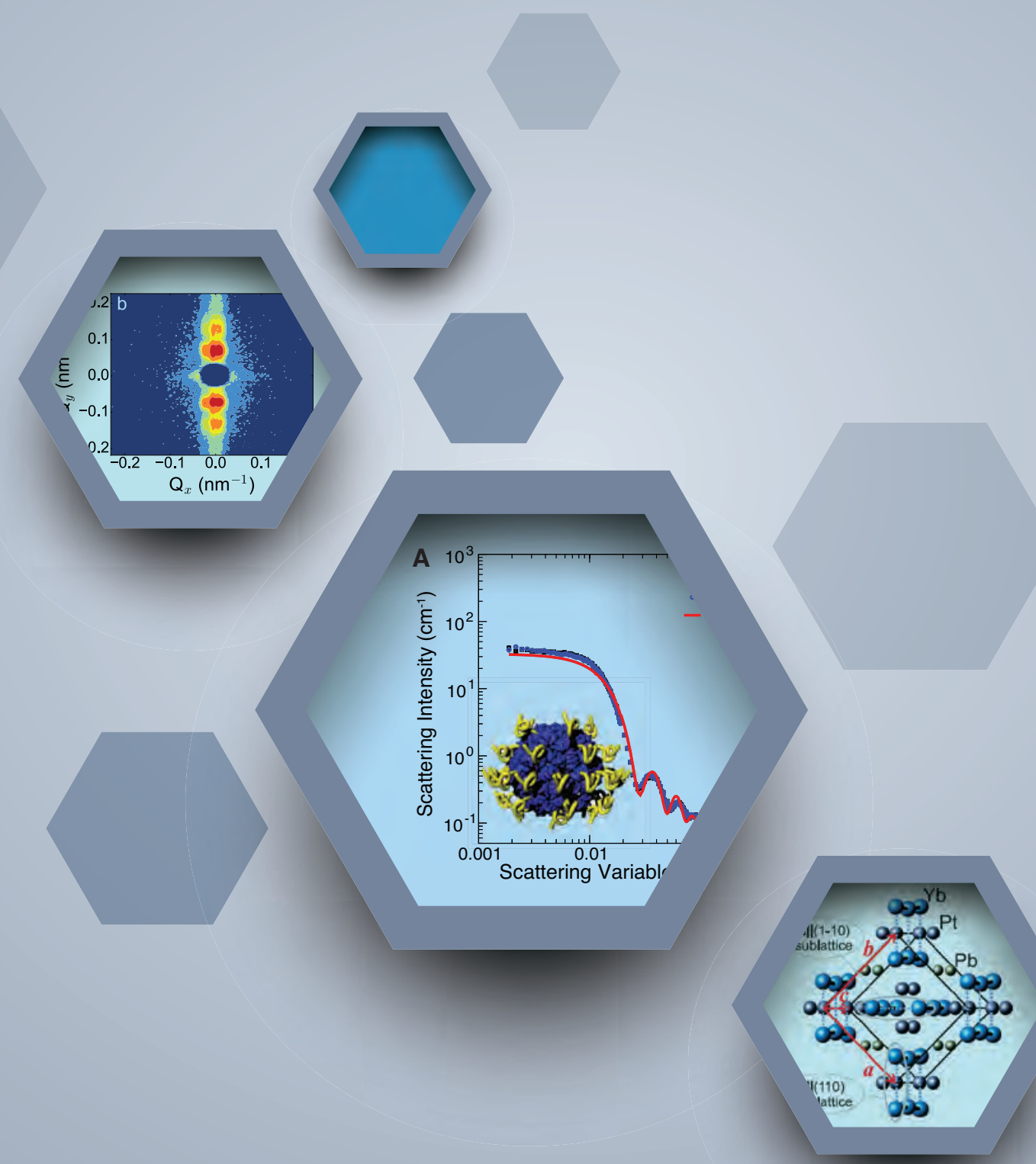

NIST CENTER FOR NEUTRON RESEARCH National Institute of Standards and Technology 100 Bureau Drive, MS 6100

Gaithersburg, MD 20899-6100

www.ncnr.nist.gov 